\title{
Extra-articular manifestations and comorbidities in spondyloarthritis : epidemiological and clinical aspects
}

Citation for published version (APA):

Stolwijk, C. (2015). Extra-articular manifestations and comorbidities in spondyloarthritis : epidemiological and clinical aspects. [Doctoral Thesis, Maastricht University]. Maastricht University. https://doi.org/10.26481/dis.20150424cs

Document status and date:

Published: 01/01/2015

DOI:

$10.26481 /$ dis.20150424cs

Document Version:

Publisher's PDF, also known as Version of record

Please check the document version of this publication:

- A submitted manuscript is the version of the article upon submission and before peer-review. There can be important differences between the submitted version and the official published version of record.

People interested in the research are advised to contact the author for the final version of the publication, or visit the DOI to the publisher's website.

- The final author version and the galley proof are versions of the publication after peer review.

- The final published version features the final layout of the paper including the volume, issue and page numbers.

Link to publication

\footnotetext{
General rights rights.

- You may freely distribute the URL identifying the publication in the public portal. please follow below link for the End User Agreement:

www.umlib.nl/taverne-license

Take down policy

If you believe that this document breaches copyright please contact us at:

repository@maastrichtuniversity.nl

providing details and we will investigate your claim.
}

Copyright and moral rights for the publications made accessible in the public portal are retained by the authors and/or other copyright owners and it is a condition of accessing publications that users recognise and abide by the legal requirements associated with these

- Users may download and print one copy of any publication from the public portal for the purpose of private study or research.

- You may not further distribute the material or use it for any profit-making activity or commercial gain

If the publication is distributed under the terms of Article $25 \mathrm{fa}$ of the Dutch Copyright Act, indicated by the "Taverne" license above, 
Extra-articular manifestations and comorbidities in spondyloarthritis: epidemiological and clinical aspects

Carmen Stolwijk 2015 
ISBN 978-94-6169-645-8

Printing was financially supported by Abbvie, Pfizer B.V., UCB Pharma B.V., Dutch Arthritis Foundation

Cover design: Jozien Stolwijk-Kooter

Layout and printing: Optima Grafische Communicatie, Rotterdam, The Netherlands 
Extra-articular manifestations and comorbidities in spondyloarthritis: epidemiological and clinical aspects

\author{
PROEFSCHRIFT
}

Ter verkrijging van de graad van doctor aan de Universiteit van Maastricht, op gezag van de Rector Magnificus, Prof. Dr. L.L.G. Soete volgens het besluit van het College van Decanen, in het openbaar te verdedigen op vrijdag 24 april 2015 om 14.00

Door

Carmen Stolwijk 
Promotor:

Prof. dr. A.E.R.C.H. Boonen

Copromotor:

Dr. A.M. van Tubergen

Leden van de beoordelingscommissie:

Prof. dr. N.C. Schaper

Prof. dr. M.H. Prins

Prof. dr. M. Boers

Dr. F. van Gaalen

Dr. M. van den Akker 


\section{CONTENT}

Chapter 1 General introduction

Part I Epidemiology of spondyloarthritis

Chapter 2 The global prevalence of spondyloarthritis: a systematic review and meta-regression analysis

\section{Part II Extra-articular manifestations}

Chapter 3 Prevalence of extra-articular manifestations in patients with ankylosing spondylitis: a systematic review and meta-analysis

Chapter 4 Ankylosing spondylitis and the epidemiology of extra-articular manifestations: a population-based matched cohort study

Chapter 5 Prevalence of self-reported spondyloarthritis features in a cohort of patients with inflammatory bowel disease

\section{Part III Comorbidities in ankylosing spondylitis}

Chapter 6 Aspects of validity of the self-administered comorbidity questionnaire (SCO) in patients with ankylosing spondylitis

Chapter 7 Ankylosing spondylitis and risk of ischaemic heart disease: a population-based cohort study

Chapter 8 Infliximab treatment reduces depressive symptoms in patients with ankylosing spondylitis: a subgroup analysis of a randomized, placebo-controlled trial (ASSERT)

Chapter 9 General discussion

Chapter 10 Summary

Chapter 11 Samenvatting in het Nederlands

Valorisation addendum

Dankwoord

Curriculum Vitae

Publications 



\section{CHAPTER 1 General introduction}

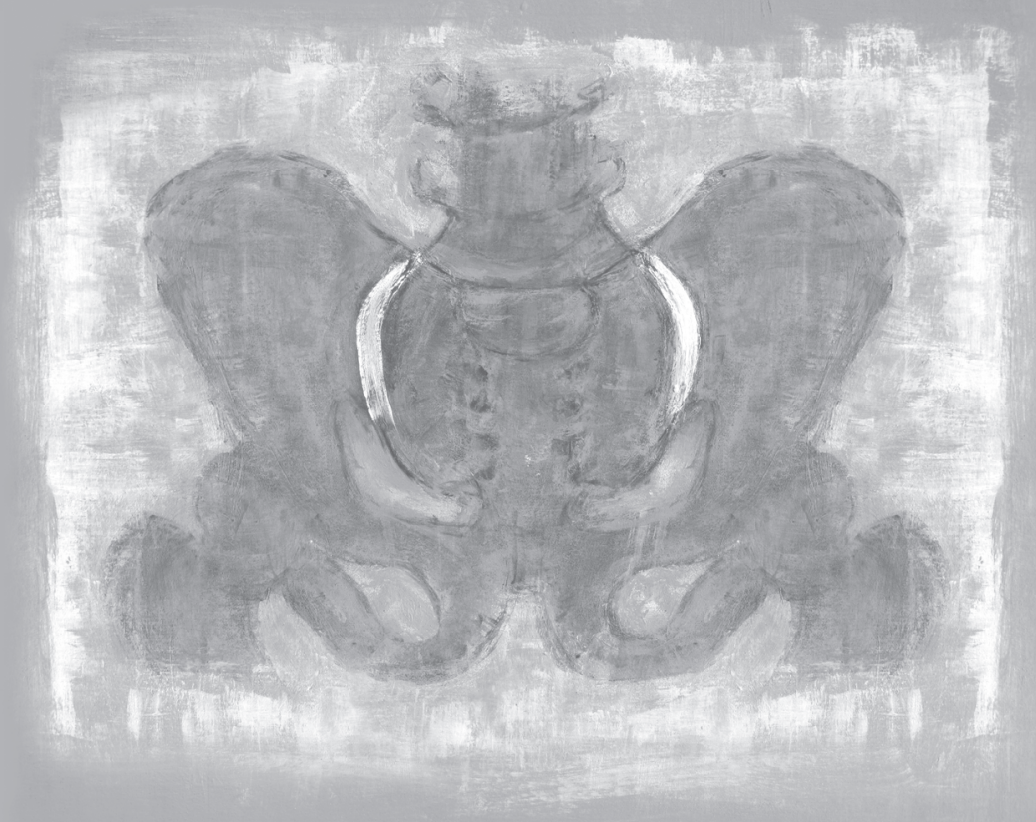





\section{GENERAL INTRODUCTION}

During the 1960s, Moll and Wright observed in family studies a striking association between ankylosing spondylitis (AS) and several other disorders, such as psoriatic arthritis (PsA) and arthritis related to Crohn's disease or ulcerative colitis. In 1974, a global concept overarching these disorders was established and a collective name was chosen: seronegative spondyloarthropathies [1]. The addition of the term 'seronegative' referred to the absence of rheumatoid factors. The discovery of the genetic association of the diseases belonging to the proposed concept with the MHC class I molecule human leucocyte antigen (HLA) B27 shortly thereafter, further legitimized the 'spondyloarthopathies' [2]. To better reflect the inflammatory character of the diseases, the term spondyloarthropathies was later replaced by spondyloarthritis (SpA) [3]. The disorders that are part of the SpA concept share, although in varying prevalence, several clinical features, including inflammation of the axial skeleton (spine and sacroiliac joints), peripheral joints (especially the large joints of the lower extremities) or entheses, but also extra-articular manifestations (EAMs) of which the most striking are acute anterior uveitis (AAU), psoriasis and inflammatory bowel disease (IBD). Clearly, all possible combinations of the different manifestations can result in a wide range of phenotypes. In practice, a limited number of phenotypic subforms of SpA are distinguished, including AS, PsA, SpA related to IBD, reactive arthritis (ReA) and undifferentiated SpA (uSpA). This subdivision is mainly based on the presence of sacroiliitis on x-ray (AS), and the presence of EAMs (psoriasis or IBD) or the pathogenesis (ReA). Patients with USpA have symptoms of SpA, but lack other salient features, such as definite sacroiliitis or psoriasis. More recently, a new classification of SpA has been proposed by the Assessment of SpondyloArthritis international Society (ASAS) based on the two main articular features of the disease: axial SpA (axSpA), for which chronic back pain is mandatory, and peripheral SpA, which requires peripheral arthritis, enthesitis, or dactylitis [4, 5]. In case of overlap, the 'main' articular manifestation drives the classification.

In addition to the articular manifestations and EAMs, patients with SpA may also suffer from conditions which do not belong to the concept of SpA but occur by chance or are related to the consequences of the disease or its treatment. Such co-existing diseases are referred to as comorbidities. Examples of comorbidities in SpA can be diabetes mellitus, chronic obstructive pulmonary disease (COPD) or malignancies. While the presence of EAMs may have consequences for the diagnosis of SpA, the presence of both EAMs and comorbidities may have consequences for the treatment, prognosis and outcome of the disease. Despite their clear importance, there are several gaps in our knowledge on the epidemiology and etiopathogenesis of both EAMs and other comorbidities, on their impact on health related quality of life (HROoL), and on valid approaches to measure co-existing diseases in outcome research. 


\section{Definitions index disease, comorbidity, multimorbidity, and EAMs}

The term 'comorbidity' was introduced by Feinstein in 1970, who defined a comorbidity as: 'any distinct additional entity that has existed or may occur during the clinical course of a patient who has the index disease under the study' [6]. The definition by Feinstein assumes that the comorbidity is seen from the perspective of an index disease which has a central position. In contrast, the term 'multimorbidity' usually refers to 'the co-occurrence of multiple chronic or acute diseases and medical conditions within one person', without any disease being more central than another [7]. One of the possible issues that may arise when a condition co-exists with an index disease, it to understand whether or not this co-existing disorder is part of the index disease or a distinct disease entity (and thus a 'true' comorbidity).

In SpA, as previously suggested by Moll, some co-existing diseases are so strongly associated with the articular disease, that they seem to be part of the index disease 'SpA' itself. These co-existing diseases are therefore nowadays referred to as EAMs and not as comorbidities. Notwithstanding, the term EAM is not exactly defined in SpA. Mielants et al. considered EAMs as all the conditions and symptoms in patients with SpA which are not directly related to the locomotor system but have a close link to SpA as a concept [8]. This definition is rather broad and, as a result, many conditions are considered as EAMs in different papers and textbooks on AS and SpA. Some sources distinguish two types of EAMs: EAMs related to the concept of SpA and EAMs not related to the concept of SpA [8]. EAMs related to the concept of SpA commonly occur in patients with SpA (20-60\% of patients), involve clinical inflammation, can occur at any moment of the disease evolution and their activity may fluctuate with the axial or peripheral joint symptoms. Furthermore, the activity and course of the disease can be modified by TNF-alpha blockers. Three conditions are considered as undisputable SpA-related EAMs: $A A U$, psoriasis, and IBD and therefore included in the new ASAS classification criteria for axial and peripheral SpA $[4,5]$. EAMs not related to the concept of SpA are rare (1\% of patients), are mostly subclinical in longstanding disease and are not related to joint symptoms. The effect of TNF-alpha blockers on non-concept related EAMs is unknown. Conditions that are frequently considered as non-SpA-concept related EAMs are located in the heart (e.g. conduction abnormalities), kidneys (e.g. IgA nephropathy) and the lungs (e.g. upper lobe fibrosis). The cause of most non-concept related EAMs in patients with SpA is unclear, but several hypotheses have been proposed. A relation with HLA-B27 has been recognized for some of these conditions such as for the cardiac manifestations [9]. Upper lobe fibrosis, on the other hand, may be explained by impaired ventilation as a result of reduced chest expansion, kyphosis, and rigidity of the thoracic spine [10]. As yet it is unclear whether these non-concept related EAMs can be considered as part of the disease concept or as comorbidity according to the definition of Feinstein. 
In summary, EAMs and comorbidities should be distinguished in SpA but for some diseases that co-exist in SpA it is difficult to exactly classify them as the link with the concept SpA has as yet not been fully elucidated. For the purpose of the present thesis, we will use the following definitions for co-existing diseases in patients with SpA or AS:

1. EAMs are co-existing conditions that are clearly part of the concept of SpA. They are pathogenetically related with SpA and may help to diagnose SpA. Following from this, undisputed EAMs comprise AAU, psoriasis and IBD.

2. Comorbidities are all distinct co-existing conditions that occur in patients with SpA but are not part of the concept of SpA, regardless of whether the comorbid condition is or is not related in some way to SpA.

For the present thesis, the above described 'non-concept related EAMs' will not be further addressed.

\section{Epidemiology and etiopathogenesis of EAMs and comorbidity in AS}

Several studies reported that EAMs frequently occur in patients with AS [11, 12]. It has been estimated that patients with AS have a 20-30\% chance of developing AAU; that $10-25 \%$ of patients with AS have concomitant psoriasis; and that IBD is diagnosed in $5-10 \%$ of patients with AS [12]. However, summary data on the prevalence of EAMs, clear data on the chronological relation between the development of AS and EAMs and data on the additional risk of EAMs compared with the general population are lacking.

Osteoporosis and cardiovascular disease are the most frequently studied comorbidities in patients with AS. Osteoporosis occurs frequently in patients with AS and can lead to spinal fractures. The prevalence of decreased bone mineral density ranges between 19 and $62 \%$ in patients with AS and the prevalence of spinal fractures ranges between 1 and $9 \%$ [13]. Further, in a systematic review it was shown that the prevalence of decreased BMD (51-54\%) and osteoporosis (13-16\%) is already high in patients with short disease duration (<10 years) [13]. Conflicting results exist about the risk of ischemic heart disease and myocardial infarction in patients with AS. While some studies find a 2-3 times increased risk compared with the general population, other studies report no increased risk [11, 14-16]. The possible increased prevalence of cardiovascular disease may be a result of the longstanding inflammation in AS, but also of treatment of AS, such as non-steroidal anti-inflammatory drugs (NSAIDs) $[17,18]$. Further, it has been proposed that the prevalence of traditional risk factors for cardiovascular disease is higher in patients with AS or that altered lifestyle in these patients accounts for the increased cardiovascular risk. However, because the studies on the cardiovascular risk in patients with AS are heterogeneous with regard to the study population and level of adjustment, clear conclusions about the cardiovascular risk cannot yet be drawn.

Similar to other chronic diseases, but less well studied, is the occurrence of depression as a comorbidity in AS. Studying depression is important, since it may have a large impact 
on health-related quality of life and participation in society. The prevalence of depressive symptoms is high in patients with AS and has been reported between 15 and 55\% when screening questionnaires for depression are used [19-23]. A recent study showed that $10 \%$ of patients with AS had a doctor-diagnosed depression in a 13-year observation period, compared to $6 \%$ to be expected in this period [24]. Depression in patients with AS may arise as an emotional response to pain and functional limitations [19, 22]. Another possible explanation is that one underlying biological mechanism, i.e. increased levels of cytokines such as TNF-alpha, may also contribute to the development of both AS and depression [25]. However, the relation between disease activity and depressive symptoms, and the potential improvement of depressive symptoms after initiation of effective treatment is only studied to a limited extent [26].

Large database studies have further suggested that patients with AS have a higher prevalence of other comorbidities, such as hypertension, diabetes mellitus, headache, COPD, liver disease, peptic ulcer disease, hepatitis, tuberculosis, deficiency anemia, and psychiatric disorders $[11,27]$. Their overall influence on HROLL and social role participation in patients with AS has not been systematically studied.

\section{Influence on outcome}

To measure co-existing diseases and in order to understand their impact on the course of the disease of interest, a validated approach is needed. Several studies revealed that the presence of a comorbidity is associated with mortality, HRQoL, functioning, health care costs, and quality of care $[28,29]$. Moreover, comorbidity adds considerably complexity to patient care, making diagnosis and treatment decisions more challenging [30]. As a result, it is increasingly recognized in SpA that comorbidities and/or EAMs should be taken into account when studying health outcomes. In observational studies, it is therefore essential to adjust for comorbidities and/or EAMs with an easy-to-use instrument to increase the validity of findings from epidemiologic studies. Different instruments are available to measure comorbidity and the choice of the instrument depends on the study outcome [31]. However, despite the importance of measuring comorbidity, no comorbidity instrument has been specifically validated for studies in patients with AS.

\section{Main aims}

In summary, it is still challenging to study the presence of co-existing disease in SpA and to embrace research in this area since current models of outcome assessment and care are usually designed for single diseases. This thesis aims to explore several aspects related to the epidemiology and outcome of EAMs and comorbidities in patients with SpA and AS.

The specific aims of the studies described here were: 
1. To assess the epidemiology of SpA and its phenotypes, and to explore which demographical and methodological factors (including classification criteria) influence reported prevalence rates

2. To gain insight into the epidemiology of EAMs in patients with $A S$, and vice versa into the frequency of articular SpA symptoms in patients with IBD

3. To validate the self-administered comorbidity index (SCO) for use in patients in AS

4. To improve our understanding about the risk of cardiovascular disease and depression in patients with AS

\section{Outline of this thesis}

In the first part of this thesis (chapter 2), the epidemiology of SpA is described. In the second part (chapter 3-5) various epidemiological aspects of EAMs in patients with AS and vice versa are discussed. The third part of this thesis (chapter 6-8) focuses on the relationship between comorbidities and AS.

\section{Part I: Epidemiology of SpA}

In part I of this thesis the epidemiology of SpA is described. The epidemiology of a disease is the most important determinant of the burden of a disease in a population. Several studies have been performed to investigate the prevalence and incidence of SpA and/or the subforms of SpA, such as AS and PsA. However, results of studies may differ as a result of differences in demographical and methodological characteristics, such as the geographic region or criteria used to classify patients. Therefore, chapter 2 comprises a systematic review and meta-regression analysis on the epidemiology of SpA, which also explored the impact of demographical and methodological issues (such as classification criteria) on reported prevalence numbers.

\section{Part II: Extra-articular manifestations}

Part II focuses on epidemiological aspects of the EAMs. Chapter 3 and 4 explore the epidemiology of EAMs in patients with AS, using different methodological approaches. Chapter 3 comprises a systematic literature review and meta-regression analysis of the available literature on the prevalence of EAMs in patients with AS. A meta-regression analysis explores clinical and methodological factors that may explain the variability in reported prevalence rates. Chapter 4 reports the results of a cohort study on the incidence and risks of EAMs in patients with AS as compared with matched controls in a large population-based database. The other way around, it is also interesting to gain insight into the frequency of joint symptoms in patients who are diagnosed with one of the conditions that are considered as EAMs in AS. In chapter 5, the self-reported prevalence of SpA-features in patients with IBD is studied and these numbers are compared with the actual referrals to a rheumatologist. 


\section{Part III: Comorbidities in ankylosing spondylitis}

In part III, different studies on comorbidities in AS will be presented. As abovementioned, comorbidities can affect the detection, prognosis, therapy and outcome of a single condition at any moment. Therefore, comorbidity should be considered as a confounder, effect modifier or predictor in studies on outcomes in AS. However, no comorbidity instrument has been evaluated for use in outcome studies in AS. Moreover, no instrument exists as yet that deals with the presence of EAMs in AS. In chapter 6, criterion and construct validity of the SCO are studied in patients with AS, the impact of comorbidity on different health outcomes is explored, and the absence of EAMs in such instruments is discussed. Chapter 7 and 8 address the question whether patients with AS are at increased risk of developing selected comorbidities in comparison with the general population, and the effect of treatment on this risk. In chapter 7, a population-based cohort study is described exploring the cardiovascular risk in patients with AS, with special attention to the role of NSAIDs on this risk. Chapter 8 explores the relationship between disease activity, anti-TNF-alpha treatment and depressive symptoms in patients with AS.

Finally, the findings described in this thesis are discussed in chapter 9 and summarized in chapter 10. 


\section{REFERENCES}

1. Moll JM, Haslock I, Macrae IF, et al. Associations between ankylosing spondylitis, psoriatic arthritis, Reiter's disease, the intestinal arthropathies, and Behcet's syndrome. Medicine (Baltimore). 1974/09/01 ed1974. p. 343-64.

2. Paramarta JE, Baeten D. Spondyloarthritis: from unifying concepts to improved treatment. Rheumatology (Oxford) 2013.

3. Braun ], Sieper ]. Building consensus on nomenclature and disease classification for ankylosing spondylitis: results and discussion of a questionnaire prepared for the International Workshop on New Treatment Strategies in Ankylosing Spondylitis, Berlin, Germany, 18-19 January 2002. Ann Rheum Dis 2002;61 Suppl 3:iii61-7.

4. Rudwaleit M, van der Heijde D, Landewe R, et al. The Assessment of SpondyloArthritis International Society classification criteria for peripheral spondyloarthritis and for spondyloarthritis in general. Ann Rheum Dis 2011;70:25-31.

5. Rudwaleit M, van der Heijde D, Landewe R, et al. The development of Assessment of SpondyloArthritis international Society classification criteria for axial spondyloarthritis (part II): validation and final selection. Ann Rheum Dis 2009;68:777-83.

6. Feinstein A. The pre-therapeutic classification of co-morbidity in chronic disease. ] Chronic Dis 1970;23: 455-68.

7. Van den Akker M. Comorbidity or multimorbidity: what's in a name? Eur ] Gen Pract 1996;2:65-70.

8. Mielants H, Van den Bosch F. Extra-articular manifestations. Clin Exp Rheumatol 2009;27:S56-61.

9. Lautermann D, Braun J. Ankylosing spondylitis--cardiac manifestations. Clin Exp Rheumatol 2002;20: S11-5.

10. Mercieca C, van der Horst-Bruinsma IE, Borg AA. Pulmonary, renal and neurological comorbidities in patients with ankylosing spondylitis; implications for clinical practice. Curr Rheumatol Rep 2014;16:434.

11. Bremander A, Petersson IF, Bergman S, et al. Population-based estimates of common comorbidities and cardiovascular disease in ankylosing spondylitis. Arthritis Care Res (Hoboken) 2011;63:550-6.

12. El Maghraoui A. Extra-articular manifestations of ankylosing spondylitis: prevalence, characteristics and therapeutic implications. Eur ] Intern Med 2011;22:554-60.

13. van der Weijden MA, Claushuis TA, Nazari T, et al. High prevalence of low bone mineral density in patients within 10 years of onset of ankylosing spondylitis: a systematic review. Clin Rheumatol 2012;31:1529-35.

14. Szabo SM, Levy AR, Rao SR, et al. Increased risk of cardiovascular and cerebrovascular diseases in individuals with ankylosing spondylitis: a population-based study. Arthritis Rheum 2011;63:3294-304.

15. Peters MJ, Visman I, Nielen MM, et al. Ankylosing spondylitis: a risk factor for myocardial infarction? Ann Rheum Dis 2010;69:579-81.

16. Brophy S, Cooksey R, Atkinson M, et al. No increased rate of acute myocardial infarction or stroke among patients with ankylosing spondylitis-a retrospective cohort study using routine data. Semin Arthritis Rheum 2012;42:140-5.

17. Mathieu S, Motreff P, Soubrier M. Spondyloarthropathies: an independent cardiovascular risk factor? Joint Bone Spine 2010;77:542-5.

18. Peters MJ, van der Horst-Bruinsma IE, Dijkmans BA, et al. Cardiovascular risk profile of patients with spondylarthropathies, particularly ankylosing spondylitis and psoriatic arthritis. Semin Arthritis Rheum 2004; 34:585-92.

19. Barlow JH, Macey S], Struthers GR. Gender, depression, and ankylosing spondylitis. Arthritis Care Res 1993; 6:45-51.

20. Baysal O, Durmus B, Ersoy Y, et al. Relationship between psychological status and disease activity and quality of life in ankylosing spondylitis. Rheumatol Int 2011;31:795-800.

21. Gunaydin R, Goksel Karatepe A, Cesmeli N, et al. Fatigue in patients with ankylosing spondylitis: relationships with disease-specific variables, depression, and sleep disturbance. Clin Rheumatol 2009;28: 1045-51. 


\section{Chapter 1}

22. Hakkou J, Rostom S, Aissaoui N, et al. Psychological status in Moroccan patients with ankylosing spondylitis and its relationships with disease parameters and quality of life. ] Clin Rheumatol 2011;17:424-8.

23. Hyphantis T, Kotsis K, Tsifetaki N, et al. The relationship between depressive symptoms, illness perceptions and quality of life in ankylosing spondylitis in comparison to rheumatoid arthritis. Clin Rheumatol 2013; 32:635-44.

24. Meesters J, Bremander A, Bergman S, et al. The risk for depression in patients with ankylosing spondylitis: a population-based cohort study. Arthritis Res Ther 2014;16:418.

25. Himmerich H, Fulda S, Linseisen J, et al. Depression, comorbidities and the TNF-alpha system. Eur Psychiatry 2008;23:421-9.

26. Ertenli I, Ozer S, Kiraz S, et al. Infliximab, a TNF-alpha antagonist treatment in patients with ankylosing spondylitis: the impact on depression, anxiety and quality of life level. Rheumatol Int 2012;32:323-30.

27. Kang JH, Chen YH, Lin HC. Comorbidity profiles among patients with ankylosing spondylitis: a nationwide population-based study. Ann Rheum Dis 2010;69:1165-8.

28. Fortin M, Bravo G, Hudon C, et al. Relationship between multimorbidity and health-related quality of life of patients in primary care. Qual Life Res 2006;15:83-91.

29. Ritchie C. Health care quality and multimorbidity: the jury is still out. Med Care 2007:45:477-9.

30. Gabriel SE, Michaud K. Epidemiological studies in incidence, prevalence, mortality, and comorbidity of the rheumatic diseases. Arthritis Res Ther 2009;11:229.

31. de Groot V, Beckerman H, Lankhorst G], et al. How to measure comorbidity. a critical review of available methods. J Clin Epidemiol 2003:56:221-9. 




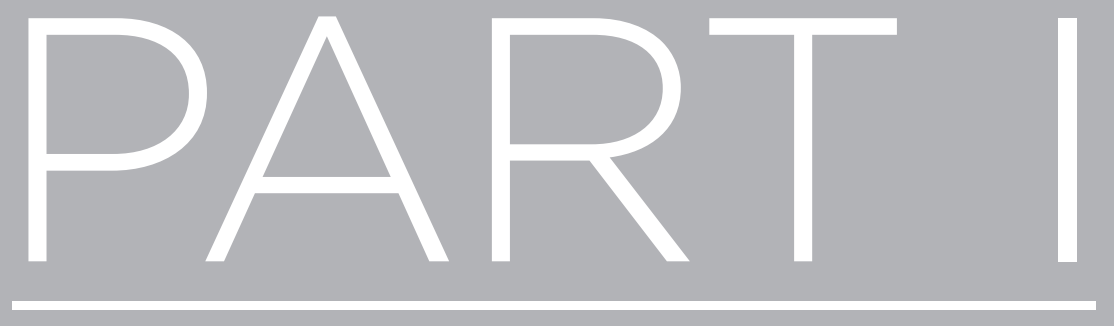

\section{Epidemiology of spondyloarthritis}

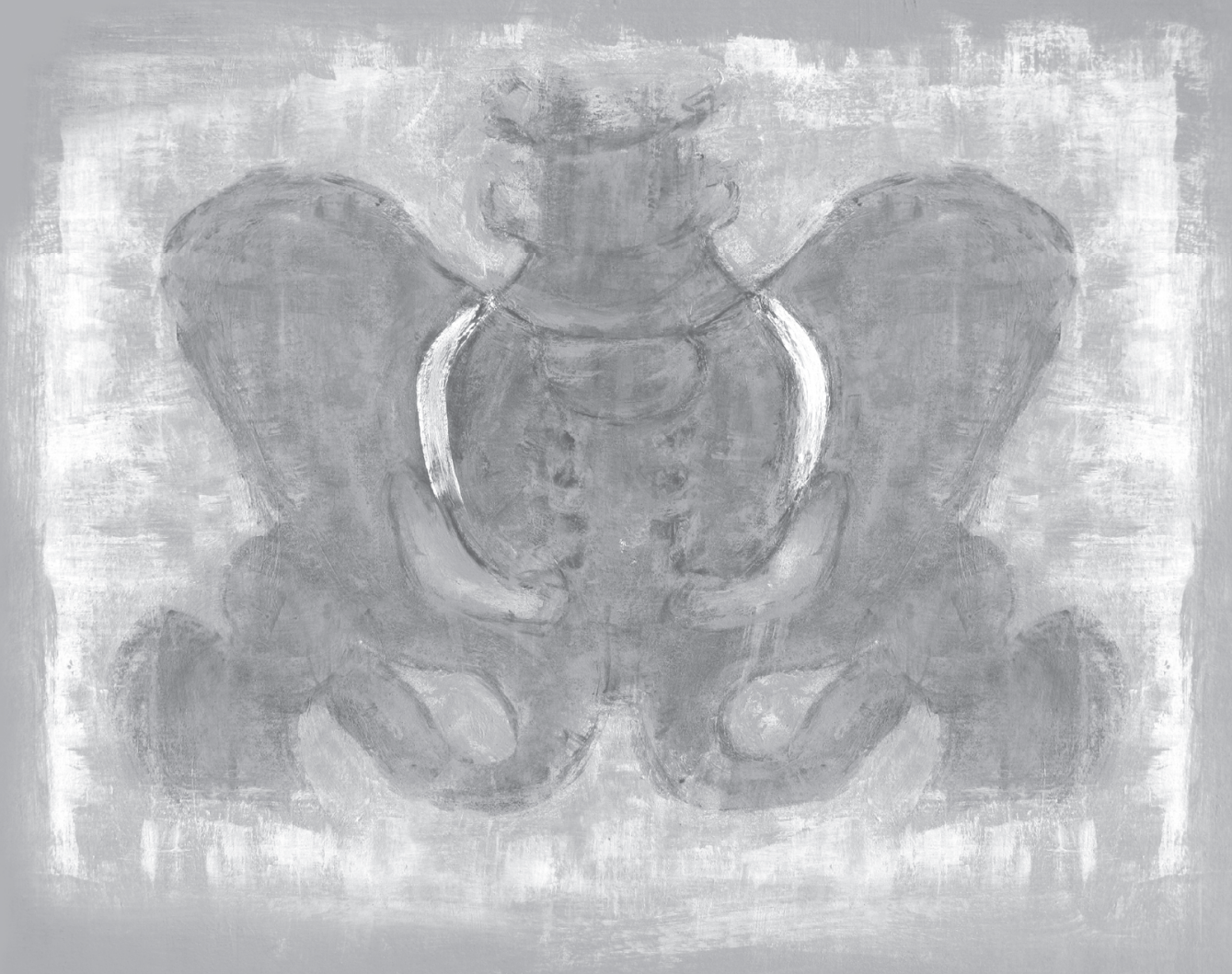





\section{CHAPTER 2 \\ The global prevalence of spondyloarthritis: a systematic review and meta-regression analysis}

Carmen Stolwijk, Marloes van Onna, Annelies Boonen, Astrid van Tubergen 


\section{ABSTRACT}

\section{Objective}

To summarize the prevalence of spondyloarthritis ( $\mathrm{SpA}$ ) and its subtypes in the general population, and to identify demographical and methodological characteristics that might explain heterogeneity in prevalence estimates.

\section{Methods}

A systematic literature search was performed to identify relevant articles. Risk of bias was assessed and data were extracted. Pooled prevalences were calculated. Potential sources of heterogeneity were explored by subgroup analysis and meta-regression analysis.

\section{Results}

In total 84 articles were included. The pooled prevalence of SpA, based on a random effects model, was 0.55\% (95\% Cl 0.37-0.77); for ankylosing spondylitis (AS) $0.18 \%$ (95\% $\mathrm{Cl} 0.15-0.23$ ); and for psoriatic arthritis (PsA) $0.15 \%$ (95\% Cl 0.12-0.18), but substantial heterogeneity was revealed $\left(I^{2}>99 \%\right)$. The following characteristics were significantly associated with variation in prevalence of SpA, AS and/or PsA: proportion of females, mean age of the sample, geographic area and setting (demographical characteristics); year of data collection, case finding, and case ascertainment (methodological characteristics). For the other SpA subgroups too few studies were available to conduct a meta-analysis, but prevalence estimates of reactive arthritis (range 0.0\%-0.2\%), SpA related to inflammatory bowel disease (range 0.0\%-0.1\%), and undifferentiated SpA (range 0.0\%-0.7\%) were generally low.

\section{Conclusion}

SpA is a common disease, but with large variation in reported prevalence estimates, which can partly be explained by differences in demographical and methodological characteristics. Particularly, geographic area as well as case finding account for a substantial part of the heterogeneity. 


\section{INTRODUCTION}

Since its establishment in the 1970s, the disease concept spondyloarthritis (SpA) has seen major developments with respect to identification and classification of the disease, measurement and prediction of outcome, and treatment options [1]. While SpA can be considered a condition itself, several subtypes can be distinguished, including ankylosing spondylitis (AS), psoriatic arthritis (PsA), SpA related to inflammatory bowel disease (IBD-SpA), reactive arthritis (ReA) and undifferentiated SpA (uSpA) [2]. More recently, the Assessment of SpondyloArthritis international Society (ASAS) proposed to distinguish axial from peripheral SpA, depending on the predominant articular manifestations of the disease, and developed new classification criteria [3, 4]. The treatment possibilities in patients with SpA have improved substantially in the last decade with the introduction of biologicals. On this line, it is relevant to gain insight into patterns of the prevalence of SpA and its subtypes, as this may contribute to our understanding of both the needs of healthcare systems - in terms of availability of healthcare resources and budgets - and the etiopathogenesis of the disease.

Considerable variation in the reported prevalence of SpA has already been recognized [5]. In particular, a wide range of estimates across geographic regions is found, which has classically been related to the presence of HLA-B27 [6]. However, other yet unknown demographical and methodological characteristics of the studies may also play a role in this variation. Demographical characteristics refer to for example the mean age of the sample, the male:female ratio, or the geographic region. Methodological characteristics include for example year of data collection, sampling frame, and case finding. Systematic approaches to gain insight into the epidemiology of SpA in the general population are limited [7]. No studies have been performed assessing and quantifying the effect of demographical and methodological characteristics on the prevalence of SpA. Therefore, the aims of the present study were 1 ) to perform a systematic review and meta-analysis of the literature on the prevalence of SpA and its subtypes, and 2) to identify demographical and methodological characteristics that might explain heterogeneity in prevalence.

\section{METHODS}

This systematic review was performed in accordance with the quality of reporting metaanalyses of observational studies (MOOSE) guidelines [8]. 


\section{Search strategy}

MEDLINE (via PubMed) and EMBASE (OVID) were searched between 1975-2014 July 1st for primary studies investigating the prevalence of SpA or one of its subtypes. The search strategy consisted of a combination of text words and controlled vocabulary terms (e.g. $\mathrm{MeSH}$ terms) relating to SpA and its subtypes, and to prevalence or epidemiology. The detailed search strategy is outlined in online Supplementary file 1 . Two reviewers independently reviewed titles and abstracts on eligibility criteria for inclusion, after which full-text was read. In addition, hand search of references was performed. If the full-text of the articles could not be retrieved, authors were contacted via email. In case of any discrepancies between the two reviewers, a third reviewer was consulted for final decision.

\section{Selection criteria}

Only original research studies reporting primary data on the prevalence of SpA or its subtypes in the general population were included. Studies were excluded if: 1) the study was not observational; 2) the study was published in a language other than English, French, German, Dutch, Spanish or Italian; 3) the study was only describing the prevalence of young-onset ( $<16$ years) SpA; 4 ) the article was not published in full-text; or 5 ) the study had a sample size $<1000$ subjects.

\section{Data extraction}

Data extraction was performed by two reviewers using a standardized form and included: study identification (first author, year of publication), and demographical as well as methodological characteristics. The demographical characteristics were mean age of the sample, proportion of females, geographic area and setting. Geographic area was subdivided into the following categories: Europe, North America, South America, Middle East and North Africa, Sub-Saharan Africa, Central Asia and Russia, South Asia, South-East Asia, East Asia, Oceania, and "Northern Artic indigenous communities". The last category was separated since it is known that the prevalence of HLA-B27 is higher in these populations $[9,10]$. The setting was subdivided into urban, rural, or a combination of both. The methodological characteristics were starting year of data collection (or alternatively publication year if not reported), sampling frame, case finding and case ascertainment. Sampling frame was subdivided into census, household register, convenience sample, general practitioner database, hospital database, register, or a list of specific group of subjects (e.g. employees of a company). Case finding was based on the procedure to identify cases and included self-reported symptoms, self-reported diagnosis, self-reported diagnosis followed by external confirmation (2-step approach diagnosis), self-reported symptoms followed by external confirmation (2-step approach symptoms), diagnosis by an expert, and hospital medical records or International Classification of Disease (ICD) codes. Case ascertainment was based on an external criterion used for case definition 
and was subdivided into clinical diagnosis (i.e. diagnosis by a physician) and classification criteria used for each SpA subtype, such as the modified New York criteria for AS, the European Spondyloarthropathy Study Group (ESSG) criteria, the ClASsification for Psoriatic Arthritis (CASPAR) criteria, or ASAS SpA criteria [3, 11-13]. Finally, data related to prevalence were extracted (raw data were extracted or the numerator and denominator were calculated). If a study presented age- and/or sex-specific estimates, these data were extracted, instead of the total count.

\section{Risk of bias assessment}

Two reviewers assessed independently the risk of bias for each included study, using a slightly modified version of a standardized tool by Hoy et al [14]. Online Supplementary file 2 shows the risk of bias tool including instructions on how each item was scored. We excluded the item "was the length of the shortest prevalence period for the parameter of interest appropriate" from the checklist, since this question is not applicable for a chronic disease, such as SpA.

\section{Data synthesis and analysis}

Because prevalence estimates were expected to be below 1\%, the values were transformed with the double arcsine transformation for meta-analysis and meta-regression [15]. The pooled prevalence was estimated by combining the transformed prevalence estimates using a random-effects model. Studies from Northern Artic indigenous communities were excluded from the meta-analysis, because the risk for SpA in this group is clearly different from the rest of the world, which would bias (i.e. overestimate) the results. The pooled prevalence and $95 \%$ confidence intervals $(\mathrm{Cl})$ were back transformed to prevalence estimates for ease of interpretation. The heterogeneity among studies was tested by the Cochran's Q test and the I² statistic [16].

Potential sources of heterogeneity were investigated by an exploratory subgroup analysis, using random effects analogous to one-way analysis of variance, in which groups of estimates were arranged according to potentially relevant demographical (mean age of the sample, proportion of females, geographic area, and setting) and methodological characteristics (year of data collection, study size, sampling frame, case finding, and case ascertainment). Studies from Northern Artic indigenous communities were excluded in the subgroup-analysis.

Univariable and multivariable meta-regression analyses were performed to explore associations between demographical and methodological characteristics and the prevalence. Variables with a $p$-value of $<0.20$ in the univariable analysis were entered into the multivariable model. A backward procedure was used, removing variables with a $p$-value of $>0.05$ in the multivariable model in order of significance. The following variables were tested: mean age of the sample, proportion of females, geographic area, setting, year of 
data collection, case finding, case ascertainment, and the dichotomized risk of bias criteria not yet covered by the previous variables. Due to collinearity, sampling frame was not included in the model. If data on age or sex were missing, respectively the mean or $50 \%$ were imputed. For the meta-regression analysis, SPSS macros were used (Metareg. sps and MetaF.sps) [17].

\section{RESULTS}

\section{Search results}

The database search yielded 9,240 studies (online Supplementary figure 1). After removing duplicates and title/abstract screening, 179 articles remained for full paper review. Two papers could not be retrieved, despite contacting the authors [18, 19]. After full-text reading, 80 articles were included. With hand search of references, 4 papers were added, leading to a total of 84 articles.

\section{Characteristics of included studies}

The prevalence of SpA was reported in 30 studies (100 age- and/or sex-specific estimates), of AS in 53 studies (179 estimates), of PsA in 35 studies (89 estimates), of ReA in 17 studies (67 estimates), of IBD-SpA in 4 studies (15 estimates), and of USpA in 15 studies (88 estimates). A detailed overview of all included studies is provided in online Supplementary file 3. A combined meta-analysis and meta-regression analysis could be performed for SpA, AS and PsA. Only a limited number of studies were available for ReA, IBD-SpA and USpA, therefore these results are described and summarized narratively.

Table 1 shows a summary of the main characteristics of studies included in the largest groups; SpA, AS and PsA.

\section{Risk of bias}

An overview of the risk of bias assessment is provided in online Supplementary Table 1. High risk of bias was most common for item 1 (representativeness of sample for the national general population) and item 2 (representativeness of sampling frame).

\section{Prevalence of spondyloarthritis}

The pooled population prevalence of SpA was $0.55 \%(95 \% \mathrm{Cl} 0.37-0.77)$, with high heterogeneity $\left(I^{2}=99.9 \%\right)$. Figure 1 shows the prevalence estimates of SpA according to different demographical and methodological characteristics. Mean age of the study population and geographic area contributed significantly to the observed heterogeneity. The prevalence of SpA was higher in studies from North America $(1.35 \%, 95 \% \mathrm{Cl} 0.44-2.79$, $\mathrm{n}=1$ study) and Europe $(0.54 \%, 95 \% \mathrm{Cl} 0.36-0.78)$ compared with South Asia $(0.22 \%$, 


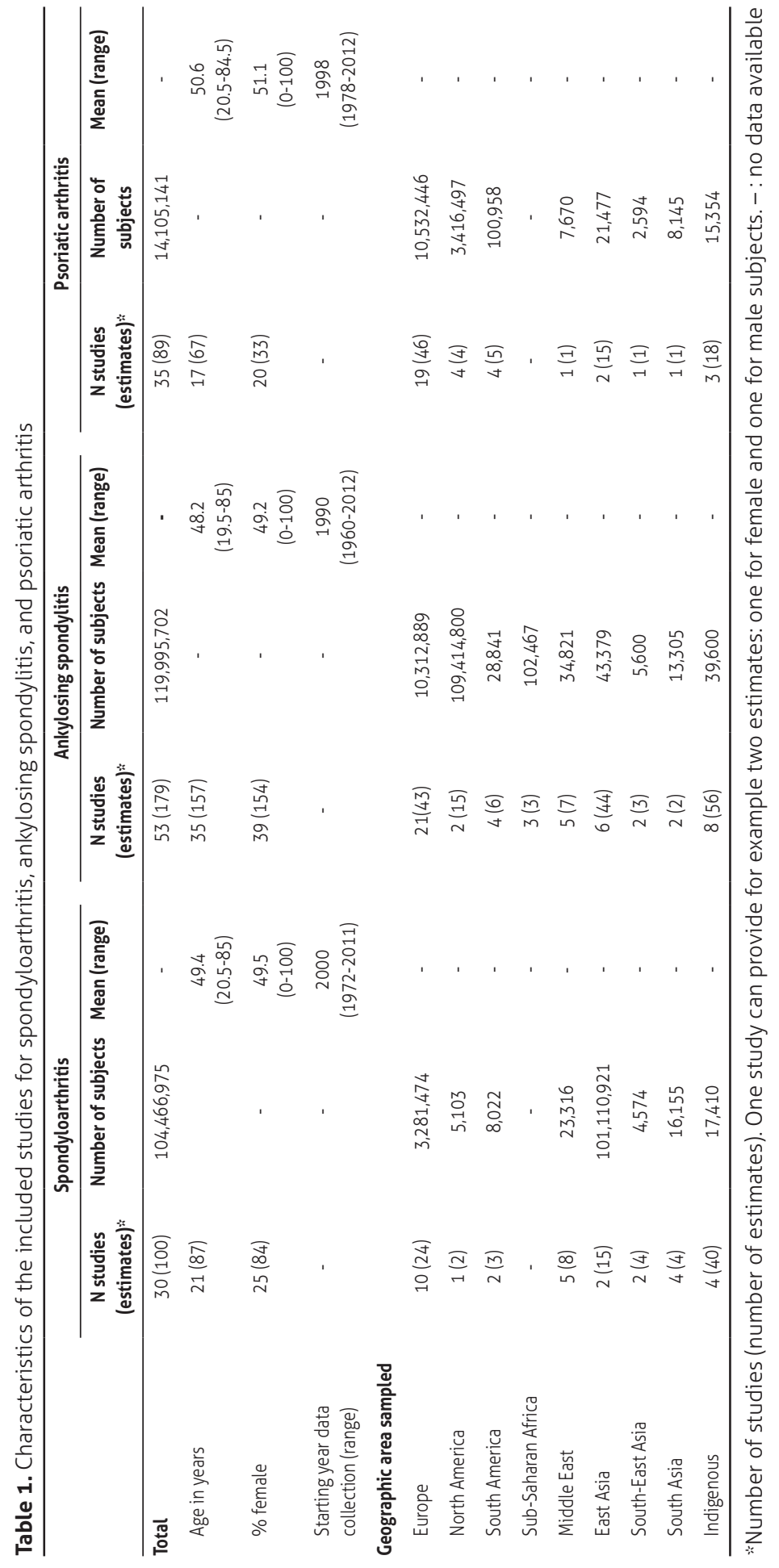




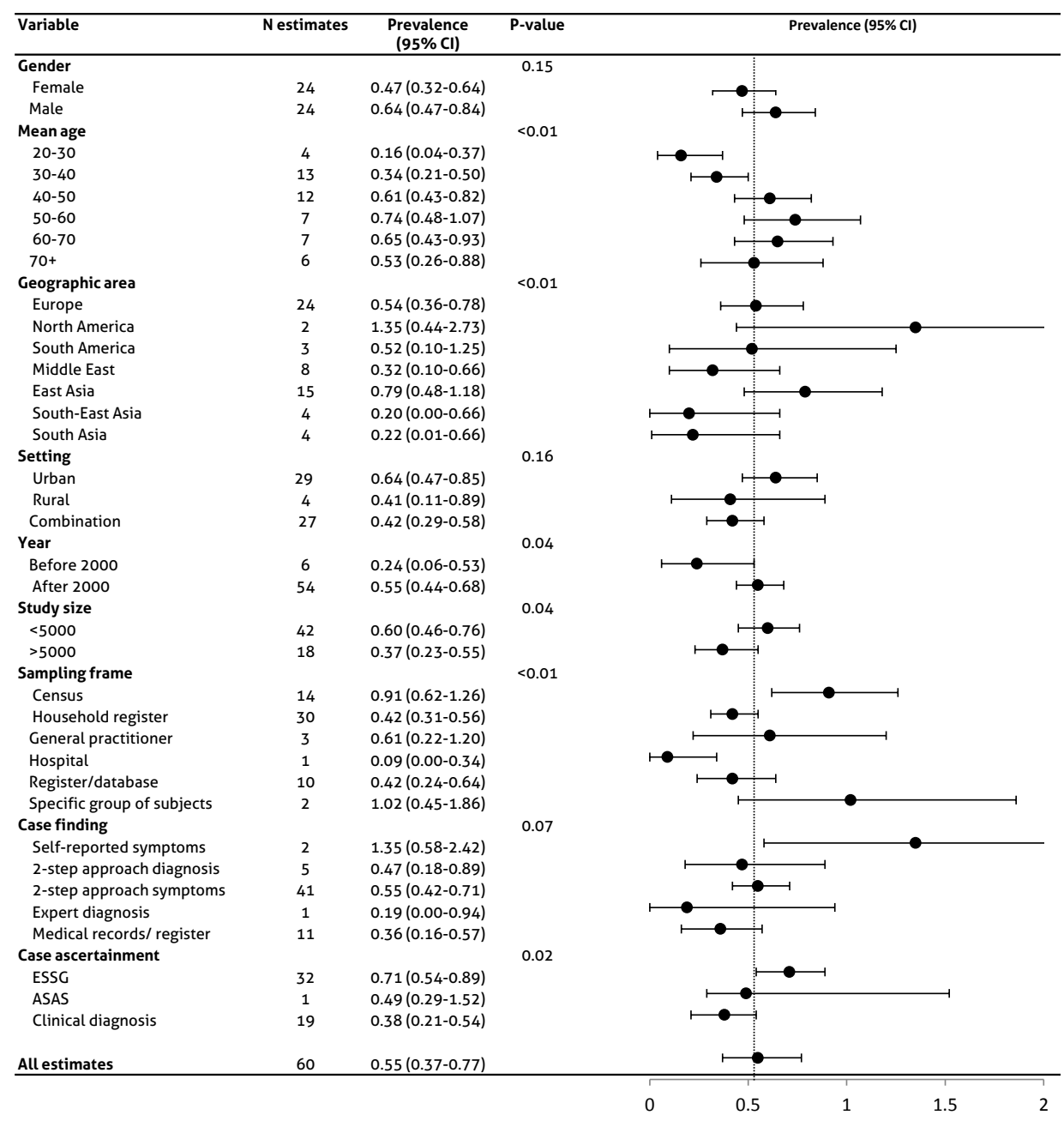

Figure 1 - Prevalence of spondyloarthritis grouped by demographical and methodological characteristics. Estimates of Northern Artic communities were not included in the subgroupanalysis. ESSG = European Spondyloarthropathy Study Group; ASAS = Assessment of Spondyloarthritis International Society

$95 \% \mathrm{Cl} 0.01-0.66)$ and South-East Asia (0.20\%, 95\% Cl 0.00-0.66). No studies from SubSaharan Africa, Central Asia and Oceania were available (Figure 2a). With respect to the sampling frame, true population studies reported higher prevalence estimates compared with hospital-based studies. The prevalence of SpA was also higher in more recent studies (year of data collection from 2000 onwards) and in smaller studies (<5000 subjects). Further, prevalence estimates were higher if based on the ESSG criteria compared with clinical diagnosis. Table 2 shows the results of the meta-regression analysis. The final model explained $51.8 \%$ of the heterogeneity. In this model, case finding $(P<0.01)$, a 
Table 2. Univariable and multivariable meta-regression analysis on the prevalence of spondyloarthritis

\begin{tabular}{|c|c|c|c|c|c|c|}
\hline \multicolumn{2}{|l|}{ Characteristic } & \multicolumn{3}{|c|}{ Univariable analysis } & \multicolumn{2}{|c|}{ Multivariable analysis } \\
\hline & & $\mathrm{B}(95 \% \mathrm{Cl})$ & P-value & $R^{2}$ & $\mathrm{~B}(95 \% \mathrm{Cl})$ & P-value \\
\hline \multicolumn{2}{|l|}{ Mean age } & $0.4 * 10^{-3}\left(-0.9 * 10^{-3} ; 0.2 * 10^{-2}\right)$ & 0.52 & 0.4 & NE & \\
\hline \multicolumn{2}{|l|}{$\%$ female } & $-0.4 * 10^{-3}\left(-0.8 * 10^{-3} ; 0.00\right)$ & 0.04 & 4.2 & $\begin{array}{c}-0.4^{*} 10^{-3} \\
\left(-0.6 * 10^{-3} ;-0.1 * 10^{-3}\right)\end{array}$ & 0.01 \\
\hline \multirow{7}{*}{$\begin{array}{l}\text { Region } \\
\text { Reference = Europe }\end{array}$} & North America & $0.08(-0.02 ; 0.12)$ & 0.11 & 30.3 & $0.10(0.01 ; 0.18)$ & 0.02 \\
\hline & South America & $-0.4 * 10^{-2}(-0.09 ; 0.08)$ & 0.93 & & $-0.07(-0.15 ; 0.00)$ & 0.06 \\
\hline & Middle-East & $-0.04(-0.09 ; 0.02)$ & 0.23 & & $-0.09(-0.15 ;-0.04)$ & $<0.01$ \\
\hline & East Asia & $0.03(-0.02 ; 0.08)$ & 0.23 & & $-0.01(-0.06 ; 0.04)$ & 0.66 \\
\hline & South-East Asia & $-0.06(-0.14 ; 0.02)$ & 0.15 & & $-0.08(-0.16 ;-0.01)$ & 0.02 \\
\hline & South Asia & $-0.05(-0.13 ; 0.02)$ & 0.16 & & $-0.10(-0.16 ;-0.03)$ & $<0.01$ \\
\hline & Northern Artic Indigenous & $0.10(0.06 ; 0.14)$ & $<0.01$ & & $0.18(0.11 ; 0.24)$ & $<0.01$ \\
\hline \multirow{2}{*}{$\begin{array}{l}\text { Setting } \\
\text { Reference =urban }\end{array}$} & Rural & $0.10(0.05 ; 0.16)$ & $<0.01$ & 15.8 & NS & \\
\hline & Combination & $-0.01(-0.05 ; 0.03)$ & 0.68 & & NS & \\
\hline \multicolumn{2}{|l|}{ Start data collection } & $\begin{array}{c}-0.3 * 10^{-2} \\
\left(-0.7 * 10^{-3} ;-0.5^{*} 10^{-2}\right)\end{array}$ & 0.01 & 6.2 & $\begin{array}{c}0.4 * 10^{-2} \\
\left(0.7 * 10^{-3} ; 0.7 * 10^{-2}\right)\end{array}$ & 0.02 \\
\hline \multicolumn{2}{|c|}{$\begin{array}{l}\text { Risk of bias item 1, representativeness target } \\
\text { population }\end{array}$} & $0.03(-0.05 ; 0.10)$ & 0.51 & 0.3 & NE & \\
\hline \multicolumn{2}{|c|}{$\begin{array}{l}\text { Risk of bias item 2, representativeness } \\
\text { sampling frame }\end{array}$} & $0.2 * 10^{-2}(-0.04 ; 0.05)$ & 0.92 & 0.0 & NE & \\
\hline \multicolumn{2}{|c|}{ Risk of bias item 3, random selection } & $-0.01(-0.08 ; 0.07)$ & 0.88 & 0.0 & NE & \\
\hline \multicolumn{2}{|c|}{ Risk of bias item 4, nonresponse bias } & $-0.07(-0.11 ;-0.02)$ & $<0.01$ & 7.9 & NS & \\
\hline \multicolumn{2}{|c|}{ Risk of bias item 5 , was a proxy used? } & $0.8 * 10^{-3}(-0.05 ; 0.05)$ & 0.97 & 0.0 & NE & \\
\hline \multicolumn{2}{|c|}{ Risk of bias item 6, validity of case definition* } & $-0.06(-0.12 ; 0.00)$ & 0.05 & 3.3 & NA & \\
\hline \multicolumn{2}{|c|}{$\begin{array}{l}\text { Risk of bias item 7, valid and reliable study } \\
\text { instrument }\end{array}$} & $0.02(-0.03 ; 0.07)$ & 0.53 & 0.3 & NE & \\
\hline \multicolumn{2}{|c|}{ Risk of bias item 8 , data collection mode } & $0.16(0.10 ; 0.22)$ & $<0.01$ & 19.6 & NS & \\
\hline \multicolumn{2}{|c|}{$\begin{array}{l}\text { Risk of bias item 9, numerator/denominator } \\
\text { appropriate }\end{array}$} & $-0.06(-0.14 ; 0.02)$ & 0.12 & 2.2 & NS & \\
\hline \multirow{4}{*}{$\begin{array}{l}\text { Case finding } \\
\text { Reference = medical } \\
\text { records }\end{array}$} & Two-step diagnosis & $0.13(0.08 ; 0.18)$ & $<0.01$ & 25.7 & $0.07(0.02 ; 0.11)$ & $<0.01$ \\
\hline & Two-step symptoms & $0.01(-0.03 ; 0.05)$ & 0.61 & & $0.08(0.04 ; 0.13)$ & $<0.01$ \\
\hline & Expert diagnosis & $0.11(0.02 ; 0.20)$ & 0.01 & & $0.08(0.01 ; 0.15)$ & 0.03 \\
\hline & Self-report & $0.09(-0.02 ; 0.19)$ & 0.11 & & $0.10(0.01 ; 0.18)$ & 0.02 \\
\hline \multirow{2}{*}{$\begin{array}{l}\text { Case ascertainment } \\
\text { Reference = clinical } \\
\text { diagnosis }\end{array}$} & ESSG & $0.08(0.03 ; 0.14)$ & $<0.01$ & 17.3 & NS & \\
\hline & ASAS & $0.02(-0.14 ; 0.18)$ & 0.80 & & NS & \\
\hline
\end{tabular}

"Risk of bias item 6 was not included in the multivariable analysis due to collinearity with case ascertainment ESSG = European Spondyloarthropathy Study Group criteria; ASAS = Assessment of SpondyloArthritis International Society criteria; $I C D=$ international classification of disease; $N E=$ not entered in multivariable model (because $p>0.20$ in univariable analysis); NS = not significant; NA $=$ not applicable (because of collinearity) 


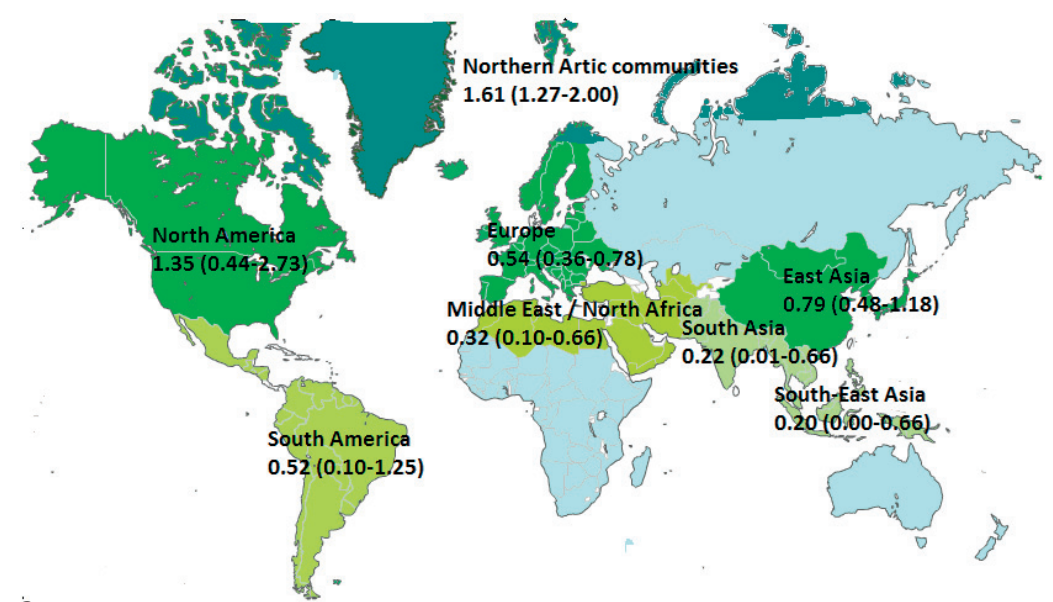

a

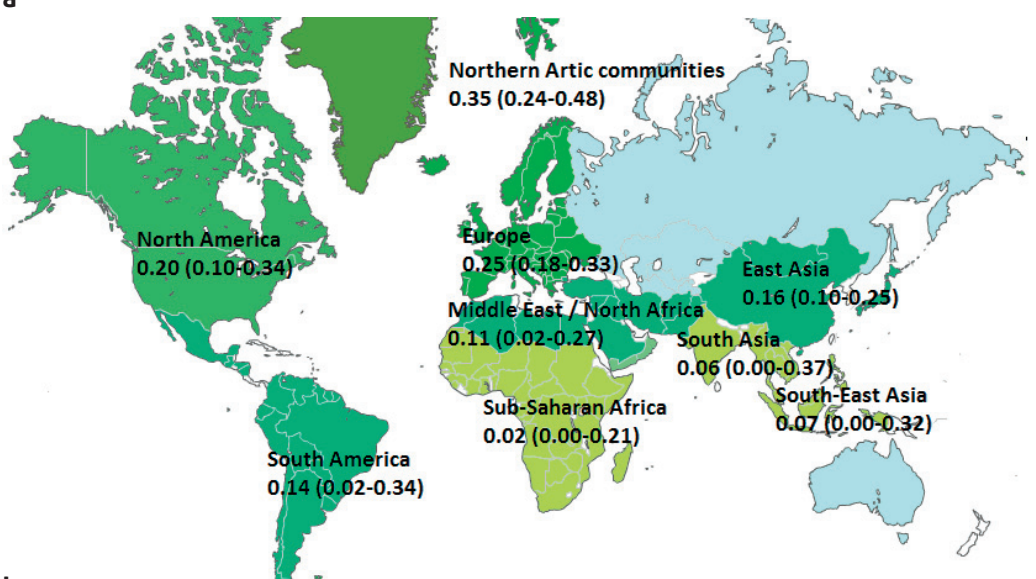

b

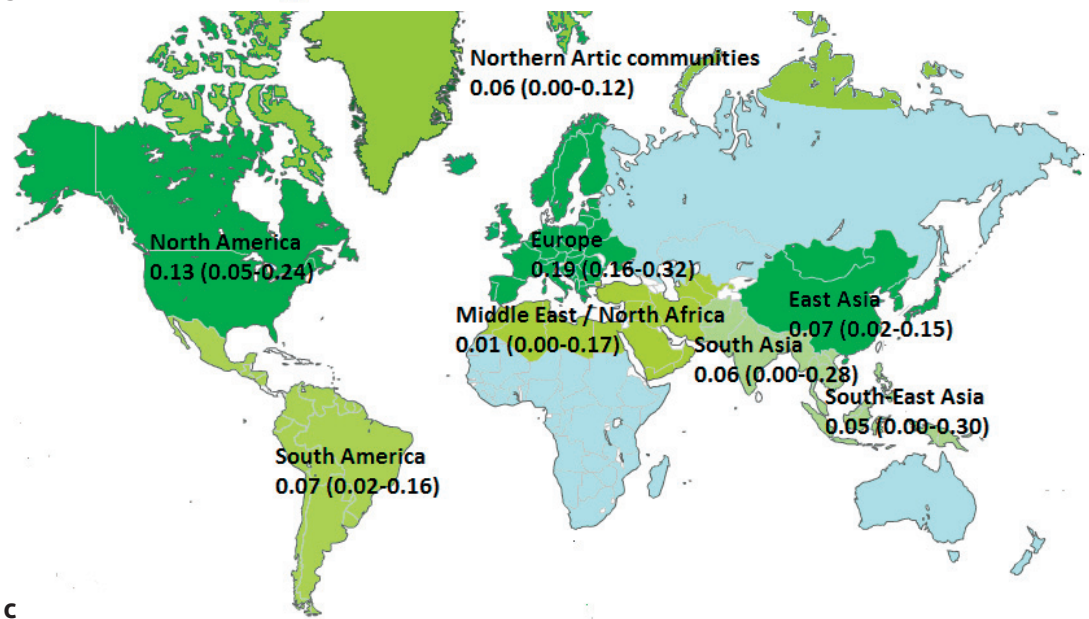

Figure $\mathbf{2}$ - Maps showing the global prevalence of spondyloarthritis (a), ankylosing spondylitis (b), and psoriatic arthritis (c). 
lower proportion of females $(p=0.01)$, geographic area $(p<0.01)$, and more recent year of data collection $(p=0.02)$ were positively associated with the prevalence of SpA.

\section{Axial and peripheral SpA}

Two studies reported the prevalence of axial and/or peripheral SpA according to the ASAS classification criteria $[20,21]$. In a large population-based cohort consisting of 20,625 employees of the French national electricity and gas company, a crude prevalence of SpA of $0.48 \%$ was found (0.36\% for axial SpA and $0.12 \%$ for peripheral SpA) [20]. In a study from the US, in which a sample of medical records of patients with chronic back pain were reviewed against the ASAS criteria and extrapolated to other US rheumatology practices, the prevalence of axial SpA was estimated at 0.70\% [21].

\section{Ankylosing spondylitis}

The pooled population prevalence of AS was $0.18 \%(95 \% \mathrm{Cl} 0.15-0.23)$. Heterogeneity was high $\left(I^{2}=99.0 \%\right)$. Figure 3 shows the pooled prevalence of AS stratified by subgroups. The prevalence of AS was higher in males compared with females. The prevalence was higher in rural populations and different across geographic areas, with the highest prevalence rates in studies from Europe and North America (Figure 2b). With regard to the methodological characteristics, the prevalence was higher in studies with $<5000$ subjects, different among sampling frames, and higher when patients were classified according to the (modified) New York or ASAS criteria compared with clinical diagnosis. The meta-regression analysis of the prevalence of AS is provided in online Supplementary table 2 . The final model explained $47.0 \%$ of heterogeneity. The prevalence of AS was higher in samples with a lower percentage of females $(p<0.01)$, different across geographic areas (higher prevalence rates in studies from North America, Europe and Northern Artic indigenous communities compared with all other geographic regions), and higher in samples from rural areas compared with urban areas $(p<0.01)$.

The prevalence was lower in samples in which cases were found by medical records compared with two-step symptoms approaches ( $p<0.01$ ), but higher in studies with high risk of bias for validity/reliability of the study instrument $(p<0.01)$.

\section{Psoriatic arthritis}

The pooled population prevalence of PsA was 0.15\% (95\% $0.12-0.17)$. Heterogeneity was high $\left(I^{2}=99.2 \%\right)$. The results of the subgroup analysis are shown in Figure 4 . The prevalence of PsA was significantly different across age groups and related to geographic area (Figure 2c). The highest prevalence was found in Europe $(0.19 \%, 95 \% \mathrm{Cl} 0.16-0.32)$ and the lowest in the Middle East $(0.01 \%, 95 \% \mathrm{Cl} 0.00-0.17)$. The prevalence of PsA was further significantly related to sampling frame and case finding. The highest prevalence of PsA was found when diagnosis was based on self-report $(0.26 \%, 95 \% \mathrm{Cl} 0.06-0.58)$. 


\begin{tabular}{|c|c|c|c|c|}
\hline Variable & $\begin{array}{c}\mathrm{N} \\
\text { estimates }\end{array}$ & $\begin{array}{c}\text { Prevalence } \\
(95 \% \mathrm{Cl})\end{array}$ & P-value & Prevalence $(95 \% \mathrm{Cl})$ \\
\hline Gender & & & $<0.01$ & \\
\hline Female & 48 & $0.12(0.09-0.17)$ & & \\
\hline Male & 51 & $0.31(0.23-0.41)$ & & $\mapsto$ \\
\hline Mean age & & & 0.44 & \\
\hline $20-30$ & 17 & $0.15(0.04-0.23)$ & & \\
\hline $30-40$ & 23 & $0.18(0.03-0.29)$ & & \\
\hline $40-50$ & 24 & $0.27(0.16-0.41)$ & & \\
\hline $50-60$ & 16 & $0.24(0.12-0.41)$ & & \\
\hline $60-70$ & 13 & $0.16(0.05-0.32)$ & & \\
\hline $70+$ & 12 & $0.14(0.03-0.33)$ & & \\
\hline Geographic area & & & $<0.01$ & \\
\hline Europe & 43 & $0.25(0.18-0.33)$ & & -1 \\
\hline North America & 15 & $0.20(0.10-0.34)$ & & \\
\hline South America & 6 & $0.14(0.02-0.34)$ & & \\
\hline Sub-Saharan Africa & 3 & $0.02(0.00-0.21)$ & & \\
\hline Middle East & 7 & $0.11(0.02-0.27)$ & & \\
\hline East Asia & 44 & $0.16(0.10-0.25)$ & & \\
\hline South-East Asia & 3 & $0.07(0.00-0.32)$ & & \\
\hline South Asia & 2 & $0.06(0.00-0.37)$ & & \\
\hline Setting & & & $<0.01$ & \\
\hline Urban & 45 & $0.20(0.14-0.28)$ & & -1 \\
\hline Rural & 14 & $0.52(0.35-0.72)$ & & $\longmapsto$ \\
\hline Combination & 64 & $0.12(0.08-0.16)$ & & \\
\hline Year & & & 0.75 & \\
\hline Before 2000 & 71 & $0.19(0.14-0.26)$ & & -1 \\
\hline After 2000 & 52 & $0.18(0.12-0.25)$ & & \\
\hline Study size & & & $<0.01$ & \\
\hline$<5000$ & 88 & $0.23(0.18-0.30)$ & & $\bullet-1$ \\
\hline$>5000$ & 32 & $0.11(0.06-0.17)$ & & \\
\hline Sampling frame & & & $<0.01$ & \\
\hline Census & 22 & $0.56(0.41-0.74)$ & & $\longmapsto$ \\
\hline Household register & 59 & $0.13(0.08-0.18)$ & & \\
\hline General practitioner & 2 & $0.36(0.05-0.92)$ & & \\
\hline Hospital & 7 & $0.11(0.02-0.22)$ & & \\
\hline Register/database & 27 & $0.13(0.08-0.21)$ & & \\
\hline Specific group of subjects & 3 & $0.28(0.08-0.59)$ & & \\
\hline Case finding & & & 0.22 & \\
\hline 2-step approach diagnosis & 5 & $0.24(0.06-0.54)$ & & $\bullet$ \\
\hline 2-step approach symptoms & 80 & $0.22(0.16-0.28)$ & & $\bullet$ \\
\hline Expert diagnosis & 5 & $0.13(0.01-0.26)$ & & 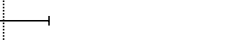 \\
\hline Medical records/ register & 33 & $0.13(0.07-0.20)$ & & \\
\hline Case ascertainment & & & .0 .03 & \\
\hline (mod) NY, Rome & 87 & $0.23(0.08-0.29)$ & & $\bullet$ \\
\hline ASAS & 2 & $0.32(0.00-1.11)$ & & \\
\hline Clinical diagnosis & 19 & $0.10(0.04-0.18)$ & & \\
\hline All estimates & 123 & $0.18(0.15-0.23)$ & & -1 \\
\hline
\end{tabular}

Figure 3 - Prevalence of ankylosing spondylitis grouped by demographical and methodological characteristics. Estimates of Northern Artic communities were not included in the subgroupanalysis. Mod NY = modified New York criteria; ASAS = Assessment of Spondyloarthritis International Society 


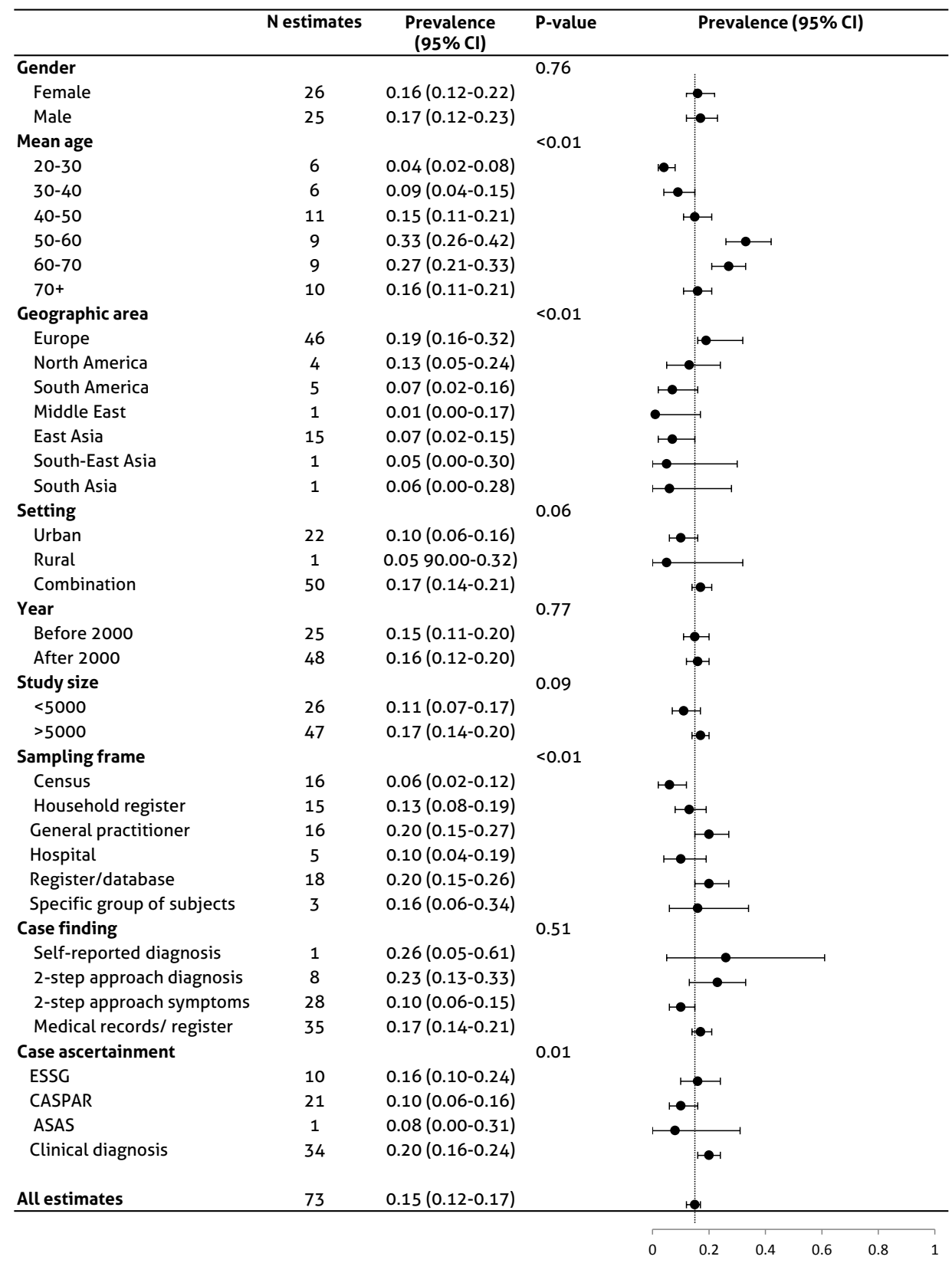

Figure 4 - Prevalence of psoriatic arthritis grouped by demographical and methodological characteristics. Estimates of Northern Artic communities were not included in the subgroupanalysis. ESSG = European Spondyloarthropathy Study Group; CASPAR= Classification for Psoriatic Arthritis; ASAS = Assessment of Spondyloarthritis International Society 
Online supplementary Table 3 shows the meta-regression analysis of the prevalence of PsA. The final model explained $44.7 \%$ of the total heterogeneity. A higher mean age of the sample was positively related to the prevalence of PsA $(p<0.01)$ and prevalence was significantly different across geographic areas. With respect to the methodological characteristics, prevalence was significantly higher when case finding was based on selfreport compared with medical records.

\section{Reactive arthritis}

The prevalence of ReA was reported in 17 studies: 6 from Europe, 1 from South-East Asia, 1 from Sub-Saharan Africa, and 9 from Northern Arctic indigenous populations. In Europe, the prevalence of ReA ranged from $0.03 \%$ in Greece [22] to $0.21 \%$ in Lithuania [23]. In a study from India, in which 8,145 individuals were interviewed, no cases of ReA were identified (prevalence 0\%) [24]. In a hospital-based study from Zimbabwe a prevalence of $0.001 \%$ was found [25]. In Northern Artic indigenous people, the prevalence ranged from $0.25 \%$ to $1.0 \%[26,27]$.

\section{Spondyloarthritis associated with inflammatory bowel disease}

The prevalence of IBD-SpA was reported in only 4 studies, all from Europe. Prevalence ranged from $0.0 \%$ in Greece [22] to $0.09 \%$ in Italy [28]. No formal criteria exist to classify IBD-SpA. In these 4 studies, classification was based on the ASAS-criteria [20], the ESSG criteria in combination with $\operatorname{IBD}[22,28]$, or ICD codes [29].

\section{Undifferentiated spondyloarthritis}

The prevalence of uSpA was reported in 15 studies. Different criteria were used to classify patients with uSpA. In most European studies, the prevalence ranged from $0.03 \%$ to $0.10 \%[20,22,23,28-30]$. In an urban population from Turkey, a prevalence of $0.56 \%$ was found [31]. In a study in blood donors from Germany a prevalence of $0.67 \%$ was reported [32]. In two Asian studies, the reported prevalences were $0.15 \%$ in a study from India [24] and $0.55 \%$ in a study from China [33]. In Northern Artic indigenous people, prevalences ranged from $0.20 \%$ to $1.3 \%[26,27,34-36]$.

\section{DISCUSSION}

In this systematic review, pooled population prevalence estimates were calculated for SpA (0.55\%, 95\%Cl 0.37-0.77), AS (0.18\%, 95\%Cl 0.15-0.23) and PsA (0.15\%, 95\%Cl 0.12-0.18). Heterogeneity across studies was high, therefore estimates should be interpreted with caution. For ReA, IBD-SpA and uSpA too few studies were available to conduct a meta-analysis and, therefore, results were only summarized. Prevalence estimates 
of ReA (range 0.0\%-0.2\%), IBD-SpA (range 0.0\%-0.1\%), and uSpA (range 0.0\%-0.7\%) were generally low.

This study is the first that pooled prevalences of SpA and its subtypes in the general population, and additionally investigated demographical and methodological characteristics influencing the estimates. Geographic area was in the multivariable meta-regression analysis one of the most important characteristics explaining heterogeneity in prevalence estimates of SpA. This variation might particularly be explained by genetic characteristics, such as HLA-B27. Independent of other characteristics, the highest prevalence estimates of SpA were found in Northern Artic indigenous communities, in which up to $50 \%$ of people have been reported to be HLA-B27 positive [9]. Further, higher prevalence estimates were found in studies from North America and Europe compared with Asia, Africa and the Middle East, corresponding with reported HLA-B27 prevalences in these areas [37]. A relatively high pooled prevalence of SpA was found in North America, however, the estimates came from a single study with a high risk of bias [38]. In this study, the prevalence of SpA was estimated according to the ESSG and the Amor criteria, resulting in estimates of $1.4 \%$ and $0.9 \%$ respectively [38]. This finding also illustrates that the choice of different classification criteria in epidemiological studies have a large impact on the reported prevalence. For reasons of comparability, we used estimates from this study based on the ESSG criteria when reporting the pooled prevalence in the present review, because these were also applied in most other studies [38]. Also the prevalence of SpA in East Asia was unexpectedly high (0.79\%), and seems to be explained by a high prevalence of USpA in China (0.55\%) [33].

Among the methodological characteristics explored, prevalence estimates of SpA were positively and independently related to the year of data collection with more recent studies reporting higher prevalences. This may either be a true increase in the prevalence of SpA, or, more likely, an increased awareness and recognition of SpA. Further, prevalence estimates were higher in studies where populations were screened for SpA compared with studies in which cases were identified from medical records. This finding suggests that there is under-recognition of SpA. In the subgroup analyses this is supported by the fact that sampling form census lists yielded the highest prevalence. With respect to case ascertainment, no significant difference was found between classification based on the ESSG criteria compared with clinical diagnoses. In the present review, too few studies using the new ASAS criteria were available to draw a conclusion. Future studies are needed to gain more insight into the impact of the ASAS criteria on the prevalence of SpA in population studies, although application of these criteria might be less feasible in large epidemiological studies, because of inclusion of HLA-B27 and MRI [39].

Similarly to SpA, also the prevalence of AS was significantly different among geographic areas in multivariable meta-regression and generally higher in regions of the northern hemisphere. Further, the prevalence of AS was higher in the male population. Tradition- 
ally, AS is considered as a disease predominantly occurring in males, although it has been shown that this may in part be an artefact induced by deficits in the diagnosis of AS in females [40-42]. Non-radiographic axial SpA, on the other hand, is as common in female as in male subjects, indicating that females develop structural changes later or less frequently than males $[39,43]$. In contrast, no difference in the prevalence of PsA in gender distribution was found with the multivariable meta-regression. The prevalence of PsA, however, was significantly related to age, and peaked in the age category between 50 and 60 years.

Some limitations of the present study should be addressed. First, we applied a language restriction; therefore language bias cannot be excluded. Second, because the majority of the studies came from Europe, geographical bias could have occurred. This undoubtedly influenced the pooled population prevalence, which should, therefore, be interpreted with caution. Further, even within the defined geographic areas, variation in prevalence might exist, for example between North and South Europe. However, further subdivision would have hampered the meta-regression analysis. Third, direct comparisons between the results of the meta-analyses and meta-regression analyses of SpA, AS, and PsA are hampered, because often populations from different studies were used. Last, meta-regression analysis itself has some limitations [44]. Results from meta-regressions are observational, and therefore, can suffer from bias by confounding. Patients' characteristics are based on group-averages, and the relationship on study level may not be the same as the relationship on patient level. Consistent with these concerns, high risk of bias was found for the items on representativeness of the sample and the sampling frame. Only a few studies were truly representative for the general population, which may hamper the generalization of the results.

In conclusion, this systematic review with meta-analysis summarized the prevalence of SpA and its subtypes. A large part of the heterogeneity could be explained by geographic characteristics. However, also other demographical and methodological characteristics, such as the proportion of females, year of data collection and case finding accounted for the observed variation. The results also suggested that there might still be an underdiagnosis of SpA. It is to be expected that better recognition of SpA will likely further increase the prevalence. High quality studies are needed to estimate the prevalence of axial and peripheral SpA in the general population, and to estimate the prevalence of SpA in developing countries. 


\section{REFERENCES}

1. Moll JM, Haslock I, Macrae IF, Wright V. Associations between ankylosing spondylitis, psoriatic arthritis, Reiter's disease, the intestinal arthropathies, and Behcet's syndrome. Medicine (Baltimore) 1974:343-64.

2. Dougados M, Baeten D. Spondyloarthritis. Lancet 2011;377:2127-37.

3. Rudwaleit M, van der Heijde D, Landewe R, et al. The Assessment of SpondyloArthritis International Society classification criteria for peripheral spondyloarthritis and for spondyloarthritis in general. Ann Rheum Dis 2011;70:25-31.

4. Rudwaleit M, van der Heijde D, Landewe R, et al. The development of Assessment of SpondyloArthritis international Society classification criteria for axial spondyloarthritis (part II): validation and final selection. Ann Rheum Dis 2009;68:777-83.

5. Stolwijk C, Boonen A, van Tubergen A, Reveille JD. Epidemiology of Spondyloarthritis. Rheum Dis Clin N Am 2012;38:441-76.

6. Mathieu A, Paladini F, Vacca A, et al. The interplay between the geographic distribution of HLA-B27 alleles and their role in infectious and autoimmune diseases: A unifying hypothesis. Autoimmun Rev 2009;8: 420-5.

7. Dean LE, Jones GT, MacDonald AG, et al. Global prevalence of ankylosing spondylitis. Rheumatology 2014; 53:650-7.

8. Stroup DF, Berlin JA, Morton SC, et al. Meta-analysis of observational studies in epidemiology: a proposal for reporting. Meta-analysis Of Observational Studies in Epidemiology (MOOSE) group. Jama 2000;283: 2008-12.

9. Gofton JP, Chalmers A, Price GE, Reeve CE. HL-A 27 and ankylosing spondylitis in B.C. Indians. J Rheumatol 1984;11:572-3.

10. Benevolenskaia LI, Erdes S, Krylov M, Chekalina NA. [The epidemiology of spondyloarthropathies among the native inhabitants of Chukotka. 2. The prevalence of HLA-B27 in the population and among spondyloarthropathy patients]. Ter Arkh 1994;66:41-4.

11. van der Linden S, Valkenburg HA, Cats A. Evaluation of diagnostic criteria for ankylosing spondylitis. A proposal for modification of the New York criteria. Arthritis Rheum 1984;27:361-8.

12. Dougados M, van der Linden S, Juhlin R, et al. The European Spondylarthropathy Study Group preliminary criteria for the classification of spondylarthropathy. Arthritis and rheumatism 1991;34:1218-27.

13. Rudwaleit M, van der Heijde D, Landewe R, et al. The development of Assessment of SpondyloArthritis international Society classification criteria for axial spondyloarthritis (part II): validation and final selection. Ann Rheum Dis 2009;68:777-83.

14. Hoy D, Brooks P, Woolf $A$, et al. Assessing risk of bias in prevalence studies: modification of an existing tool and evidence of interrater agreement. ] Clin Epidemiol 2012;65:934-9.

15. Barendregt J], Doi SA, Lee YY, Norman RE, Vos T. Meta-analysis of prevalence. ] Epidemiol Community Health 2013:67:974-8.

16. Higgins JP, Thompson SG. Quantifying heterogeneity in a meta-analysis. Stat Med 2002;21:1539-58.

17. Wilson D. METAREG for SPSS/Win 6.1 or higher MACRO. 2001; Available from: http://mason.gmu. edu/ dwilsonb/ma.html.

18. Chopra A, Patil J, Billempelly V, Relwani J, Tandle HS. Prevalence of rheumatic diseases in a rural population in western India: a WHO-ILAR COPCORD Study. J Assoc Physicians India 2001;49:240-6.

19. Mürden KD, Valkenburg HA, Hopper ]. The epidemiology of rheumatic diseases in Australia. APLAR ] Rheumatol 1992:402-210.

20. Costantino F, Talpin A, Said-Nahal R, et al. Prevalence of spondyloarthritis in reference to HLA-B27 in the French population: results of the GAZEL cohort. Ann Rheum Dis 2013

21. Strand V, Rao SA, Shillington AC, et al. Prevalence of axial spondyloarthritis in United States rheumatology practices: Assessment of SpondyloArthritis International Society criteria versus rheumatology expert clinical diagnosis. Arthritis Care Res (Hoboken) 2013;65:1299-306. 
22. Trontzas P, Andrianakos A, Miyakis S, et al. Seronegative spondyloarthropathies in Greece: a populationbased study of prevalence, clinical pattern, and management. The ESORDIG study. Clin Rheumatol 2005; 24:583-9.

23. Adomaviciute D, Pileckyte M, Baranauskaite A, et al. Prevalence survey of rheumatoid arthritis and spondyloarthropathy in Lithuania. Scand ] Rheumatol 2008;37:113-9.

24. Joshi VL, Chopra A. Is there an urban-rural divide? Population surveys of rheumatic musculoskeletal disorders in the Pune region of India using the COPCORD Bhigwan model. J Rheumatol 2009;36:614-22.

25. Lutalo SK. Chronic inflammatory rheumatic diseases in black Zimbabweans. Ann Rheum Dis 1985;44: 121-5.

26. Boyer GS, Lanier AP, Templin DW, Bulkow L. Spondyloarthropathy and rheumatoid arthritis in Alaskan Yupik Eskimos. ] Rheumatol 1990;17:489-96.

27. Boyer GS, Templin DW, Cornoni-Huntley JC, et al. Prevalence of spondyloarthropathies in Alaskan Eskimos. ] Rheumatol 1994;21:2292-7.

28. De Angelis R, Salaffi F, Grassi W. Prevalence of spondyloarthropathies in an Italian population sample: a regional community-based study. Scand J Rheumatol 2007;36:14-21.

29. Haglund $E$, Bremander AB, Petersson IF, et al. Prevalence of spondyloarthritis and its subtypes in southern Sweden. Ann Rheum Dis 2011;70:943-8.

30. Saraux A, Guillemin F, Guggenbuhl P, et al. Prevalence of spondyloarthropathies in France: 2001. Ann Rheum Dis 2005;64:1431-5.

31. Onen F, Akar S, Birlik M, et al. Prevalence of ankylosing spondylitis and related spondyloarthritides in an urban area of Izmir, Turkey. J Rheumatol 2008;35:305-9.

32. Braun J, Bollow M, Remlinger G, et al. Prevalence of spondylarthropathies in HLA-B27 positive and negative blood donors. Arthritis Rheum 1998;41:58-67.

33. Liao ZT, Pan YF, Huang JL, et al. An epidemiological survey of low back pain and axial spondyloarthritis in a Chinese Han population. Scand ] Rheumatol 2009;38:455-9.

34. Benevolenskaya LI, Boyer GS, Erdesz S, et al. Spondylarthropathic diseases in indigenous circumpolar populations of Russia and Alaska. Rev Rhum Engl Ed 1996;63:815-22.

35. Boyer GS, Lanier AP, Templin DW. Prevalence rates of spondyloarthropathies, rheumatoid arthritis, and other rheumatic disorders in an Alaskan Inupiat Eskimo population. J Rheumatol 1988;15:678-83.

36. Boyer GS, Templin DW, Lanier AP. Rheumatic diseases in Alaskan Indians of the southeast coast: high prevalence of rheumatoid arthritis and systemic lupus erythematosus. J Rheumatol 1991;18:1477-84.

37. Bakland G, Nossent HC. Epidemiology of spondyloarthritis: a review. Curr Rheumatol Rep 2013;15:351.

38. Reveille JD, Witter JP, Weisman MH. Prevalence of axial spondylarthritis in the United States: estimates from a cross-sectional survey. Arthritis Care Res (Hoboken) 2012;64:905-10.

39. van Tubergen A. The changing clinical picture and epidemiology of spondyloarthritis. Nat Rev Rheumatol 2014.

40. Gran JT, Husby G, Hordvik M. Prevalence of ankylosing spondylitis in males and females in a young middleaged population of Tromso, northern Norway. Ann Rheum Dis 1985:44:359-67.

41. Masi AT, Wilkins WR. Does male:female sex ratio in ankylosing spondylitis change with age? ] Rheumatol 1996;23:947-8.

42. Feldtkeller E, Bruckel J, Khan MA. Scientific contributions of ankylosing spondylitis patient advocacy groups. Curr Opin Rheumatol 2000;12:239-47.

43. Sieper ], van der Heijde D. Review: Nonradiographic axial spondyloarthritis: new definition of an old disease? Arthritis and rheumatism 2013;65:543-51.

44. Thompson SG, Higgins JP. How should meta-regression analyses be undertaken and interpreted? Stat Med 2002;21:1559-73. 




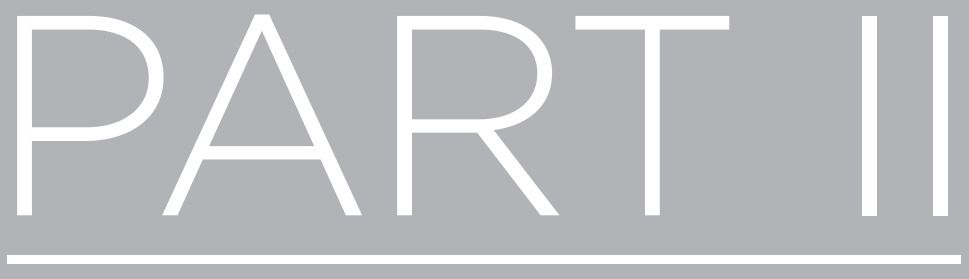

Extra-articular manifestations

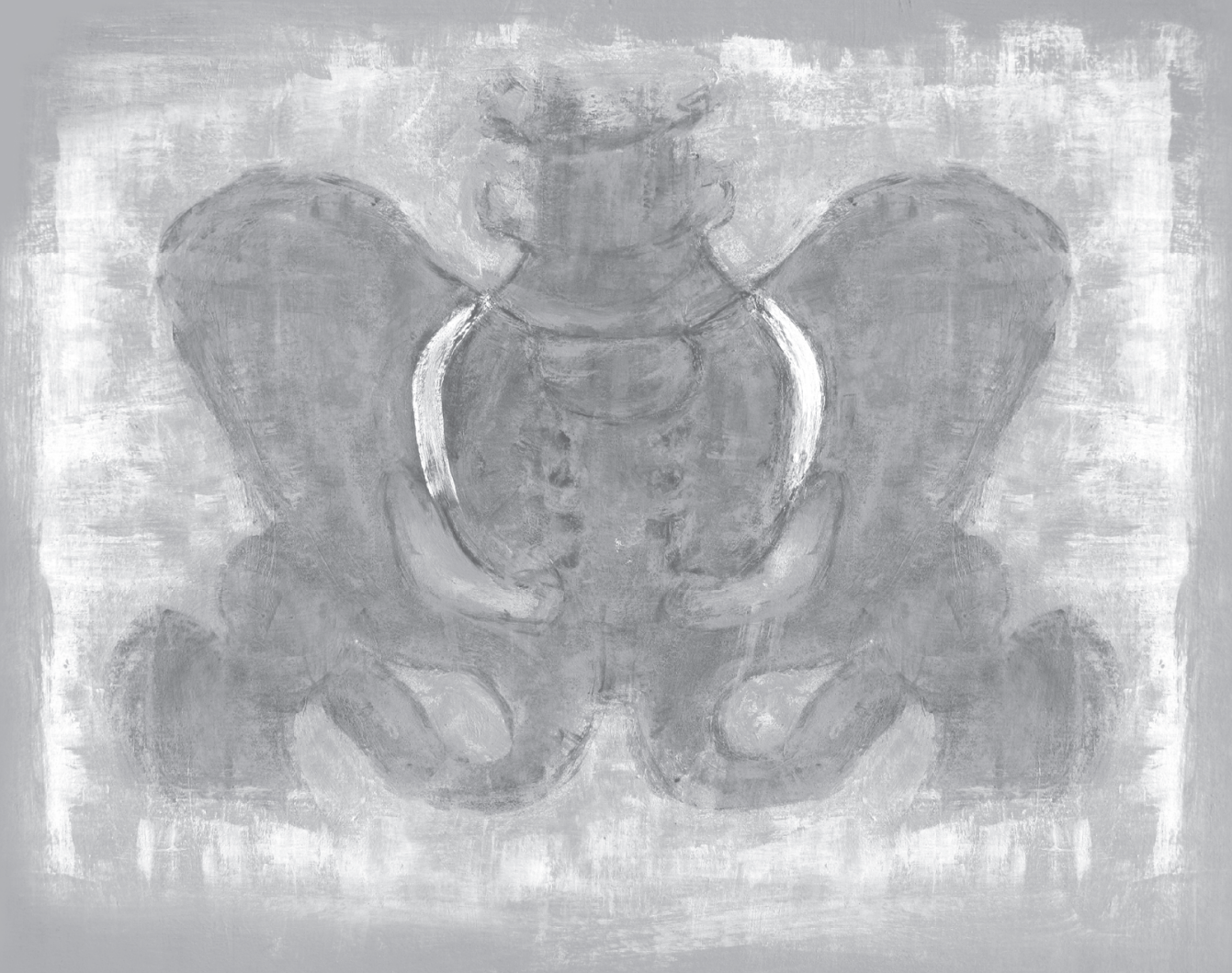





\section{CHAPTER 3}

\section{Prevalence of extra-articular manifestations in patients with ankylosing spondylitis: a systematic review and meta-analysis}

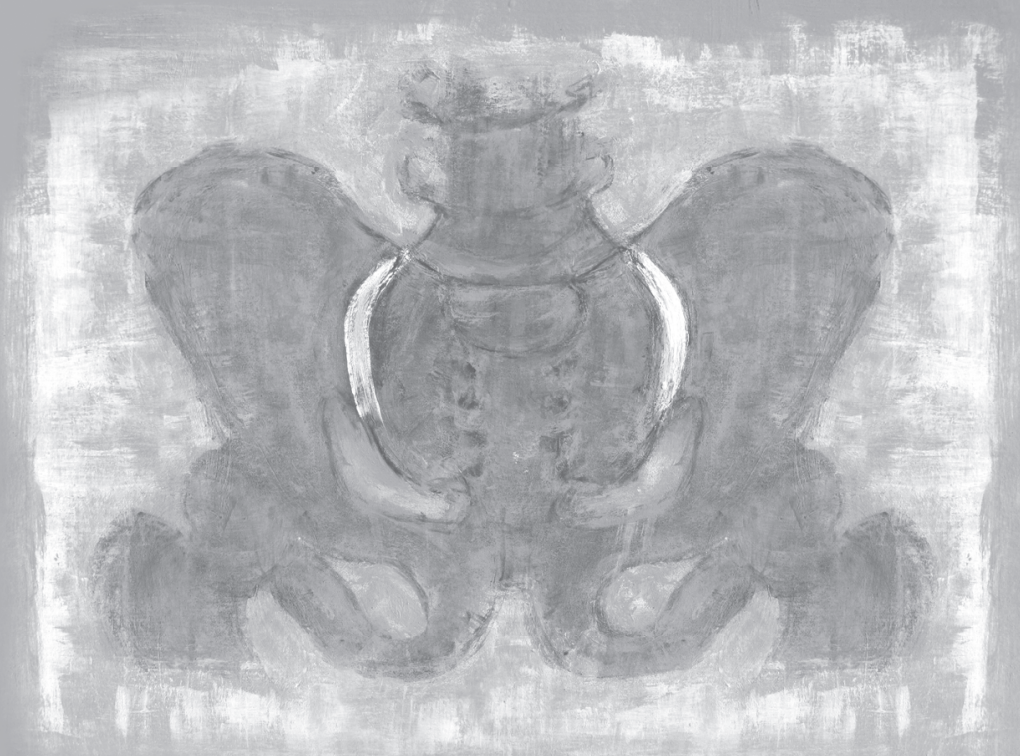

Carmen Stolwijk, Astrid van Tubergen, José Dionisio Castillo-Ortiz, Annelies Boonen 


\section{ABSTRACT}

\section{Objectives}

Uveitis, psoriasis and inflammatory bowel disease (IBD) are common extra-articular manifestations (EAMs) in patients with ankylosing spondylitis (AS); however, summary data of reported prevalence are lacking. The aim of the present study was to summarize the prevalence of EAMs among patients with AS and to identify underlying factors to explain potential heterogeneity of prevalence.

\section{Methods}

A systematic literature search was performed (Medline, Embase and Cochrane Library) to identify relevant articles. Risk of bias was assessed and data were extracted. Pooled prevalences were calculated. Potential sources of any observed clinical or methodological heterogeneity in the estimates were explored by subgroup- and meta-regression analysis.

\section{Results}

In the 156 selected articles, 143 reported the prevalence of uveitis (44372 patients), 56 of psoriasis (27626 patients) and 69 of IBD (30410 patients). Substantial heterogeneity was observed in prevalence estimates among all EAMs ( $\left.I^{2}=84-95 \%\right)$. The pooled prevalence of uveitis was $25.8 \%$ (95\% Cl 24.1-27.6), and was positively associated in multivariable meta-regression with disease duration ( $\beta 0.05,95 \% \mathrm{Cl} 0.03-0.08)$ and random selection of patients $(\beta-0.24,95 \% \mathrm{Cl}-0.04 ;-0.43)$. The pooled prevalence of psoriasis was $9.3 \%$ (95\% Cl 8.1-10.6). The pooled prevalence of IBD was $6.8 \%$ (95\% Cl 6.1-7.7) and was positively associated with the percentage of females in the studies $(\beta 0.02,95 \%$ $\mathrm{Cl}$ 0.00-0.03). Geographic area was associated in multivariable meta-regressions with prevalence of all EAMs.

\section{Conclusion}

EAMs are common in patients with AS. The large heterogeneity between studies can be partly explained by differences in clinical as well as methodological characteristics. 


\section{INTRODUCTION}

Ankylosing spondylitis (AS) is the prototype of a group of diseases referred to as spondyloarthritis (SpA) [1]. This group of diseases share genetic, radiographic and clinical features including an increased prevalence of acute anterior uveitis (AAU), psoriasis and inflammatory bowel disease (IBD), the so-called extra-articular manifestations (EAMs) [2]. The attention to EAMs in AS has increased in the past years. First, the presence of AAU, psoriasis and IBD in patients with (inflammatory) back pain may help to make a diagnosis of axial SpA (axSpA) [3]. Next, the presence of one or more EAMs can affect the prognosis of AS and may influence health outcomes including quality of life, work outcome and health resource utilization [4-7]. Moreover, EAMs in patients with AS may influence treatment decisions [2]. Last, insight in the co-existence of EAMs in patients with AS might help to unravel the role of genetic and epigenetic phenomena in the pathophysiology of inflammatory processes involved in SpA [8].

As a consequence, epidemiological data on the prevalence of EAMs have become increasingly important for clinicians and researchers when trying to understand disease patterns. However, although many studies report the occurrence of the EAMs, only one systematic literature review (SLR), providing only descriptive results and no meta-analysis, is currently available on the prevalence of AAU in SpA [9]. Furthermore, prevalences of the individual EAMs that are reported in available studies vary substantially. It is currently unclear whether factors can be identified which may explain this variation.

The aims of the present study were 1) to summarize and evaluate the published estimates for the prevalence of EAMs among patients with AS, and 2) to explore the effect of various clinical and methodological factors on reported prevalence. We hypothesized that the prevalences of all EAMs would be associated with disease duration and with geographic area, the latter being associated with differences in distribution of HLA-B27 positivity. Further, it was hypothesized, that the prevalence of EAMs would be associated with the method of ascertainment, with higher prevalences in studies based on self-report.

\section{METHODS}

An SLR with meta-analysis and meta-regression analysis was performed in order to obtain best possible estimates of the prevalence of AAU, psoriasis and IBD in patients with AS.

\section{Search strategy and selection criteria}

An SLR was conducted in MEDLINE (January 1, 1984 to August 1, 2012), EMBASE (January 1, 1989 to August 1, 2012) and the Cochrane Central Register of Controlled trials (CENTRAL). The search strategy, developed with assistance of an experienced librarian 
and adapted for each database (see online supplementary text 1), consisted of a combination of free terms and controlled vocabulary terms relating to AS and to the EAMs. Additionally, 'snowballing' was used to augment the search sensitivity including reference tracking, checking the search results of other SLRs on AS, which were performed by our research team during the same period, and personal knowledge of the authors. There were no restrictions in language of the papers. One author selected potentially relevant articles after reading the title, keywords and abstract. If in doubt, the full article was read. For inclusion, studies had to meet the following criteria: presenting primary data including the prevalence of AAU, psoriasis and/or IBD in patients ( $\geq 16$ years) with a clinical diagnosis of AS. The prevalence of EAMs did not have to be the main objective of the study and as a consequence, also studies in which EAMs were reported in the baseline description of the study population were considered. Studies including patients with other diagnoses besides AS were only eligible if the results from patients with AS were presented separately. Excluded were abstracts not (yet) published as full manuscripts; opinion or discussion papers; case reports and studies including $<20$ patients. If more than one article reported the same cohort of patients, only the first published article was included.

\section{Risk of bias}

Each study was independently rated by two reviewers for risk of bias based on the Methodological Evaluation of Observational research (MORE) checklist, which was adapted to the specific research question [10]. External validity is defined as the extent to which the results of a study can be generalized to the target population and comprised for the present study the following questions: 1) 'Was the sampling frame a close representation of the target population?' 2) 'Was an appropriate case definition for AS used?' 3) 'Was some form of random selection used to select the sample?', and 4) 'Was the likelihood of nonresponse bias minimal?'. The internal validity is defined as the possible amount of error in measuring the conditions and included the following question: 5) 'Was an acceptable case definition for the EAM used?' [10]. For each criterion, three options were possible: "+" = low risk of bias, "-"= possible risk of bias or "?" = risk of bias unclear due to poor reporting. The predefined rating procedure is shown in the online supplementary table 1 . In case of any discrepancies between the two reviewers, a third reviewer was consulted for final decision. For each criterion a score of " 1 " (low risk of bias) or " 0 " (possible or unclear risk of bias) was given. Finally, a composite score was calculated as the sum of the score on the individual criteria. The criterion 'response rate' was not considered in the composite score because too many scores were not reported. 


\section{Data extraction}

Data extraction of the first 20 articles was performed by two reviewers using a standardized data extraction form. Because the agreement on this data extraction was $100 \%$, the remaining of the data extraction was performed by only one reviewer. Data extraction included (if available): study identification (first author, journal, year of publication), study characteristics (study design, geographic area, sample size), patient characteristics (average age of patients, percentage of females, percentage of HLA-B27 positive patients, mean disease duration of AS [years since diagnosis]), and study results (number of patients with (a history of) AAU, psoriasis or IBD). A native speaker or translator was asked for help with articles published in other languages than English, Dutch, Spanish or Portuguese.

\section{Statistical analysis}

The pooled prevalence with 95\% confidence interval (CI) of each EAM was estimated using inverse variance weighting. The heterogeneity among studies for each EAM was tested by Cochran's $\mathrm{Q}$ test and the $\mathrm{I}^{2}$ statistic, the latter describing the percentage of variation across studies [11]. Whenever heterogeneity was high $\left(I^{2}>50 \%\right)$, random effects models were used [12]. For all analyses, logit transformation was applied to the prevalence estimates to improve their statistical properties [13]. The final pooled logit results and $95 \% \mathrm{Cls}$ were back-transformed to prevalence estimates for ease of interpretation. Potential sources of heterogeneity were first investigated by an exploratory subgroup analysis, using random effects analog to one-way ANOVA, in which groups of studies were arranged according to potentially relevant clinical characteristics (disease duration; percentage of females; percentage of HLA-B27 positive patients; and geographic area, all subdivided in quartiles) and methodological characteristics (study design; sample size; sampling frame; case definition for AS; random selection of patients; response rate; case definition for EAM; and the composite score for risk of bias). Second, random-effects meta-regression analysis via iterative maximum likelihood was performed to explore associations between the clinical- and methodological characteristics (moderators) and the outcome [14]. The advantage of meta-regression analysis, compared to subgroup analysis, is the possibility of evaluation of continuous moderators and evaluation of more than one moderator simultaneously. In the meta-regression analysis, the same clinical characteristics were entered as in the subgroup analysis, but now as continuous variables, as appropriate. The influence of methodological characteristics was evaluated by the individual risk of bias criteria (low risk versus possible risk of bias) as well as study design and sample size.

Variables with a p-value of $<0.30$ in the univariable analysis were entered in the multivariable model. Multicollinearity between variables was checked by computing variance inflation factors (VIF) before inclusion into the model [15]. A backward procedure was 
used, removing variables with a p-value of $>0.05$ in the multivariable model in order of significance, until the best-fitting model was identified. For categorical variables, the $\mathrm{p}$-value was calculated for all dummy variables together ( $\mathrm{R}^{2}$ change). Interactions between the variables were explored by additional analyses that included the main effect variables and the interaction term for each interaction studied. All statistical analyses were conducted using SPSS 18.0. For the meta-regression analysis, SPSS macros were used (Metareg.sps and MetaF.sps) [16].

\section{RESULTS}

\section{Search}

The search in databases resulted in 5898 articles (Figure 1). After excluding duplicate articles and screening on title and abstract, 385 articles remained for full-paper review. Three full text articles could not be retrieved, despite efforts to contact the corresponding author. After reading the full-text of the 382 articles, 94 articles were included. The main reasons for exclusion were no primary data reported or no data on patients with AS. The additional search resulted in inclusion of another 62 articles. In total, 156 articles were included.

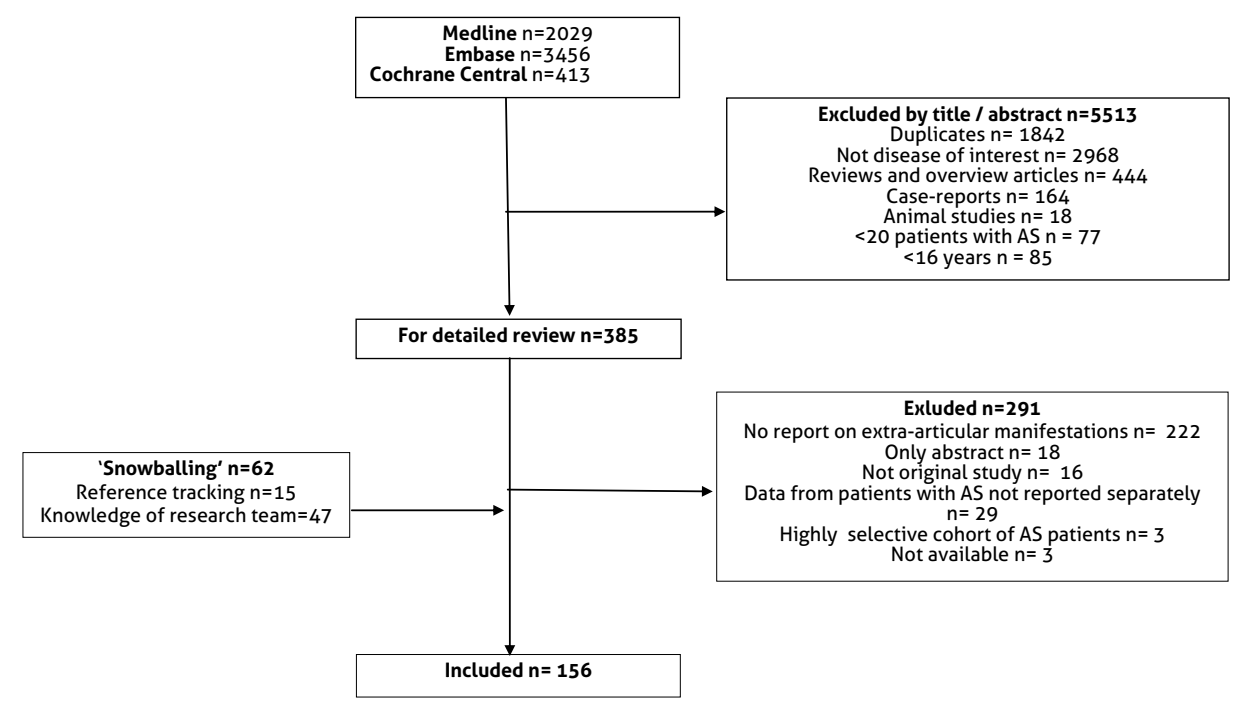

Figure 1 - Flow-diagram of systematic literature search. $n$ = number of studies

\section{Characteristics of the included studies}

The table of individual studies with full references and detailed characteristics is available in the online supplementary table 2. A summary of the clinical characteristics of 
Table 1. Clinical characteristics of the 156 included studies specified for each extra-articular manifestation

\begin{tabular}{|c|c|c|c|c|c|c|c|c|c|}
\hline & \multicolumn{3}{|c|}{ AAU } & \multicolumn{3}{|c|}{ Psoriasis } & \multicolumn{3}{|c|}{ IBD } \\
\hline & $\begin{array}{c}\text { Number } \\
\text { of studies } \\
(\%)^{*}\end{array}$ & $\begin{array}{c}\text { Number of } \\
\text { patients } \\
(\%)^{*}\end{array}$ & $\begin{array}{l}\text { Weighted } \\
\text { Mean } \\
\text { (SD)‡ }\end{array}$ & $\begin{array}{c}\text { Number } \\
\text { of studies } \\
(\%)^{*}\end{array}$ & $\begin{array}{l}\text { Number of } \\
\text { patients } \\
(\%)^{*}\end{array}$ & $\begin{array}{l}\text { Weighted } \\
\text { Mean } \\
\text { (SD) }\end{array}$ & $\begin{array}{c}\text { Number } \\
\text { of studies } \\
(\%)^{*}\end{array}$ & $\begin{array}{l}\text { Number of } \\
\text { patients } \\
(\%)^{*}\end{array}$ & $\begin{array}{c}\text { Weighted } \\
\text { Mean } \\
\text { (SD) } \ddagger\end{array}$ \\
\hline Total & 143 & 44372 & - & 56 & 27626 & - & 69 & 32341 & - \\
\hline Age in years & $\begin{array}{c}112 \\
(78.3 \%)\end{array}$ & $\begin{array}{c}33650 \\
(75.8 \%)\end{array}$ & $43.1(5.9)$ & $\begin{array}{c}49 \\
(87.5 \%)\end{array}$ & $\begin{array}{c}20267 \\
(73.4 \%)\end{array}$ & $45.3(4.6)$ & $\begin{array}{c}61 \\
(88.4 \%)\end{array}$ & $\begin{array}{c}24836 \\
(76.8 \%)\end{array}$ & $45.0(4.8)$ \\
\hline $\begin{array}{l}\text { Disease duration } \\
\text { in years }\end{array}$ & $\begin{array}{c}119 \\
(83.2 \%)\end{array}$ & $\begin{array}{c}33444 \\
(75.4 \%)\end{array}$ & $15.9(5.9)$ & $\begin{array}{c}50 \\
(89.3 \%)\end{array}$ & $\begin{array}{c}19612 \\
(71.0 \%)\end{array}$ & $16.7(6.2)$ & $\begin{array}{c}60 \\
(87.0 \%)\end{array}$ & $\begin{array}{c}23016 \\
(71.2 \%)\end{array}$ & $16.7(6.3)$ \\
\hline$\%$ Males & $\begin{array}{c}134 \\
(93.7 \%)\end{array}$ & $\begin{array}{c}35767 \\
(80.6 \%)\end{array}$ & $75.5(8.5)$ & $\begin{array}{c}53 \\
(94.6 \%)\end{array}$ & $\begin{array}{c}20821 \\
(75.4 \%)\end{array}$ & $73.0(7.3)$ & $\begin{array}{c}65 \\
(94.2 \%)\end{array}$ & $\begin{array}{c}25488 \\
(78.8 \%)\end{array}$ & $73.3(7.5)$ \\
\hline$\%$ HLA-B27+ & $\begin{array}{c}105 \\
(73.4 \%)\end{array}$ & $\begin{array}{c}27327 \\
(61.6 \%)\end{array}$ & $\begin{array}{c}84.0 \\
(11.3)\end{array}$ & $\begin{array}{c}39 \\
(69.6 \%)\end{array}$ & $\begin{array}{c}16231 \\
(58.8 \%)\end{array}$ & $79.9(11.0)$ & $\begin{array}{c}43 \\
(62.3 \%)\end{array}$ & $\begin{array}{c}18052 \\
(55.8 \%)\end{array}$ & $\begin{array}{c}81.2 \\
(10.4)\end{array}$ \\
\hline \multicolumn{10}{|c|}{ Geographic area of study: } \\
\hline - Europe & $\begin{array}{c}65 \\
(45.5 \%)\end{array}$ & $\begin{array}{c}25006 \\
(56.4 \%)\end{array}$ & - & $\begin{array}{c}35 \\
(62.5 \%)\end{array}$ & $\begin{array}{c}21740 \\
(78.7 \%)\end{array}$ & - & $\begin{array}{c}39 \\
(56.5 \%)\end{array}$ & $\begin{array}{c}20123 \\
(62.2 \%)\end{array}$ & - \\
\hline - North- America & $14(9.8 \%)$ & $\begin{array}{c}2901 \\
(6.5 \%)\end{array}$ & - & $\begin{array}{c}10 \\
(17.9 \%)\end{array}$ & $\begin{array}{c}2007 \\
(7.3 \%)\end{array}$ & - & $\begin{array}{c}10 \\
(14.5 \%)\end{array}$ & $\begin{array}{c}2866 \\
(8.9 \%)\end{array}$ & - \\
\hline - Latin-America & $11(7.7 \%)$ & $\begin{array}{c}1358 \\
(3.1 \%)\end{array}$ & - & $1(1.8 \%)$ & $58(0.2 \%)$ & - & $3(4.3 \%)$ & $380(1.2 \%)$ & - \\
\hline -Asia & $\begin{array}{c}34 \\
(23.8 \%)\end{array}$ & $\begin{array}{c}9923 \\
(22.4 \%)\end{array}$ & - & $2(3.6 \%)$ & $\begin{array}{c}2057 \\
(7.4 \%)\end{array}$ & - & $6(8.7 \%)$ & $\begin{array}{c}3331 \\
(10.3 \%)\end{array}$ & - \\
\hline - Middle-East & $14(9.8 \%)$ & $\begin{array}{c}1502 \\
(3.4 \%)\end{array}$ & - & $4(7.1 \%)$ & $537(1.9 \%)$ & - & $5(7.2 \%)$ & $503(1.6 \%)$ & - \\
\hline - Australia & $1(0.7 \%)$ & $74(0.2 \%)$ & - & 0 & 0 & - & 0 & 0 & - \\
\hline - Several areas & $4(2.8 \%)$ & $\begin{array}{l}3608 \\
(8.1 \%)\end{array}$ & - & $4(7.1 \%)$ & $\begin{array}{l}1227 \\
(4.4 \%)\end{array}$ & - & $6(8.7 \%)$ & $\begin{array}{c}5138 \\
(15.9 \%)\end{array}$ & - \\
\hline
\end{tabular}

$\mathrm{AAU}=$ acute anterior uveitis; $\mathrm{IBD}=$ inflammatory bowel disease, HLA-B27 = Human Leukocyte Antigen-B27. "Number of studies or number of patients (\% of total number of studies or patients) for which data of the specific variable was available

$\ddagger$ The mean is weighted by the number of included persons in every single study which report the variable.

the included studies is shown in table 1 . The prevalence of AAU in patients with AS was reported in 143 articles ( 85 cross-sectional, 9 prospective, 28 retrospective, 20 intervention and 1 case-control study) and included 44372 patients of which 13071 (29.5\%) patients had a history of AAU. The prevalence of psoriasis was reported in 56 articles (28 cross-sectional, 5 prospective, 9 retrospective, 12 intervention and 2 case-control studies) and included 27626 patients, of which 2980 (10.8\%) patients had (a history of) psoriasis. The prevalence of IBD was reported in 69 articles (38 cross-sectional, 7 prospective, 10 retrospective, 12 intervention and 2 case-control studies) and included 32341 patients, of which 2251 (7.0\%) patients had (a history of) IBD. 


\section{Risk of bias}

Risk of bias was assessed in all 156 studies. A risk of bias summary for all included studies is shown in online supplementary table 2 and a risk of bias graph for each EAM in online supplementary figure 1 , respectively.

With respect to the external validity, the most frequently source of bias was found in the random selection of patients, for which in only $20 \%$ to $26 \%$ of the studies the risk of bias was considered low. The risk of bias due to the sampling frame or the case definition for AS was considered low in $78 \%$ to $87 \%$ and $58 \%$ to $79 \%$ of the studies, respectively. The response rate was reported in $20 \%$ to $30 \%$ of the studies only, and therefore, the nonresponse bias was difficult to assess. With respect to internal validity, the risk of bias of the case definition for the EAM was considered low in $43 \%$ to $51 \%$ of the studies.

\section{Meta-analysis and subgroup analysis}

AAU. The pooled prevalence of AAU was $25.8 \%$ (95\% CI 24.1-27.6) with a substantial heterogeneity observed $\left(\mathrm{Q}=2374.63, \mathrm{p}<0.01, \mathrm{I}^{2}=94.0 \%\right)$. Figure 2 shows the prevalence estimates of AAU according to the different clinical- and methodological characteristics. The prevalence of AAU was significantly associated with disease duration and increased from $17.4 \%$ (95\% Cl 14.3-21.3) in studies including patients with a mean disease duration of $<10$ years to $38.5 \%(95 \% \mathrm{Cl} 33.5-43.9)$ in those with a mean disease duration of $>20$ years. Furthermore, the prevalence was significantly different across geographic regions. Prevalence estimates were highest in studies from North-America (35.2\%) and Europe (29.3\%), and lower in studies from Asia (21.4\%) and Latin America (20.1\%). The prevalence of AAU varied also significantly according to the case definition for the EAM, and was highest for self-report (35.9\%).

Finally, the prevalence was lower in studies considered to have a 'low risk of bias' $(20.8 \%$, $95 \% \mathrm{Cl} 16.9-25.3 \%)$ compared with studies with a high risk of bias $(26.7 \%, 95 \% \mathrm{Cl}$ 24.7-28.8).

Psoriasis. The pooled prevalence of psoriasis was $9.3 \%$ (95\% Cl 8.1-10.6) with substantial heterogeneity observed $\left(\mathrm{Q}=608.17, \mathrm{p}<0.01, \mathrm{I}^{2}=91.0 \%\right)$. Figure 3 shows the prevalence of psoriasis according to the different clinical- and methodological characteristics.

The reported prevalence of psoriasis varied significantly between different geographic areas, with the highest prevalence in studies from Europe (10.9\%) and the lowest in studies from Asia (3.1\%) and the Middle-East (4.2\%).

IBD. The pooled prevalence of IBD was $6.8 \%(95 \% \mathrm{Cl} 6.1-7.7)$ with a substantial heterogeneity observed $\left(\mathrm{O}=422.80, \mathrm{p}<0.01, \mathrm{I}^{2}=84.2 \%\right)$. Figure 4 shows the prevalence of IBD according to the different clinical and methodological characteristics. Only geographic area was associated with heterogeneity in the subgroup analysis $(p=0.03)$, with the highest prevalence in Latin America (9.6\%) and the lowest in studies from Asia (2.9\%). 


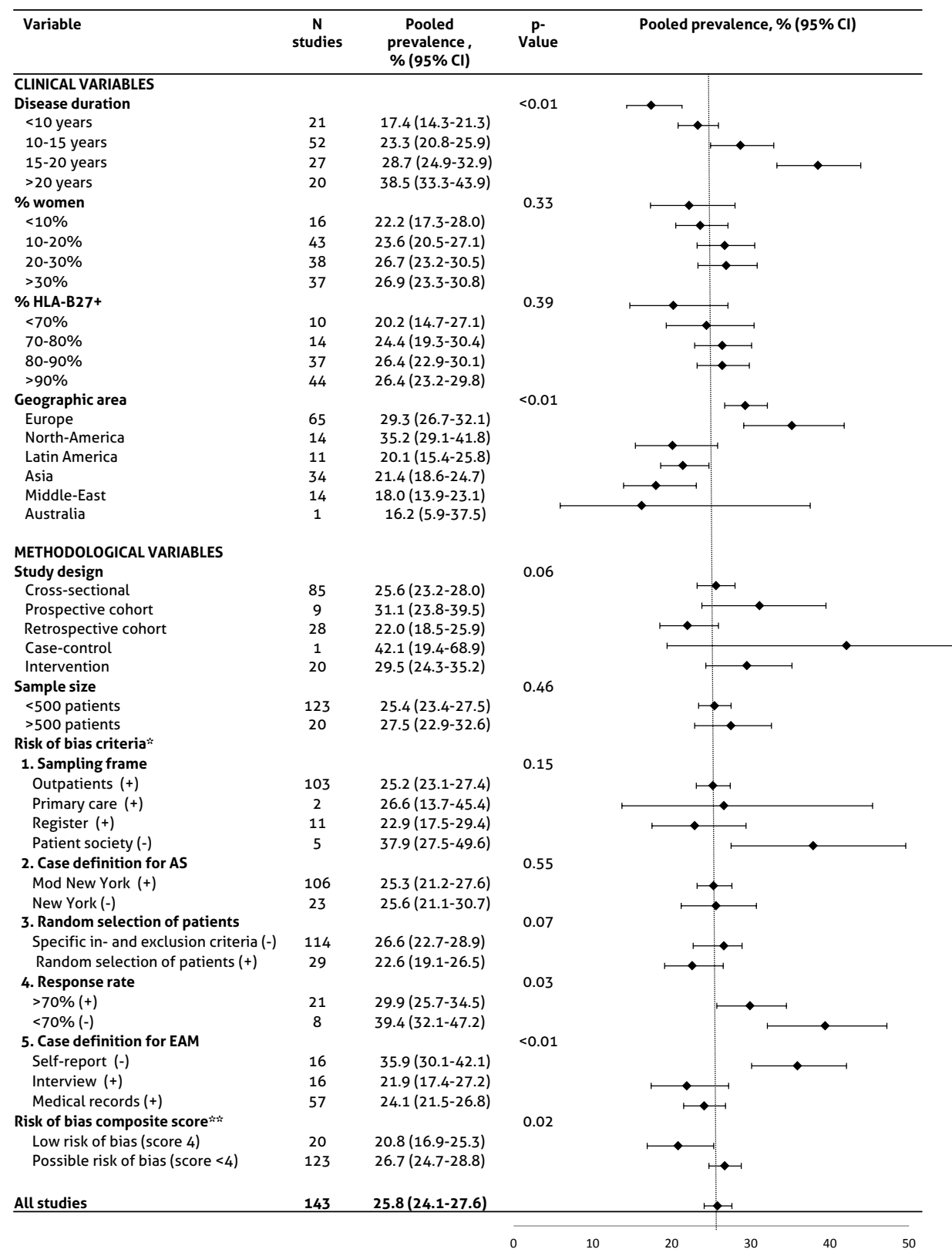

Figure 2 - Prevalence of AAU in patients with AS grouped by clinical- and methodological characteristics. EAM: extra-articular manifestation; $\mathrm{Cl}=$ confidence interval; * Individual risk of bias criteria, "+" considered as low risk of bias, "-" as high risk of bias **Sum of individual risk of bias scores, the item on response rate was not considered in the composite score because too many scores were not reported 


\begin{tabular}{|c|c|c|c|c|}
\hline Variable & $\begin{array}{c}\mathrm{N} \\
\text { studies }\end{array}$ & $\begin{array}{c}\text { Pooled } \\
\text { prevalence, } \\
\%(95 \% \mathrm{Cl})\end{array}$ & p-Value & Pooled prevalence, \% $(95 \% \mathrm{Cl})$ \\
\hline \multicolumn{5}{|l|}{ CLINICAL VARIABLES } \\
\hline Disease duration & & & 0.27 & \\
\hline$<10$ years & 5 & $7.7(4.6-12.8)$ & & $\leftrightarrow$ \\
\hline $10-15$ years & 20 & $10.1(7.9-12.8)$ & & 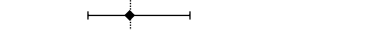 \\
\hline $15-20$ years & 11 & $9.1(6.4-13.0)$ & & $\bullet$ \\
\hline$>20$ years & 4 & $10.3(7.7-13.8)$ & & \\
\hline$\%$ women & & & 0.53 & - \\
\hline$<10 \%$ & 1 & $11.0(3.3-30.8)$ & & $\longmapsto$ \\
\hline $10-20 \%$ & 11 & $8.1(5.7-14.6)$ & & 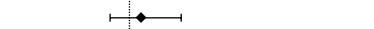 \\
\hline $20-30 \%$ & 22 & $9.9(7.9-12.5)$ & & $\longmapsto$ \\
\hline$>30 \%$ & 19 & $7.8(6.0-10.1)$ & & \\
\hline$\%$ HLA-B27+ & & & 0.75 & \\
\hline$<70 \%$ & 3 & $7.9(4.0-14.8)$ & & $\longrightarrow$ \\
\hline $70-80 \%$ & 8 & $8.9(6.2-12.7)$ & & $\longrightarrow$ \\
\hline $80-90 \%$ & 19 & $10.4(8.2-13.0)$ & & $\longrightarrow$ \\
\hline$>90 \%$ & 9 & $8.8(6.1-12.4)$ & & \\
\hline Geographic area & & & $<0.01$ & - \\
\hline Europe & 35 & $10.9(9.3-12.8)$ & & $\longmapsto$ \\
\hline North-America & 10 & $7.3(5.2-10.3)$ & & \\
\hline Latin America & 1 & $10.3(3.1-29.0)$ & & $\longmapsto$ \\
\hline Asia & 2 & $3.1(1.5-6.4)$ & & $\longmapsto$ \\
\hline Middle-East & 4 & $4.2(2.2-8.0)$ & & \\
\hline \multicolumn{5}{|l|}{ METHODOLOGICAL VARIABLES } \\
\hline Study design & & & 0.10 & \\
\hline Cross-sectional & 28 & $7.7(6.3-9.4)$ & & \\
\hline Prospective cohort & 5 & $9.8(6.0-15.4)$ & & \\
\hline Retrospective cohort & 9 & $11.6(8.4-16.0)$ & & \\
\hline Case-control & 2 & $7.8(3.8-15.4)$ & & \\
\hline Intervention & 12 & $11.8(8.5-16.1)$ & & \\
\hline Sample size & & & 0.82 & \\
\hline$<500$ patients & 40 & $9.3(7.7-11.2)$ & & \\
\hline$>500$ patients & 16 & $8.9(6.9-11.6)$ & & $\longmapsto$ \\
\hline \multicolumn{5}{|l|}{ Risk of bias criteria* } \\
\hline 1. Sampling frame & & & 0.35 & \\
\hline Outpatients (+) & 34 & $8.8(7.3-10.7)$ & & $\longmapsto$ \\
\hline Register $(+)$ & 12 & $8.2(6.0-11.2)$ & & $\longmapsto$ \\
\hline Patient society (-) & 4 & $12.8(7.9-20.3)$ & & \\
\hline 2. Case definition for AS & & & 0.61 & \\
\hline Mod New York (+) & 44 & $8.8(7.4-10.4)$ & & $\mapsto$ \\
\hline New York (-) & 5 & $10.8(7.0-15.2)$ & & $\rightarrow$ \\
\hline 3. Random selection of patients & & & 0.53 & \\
\hline Specific in- and exclusion criteria (-) & 41 & $9.4(7.9-11.3)$ & & $\longrightarrow$ \\
\hline Random selection of patients (+) & 15 & $8.5(6.3-11.3)$ & & 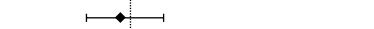 \\
\hline 4. Response rate & & & 0.32 & \\
\hline$\geq 70 \%(+)$ & 13 & $8.9(6.4-12.2)$ & & $\longmapsto$ \\
\hline$<70 \%(-)$ & 3 & $12.6(6.8-22.7)$ & & \\
\hline 5. Case definition for EAM & & & 0.90 & \\
\hline Self-report (-) & 8 & $8.8(6.0-12.5)$ & & $\longmapsto$ \\
\hline Interview (+) & 4 & $9.2(5.5-14.8)$ & & 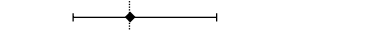 \\
\hline Medical records (+) & 19 & $8.9(6.9-11.5)$ & & 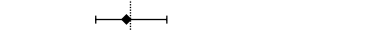 \\
\hline Risk of bias composite score & & & 0.18 & \\
\hline Low risk of bias (score 4) & 9 & $7.2(4.9-10.6)$ & & $\longmapsto$ \\
\hline Possible risk of bias (score <4) & 47 & $9.6(8.1-11.3)$ & & $\mapsto$ \\
\hline All studies & 56 & $9.3(8.1-10.6)$ & & $\mapsto$ \\
\hline
\end{tabular}

Figure 3 - Prevalence of psoriasis in patients with AS grouped by clinical- and methodological characteristics. EAM: extra-articular manifestation; $\mathrm{Cl}=$ confidence interval; * Individual risk of bias criteria, "+" considered as low risk of bias, "-" as high risk of bias **Sum of individual risk of bias scores, the item on response rate was not considered in the composite score because too many scores were not reported 


\begin{tabular}{|c|c|c|c|c|}
\hline & $\begin{array}{c}\mathrm{N} \\
\text { studies }\end{array}$ & $\begin{array}{c}\text { Pooled } \\
\text { prevalence \%, } \\
(95 \% \mathrm{Cl})\end{array}$ & P-Value & Pooled prevalence, $\%(95 \% \mathrm{Cl})$ \\
\hline \multicolumn{5}{|l|}{ CLINICAL VARIABLES } \\
\hline Disease duration & & & 0.57 & \\
\hline$<10$ years & 6 & $6.5(4.0-10.4)$ & & $\longrightarrow$ \\
\hline $10-15$ years & 28 & $6.0(4.9-7.4)$ & & $\mapsto$ \\
\hline $15-20$ years & 11 & $7.1(5.0-9.9)$ & & $\longrightarrow$ \\
\hline$>20$ years & 15 & $7.0(5.0-9.8)$ & & $\longmapsto$ \\
\hline$\%$ women & & & 0.17 & \\
\hline$<10 \%$ & 2 & $7.0(3.4-13.9)$ & & 5 \\
\hline $10-20 \%$ & 13 & $4.9(3.6-6.8)$ & & $\mapsto$ \\
\hline $20-30 \%$ & 26 & $6.6(5.7-8.1)$ & & $\mapsto$ \\
\hline$>30 \%$ & 24 & $7.7(6.2-9.6)$ & & $\longrightarrow$ \\
\hline$\%$ HLA-B27+ & & & 0.68 & \\
\hline$<70 \%$ & 3 & $7.9(4.0-15.1)$ & & $\longmapsto$ \\
\hline $70-80 \%$ & 7 & $5.7(3.7-8.6)$ & & $\longmapsto$ \\
\hline $80-90 \%$ & 22 & $7.4(5.8-9.4)$ & & 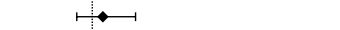 \\
\hline$>90 \%$ & 11 & $6.4(4.5-9.0)$ & & $\longmapsto$ \\
\hline Geographic area & & & 0.03 & \\
\hline Europe & 39 & $7.2(6.1-8.5)$ & & -1 \\
\hline North-America & 10 & $7.6(5.5-10.4)$ & & $\vdash$ \\
\hline Latin America & 3 & $9.6(5.4-16.9)$ & & 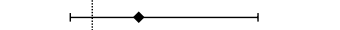 \\
\hline Asia & 6 & $2.9(1.9-4.4)$ & & $\mapsto$ \\
\hline Middle-East & 5 & $7.0(4.0-12.0)$ & & $\longmapsto$ \\
\hline \multicolumn{5}{|l|}{ METHODOLOGICAL VARIABLES } \\
\hline Study design & & & 0.72 & \\
\hline Cross-sectional & 38 & $6.3(5.2-7.5)$ & & $\mapsto-$ \\
\hline Prospective cohort & 7 & $7.9(5.3-11.6)$ & & $\longmapsto \longmapsto$ \\
\hline Retrospective cohort & 10 & $7.4(5.3-10.3)$ & & $\longmapsto$ \\
\hline Case-control & 2 & $8.8(4.3-17.0)$ & & 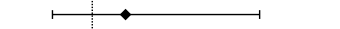 \\
\hline Intervention & 12 & $7.0(5.0-9.7)$ & & $\longrightarrow$ \\
\hline Sample size & & & 0.33 & \\
\hline$<500$ patients $(n=51)$ & 51 & $7.2(6.0-8.5)$ & & $\rightarrow$ \\
\hline$>500$ patients $(n=18)$ & 18 & $6.2(5.0-7.7)$ & & $\mapsto$ \\
\hline \multicolumn{5}{|l|}{ Risk of bias criteria" } \\
\hline 1. Sampling frame & & & 0.73 & \\
\hline Outpatients (+) & 43 & $6.6(5.6-7.9)$ & & $\mapsto-1$ \\
\hline Primary care $(+)$ & 2 & $9.9(4.8-20.6)$ & & $\longmapsto$ \\
\hline Register $(+)$ & 15 & $6.4(4.9-8.2)$ & & $\mapsto$ \\
\hline Patient society (-) & 3 & $7.7(4.5-13.0)$ & & $\bullet$ \\
\hline 2. Case definition for AS & & & 0.56 & \\
\hline Mod New York (+) & 54 & $6.8(5.8-7.9)$ & & $\mapsto$ \\
\hline New York (-) & 6 & $5.6(3.7-8.5)$ & & $\longmapsto$ \\
\hline 3. Random selection of patients & & & 0.70 & \\
\hline Specific in- and exclusion criteria (-) & 50 & $6.7(5.7-7.8)$ & & $\leftrightarrow$ \\
\hline Random selection of patients (+) & 18 & $7.1(5.5-9.1)$ & & $\mapsto$ \\
\hline 4. Response rate & & & 0.08 & \\
\hline$\geq 70 \%(+)$ & 11 & $6.5(5.4-7.8)$ & & $\mapsto-1$ \\
\hline$<70 \%(-)$ & 6 & $8.5(6.7-10.7)$ & & $\longmapsto$ \\
\hline 5. Case definition for EAM & & & 0.71 & \\
\hline Self-report (-) & 15 & $6.4(4.9-8.4)$ & & $\mapsto$ \\
\hline Interview (+) & 8 & $6.1(4.1-9.0)$ & & $\longmapsto$ \\
\hline Medical records (+) & 22 & $6.4(5.1-8.0)$ & & $\mapsto$ \\
\hline Screening $(+)$ & 2 & $6.7(2.4-17.6)$ & & \\
\hline Risk of bias composite score & & & 0.99 & \\
\hline Low risk of bias (score 4) & 12 & $6.8(4.9-9.3)$ & & $\longmapsto$ \\
\hline Possible risk of bias (score <4) & 57 & $6.8(5.9-7.9)$ & & $1+-1$ \\
\hline All studies & 69 & $6.8(6.1-7.7)$ & & $\Leftrightarrow-1$ \\
\hline
\end{tabular}

Figure 4 - Prevalence of inflammatory bowel disease in patients with AS grouped by clinicaland methodological characteristics. IBD: inflammatory bowel disease; EAM: extra-articular manifestation; $\mathrm{Cl}=$ confidence interval; " Individual risk of bias criteria, "+" considered as low risk of bias, "-" as high risk of bias "*Sum of individual risk of bias scores, the item on response rate was not considered in the composite score because too many scores were not reported 


\section{Meta-regression analysis}

Table 2 shows the results of the univariable and multivariable meta-regression analysis of clinical- and methodological characteristics exploring the heterogeneity of the reported prevalences. No interaction was found between the variables. All VIF statistics were well below the recommended cut-off value of 10 .

$A A U$. In the final multivariable meta-regression model on the prevalence of AAU in AS, the clinical characteristics disease duration $(\beta 0.05,95 \% \mathrm{Cl} 0.03 ; 0.06)$ and geographic area $(\beta-0.64,95 \% \mathrm{Cl}-1.00 ;-0.28$ in studies in the Middle-East compared with studies from Europe) remained significantly associated with the prevalence of AAU. Random selection of patients, was the only 'risk of bias' criterion which was significantly associated with prevalence of AAU $(\beta-0.24,95 \% \mathrm{Cl}-0.43 ;-0.04)$. The regression model explained $44.9 \%$ of the total heterogeneity.

Psoriasis. In the multivariable meta-regression model on the prevalence of psoriasis in AS, only geographic area was significantly associated with the prevalence of psoriasis, with lower prevalence estimates in studies from Asia $(\beta-1.33,95 \% \mathrm{Cl}-2.09 ;-0.57)$ and the Middle-East $(\beta-1.03,95 \% \mathrm{Cl}-1.73 ;-0.32)$ compared to studies from Europe. The regression model explained $27.2 \%$ of the total heterogeneity.

IBD. In the multivariable meta-regression model on the prevalence of IBD, geographic area (lower in studies from Asia $(\beta-0.80,95 \% \mathrm{Cl}-1.29$; -0.32$)$ compared to Europe) and the percentage of females included in the studies $(\beta 0.02,95 \% \mathrm{Cl} 0.00 ; 0.03)$ were significantly associated with the prevalence of IBD. The regression model explained $25.7 \%$ of the total heterogeneity.

\section{DISCUSSION}

The present review confirmed high prevalences of EAMs in patients with AS. The pooled prevalence of $A A U$ in patients with AS was $25.8 \%$, whereas a lifetime cumulative incidence of AAU in the general population is reported to be $0.2 \%$ in HLA-B27-negative and $1 \%$ in HLA-B27-positive subjects [17]. The pooled prevalence of psoriasis in AS was $9.3 \%$ and for IBD $6.8 \%$, both are considerably higher than general population estimates which vary from $0.3 \%$ to $2.5 \%$ for psoriasis and from $0.01 \%$ to $0.5 \%$ for IBD [18] [19]. This study is the first using a systematic approach with meta-analysis to estimate the prevalence of AAU, psoriasis and IBD in patients with AS. Zeboulon et al. performed an SLR on the prevalence of AAU and reported a (crude) prevalence of 33.2\% (mean disease duration of 17 years), which increased with disease duration and was higher in HLA-B27 positive patients. The prevalence is comparable to our study, although we found a lower prevalence $(20 \%)$, when limiting the analysis to high quality studies. While we confirmed a trend of higher prevalences in studies with a higher percentage of HLA-B27 positive 


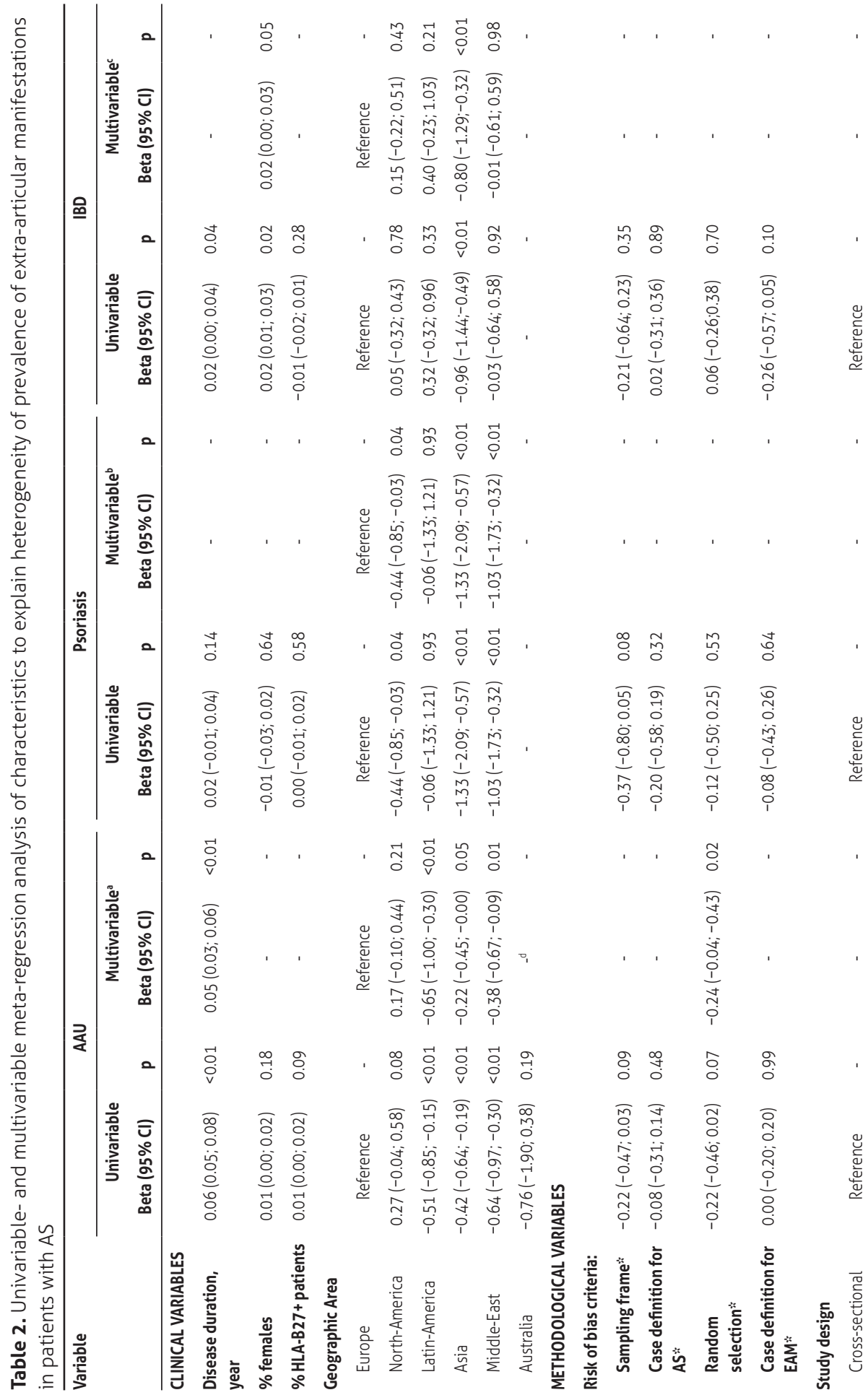




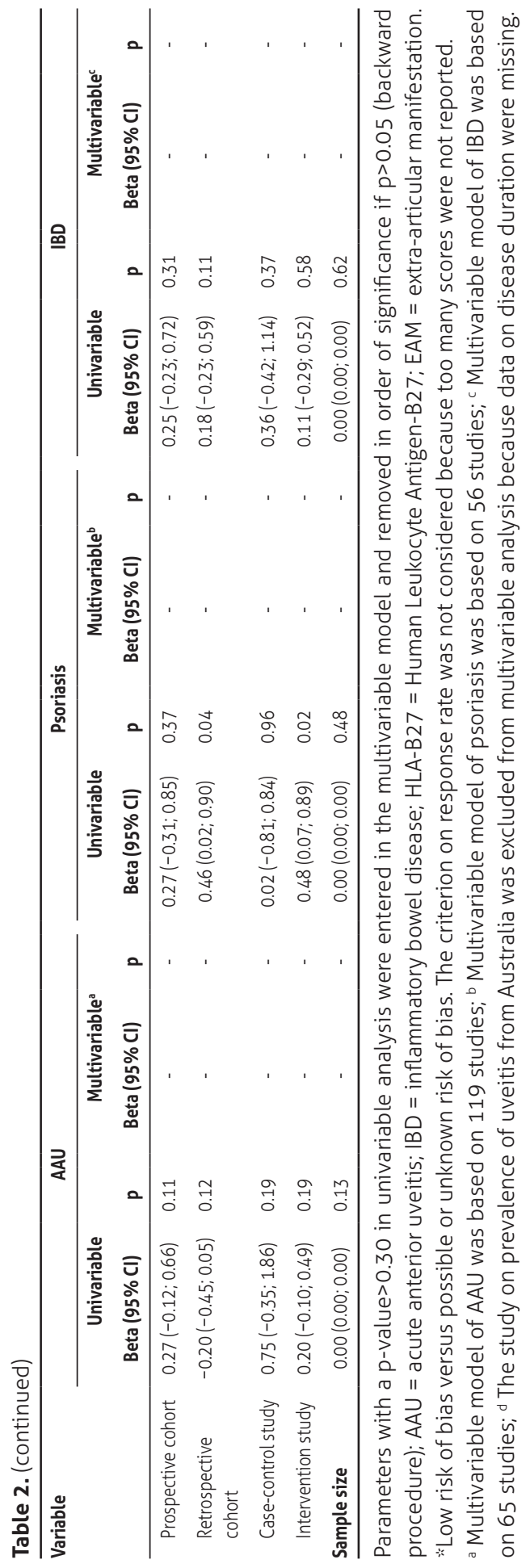


patients, this was not significant in our multivariable analyses. SLRs on the prevalence of psoriasis and IBD in AS are lacking. The present findings are in the lower range of the general estimates of 5 to $10 \%$ and 10 to $25 \%$ for IBD and psoriasis, respectively.

A meta-regression analysis was performed to explore the methodological and clinical heterogeneity among the studies. Disease duration and geographic area were the most striking contributors to heterogeneity found. Specifically, the prevalence of AAU was associated with disease duration, and ranged from $17.4 \%$ among studies in patients with a mean disease duration of $<10$ years to $38.5 \%$ among studies in patients with a mean disease duration of $>20$ years. This association with disease duration was neither found for psoriasis nor for IBD. This suggests, but does not prove, that psoriasis and IBD may already be present before the onset or before diagnosis of AS. It should be noted, however, that studies including patients with a short disease duration (<10 years) were underrepresented in the present review. Because the EAMs are now part of the classification criteria for axSpA [20] and moreover are considered as contributory to a diagnosis of axSpA [3], the relationship between both the onset of AS and onset of EAMs needs more attention in future studies.

Interestingly, variation in prevalence of EAMs was found across different geographic areas. Genetic factors may explain part of the variation. It is known that AAU is more prevalent in HLA-B27 positive patients compared with HLA-B27 negative patients [21]. As there is a geographic difference in the percentage of patients with AS who possess HLA-B27 [22] [23], it could be expected that part of the geographic variation in the prevalence of EAM is due to the prevalence of HLA-B27. In the present study, however, most differences between geographic areas remained significant when controlling for HLA-B27 in the multivariable regression, suggesting that other (epi)genetic factors, are likely to play a role. It should be reminded that the number of studies and patients included from Africa, Latin-America and Australia were limited, and that in meta-regressions associations with HLA-B27 and EAM are explored at the study level and not at the individual patient level. A lower risk of bias based on the composite score tended to provide lower estimates of the prevalence of AAU and psoriasis in univariable analyses. In the multivariable analysis, high risk of bias in random selection of patients contributed independently to higher prevalence rates of AAU. A possible explanation could be that patients in trials were selected based on higher disease activity and that this may coincide with a higher prevalence of AAU [24].

Some limitations of the present study should be recognized. First, the prevalence of EAMs used in this review was usually not the main objective of the primary studies included and therefore not always indexed in the electronic article databases. We applied a broad search strategy without any terms for 'prevalence' or 'epidemiology'. However, despite additional efforts of the authors, probably not all studies reporting EAMs in AS were captured with the search strategy. This was also emphasized by the high number of 
additional articles that were retrieved by 'snowballing'. Second, despite the fact that all studies were checked for double counting of the same cohort, we cannot fully exclude that sub-groups of patients participated in several studies and therefore were included twice (or more) in the assessment of the EAM. Third, a large heterogeneity was found and although this was explored in subgroup- and meta-regression analyses, these analyses have some limitations [14]. Results from meta-regressions are observational, and therefore can suffer from bias by confounding, which means that an association identified with one study characteristic may reflect a true association with other correlated characteristics, whether these are known or unknown. Moreover, in some studies, information was lacking on, for example, disease duration. Therefore, analyses could only be based on the studies with available information, potentially biasing the results. Further, patients' characteristics are based on group-averages. Such analyses are difficult to interpret, because the relationship on study level may not be the same as the relationship on patient level. Last, the variability between studies may be lower than the within-studies, which makes it sometimes more difficult to show significant results of true relationships.

In conclusion, this SLR with meta-analysis summarized the prevalence of AAU, psoriasis and IBD in patients with AS. Awareness among clinicians of EAMs is important in view of their role in the diagnostic process, for treatment choices and for health-related quality of life. The prevalence of EAMs among patients with AS is clearly increased compared with the general population. While AAU occurs clearly more frequently in patients with longer disease duration, this was less clear for IBD and psoriasis.

\section{SUPPLEMENTARY DATA}

Supplementary data are published on the website of the Annals of the Rheumatic Diseases. 


\section{REFERENCES}

1. Sieper J, Braun J, Rudwaleit M, et al. Ankylosing spondylitis: an overview. Ann Rheum Dis 2002;61:8-18.

2. Elewaut D, Matucci-Cerinic M. Treatment of ankylosing spondylitis and extra-articular manifestations in everyday rheumatology practice. Rheumatology (Oxford) 2009;48:1029-35.

3. Rudwaleit M, van der Heijde D, Khan MA, et al. How to diagnose axial spondyloarthritis early. Annals of the rheumatic diseases 2004;63:535-43.

4. de Groot V, Beckerman $\mathrm{H}$, Lankhorst G], et al. How to measure comorbidity. a critical review of available methods. J Clin Epidemiol 2003;56:221-9.

5. Brophy S, Pavy S, Lewis P, et al. Inflammatory eye, skin, and bowel disease in spondyloarthritis: genetic, phenotypic, and environmental factors. ] Rheumatol 2001:28:2667-73.

6. Chorus AM, Miedema HS, Boonen A, et al. Quality of life and work in patients with rheumatoid arthritis and ankylosing spondylitis of working age. Ann Rheum Dis 2003;62:1178-84.

7. Robertson LP, Davis MJ. A longitudinal study of disease activity and functional status in a hospital cohort of patients with ankylosing spondylitis. Rheumatology (Oxford) 2004;43:1565-8.

8. Baeten $D$, Breban M, Lories R, et al. Are spondylarthritides related but distinct conditions or a single disease with a heterogeneous phenotype? Arthritis Rheum 2013:65:12-20.

9. Zeboulon N, Dougados M, Gossec L. Prevalence and characteristics of uveitis in the spondyloarthropathies: a systematic literature review. Ann Rheum Dis 2008;67:955-9.

10. Shamliyan TA, Kane RL, Ansari MT, et al. Development quality criteria to evaluate nontherapeutic studies of incidence, prevalence, or risk factors of chronic diseases: pilot study of new checklists. ] Clin Epidemiol 2011;64:637-57.

11. Higgins JP, Thompson SG. Quantifying heterogeneity in a meta-analysis. Stat Med 2002;21:1539-58.

12. Higgins JP, Thompson SG, Deeks J], et al. Measuring inconsistency in meta-analyses. Bmj 2003;327: 557-60.

13. Lipsey M, Wilson D. Practical meta-analysis. California: Sage publications; 2001.

14. Thompson SG, Higgins JP. How should meta-regression analyses be undertaken and interpreted? Stat Med 2002;21:1559-73.

15. Myers RH. Classical and modern regression with Applications. Boston, MA: PWS-Kent; 1990.

16. Wilson D. METAREG for SPSS/Win 6.1 or higher MACRO. 2001; Available from: http://mason.gmu. edu/ dwilsonb/ma.html.

17. Linssen A, Rothova A, Valkenburg HA, et al. The lifetime cumulative incidence of acute anterior uveitis in a normal population and its relation to ankylosing spondylitis and histocompatibility antigen HLA-B27. Invest Ophthalmol Vis Sci 1991;32:2568-78.

18. Plunkett A, Marks R. A review of the epidemiology of psoriasis vulgaris in the community. Australas ] Dermatol 1998:39:225-32.

19. Molodecky NA, Soon IS, Rabi DM, et al. Increasing incidence and prevalence of the inflammatory bowel diseases with time, based on systematic review. Gastroenterology 2012;142:46-54 e42; quiz e30.

20. Rudwaleit M, van der Heijde D, Landewe R, et al. The development of Assessment of SpondyloArthritis international Society classification criteria for axial spondyloarthritis (part II): validation and final selection. Ann Rheum Dis 2009;68:777-83.

21. Feldtkeller $E$, Khan MA, van der Heijde $D$, et al. Age at disease onset and diagnosis delay in HLA-B27 negative vs. positive patients with ankylosing spondylitis. Rheumatol Int 2003;23:61-6.

22. Stolwijk C, Boonen A, van Tubergen A, et al. Epidemiology of spondyloarthritis. Rheum Dis Clin North Am 2012;38:441-76.

23. Abdelrahman MH, Mahdy S, Khanjar IA, et al. Prevalence of HLA-B27 in Patients with Ankylosing Spondylitis in Qatar. Int ] Rheumatol 2012;2012:860213.

24. Chen $\mathrm{CH}$, Lin KC, Chen HA, et al. Association of acute anterior uveitis with disease activity, functional ability and physical mobility in patients with ankylosing spondylitis: a cross-sectional study of Chinese patients in Taiwan. Clinical rheumatology 2007;26:953-7. 



\section{CHAPTER 4}

\section{The epidemiology of extra-articular manifestations in ankylosing spondylitis: a population-based matched cohort study}

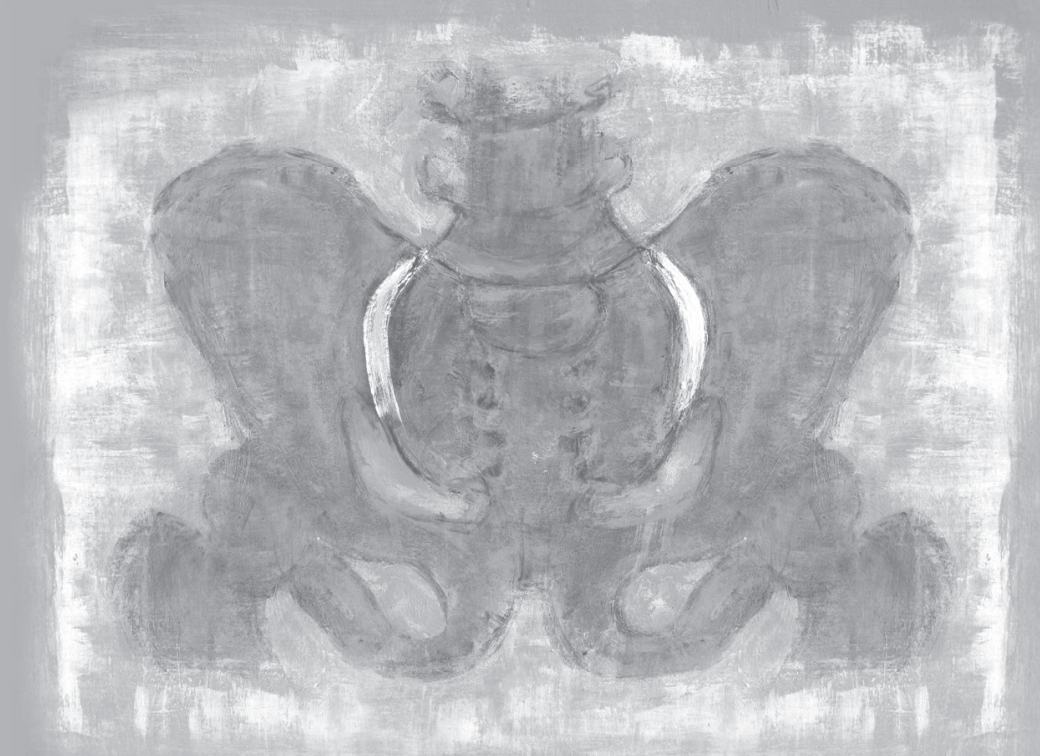

Carmen Stolwijk, Ivette Essers, Astrid van Tubergen, Annelies Boonen, Marloes T Bazelier, Marie L. De Bruin, Frank de Vries 


\section{ABSTRACT}

\section{Objective}

To assess the incidence and risks of common extra-articular manifestations (EAMs), i.e. acute anterior uveitis (AAU), psoriasis and inflammatory bowel disease (IBD), in patients with ankylosing spondylitis (AS) compared with population-based controls.

\section{Methods}

All incident patients with AS $(n=4,101)$ from the UK Clinical Practice Research Datalink (1987-2012) were matched with up to seven control subjects without AS by year of birth, sex and practice $(n=28,591)$. Incidence rates, cumulative incidence rates and adjusted (adj.) hazard ratios (HR) for the development of EAMs were calculated, with time-dependent adjustments for age, sex, comorbidity and medication use.

\section{Results}

At diagnosis of AS, the proportion of patients with an EAM was $11.4 \%$ for AAU, $4.4 \%$ for psoriasis and $3.7 \%$ for IBD. Incidence rates of EAMs were 8.9/1,000 person-years for $A A U, 3.4 / 1,000$ person-years for psoriasis and 2.4/1,000 person-years for IBD in AS. The 20-year cumulative incidence was $24.5 \%, 10.1 \%$ and $7.5 \%$, respectively. Risks of EAMs were 1.5- to 16-fold increased versus controls, with an adj. HR of 15.5 (95\% $\mathrm{Cl}, 11.6-20.7)$ for $\mathrm{AAU}$, adj. HR of 1.5 (95\% Cl, 1.1-1.9) for psoriasis and adj. HR of 3.3 (95\% Cl, 2.3-4.8) for IBD. For psoriasis and IBD, the highest risks were found in the first years after diagnosis, while developing AAU continued to be increased also 10 years after diagnosis of AS.

\section{Conclusion}

The risk of, in particular AAU, but also of psoriasis and IBD, is significantly increased in patients with AS compared with controls. Hazard patterns are different for each of the EAMs. 
The prevalence of EAMs: a cohort study $\mid 63$

\section{INTRODUCTION}

Ankylosing spondylitis (AS) is a chronic, inflammatory rheumatic disease with an estimated incidence of 3 to 7 per 100,000 person-years and an estimated prevalence up to $0.6 \%$ in Western populations [1-4]. AS is the prototype of a group of rheumatic diseases referred to as spondyloarthritis (SpA), which share genetic, clinical and radiographic features. Although AS is characterized by axial and peripheral joint manifestations, multiple other organ systems can be involved during the disease course [5]. Already during the 1960s, Moll and Wright observed the striking association between AS and several other disorders, such as psoriasis and inflammatory bowel disease (IBD) [6]. Nowadays, acute anterior uveitis (AAU), psoriasis, and IBD are considered as the three common extraarticular manifestations (EAMs) of AS, which are related to the concept of SpA $[5,7]$.

EAMs are of growing interest because of their role in the diagnosis of SpA and their impact on a patient's health related quality of life, as well as on treatment choices. The presence of one of the three concept-related EAMs, in particular AAU, increases the probability of axial or peripheral SPA in patients presenting with chronic back pain or peripheral arthritis. $[8,9]$ This is underlined by the inclusion of the EAMs in different criteria sets which aim to classify the whole spectrum of SpA, such as the Amor criteria and the European Spondyloarthropathy Study Group (ESSG) criteria [10, 11]. The EAMs are also inherently part of the recently developed Assessment of SpondyloArthritis international Society (ASAS) classification criteria for axial and peripheral SpA [12, 13]. Further, EAMs can add complexity to patient care, since their presence influences treatment decisions and may require collaboration with other specialists [14]. Moreover, EAMs can affect the prognosis and outcome of AS, especially health-related quality of life, work participation and health care costs, at any moment, and their presence should therefore be taken into account when studying health outcomes. EAMs are rather frequent in patients with AS and may present before or after the diagnosis of AS [7]. It has been estimated that AAU occurs in 20-30\% of patients with AS, psoriasis in 10-15\%, and IBD in $5-10 \%$ of patients $[7,15,16]$. However, these estimations are only based on cross-sectional data in selected populations.

Given the high prevalence of EAMs in patients with AS and their impact on diagnosis, treatment, prognosis and outcomes, it is relevant to gain insight in the epidemiology of the EAMs. To our knowledge, no longitudinal data on the relation between AS and the development of EAMs have been published. Moreover, studies comparing the frequency of occurrence of EAMs between patients with AS and population-based controls are scarce, and did not statistically adjust for a wide range of potential confounders [17]. The aims of the present study were 1 ) to determine the incidence rates and relative risks of $\mathrm{AAU}$, psoriasis, and IBD in patients with AS as compared with population-based controls, thereby taking into account potential confounders including comorbidities and drug use, and 2) to describe the timing of onset and hazard patterns of EAMs along the course of AS. 
64 Chapter 4

\section{METHODS}

\section{Data source}

A retrospective cohort study was conducted using data from the Clinical Practice Research Datalink (CPRD), formerly known as the General Practice Research Database (GPRD). The CPRD comprises prospectively collected computerized medical records for over 10 million patients under care of general practitioners (GP) from 1987 with ongoing data collection. Patients enrolled in CPRD are representative of the total United Kingdom (UK) population. The data recorded in the CPRD include patient demographics, lifestyle parameters, medical history, laboratory test results, referrals to consultants, hospitalizations and prescriptions. The accuracy and completeness of a wide range of diseases has been well validated and documented [18].

\section{Study population}

The study population (1987-2012) consisted of all patients aged 16 years or older with a first ever recording of AS during their period of valid data collection. The start of valid data collection of each practice was defined as the date at which the practice was included into CPRD, the GP's data set was approved as 'up-to-standard' and the practice was computerized. Each patient with AS was matched by year of birth, sex, calendar time and practice to up to 7 control subjects without a diagnosis of AS at any time. The date of the first AS diagnosis after valid data collection defined the index date. Control patients were assigned the same index date as their matched patient with AS. In three sub-cohorts for each EAM, every patient was followed from his index date (start of follow-up) until either the first occurrence of the EAM-outcome of interest or until the end of CPRD follow-up (i.e. the end of valid data collection, the date of the patient's transfer out of the practice or the patient's death).

\section{Study outcomes and confounding}

Outcomes of interest included the first ever event of AAU, psoriasis, or IBD (Crohn's disease or Ulcerative Colitis) after start of follow-up. Diagnoses of EAMs were identified by Read-codes (operational definitions are available upon request). Follow-up time was divided into 30-day intervals. Only incident outcomes of interest were evaluated, which means the three sub-cohorts only included patients and controls who did not have a history of the EAM-outcome of interest before index date.

The presence of potential confounders was assessed by reviewing the computerized medical records for any evidence of confounders before the start of an interval. Potential confounders that were determined for all EAMs included sex, body mass index (BMI), smoking status and alcohol use (all at index date), age, prescriptions for non-steroidal 
anti-inflammatory drugs (NSAIDs) in the 6 months before the start of an interval and the number of GP visits in the year before the start of an interval.

For each EAM, specific potential confounders were selected based on literature of potential risk factors for the development of the EAMs, including a history of (chronic) diseases, infections in the 6 months before the start of an interval and medication use in the 6 months before the start of an interval. Detailed information on the potential confounders for each EAM is shown in online supplementary text 1.

\section{Statistical analysis}

Differences in baseline characteristics between patients with AS and controls in dichotomous data were compared using chi-square testing. Incidence rates (and 95\% confidence interval $[\mathrm{Cl}]$ ) for each EAM were estimated as the number of patients, respectively controls, with the respective EAM per 1,000 person-years. Analyses were stratified for sex and age categories (16-29, 30-39, 40-49, 50-59 and $\geq 60$ years). Incidence rate ratios (IRR) (and 95\% CI) were calculated by dividing the incidence rate for patients by the incidence rate for controls. Nonparametric Kaplan-Meier methods were used to estimate the cumulative incidences (and $95 \% \mathrm{Cl}$ ) of the EAMs, which included both the presence of EAMs at index date and after index date.

Time-dependent Cox proportional hazards models were used to estimate hazard ratios (HR) for the risk of developing a new EAM after the index date in patients with AS versus controls. Analyses were stratified for sex, age at index date, and duration of disease (defined as the time since index date). Statistical time-dependent adjustments were made for all potential confounders that resulted in a change of the beta-coefficient $>1 \%$ in age/sex adjusted (adj.) analyses.

In our study, the date of diagnosis of AS was defined as the first ever recorded diagnosis of AS after valid data collection. However, information about the actual diagnosis may have lacked. In order to increase the likelihood of capturing true incident patients with AS, two sensitivity analyses were performed. First, we included only patients with AS whose first ever diagnosis had occurred at least 1 year after start of valid data collection. In the second sensitivity analysis, we stratified patients by their age at index date ( $<50$ years versus $\geq 50$ years), because usually AS is diagnosed before the age of 50 [19]. Therefore, patients aged $\geq 50$ years at index date are less likely true incident patients. All statistical analyses were conducted using SAS 9.1 software. 


\section{RESULTS}

\section{Baseline}

Baseline characteristics of the patients with AS $(n=4,101)$ and matched controls ( $n=28,591 ; 98 \%$ of patients having 7 controls) are presented in Table 1 . The mean age at index date was 43.7 years for AS patients and 43.6 years for controls, and $70.6 \%$ of

Table 1. Characteristics of patients with AS and matched controls at index date

\begin{tabular}{|c|c|c|}
\hline Characteristic & $\begin{array}{l}\text { Patients with AS (\%) } \\
\qquad N=4,101\end{array}$ & $\begin{array}{c}\text { Controls (\%) } \\
N=28,591\end{array}$ \\
\hline Male & $2,897(70.6)$ & $20,173(70.6)$ \\
\hline \multicolumn{3}{|c|}{ Age at index date } \\
\hline $16-29$ & $773(18.8)$ & $5,407(18.9)$ \\
\hline $30-39$ & $1,115(27.2)$ & $7,781(27.2)$ \\
\hline $40-49$ & $887(21.6)$ & $6,203(21.7)$ \\
\hline $50-59$ & $618(15.1)$ & $4,314(15.1)$ \\
\hline $60+$ & $708(17.3)$ & $4,886(17.1)$ \\
\hline \multicolumn{3}{|l|}{ Smoking } \\
\hline Current & $1,489(36.3)$ & $9,022(31.6) *$ \\
\hline Ex & $616(15.0)$ & $3,966(13.9)^{*}$ \\
\hline Never & $1,825(44.5)$ & $13,081(45.5)$ \\
\hline Unknown & $171(4.2)$ & $2,522(8.8)^{*}$ \\
\hline \multicolumn{3}{|l|}{ Alcohol } \\
\hline Yes & $2,817(68.7)$ & $19,118(66.9) *$ \\
\hline No & $647(15.8)$ & $35,88(12.5)^{* *}$ \\
\hline Unknown & $637(15.5)$ & $5,885(20.6) *$ \\
\hline \multicolumn{3}{|l|}{ BMI } \\
\hline$<20$ & $220(5.4)$ & $1,386(4.8)$ \\
\hline $20-25$ & $1,123(27.4)$ & $7,612(26.6)$ \\
\hline $25-30$ & $939(22.9)$ & $6,664(23.3)$ \\
\hline$>30$ & $500(12.2)$ & $3,409(11.9)$ \\
\hline Unknown & $1,319(32.2)$ & $9,520(33.3)$ \\
\hline \multicolumn{3}{|c|}{ Medication 6 months before index date } \\
\hline NSAID & $1,923(46.9)$ & $2,460(8.6)^{*}$ \\
\hline \multicolumn{3}{|c|}{ History of EAM before index date } \\
\hline AAU & $466(11.4)$ & $143(0.5) *$ \\
\hline Psoriasis & $182(4.4)$ & $749(2.6) *$ \\
\hline IBD & $151(3.7)$ & $176(0.6)^{* *}$ \\
\hline
\end{tabular}

* Statistically significant difference $(p<0.05)$ between patients with AS and controls, based on chisquare test.

$\mathrm{AS}=$ ankylosing spondylitis; $\mathrm{BMI}=$ body mass index; NSAID=non-steroidal anti-inflammatory drug; $E A M=e x t r a-a r t i c u l a r$ manifestation; $A A U=$ acute anterior uveitis; $I B D=$ inflammatory bowel disease 
the patients were male. The median duration of follow-up was 5.4 years both for patients and controls. Patients with AS were 5 times more likely to have recently been prescribed NSAIDs, compared with controls. Baseline characteristics of the three-sub-cohorts including patients and controls who did not have a diagnosis of AAU, psoriasis or IBD before index date, are shown in online supplementary table 1.

\section{Incidence rate, incidence rate ratios and cumulative incidence of EAMs}

Table 2 shows incidence rates as well as IRRs of the EAMs. Incidence rates of EAMs were 2 to 20 -fold increased with AS versus controls: IRR 21.1 (95\% CI 16.3-27.3) for AAU, 1.9 (95\% Cl 1.5-2.4) for psoriasis and 5.3 (95\% Cl 3.8-7.4) for IBD. All IRRs were higher in men as compared with women. They decreased with older age for AAU and IBD, and remained stable with age for psoriasis (Figure 1). Figure 2 shows that a substantial proportion of the EAMs occurred before the index date. For AAU, the cumulative incidence was $11.9 \%(95 \% \mathrm{Cl} 10.9-12.9)$ in AS at index date, compared to $0.5 \%$ (95\% Cl, 0.4-0.6) in controls, and increased in patients to $24.5 \%(95 \% \mathrm{Cl} 20.6-28.5)$ after 20 years, which was significantly faster than in controls. The cumulative incidence of psoriasis was $4.7 \%$ (95\% Cl 4.1-5.4) in patients compared to $2.6 \%$ (95\% Cl 2.4-2.8) in controls at the index date and increased, thereafter, gradually to $10.1 \%(95 \% \mathrm{Cl} 8.4-11.9)$ in patients after 20 years with a slope comparable with controls. The cumulative incidence of IBD in patients with AS showed a comparable pattern as psoriasis and increased from $4.0 \%(95 \% \mathrm{Cl}$ 3.4-4.6) at index date (versus $0.6 \%, 95 \% \mathrm{Cl} 0.5-0.7$ in controls) to $7.5 \%$ (95\% Cl 6.0-0.3) after 20 years.

Table 2. Incidence rate of AAU, psoriasis, and IBD in patients with AS and controls

\begin{tabular}{|c|c|c|c|c|c|}
\hline & \multicolumn{2}{|c|}{ Patients with AS } & \multicolumn{2}{|c|}{ Controls } & \multirow{2}{*}{$\begin{array}{l}\text { Incidence rate ratio } \\
\qquad(95 \% \mathrm{Cl})\end{array}$} \\
\hline & $\mathrm{EAM}, \mathrm{n}=$ & Incidence rate* & $\mathrm{EAM}, \mathrm{n}=$ & Incidence rate & \\
\hline AAU & 203 & 8.91 & 80 & 0.42 & $21.1(16.3-27.3)$ \\
\hline Psoriasis & 90 & 3.36 & 341 & 1.81 & $1.9(1.5-2.4)$ \\
\hline IBD & 62 & 2.36 & 84 & 0.44 & $5.3(3.8-7.4)$ \\
\hline
\end{tabular}

*Number of patients or controls with EAM/1,000 person-years, *** The incidence rate ratio is calculated as the incidence rate for patients divided by the incidence rate for controls $\mathrm{AAU}=$ acute anterior uveitis; $\mathrm{IBD}=$ inflammatory bowel disease; $\mathrm{AS}=$ ankylosing spondylitis; $\mathrm{Cl}=$ confidence interval

\section{Risk of EAMs with AS}

Table 3 shows that patients with AS had a 16-fold (adj. HR 15.5, 95\% Cl 11.6-20.7) increased risk of a first episode of AAU as compared with controls. The risk of psoriasis was 1.5-fold (adj. HR 1.5, 95\% Cl 1.1-1.9) and the risk of IBD was 3-fold (adj. HR 3.3, 95\% $\mathrm{Cl}$ 2.3-4.8) increased. Risks of EAMs were higher in men than in women. The risk of all EAMs was highest in the first year after diagnosis. While the risk of AAU was still 9-fold 


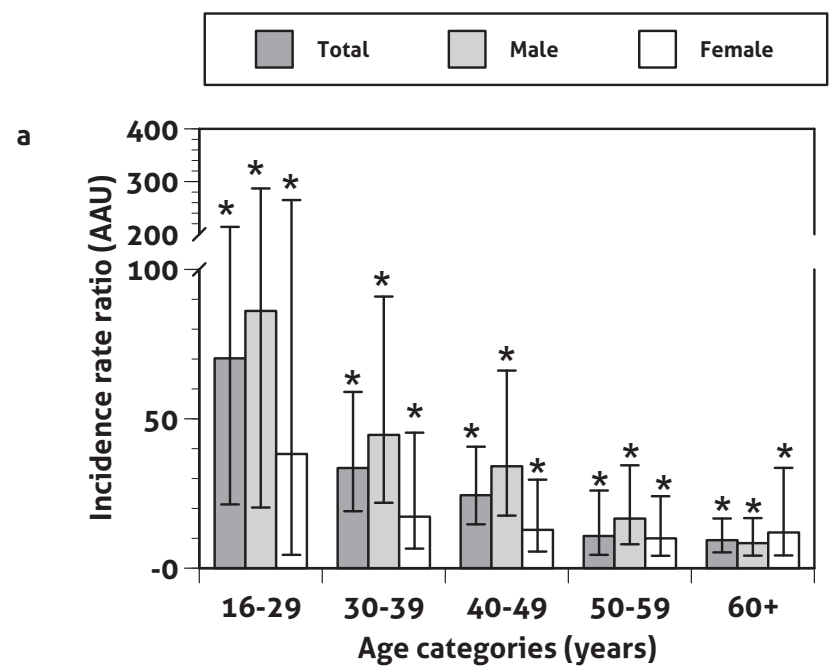

b

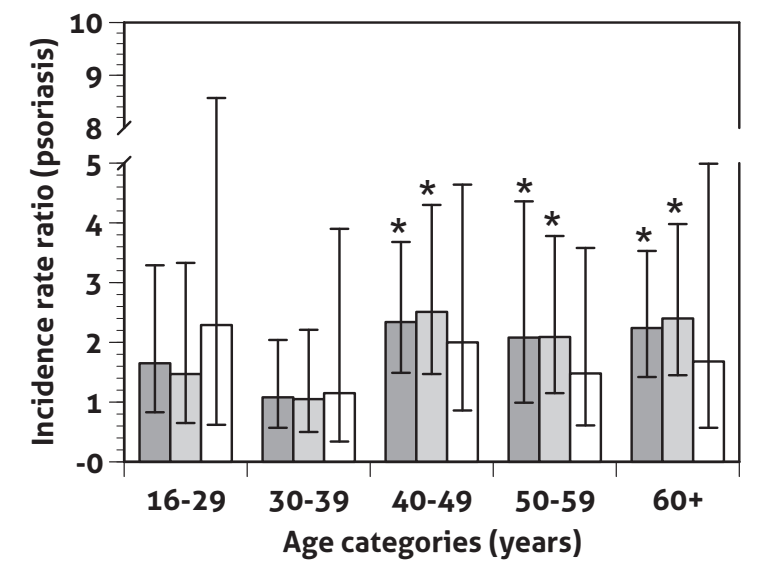

c

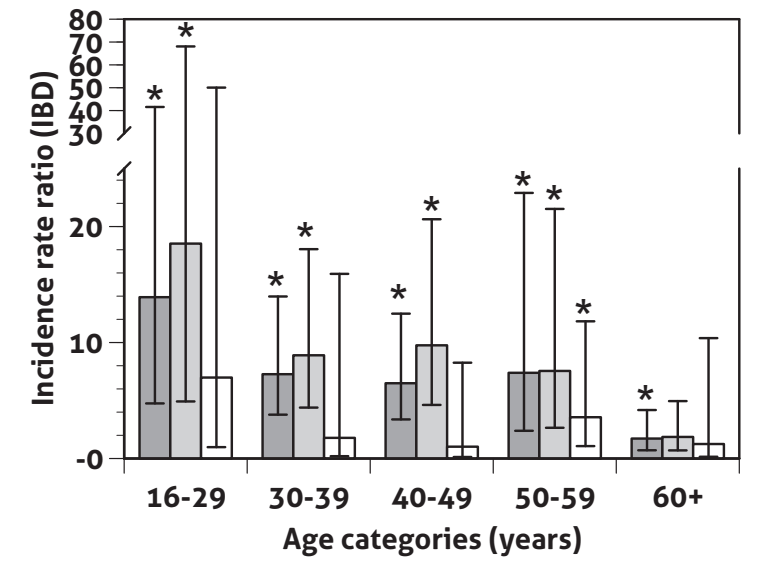

Figure 1 - Incidence rate ratios between patients with AS and controls for AAU (a), psoriasis (b), and IBD (c) according to different age- and sex categories. * Statistically significant. 
a

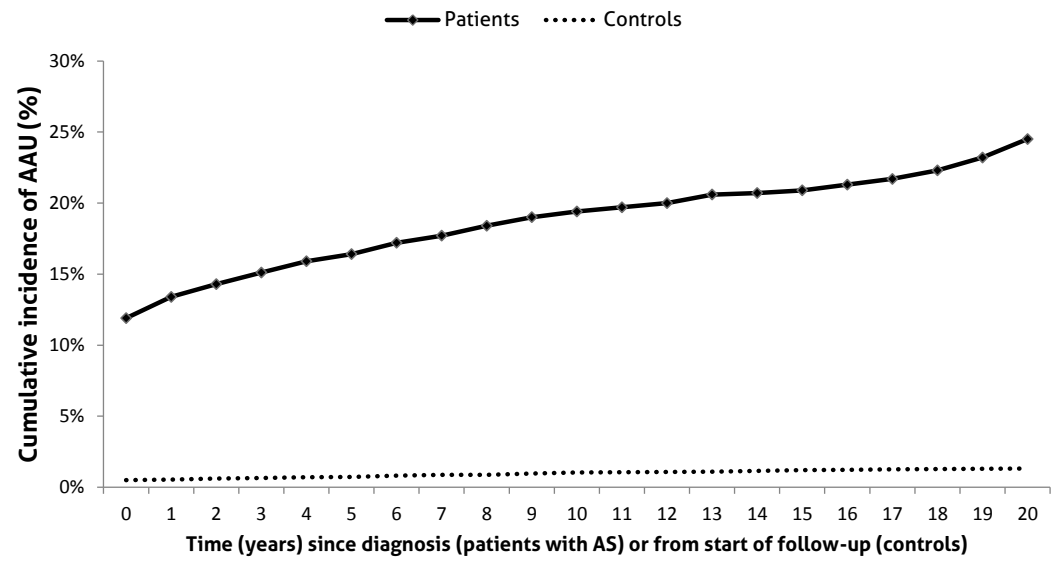

b

- Patients ...... Controls
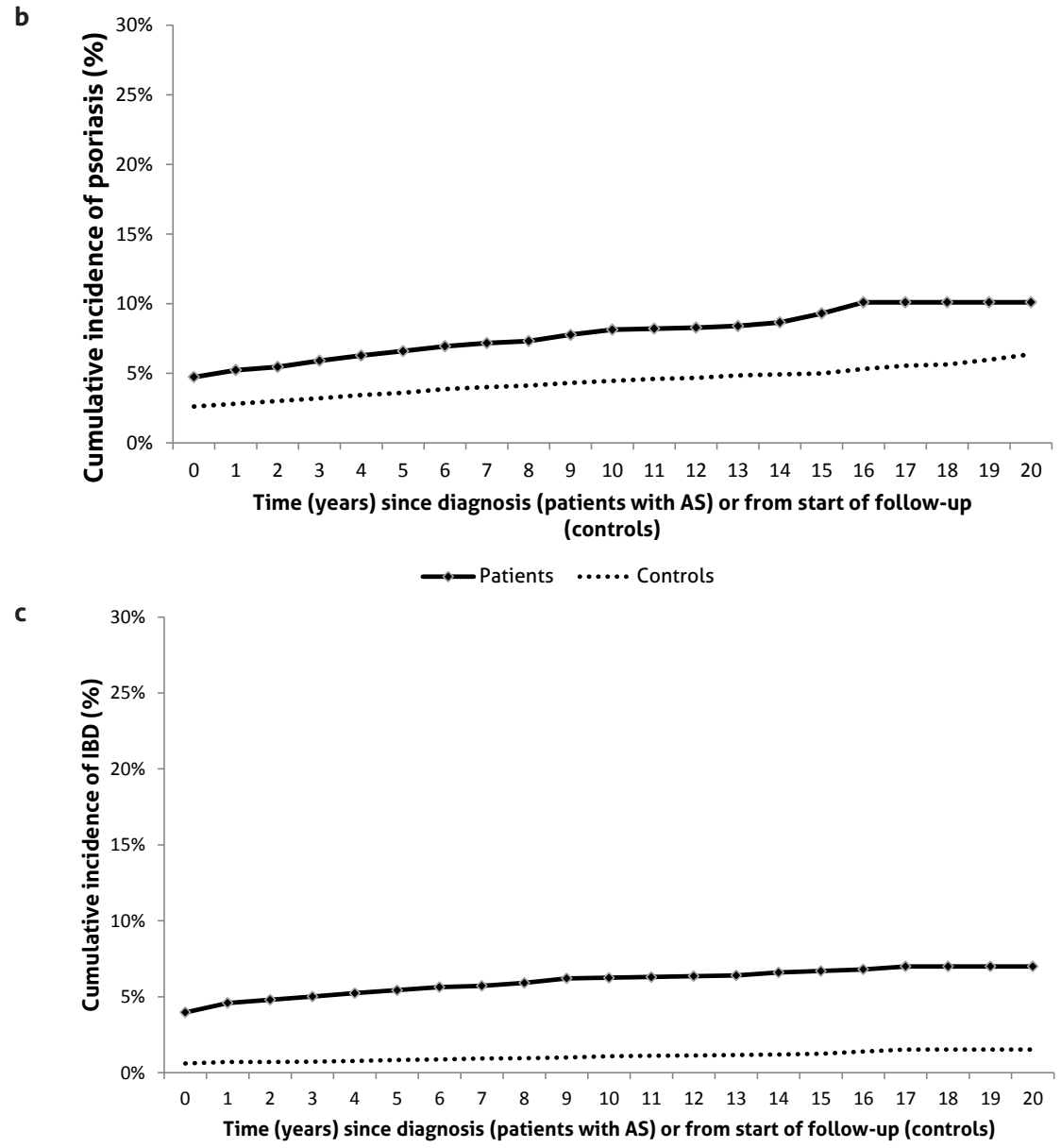

Figure 2 - Cumulative incidence of AAU (a), psoriasis (b) and IBD (c) among patients with AS and matched controls starting from the index date. 
Table 3. Risks of AAU, psoriasis, and IBD in patients with AS, compared with controls

\begin{tabular}{|c|c|c|c|c|c|c|c|c|c|}
\hline & \multicolumn{3}{|c|}{$\operatorname{AAU}(n=3,611)^{*}$} & \multicolumn{3}{|c|}{ Psoriasis $(n=3,907)^{\dagger}$} & \multicolumn{3}{|c|}{$\operatorname{IBD}(n=3,938)^{\ddagger}$} \\
\hline & $\mathrm{n}$ & $\begin{array}{l}\text { Age-sex adj. HR } \\
(95 \% \mathrm{Cl})\end{array}$ & $\begin{array}{l}\text { Full-adj. HR } \\
\text { (95\% Cl) }\end{array}$ & $\mathrm{n}$ & $\begin{array}{l}\text { Age-sex adj. HR } \\
(95 \% \mathrm{Cl})\end{array}$ & $\begin{array}{c}\text { Full-adj. HR }^{b} \\
(95 \% \mathrm{Cl})\end{array}$ & n & $\begin{array}{l}\text { Age-sex adj. HR } \\
(95 \% \mathrm{Cl})\end{array}$ & $\begin{array}{l}\text { Full-adj. HR }{ }^{\mathrm{c}} \\
(95 \% \mathrm{Cl})\end{array}$ \\
\hline No AS & 80 & 1.0 & 1.0 & 341 & 1.0 & 1.0 & 83 & 1.0 & 1.0 \\
\hline AS & 203 & $20.9(16.2-27.1)$ & $15.5(11.6-20.7)$ & 90 & $1.9(1.5-2.4)$ & $1.5(1.1-1.9)$ & 62 & $5.5(3.9-7.6)$ & $3.3(2.3-4.8)$ \\
\hline \multicolumn{10}{|l|}{ Sex } \\
\hline Female & 52 & $13.5(8.6-21.0)$ & $10.7(6.6-17.5)$ & 23 & $1.7(1.1-2.6)$ & $1.5(0.9-2.5)$ & 9 & $2.4(1.1-5.1)$ & $1.2(0.5-2.8)$ \\
\hline Male & 151 & $25.7(18.6-35.4)$ & $18.4(12.9-26.4)$ & 67 & $1.9(1.5-2.5)$ & $1.4(1.1-2.0)$ & 53 & $7.0(4.8-10.1)$ & $4.6(3.0-7.1)$ \\
\hline \multicolumn{10}{|c|}{ Age at index date } \\
\hline $16-29$ & 40 & $34.9(16.9-71.9)$ & $21.1(9.3-47.7)$ & 11 & $1.2(0.6-2.2)$ & $0.7(0.3-1.4)$ & 16 & $10.3(4.8-22.2)$ & $5.7(2.2-14.5)$ \\
\hline $30-39$ & 73 & $43.0(24.3-76.1)$ & $37.3(20.3-68.7)$ & 20 & $1.5(0.9-2.5)$ & $1.1(0.6-1.9)$ & 19 & $6.3(3.4-11.6)$ & $5.0(2.4-10.2)$ \\
\hline $40-49$ & 46 & $16.8(10.2-27.5)$ & $11.3(6.4-19.9)$ & 25 & $2.6(1.7-4.1)$ & $2.2(1.3-3.7)$ & 16 & $6.6(3.4-13.0)$ & $3.3(1.5-7.2)$ \\
\hline $50-59$ & 34 & $17.9(9.9-32.4)$ & $15.1(7.9-28.8)$ & 21 & $2.3(1.4-3.7)$ & $2.2(1.3-3.7)$ & 5 & $2.1(0.8-5.6)$ & $1.2(0.4-3.5)$ \\
\hline$\geq 60$ & 10 & $4.6(2.1-10.0)$ & $3.6(1.6-8.1)$ & 13 & $1.8(1.0-3.2)$ & $1.5(0.8-2.8)$ & 6 & $3.1(1.2-8.1)$ & $2.8(1.0-7.5)$ \\
\hline \multicolumn{10}{|c|}{ Duration since index date } \\
\hline$<1$ years & 53 & $33.5(23.5-47.9)$ & $23.5(15.9-36.6)$ & 18 & $2.6(1.6-4.1)$ & $1.9(1.2-3.1)$ & 22 & $14.0(8.6-22.5)$ & $7.4(4.4-12.6)$ \\
\hline $1-5$ years & 88 & $21.3(15.7-28.9)$ & $16.1(11.6-22.5)$ & 38 & $1.9(1.4-2.7)$ & $1.5(1.1-2.1)$ & 23 & $5.0(3.1-7.9)$ & $3.0(1.8-5.0)$ \\
\hline $5-10$ years & 46 & $18.0(12.5-25.9)$ & $14.1(9.7-20.7)$ & 24 & $1.8(1.2-2.7)$ & $1.4(0.9-2.2)$ & 13 & $3.9(2.2-7.7)$ & $2.7(1.5-4.9)$ \\
\hline$>10$ years & 16 & $11.4(6.6-19.6)$ & $9.2(5.7-16.0)$ & 10 & $1.3(0.7-2.4)$ & $1.1(0.6-2.1)$ & 4 & $2.1(0.8-5.9)$ & $1.5(0.5-4.1)$ \\
\hline
\end{tabular}

$\mathrm{AAU}=$ acute anterior uveitis; $\mathrm{IBD}=$ inflammatory bowel disease; adj=adjusted; $\mathrm{HR}=$ hazard ratio; $\mathrm{Cl}=$ confidence interval; $\mathrm{AS}=$ ankylosing spondylitis

*Only patients without diagnosis of AAU before or at index date

† Only patients without diagnosis of psoriasis before or at index date

‡ Only patients without diagnosis of IBD before or at index date

${ }^{a}$ Adjusted for: age, sex, the use of NSAIDs in the previous six months, number of GP visits in the previous six months

${ }^{b}$ Adjusted for: age, sex, smoking status at index date, alcohol use at index date, the use of antidepressants, antimycotics, coronary vasodilators and antihypertensives in previous six months, history of hypertension, atopic or contact dermatitis and skin infection in previous six months, and the number of GP visits in the previous six months

' Adjusted for: age, sex, smoking status at index date, alcohol use at index date, the use of NSAIDs, antidepressants and anxiolytics/hypnotics in the previous six months, number of GP visits in the previous six months

increased 10 years after index date, the risk had dropped to baseline levels after 5 years for psoriasis and after 10 years for IBD.

\section{Sensitivity analyses}

The results of the sensitivity analysis with a lead-in time of 1 year were comparable to the main analysis for AAU, psoriasis, and IBD. The adj. HRs for AAU, psoriasis, and IBD were 13.7 (95\% Cl, 10.1-18.7), 1.3 (95\% Cl, 1.0-1.8) and 3.1 (95\% Cl, 2.1-4.6), respectively. The risk of AAU was found to be higher in younger (diagnosis of AS at <50 years of age) patients (adj. HR 20.8, 95\% Cl 14.5-29.9) versus older (diagnosis at $\geq 50$ years of age) 
The prevalence of EAMs: a cohort study $\mid 71$

patients (adj. HR 8.5, 95\% Cl 5.2-13.9). This was also found for IBD (adj. HR 4.3, 95\% CI 2.7-6.8 [diagnosis at $<50$ years of age] versus $1.8,95 \% \mathrm{Cl} 0.9-3.7$ [diagnosis at $\geq 50$ years of age]). The risk for psoriasis was not different with age of diagnosis (adj. HR 1.3, 95\% $\mathrm{Cl}$ 0.9-1.8 [diagnosis at $<50$ years of age] versus $1.8,95 \% \mathrm{Cl} 1.2-2.7$ [diagnosis at $\geq 50$ years of age]).

\section{DISCUSSION}

This study showed a 16-fold increased risk for AAU, a 1.5-fold increased risk for psoriasis and a 3.3-fold increased risk for IBD in patients with AS as compared to controls without AS. The risk for AAU remained increased during the course of the disease, whereas the excessive risks for psoriasis and IBD were mainly present in the first years after the index date. EAMs were often already present before the diagnosis of AS: $12 \%$ of patients had a diagnosis of $A A U$, whereas $5 \%$ had a diagnosis of psoriasis, and $4 \%$ a diagnosis of IBD at the index date. Twenty years after the index date, these percentages were roughly doubled to $25 \%, 10 \%$, and $7.5 \%$, respectively.

Our results are slightly different from a retrospective cohort study from Sweden.[17] In that study, Bremander et al. reported age- and sex adjusted standardized morbidity rates (SMRs) for AAU (34.4), psoriasis (2.9), and IBD (9.3), which were higher than the HRs found in the present study. However, the Swedish study did not correct for potential confounders and included both prevalent and incident cases, both for diagnosis of AS and of EAMs. This may have hampered the interpretation and comparison with our results. Of note, the incidences of the different EAMs in the controls found in our study are in line with reported incidences of these conditions in general populations.[20-23]

One of the objectives of our study was to gain more insight in the time of onset of EAMs in relation to the diagnosis of AS. Until now, it was assumed that the prevalence of AAU was positively associated with disease duration, although evidence was only based on cross-sectional data.[15, 16] The present study confirms this association and shows that the cumulative incidence of a first episode of AAU continued to increase more than fifteen years after the index date. The association with a longer duration of the disease and development of either psoriasis or IBD is less clear. In a meta-regression analysis performed by our group, we were unable to show an association between disease duration and the prevalence of psoriasis and IBD, although studies with short disease durations were underrepresented in this analysis.[15] In the present study, we confirmed that the majority of the patients were either diagnosed with psoriasis or IBD before the index date or developed the condition early in the disease course.

The relatively high prevalence of EAMs at the index date found in the present study emphasizes their potential role in the diagnostic process of patients with chronic (in- 
flammatory) back pain. In particular, AAU should raise the suspicion of SpA, since this condition is relatively rare in the general population and rather frequent in patients with SpA. Moreover, it has been shown that AAU has a high positive likelihood ratio (LR 13.9) for the diagnosis of axial SpA in patients with chronic low back pain.[9] The presence of psoriasis and IBD may also contribute to the diagnosis of SpA, although positive LRs were much lower (LR 3.8 and 4.3 resp.).[9] On this line, it is interesting to learn that the findings in the present study confirm those of early SpA cohorts, which also showed high prevalences of EAMs early in the disease. In a German inception cohort including 462 patients with axial SpA, the prevalences of AAU, psoriasis, and IBD were $20.9 \%, 10.2 \%$, and $2.6 \%$, respectively in the subgroup of AS (mean symptom duration 5.2 years).[24] Another inflammatory back pain cohort from France found in the subgroup of 181 newly diagnosed patients with AS (mean symptom duration 1.6 years) prevalences of $11.1 \%$ for AAU, $14.4 \%$ for psoriasis, and $7.2 \%$ for IBD.[25]

The present study has some limitations. First, we cannot exclude misclassification of the diagnosis in a proportion of the patients with AS, which was also shown in a study from Wales among GPs. In that study, $12 \%$ of patients with a diagnosis of AS within the GP dataset had a different diagnosis in the rheumatology dataset and $24 \%$ of patients with an AS code in the rheumatology dataset, were not recorded as having AS using GP records.[26] In our study, the result is probably a non-differential misclassification which may have underestimated the reported risk of EAMs. Also, misclassification of the EAMs is possible. Different studies, however, confirmed the validity of the diagnoses in the CPRD database. For example, it has been shown that the diagnosis of IBD was highly probable or probable in $92 \%$ (95\% Cl 86-96\%) of the cases. [27] Second, a proportion of our patients with AS that we considered as "incident", may have suffered from AS for a longer period of time, either because of a delay in diagnosis or because the first diagnostic code for AS in CPRD did not correlate with the actual diagnostic date of AS. This can be reflected by the relatively high mean age at diagnosis which was 43.7 years. AS is typically diagnosed at an age between 30 and 35 years.[19, 24, 25] Therefore, sensitivity analyses were performed, which showed higher HRs for AAU and IBD in the patient group with an index date before the age of 50. This is in line with our expectations and could be explained by either misclassification of exposure or by the higher risk for a first episode of AAU and IBD at a younger age. Misclassifying prevalent patients as 'incident' may therefore underestimate the risk of EAMs after the diagnosis of AS. Third, we cannot fully exclude diagnostic bias. The relation between AS and the three common EAMs is widely recognized. Therefore, EAMs may be more easily diagnosed in patients with AS as compared with patients without AS, which may have overestimated the risk. Fourth, we did not have information on specific patient and disease characteristics, such as disease activity and HLA-B27 status, which may possibly have influenced the risk of EAMs and would have facilitated identification of patients at risk for an EAM. Moreover, 
prescriptions of biologicals were not included in the CPRD, which may possibly also have influenced the risk of EAMs.[28, 29]

Strengths of this study are that it has a large sample size, and that it is the first study that estimates the relative risks of AAU, psoriasis, and IBD in patients with AS compared with population-based controls, while controlling for possible confounding factors including smoking status and BMI, for which detailed information was available. Further, this study is the first showing longitudinal data on the association of EAMs in relation to the disease course in patients with AS.

In conclusion, this study shows that among patients suffering from AS a significantly increased risk of AAU, psoriasis, and IBD is observed compared with controls, although hazard patterns are different for each of the EAMs. The occurrence of EAMs before the diagnosis of AS confirms their contributory role in the diagnostic process. Given the high risk of all EAMs, awareness of clinicians on EAMs is important in view of treatment choices and impact on quality of life in patients with AS.

\section{SUPPLEMENTARY DATA}

Supplementary data are published on the website of the Annals of the Rheumatic Diseases. 


\section{REFERENCES}

1. Geirsson AJ, Eyjolfsdottir H, Bjornsdottir G, et al. Prevalence and clinical characteristics of ankylosing spondylitis in Iceland - a nationwide study. Clin Exp Rheumatol 2010;28:333-40.

2. Hanova P, Pavelka K, Holcatova I, et al. Incidence and prevalence of psoriatic arthritis, ankylosing spondylitis, and reactive arthritis in the first descriptive population-based study in the Czech Republic. Scand ] Rheumatol 2010;39:310-7.

3. Kaipiainen-Seppanen O, Aho K, Heliovaara M. Incidence and prevalence of ankylosing spondylitis in Finland. J Rheumatol 1997;24:496-9.

4. Braun J, Bollow M, Remlinger G, et al. Prevalence of spondylarthropathies in HLA-B27 positive and negative blood donors. Arthritis Rheum 1998;41:58-67.

5. Braun J, Sieper J. Ankylosing spondylitis. Lancet 2007;369:1379-90.

6. Moll JM, Haslock I, Macrae IF, et al. Associations between ankylosing spondylitis, psoriatic arthritis, Reiter's disease, the intestinal arthropathies, and Behcet's syndrome. Medicine (Baltimore). 1974/09/01 ed1974. p. 343-64.

7. El Maghraoui A. Extra-articular manifestations of ankylosing spondylitis: prevalence, characteristics and therapeutic implications. Eur ] Intern Med 2011;22:554-60.

8. Rudwaleit M, van der Heijde D, Khan MA, et al. How to diagnose axial spondyloarthritis early. Ann Rheum Dis 2004;63:535-43.

9. Molto A, Paternotte S, Comet D, et al. Performances of the Assessment of SpondyloArthritis International Society Axial Spondyloarthritis Criteria for Diagnostic and Classification Purposes in Patients Visiting a Rheumatologist Because of Chronic Back Pain: Results From a Multicenter, Cross-Sectional Study. Arthritis Care Res (Hoboken) 2013:65:1472-81.

10. Amor B, Dougados M, Mijiyawa M. [Criteria of the classification of spondylarthropathies]. Rev Rhum Mal Osteoartic 1990;57:85-9.

11. Dougados M, van der Linden S, Juhlin R, et al. The European Spondylarthropathy Study Group preliminary criteria for the classification of spondylarthropathy. Arthritis Rheum 1991;34:1218-27.

12. Rudwaleit M, van der Heijde D, Landewe R, et al. The development of Assessment of SpondyloArthritis international Society classification criteria for axial spondyloarthritis (part II): validation and final selection. Ann Rheum Dis 2009;68:777-83.

13. Rudwaleit M, van der Heijde D, Landewe R, et al. The Assessment of SpondyloArthritis International Society classification criteria for peripheral spondyloarthritis and for spondyloarthritis in general. Ann Rheum Dis 2011;70:25-31.

14. Braun J, van den Berg R, Baraliakos X, et al. 2010 update of the ASAS/EULAR recommendations for the management of ankylosing spondylitis. Ann Rheum Dis 2011;70:896-904.

15. Stolwijk C, Tubergen van A, Castillo-Ortiz J, et al. Prevalence of extra-articular manifestations in patients with ankylosing spondylitis: a systematic review and meta-analysis. Ann Rheum Dis 2013.

16. Zeboulon N, Dougados M, Gossec L. Prevalence and characteristics of uveitis in the spondyloarthropathies: a systematic literature review. Ann Rheum Dis 2008;67:955-9.

17. Bremander A, Petersson IF, Bergman S, et al. Population-based estimates of common comorbidities and cardiovascular disease in ankylosing spondylitis. Arthritis Care Res (Hoboken) 2011;63:550-6.

18. Khan NF, Harrison SE, Rose PW. Validity of diagnostic coding within the General Practice Research Database: a systematic review. Br ] Gen Pract 2010;60:e128-36.

19. Feldtkeller $E$, Khan MA, van der Heijde $D$, et al. Age at disease onset and diagnosis delay in HLA-B27 negative vs. positive patients with ankylosing spondylitis. Rheumatol Int 2003;23:61-6.

20. Gritz DC, Wong IG. Incidence and prevalence of uveitis in Northern California; the Northern California Epidemiology of Uveitis Study. Ophthalmology 2004;111:491-500; discussion

21. Paivonsalo-Hietanen T, Tuominen J, Vaahtoranta-Lehtonen $\mathrm{H}$, et al. Incidence and prevalence of different uveitis entities in Finland. Acta Ophthalmol Scand 1997;75:76-81. 
22. Loftus EV, Jr. Clinical epidemiology of inflammatory bowel disease: Incidence, prevalence, and environmental influences. Gastroenterology 2004;126:1504-17.

23. Parisi R, Symmons DP, Griffiths CE, et al. Global epidemiology of psoriasis: a systematic review of incidence and prevalence. J Invest Dermatol 2013:133:377-85.

24. Rudwaleit $M$, Haibel $H$, Baraliakos $X$, et al. The early disease stage in axial spondylarthritis: results from the German Spondyloarthritis Inception Cohort. Arthritis Rheum 2009;60:717-27.

25. Dougados M, d'Agostino MA, Benessiano J, et al. The DESIR cohort: a 10-year follow-up of early inflammatory back pain in France: study design and baseline characteristics of the 708 recruited patients. Joint Bone Spine 2011;78:598-603.

26. Brophy S, Cooksey R, Atkinson M, et al. No increased rate of acute myocardial infarction or stroke among patients with ankylosing spondylitis-a retrospective cohort study using routine data. Semin Arthritis Rheum 2012;42:140-5.

27. Lewis JD, Brensinger C, Bilker WB, et al. Validity and completeness of the General Practice Research Database for studies of inflammatory bowel disease. Pharmacoepidemiol Drug Saf 2002;11:211-8.

28. Braun J, Baraliakos X, Listing J, et al. Decreased incidence of anterior uveitis in patients with ankylosing spondylitis treated with the anti-tumor necrosis factor agents infliximab and etanercept. Arthritis Rheum 2005;52:2447-51.

29. Braun J, Baraliakos X, Listing J, et al. Differences in the incidence of flares or new onset of inflammatory bowel diseases in patients with ankylosing spondylitis exposed to therapy with anti-tumor necrosis factor alpha agents. Arthritis Rheum 2007;57:639-47. 



\section{CHAPTER 5}

\section{Prevalence of self-reported spondyloarthritis features in a cohort of patients with inflammatory bowel disease}

Carmen Stolwijk, Marieke Pierik, Robert Landewé, Ad Masclee, Astrid van Tubergen 


\section{ABSTRACT}

\section{Background}

Musculoskeletal symptoms belonging to the spectrum of "seronegative spondyloarthritis" (SpA) are the most common extraintestinal manifestations in patients with inflammatory bowel disease (IBD) and may cause important disease burden. Patients suspected with SpA should be referred to a rheumatologist for further evaluation.

\section{Objective}

To investigate the self-reported prevalence of musculoskeletal SpA features in a cohort of patients with IBD and to compare this with the actual referrals to a rheumatologist.

\section{Methods}

Patients with IBD consecutively visiting the outpatient clinic were interviewed by a trained research nurse about possible SpA features using a standardized questionnaire on the presence or history of inflammatory back pain, peripheral arthritis, enthesitis, dactylitis, psoriasis, uveitis and response to NSAIDs. All patient files were checked for prior visits to a rheumatologist and any rheumatic diagnosis.

\section{Results}

At least one musculoskeletal SpA feature was reported by 129 out of 350 (36.9\%) patients. No significant differences between patients with Crohn's disease and ulcerative colitis were found. Review of medical records showed that 66 (51.2\%) patients ever visited a rheumatologist. Axial SpA was diagnosed in 18 (27.3\%) patients, peripheral SpA in $20(30.3 \%)$ patients, and another rheumatic disorder in $14(21.2 \%)$ patients.

\section{Conclusion}

Musculoskeletal SpA features are frequently present in patients with IBD. However, a substantial group of patients is not evaluated by a rheumatologist. Gastroenterologists play a key role in early referral of this often debilitating disease. 


\section{INTRODUCTION}

In patients with inflammatory bowel disease (IBD), musculoskeletal symptoms are the most common extraintestinal manifestations [1]. Arthritis and spondylitis associated with IBD belong to the spectrum of "seronegative spondyloarthritis" (SpA) [2]. SpA is a group of disorders that share several clinical features, show familial clustering, and are linked to the human leukocyte antigen-B27 (HLA-B27). The major subtypes of the SpA group are ankylosing spondylitis, psoriatic arthritis, reactive arthritis, arthritis/spondylitis associated with IBD, and undifferentiated SpA. According to their clinical presentation, patients with SpA can be divided into patients with predominantly axial symptoms and patients with predominantly peripheral symptoms [3]. Axial involvement consists of inflammatory back pain reflecting inflammation of the sacroiliac joints and/or spine. Peripheral involvement consists of peripheral arthritis, dactylitis ('sausage-like' finger or toe) and enthesitis (frequently at the insertion of the Achilles tendon or the plantar fascia).

In daily practice, SpA symptoms are not always recognized in patients with IBD. To most patients the relationship between joint and gut symptoms is unknown, and gastroenterologists not always specifically ask for joint involvement. Subsequently, patients with symptoms of SpA may be underdiagnosed and effective treatment delayed, which may lead to a chronic debilitating disease course and decreased quality of life [4]. To date, several studies have shown that dramatic improvements in disease activity and functioning can be achieved with anti-Tumour Necrosis Factor-alpha (anti-TNF-a) treatment in patients with several forms of SpA, including the early stages of axial SpA [5-10]. It has also been demonstrated that remission of symptoms with anti-TNF-a treatment can be achieved in a higher percentage of patients when treated early in the disease course and at a young age $[11,12]$. Recognition and intervention of the disease at an early stage is therefore warranted.

Diagnosing SpA is not always easy and diagnostic criteria are currently lacking. Several criteria sets are available for classification of (subgroups of) SpA, but these have been developed mainly for study purposes. Ankylosing spondylitis, as the prototype of SpA, is classified by the modified New-York criteria [13]. In this classification set, radiographic sacroiliitis is essential, together with the presence of at least one clinical criterion. However, it can take many years before sacroiliitis is visible on pelvic radiographs, resulting in a mean diagnostic delay of 6-8 years [14]. In the early 1990s, two other criteria sets were developed to classify patients with SpA: the European Spondyloarthropathy group (ESSG) criteria [15] and the Amor criteria [16]. The ESSG and Amor criteria perform well in groups of patients with a definite diagnosis of SpA [17-19], but also lack diagnostic value in patients with early, mild or 'possible' SpA [19, 20].

Recently, an international group of experts in the field of SpA -the Assessment of SpondyloArthritis international Society (ASAS)- generated two new sets of criteria for 
In patients with $\geq 3$ months back pain

(with / without peripheral manifestations)

and age at onset $<45$ years:

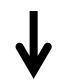

\begin{tabular}{|c|c|}
\hline $\begin{array}{c}\text { Sacroiliitis on imaging } \\
\text { plus } \geq 1 \text { SpA feature }\end{array}$ & OR $\begin{array}{c}\text { HLA-B27 plus } \geq 2 \text { other } \\
\text { SpA features }\end{array}$ \\
\hline
\end{tabular}

SpA features

- inflammatory back pain (IBP)

- Arthritis

- Enthesitis (heel)

- Uveitis

- Dactylitis

- Psoriasis

- Crohn's disease / Ulcerative colitis

- Good response to NSAIDs

- Family history for SpA

- HLA-B27

- Elevated CRP

*Peripheral arthritis: usually predominantly lower limbs and/or asymmetric arthritis Combined sensitivity $79.5 \%$, combined specificity $83.3 \%, n=975$

\section{In patients with peripheral manifestations ONLY:}

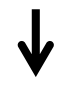

Arthritis or enthesitis or dactylitis plus

$\geq 1$ SpA feature

- Uveitis

- Psoriasis

- Crohn's disease / Ulcerative colitis

- Preceding infection

- HLA-B27

- Sacroiliitis on imaging

OR

$\geq 2$ other SpA features

- Arthritis

- Enthesitis

- Dactylitis

- IBP ever

- Family history for SpA

Figure 1 - Assessment of SpondyloArthritis international Society (ASAS) criteria for axial and peripheral spondyloarthritis.

the classification of SpA: one for patients with predominantly axial symptoms and one for patients with predominantly peripheral symptoms (figure 1) [21, 22]. Both criteria sets have been developed in order to capture also the early and mild cases of SpA and include several SpA features. These features can easily be asked for in daily practice, also by gastroenterologists, in order to recognize patients possibly suffering from SpA.

The aim of this study was first to investigate in a large cohort of patients with IBD the self-reported prevalence of musculoskeletal SpA features as included in the new ASAS criteria sets in order to get a better understanding of the size of this concomitant disease in daily practice, and second to compare the self-reported prevalence with the actual referrals to a rheumatologist and the final diagnosis in these referred patients.

\section{METHODS}

Patients included in the present study participated in an ongoing cohort of patients with IBD (IBD South-Limburg cohort). The diagnosis of IBD, made by gastroenterologists, was based on clinical, endoscopic, and histological evaluation. For the present study, all patients from the IBD South-Limburg cohort who consecutively visited the outpatient clinic of the Maastricht University Medical Center, The Netherlands, between October 2009 and June 2011 were interviewed by a trained research nurse about possible SpA features. A 
standardized questionnaire was used containing the following features from the ASAS criteria: presence or history of inflammatory back pain; duration of inflammatory back pain; (history of) peripheral arthritis; (history of) enthesitis (history of Achilles tendinitis, plantar fasciitis or inflammation of the anterior chest wall); (history of) dactylitis (history of a 'sausage-like digit'); psoriasis; (history of) uveitis; response of arthritis or inflammatory back pain to NSAIDs, and a family history of SpA. Inflammatory back pain was defined as low back pain existing for more than three months, which started before the age of 45, is worst in the early morning and improves with exercise. From the database of the IBD South-Limburg cohort, information was extracted about age, gender, diagnosis (Crohn's disease (CD), ulcerative colitis (UC) or IBD unclassified (IBDU)), duration of the inflammatory bowel disease, current use of medication for IBD and IBD disease activity. IBD disease activity was calculated using the Harvey-Bradshaw index ( $\mathrm{HBI}$ ) for patients with CD (range 0 to infinite; score $<5$ is defined as CD in remission, a score $>15$ as severe disease) and the simple clinical colitis activity index (SCCAI) for patients with either UC or IBDU (range 0 to 20; score $>4$ is suggestive for active colitis) [23, 24]. Because joint symptoms are part of these disease activity scores (counting for one point if present), the total scores for both the $\mathrm{HBI}$ and the SCCAI were also recalculated excluding this item. All patient files were checked for prior visits to a rheumatologist and any rheumatic diagnosis (axial or peripheral SpA or any other rheumatic disease). The study was approved by the ethical committee from the Maastricht University Medical Center.

\section{Statistics}

Descriptive statistics were used to calculate the mean with standard deviation (SD) for continuous data. Independent t-tests and chi square tests were used to compare differences between the groups for continuous and dichotomous data, respectively. Univariable followed by multivariable logistic regression analyses were applied to identify associations between any reported SpA feature and demographic and clinical variables. Similar analyses were performed to identify associations between a definite diagnosis of SpA and these variables. In multivariable analyses, models were stratified for diagnosis of IBD (CD or UC), due to the different disease activity scores for CD and UC. In order to investigate the relation between either self-reported peripheral or axial SpA features or the definite diagnosis of peripheral or axial SpA with duration of IBD, the cohort was subdivided in quartiles according to duration of IBD and subsequently the frequency of self-reported SpA features and diagnosis of SpA was calculated per quartile. Logistic regression analyses were performed to test the relation between the disease duration of IBD (in quartiles) and the frequency of reported (peripheral or axial) SpA features or diagnosis of (peripheral or axial) SpA. In patients who reported at least one musculoskeletal SpA feature, associations between the individual SpA symptoms and referral to a rheumatologist were identified in univariable followed by multivariable 
logistic regression analysis while controlling for demographic and disease characteristics. All logistic regression analyses were carried out in a stepwise backward likelihood ratio method. Possible interactions between the variables were tested in separate analyses. All analyses were performed using SPSS 16.0 software. The significance level was set at 0.05.

\section{RESULTS}

\section{Patient characteristics}

In total 365 consecutive patients with IBD who visited the outpatient clinic between October 2009 and June 2011 were asked to participate in the IBD South-Limburg cohort of whom 350 (95.9\%) patients agreed. All 350 patients were interviewed about SpA features. Patient characteristics and self-reported SpA features are shown in Table 1. Of the 350 patients, 206 suffered from CD, 136 from UC and 8 from IBDU. Patients with CD were younger, more frequently using a biological, and more frequently female compared with patients with UC.

\section{Self-reported SpA features}

At least one musculoskeletal SpA feature was reported by 129 out of 350 (36.9\%) patients. Seventy-nine (22.6\%) patients reported axial symptoms and 83 (23.7\%) patients reported at least one peripheral SpA feature. There were no statistically significant differences between patients with CD and UC with regard to self-reported SpA features. Figure $2 \mathrm{a}$ shows the relation between self-reported axial or peripheral SpA features and duration of IBD. A trend towards more axial and peripheral SpA features with longer disease duration was found, although not statistically significant $(p=0.28$ and $p=0.18$, respectively).

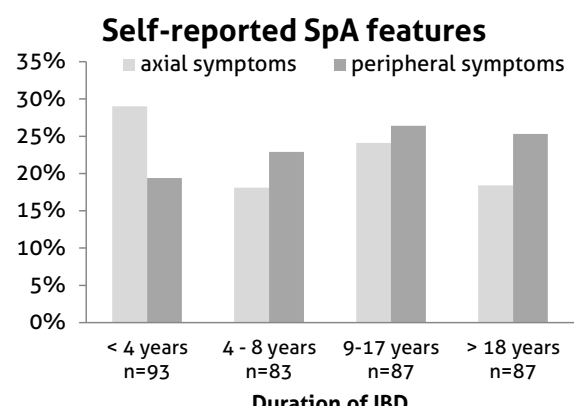

a

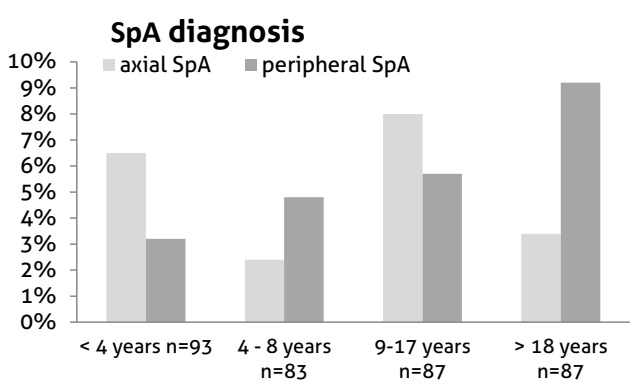

b

Duration of IBD

Figure 2 - Presence of self-reported SpA features (a) and diagnosis of either axial or peripheral SpA (b) per quartile of duration of IBD. IBD: inflammatory bowel disease, SpA: spondyloarthritis 
Table 1. Patients characteristics and prevalence of SpA features in patients with IBD

\begin{tabular}{|c|c|c|c|c|c|}
\hline & $\begin{array}{l}\text { Total IBD } \\
(n=350)\end{array}$ & $\begin{array}{c}C D \\
(n=206)\end{array}$ & $\begin{array}{c}\text { UC } \\
(n=136)\end{array}$ & $\begin{array}{l}\text { IBDU } \\
(n=8)\end{array}$ & $\begin{array}{c}\text { P-value } \\
\text { (CD vs. UC) }\end{array}$ \\
\hline Mean age (years $\pm S D$ ) & $46.6 \pm 15.5$ & $43.8 \pm 14.9$ & $50.5 \pm 15.2$ & $52.4 \pm 21.6$ & $0.000^{A}$ \\
\hline Female gender & $197(56.3)$ & $128(62.1)$ & $65(47.8)$ & $4(50.0)$ & 0.009 \\
\hline Mean duration of IBD (years $\pm S D$ ) & $11.4 \pm 10.1$ & $11.6 \pm 10.7$ & $11.4 \pm 9.5$ & $7.5 \pm 4.2$ & 0.847 \\
\hline Mean IBD activity (score $\pm S D)^{B}$ & - & $3.3 \pm 3.5$ & $2.7 \pm 2.7$ & $0.9 \pm 0.9$ & - \\
\hline Mean IBD activity excluding joint symptoms (score $\pm S D)^{B}$ & - & $3.1 \pm 3.5$ & $1.7 \pm 2.7$ & $0.2 \pm 0.4$ & - \\
\hline Currently using medication for IBD & $291(83.1)$ & $172(83.5)$ & $111(81.6)$ & $8(100)$ & 0.653 \\
\hline - Thiopurines & $100(28.6)$ & $75(36.4)$ & $23(16.9)$ & $2(25)$ & $0.000^{A}$ \\
\hline - Mesalazine & $133(38)$ & $46(22.3)$ & $82(60.3)$ & $5(62.5)$ & $0.000^{A}$ \\
\hline - Methotrexate & $11(3.1)$ & $10(4.9)$ & $1(0.7)$ & 0 & $0.035^{A}$ \\
\hline - Corticosteroids & $33(9.4)$ & $23(11.2)$ & $10(7.4)$ & 0 & 0.243 \\
\hline - Biologials & $84(24)$ & $68(33)$ & $15(11)$ & $1(12.5)$ & $0.000^{A}$ \\
\hline Psoriasis & $27(7.7)$ & $16(7.8)$ & $11(8.1)$ & 0 & 0.914 \\
\hline Uveitis & $5(1.4)$ & $5(2.4)$ & 0 & 0 & 0.059 \\
\hline Family history of SpA & $159(45.4)$ & $96(46.6)$ & $58(42.6)$ & $4(50.0)$ & 0.483 \\
\hline Family history of IBD & $105(30.0)$ & $64(31.1)$ & $37(27.2)$ & $4(50.0)$ & 0.418 \\
\hline Any musculoskeletal SpA feature & $129(36.9)$ & $82(39.8)$ & $45(33.1)$ & $2(25.0)$ & 0.208 \\
\hline Inflammatory back pain & $79(22.6)$ & $49(23.8)$ & $29(21.3)$ & $1(12.5)$ & 0.435 \\
\hline$-<2$ years & $13(3.7)$ & $9(4.4)$ & $4(2.9)$ & 0 & 0.600 \\
\hline$-2-10$ years & $24(6.9)$ & $13(6.3)$ & $11(8.1)$ & 0 & 0.292 \\
\hline - >10 years & $42(12.0)$ & $27(13.1)$ & $14(10.3)$ & $1(12.5)$ & 0.560 \\
\hline Any peripheral SpA feature & $83(23.7)$ & $54(26.2)$ & $27(19.9)$ & $1(12.5)$ & 0.176 \\
\hline Enthesitis & $47(13.4)$ & $30(14.6)$ & $16(11.8)$ & $1(12.5)$ & 0.458 \\
\hline Dactylitis & $29(8.3)$ & $19(9.2)$ & $10(7.4)$ & 0 & 0.543 \\
\hline Peripheral arthritis & $33(9.4)$ & $25(12.1)$ & $8(5.1)$ & 0 & 0.054 \\
\hline Diagnosis of axial SpA by rheumatologist & $18(5.1)$ & $13(6.3)$ & $5(3.7)$ & 0 & 0.251 \\
\hline Diagnosis of peripheral SpA by rheumatologist & $20(5.7)$ & $15(7.3)$ & $5(3.7)$ & 0 & 0.147 \\
\hline
\end{tabular}

Data are expressed as number (\%) unless stated otherwise. SpA: spondyloarthritis

A Statistically significant; ${ }^{B}$ Harvey-Bradshaw index in patients with CD (data available in 184 patients); Simple clinical colitis activity index in patients with UC and IBDU (data available in 116 patients).

Table 2 shows the results from the regression analyses investigating the association between demographic and clinical variables on the presence of self-reported SpA features. The regression analysis was performed with data from 342 patients: the 8 patients with IBDU were excluded. In the univariable analysis, male gender was associated with less frequently reported SpA features, whereas use of biologicals and a higher mean disease activity score (the latter only in patients with $C D$ ) were associated with more frequently reported SpA features. When joint symptoms were excluded in the disease activity score, the score remained significantly associated with self-reported SpA features. In multivari- 
Table 2. Comparisons between patients with and without reported SpA features and with and without SpA classification

\begin{tabular}{|c|c|c|c|c|c|c|}
\hline & \multicolumn{3}{|c|}{ Musculoskeletal SpA features } & \multicolumn{3}{|c|}{ Axial or peripheral SpA classification } \\
\hline & $\begin{array}{l}\text { Present } \\
(n=129)\end{array}$ & $\begin{array}{c}\text { Absent } \\
(n=221)\end{array}$ & P-value & $\begin{array}{l}\text { Present } \\
(n=38)\end{array}$ & $\begin{array}{c}\text { Absent } \\
(n=312)\end{array}$ & P-value \\
\hline Mean age (years \pm SD) & $47.0 \pm 14.4$ & $46.5 \pm 16.2$ & 0.733 & $46.4 \pm 11.6$ & $46.6 \pm 16.0$ & 0.914 \\
\hline Female gender & $83(64.3)$ & $114(51.6)$ & $0.02^{\mathrm{A}}$ & $23(60.5)$ & $174(55.8)$ & 0.577 \\
\hline Mean duration of IBD (years \pm SD) & $11.5 \pm 10.3$ & $11.4 \pm 10.1$ & 0.947 & $12.9 \pm 10.4$ & $11.2 \pm 10.1$ & 0.339 \\
\hline Diagnosis (CD/UC/IBDU) & $\begin{array}{c}82 / 45 / 2 \\
(63.6 / 34.9 / 1.6)\end{array}$ & $\begin{array}{c}124 / 91 / 6 \\
(56.1 / 41.2 / 2.7)\end{array}$ & 0.353 & $\begin{array}{c}28 / 10 / 0 \\
(73.7 / 26.3 / 0)\end{array}$ & $\begin{array}{c}178 / 126 / 8 \\
(57.1 / 40.4 / 2.6)\end{array}$ & 0.117 \\
\hline Currently using medication for IBD & $104(80.6)$ & $187(84.6)$ & 0.335 & $31(81.6)$ & $260(83.3)$ & 0.785 \\
\hline - Biologicals & $39(30.2)$ & $45(20.4)$ & $0.037^{A}$ & $12(31.6)$ & $72(23.1)$ & 0.247 \\
\hline Mean $\mathrm{HBI}(\text { score } \pm \mathrm{SD})^{\mathrm{B}}$ & $4.1 \pm 3.7$ & $2.7 \pm 3.2$ & $0.04^{\mathrm{A}}$ & $4.0 \pm 2.9$ & $3.1 \pm 3.6$ & 0.269 \\
\hline $\begin{array}{l}\text { Mean HBI excluding joint symptoms } \\
(\text { score } \pm \text { SD) }\end{array}$ & $3.8 \pm 3.7$ & $2.6 \pm 3.2$ & $0.019^{A}$ & $3.5 \pm 2.8$ & $3.1 \pm 3.5$ & 0.555 \\
\hline Mean SSCAI (score \pm SD) ${ }^{C}$ & $1.9 \pm 2.5$ & $1.6 \pm 2.8$ & 0.58 & $2.4 \pm 1.4$ & $1.6 \pm 2.7$ & 0.456 \\
\hline $\begin{array}{l}\text { Mean SSCAI excluding joint } \\
\text { symptoms (score } \pm \text { SD) }\end{array}$ & $1.7 \pm 2.5$ & $1.6 \pm 2.8$ & 0.861 & $1.6 \pm 1.2$ & $1.6 \pm 2.8$ & 0.989 \\
\hline Family history of SpA & $62(48.1)$ & $96(43.4)$ & 0.444 & $21(55.3)$ & $137(43.9)$ & 0.196 \\
\hline Family history IBD & $40(31.0)$ & $65(29.4)$ & 0.723 & $13(34.2)$ & $92(29.5)$ & 0.583 \\
\hline Uveitis & $3(2.3)$ & $2(0.9)$ & 0.282 & $3(7.9)^{A}$ & $2(0.6)$ & $0.001^{A}$ \\
\hline Psoriasis & $14(10.9)$ & $13(5.9)$ & 0.093 & $5(13.2)$ & $22(7.1)$ & 0.183 \\
\hline
\end{tabular}

Data are expressed as number (\%) unless otherwise stated. SpA: spondyloarthritis

A Statistically significant, ${ }^{\text {B }} \mathrm{HBI}$ : Harvey-Bradshaw index (CD) (data available in 184 patients), ${ }^{\text {C SSCAl: }}$ Simple clinical colitis activity index (UC and IBDU) (data available in116 patients)

able analysis, which was stratified for IBD diagnosis, male gender (OR $0.44,95 \% \mathrm{Cl} 0.23$ to 0.84 ) and disease activity score (OR $1.14,95 \% \mathrm{Cl} 1.03$ to 1.24 ) were both significantly associated with the presence of reported SpA features in patients with CD. In patients with UC, none of the variables was associated with self-reported SpA features. Interaction between the variables was not found.

\section{Referrals to rheumatologist and final diagnosis}

Review of the medical-records of all 350 patients showed that 66 (51.2\%) out of 129 patients, who reported at least one musculoskeletal SpA feature, were ever seen by a rheumatologist in our hospital (figure 3). Table 3 shows the regression analysis investigating which individual SpA symptoms were associated with a referral to a rheumatologist in patients who reported at least one musculoskeletal SpA feature, while controlling for demographic and disease characteristics. In the multivariable analysis, inflammatory back pain (OR 8.97, 95\% Cl 2.48 to 32.45), peripheral arthritis (OR 44.56, 95\% Cl 8.57 to 231.56) and enthesitis (OR 4.02,95\% Cl 1.22 to 13.27) were all independently associated with referral to a rheumatologist, whereas dactylitis was not. Interaction between the variables was not found. In the patients who were seen by a rheumatologist, axial 


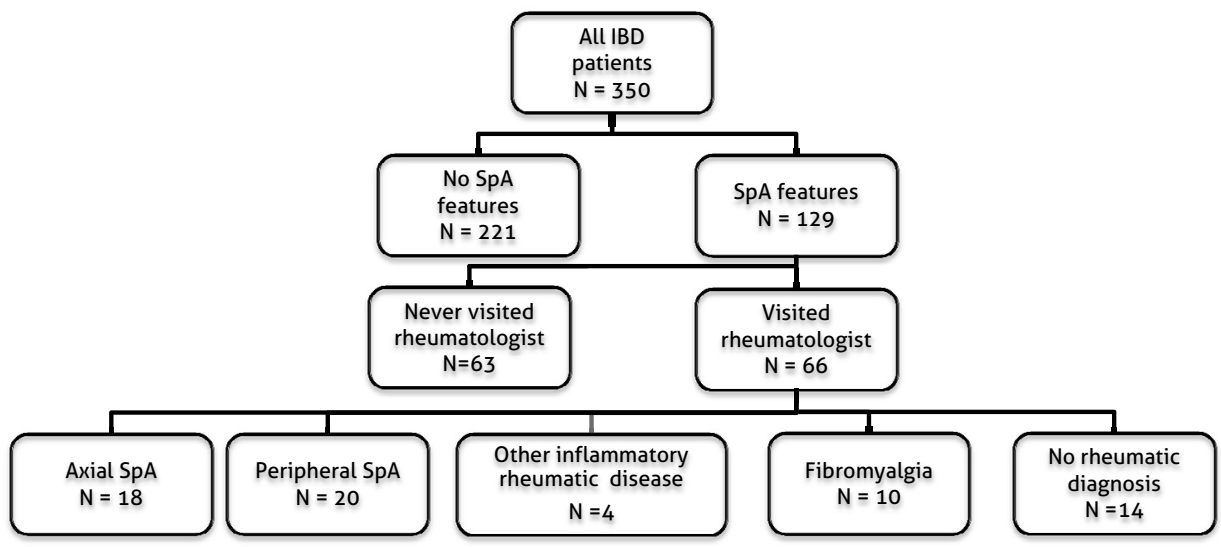

Figure 3 - Flow-chart of all 350 patients with IBD included in the present study.

Table 3. Univariable and multivariable logistic regression analysis investigating the association between individual SpA symptoms and referral to a rheumatologist

\begin{tabular}{|c|c|c|c|c|}
\hline \multirow[b]{2}{*}{ Variables } & \multicolumn{2}{|l|}{ Univariable analysis } & \multicolumn{2}{|l|}{ Multivariable analysis } \\
\hline & Odds ratio ( $95 \% \mathrm{Cl})$ & p Value & Odds ratio $(95 \% \mathrm{Cl})$ & p Value \\
\hline Inflammatory back pain & $1.98(0.96 ; 4.08)$ & 0.07 & $8.97(2.48 ; 32.45)$ & $<0.01$ \\
\hline Peripheral arthritis & $17.88(5.07 ; 63.09)$ & $<0.01$ & $44.56(8.57 ; 231.56)$ & $<0.01$ \\
\hline Enthesitis & $1.22(0.59 ; 2.52)$ & 0.59 & $4.02(1.22 ; 13.27)$ & 0.02 \\
\hline Dactylitis & $1.23(0.54 ; 2.84)$ & 0.63 & - & - \\
\hline Uveitis & $0.00(0.00 ; 0.00)$ & 1.00 & - & - \\
\hline Psoriasis & $1.83(0.58 ; 5.81)$ & 0.30 & - & - \\
\hline
\end{tabular}

IBD: inflammatory bowel disease, CD: Crohn's disease. By default, the multivariable model was adjusted for age, disease duration, gender and diagnosis

SpA was diagnosed in 18 (27.3\%) patients and peripheral SpA in 20 (30.3\%) patients. Fourteen out of $66(21.2 \%)$ patients suffered from another rheumatic disorder (3 rheumatoid arthritis, 1 juvenile inflammatory arthritis and 10 fibromyalgia) and in 14 (21.2\%) patients no rheumatic disorder was diagnosed. Figure $2 \mathrm{~b}$ shows the relation between the duration of IBD and a final diagnosis of axial or peripheral SpA. Visually, a trend towards an increase in peripheral SpA diagnosis with increasing duration of IBD was found. However, this relation was not statistically significant $(p=0.09)$. For axial SpA diagnosis, no association with disease duration was found ( $p=0.73$ ). Table 2 also shows the logistic regression analysis investigating variables associated with a final diagnosis of SpA. In patients with CD, uveitis was independently associated with a diagnosis of SpA (OR 9.06, 95\% 1.44 to 57.10 ). In patients with UC, psoriasis was associated with a diagnosis of SpA (OR $6.32,95 \% \mathrm{Cl} 1.37$ to 29.20 ). 


\section{DISCUSSION}

The present study showed that more than one third of 350 patients from an unselected IBD cohort reported musculoskeletal SpA features as included in the new ASAS criteria. Of these patients, only $51 \%$ were ever seen by a rheumatologist. Axial or peripheral SpA was diagnosed in $58 \%$ of the patients who were seen by a rheumatologist, and in $21 \%$ of the patients, another rheumatic disorder was diagnosed. Strikingly, almost $50 \%$ of the patients who reported musculoskeletal symptomatic patients never visited a rheumatologist.

Referral to a rheumatologist is important, because SpA may result in significant impairment in several aspects of quality of life and restrictions in social roles, including work participation [25]. The disease course of axial SpA begins with inflammation of the sacroiliac joints. Disease progression is characterized by the development of (irreversible) structural damage of the sacroiliac joints and the spine, which is associated with worse physical function and limitation of spinal mobility [26]. Importantly, patients with early axial SpA are not different from those with definite ankylosing spondylitis with respect to disease activity, pain, quality of life and response of treatment [27]. Effective treatment is available for both axial and peripheral SpA and early diagnosis and treatment are important to modify disease progression and decrease the disease burden [28]. Optimal management of SpA consists of a combination of non-pharmacological and pharmacological treatment modalities coordinated by a rheumatologist [29]. The cornerstone of non-pharmacological treatment of patients with axial SpA is patient education and regular exercise. Physiotherapy interventions have proven to be effective for ankylosing spondylitis [30]. The pharmacological treatment includes NSAIDs, diseasemodifying antirheumatic drugs (DMARDs) and anti-TNF therapy. NSAIDs are the first line drug treatment for SpA, rapidly removing pain and stiffness, While traditional NSAIDs are relatively contraindicated in patients with IBD for fear of disease exacerbation, cyclooxygenase-2 inhibitors (Coxibs) may be safe and beneficial in most patients with IBD [31]. Conventional DMARDs, which have shown to be effective for rheumatoid arthritis, have no proven effect for axial symptoms, but might be considered for peripheral symptoms. Anti-TNF therapy should be given to patients with persistently high disease activity despite conventional treatments [29]. In order to start effective treatment at an early stage, however, patients with SpA must be diagnosed early by a rheumatologist. Several studies have shown that infliximab improves the severity of spinal pain, peripheral arthritis and enthesitis in Crohn's disease [32-34]. Moreover, it has been shown that treatment of axial SpA with anti-TNF-a treatment is more effective when started in an early disease course and at a younger age $[11,12]$.

There may be several reasons why only $51 \%$ of the patients with self-reported SpA features were ever seen by a rheumatologist. First, gastroenterologists may not always spe- 
cifically ask patients with IBD about possible SpA features or do not know exactly which symptoms belong to the spectrum of SpA, The present study showed that some SpA features were significantly associated with referral to a rheumatologist (e.g. peripheral arthritis) whereas others were not (e.g. dactylitis). Second, patients may have reported symptoms in the questionnaire which they have experienced in the past, but are no longer present. If these patients were asymptomatic during their visit to the gastroenterologist, likely no referral was made. However, it is important to realize that SpA symptoms have a fluctuating course. For the diagnosis of SpA, it is not necessary to have the full range of symptoms present at the time of diagnosis, and the fluctuating character of some of the symptoms may still be an indication for referral. Third, it is possible that more patients were referred by gastroenterologists than were actually seen by a rheumatologist, due to unwillingness of patients or a visit to a rheumatologist in another hospital. Fourth, a high percentage of patients were on immunosuppressive therapy, including biologicals, which may also influence SpA symptoms. Therefore, gastroenterologists could have felt that referral to a rheumatologist would not change the management. However, we believe that every patient with possible SpA should be seen by a rheumatologist for final diagnosis and the coordination of multidisciplinary non-pharmacological and pharmacological treatment [29].

The prevalence of SpA features in patients with IBD varies widely in the literature. Any SpA manifestation was found in $17 \%$ to $62 \%$ of the patients with IBD; inflammatory back pain was found in 5\% to $30 \%$ of patients; peripheral arthritis in 5\% to 30\%; "definite" SpA classification in $12 \%$ to $46 \%$, and ankylosing spondylitis in $2 \%$ to $10 \%$ of patients with IBD [35-45]. Several factors may explain these large variations in prevalence between the different studies. First, selection of patients plays an important role. It is known that the cumulative probability of SpA increases with longer duration of IBD [46]. Hence, studies including patients with longstanding IBD will find a higher prevalence of SpA compared with studies including patients with IBD of short duration. We also found a similar trend for the diagnosis of peripheral SpA in the present study, but not for axial SpA. Second, the prevalence may vary among different ethnic populations. In a large North American Cohort of patients with IBD it was shown that Afro-American patients were more likely than Caucasians to have a diagnosis of sacroiliitis [47]. Third, the prevalence also depends on the definitions and criteria sets used. In most recent studies, classification of SpA and ankylosing spondylitis is based on the ESSG and modified NewYork criteria, respectively. With the introduction of the new ASAS criteria for axial and peripheral SpA, these criteria sets are now more frequently being used, which may lead to differences in the prevalence of the disease among studies.

Most studies published in the literature found a similar prevalence of SpA features in both CD and UC [36, 38-41, 45], although two studies showed a significantly higher prevalence of peripheral arthritis in $\operatorname{CD}[46,48]$. In our study, a trend towards more 
peripheral arthritis in CD was found, although this did not reach statistical significance. Similar to previous studies, in our study SpA features were more frequently reported in female than in male patients with IBD $[35,39]$.

Inflammatory back pain was the most frequently reported musculoskeletal SpA feature $(22.6 \%)$ in our study. In comparable studies, the prevalence of inflammatory back pain in patients with IBD ranged widely from $5 \%$ to $30 \%[36,39,41,44]$.

At least one peripheral symptom was reported by $23.7 \%$ of the patients, and a definite diagnosis of peripheral SpA was made in $5.7 \%$ of the patients. Peripheral arthritis in SpA is most frequently an asymmetric oligoarthritis of the lower limbs that is non-erosive and non-deforming, but small joint symmetrical polyarthritis or destructive lesions are also described [49]. Historically, peripheral arthritis is frequently subdivided in type 1 and type 2. Type 1 is defined as acute and self-limiting attacks of oligoarthritis that often coincide with relapses of IBD and is reported to be strongly associated with extraintestinal manifestations of IBD [44]. Type 2 is defined as a polyarthritis with symptoms persisting for months to years, running an independent course of IBD and is also associated with uveitis but not with other extraintestinal manifestations [44]. This subdivision is frequently used in gastroenterological studies, but is not used by rheumatologists in daily practice and the clinical value is probably low since much overlap exists. With the development of the new ASAS classification criteria, it is recommended to no longer use the type 1 or 2 classification, but to classify SpA into the presenting symptoms (axial and/ or peripheral), because this better reflects the need for treatment.

Some limitations of the present study need to be addressed. First, it must be emphasized that it was not the aim of the study to validate the new ASAS criteria in patients with IBD. This study was based on a self-reported questionnaire and therefore not all self-reported symptoms can automatically be interpreted as objective SpA features. Especially the inflammatory character of chronic back pain, which was reported by $22.6 \%$ of patients, is challenging. Chronic back pain of more than 3 months duration is very common in the general population, and AS accounts for no more than $5 \%$ of all patients presenting with chronic back pain [50]. An inflammatory character of the back pain is present in 70$80 \%$ of patients with AS, but also in $20-25 \%$ of the patients with mechanical back pain [51]. Therefore, not all patients reporting inflammatory back pain can be diagnosed with axial SpA. Similarly, peripheral reported symptoms of enthesitis are difficult to interpret without further evaluation. However, due to the high pre-test probability of SpA in IBD, gastroenterologists should actively ask for the presence of musculoskeletal SpA features in IBD patients, and if present, refer to a rheumatologist for further evaluation. Second, recall bias may have occurred. In this cross-sectional study patients were interviewed about possible SpA features, also in the past. Patients may have forgotten symptoms which have occurred a long time ago. Also, the majority of the patients (83.1\%) used immunosuppressive drugs, including biologicals (24.0\%), which may have influenced SpA 
symptoms. It is therefore possible that the true prevalence of SpA may be higher than currently reported. Third, a substantial proportion of the group of patients who reported symptoms were never seen by a rheumatologist. It is possible that in some patients SpA can be diagnosed. This could have influenced our final results.

In conclusion, SpA features are reported by more than one third of the patients with IBD. Review of the medical-records showed that half of the patients with self-reported SpA features were never seen by a rheumatologist, but in those who were seen in almost $80 \%$ a rheumatic disorder was being diagnosed. Treatment for SpA is more effective when started early in the disease course, therefore gastroenterologists play a key role in early recognition and referral of this often debilitating disease. 


\section{REFERENCES}

1. Greenstein AJ, Janowitz HD, Sachar DB. The extra-intestinal complications of Crohn's disease and ulcerative colitis: a study of 700 patients. Medicine 1976;55:401-12.

2. Moll JM, Haslock I, Macrae IF, et al. Associations between ankylosing spondylitis, psoriatic arthritis, Reiter's disease, the intestinal arthropathies, and Behcet's syndrome. Medicine 1974/09/01 ed1974. p. 343-64.

3. Rudwaleit M. New approaches to diagnosis and classification of axial and peripheral spondyloarthritis. Curr Opin Rheumatol 2010;22:375-80.

4. Ibn Yacoub Y, Amine B, Laatiris A, et al. Relationship between diagnosis delay and disease features in Moroccan patients with ankylosing spondylitis. Rheumatol Int 2010.

5. Davis JC, van der Heijde DM, Braun J, et al. Sustained durability and tolerability of etanercept in ankylosing spondylitis for 96 weeks. Ann Rheum Dis 2005;64:1557-62.

6. van der Heijde D, Dijkmans B, Geusens P, et al. Efficacy and safety of infliximab in patients with ankylosing spondylitis: results of a randomized, placebo-controlled trial (ASSERT). Arthritis Rheum 2005;52:582-91.

7. van der Heijde D, Kivitz A, Schiff MH, et al. Efficacy and safety of adalimumab in patients with ankylosing spondylitis: results of a multicenter, randomized, double-blind, placebo-controlled trial. Arthritis Rheum 2006;54:2136-46.

8. Antoni CE, Kavanaugh A, Kirkham B, et al. Sustained benefits of infliximab therapy for dermatologic and articular manifestations of psoriatic arthritis: results from the infliximab multinational psoriatic arthritis controlled trial (IMPACT). Arthritis Rheum 2005;52:1227-36.

9. Mease PJ, Gladman DD, Ritchlin CT, et al. Adalimumab for the treatment of patients with moderately to severely active psoriatic arthritis: results of a double-blind, randomized, placebo-controlled trial. Arthritis Rheum 2005;52:3279-89.

10. Mease PJ, Kivitz AJ, Burch FX, et al. Etanercept treatment of psoriatic arthritis: safety, efficacy, and effect on disease progression. Arthritis Rheum 2004;50:2264-72.

11. Rudwaleit M, Listing J, Brandt ], et al. Prediction of a major clinical response (BASDAI 50) to tumour necrosis factor alpha blockers in ankylosing spondylitis. Ann Rheum Dis 2004;63:665-70.

12. Rudwaleit M, Schwarzlose S, Hilgert ES, et al. MRI in predicting a major clinical response to anti-tumour necrosis factor treatment in ankylosing spondylitis. Ann Rheum Dis 2008;67:1276-81.

13. van der Linden S, Valkenburg HA, Cats A. Evaluation of diagnostic criteria for ankylosing spondylitis. A proposal for modification of the New York criteria. Arthritis Rheum 1984;27:361-8.

14. Mau W, Zeidler H, Mau R, et al. Clinical features and prognosis of patients with possible ankylosing spondylitis. Results of a 10-year followup. J Rheumatol 1988;15:1109-14.

15. Dougados M, van der Linden S, Juhlin R, et al. The European Spondylarthropathy Study Group preliminary criteria for the classification of spondylarthropathy. Arthritis Rheum 1991;34:1218-27.

16. Amor B, Dougados M, Mijiyawa M. [Criteria of the classification of spondylarthropathies]. Rev Rhum Mal Osteoartic 1990;57:85-9.

17. Baddoura R, Awada H, Okais J, et al. Validation of the European Spondylarthropathy Study Group and B. Amor criteria for spondylarthropathies in Lebanon. Rev Rhum Engl Ed 1997;64:459-64.

18. Erturk M, Alaca R, Tosun E, et al. Evaluation of the Amor and ESSG classification criteria for spondylarthropathies in a Turkish population. Rev Rhum Engl Ed 1997;64:293-300.

19. Collantes-Estevez E, Cisnal del Mazo A, Munoz-Gomariz E. Assessment of 2 systems of spondyloarthropathy diagnostic and classification criteria (Amor and ESSG) by a Spanish multicenter study. European Spondyloarthropathy Study Group. J Rheumatol 1995;22:246-51.

20. Collantes E, Veroz R, Escudero A, et al. Can some cases of 'possible' spondyloarthropathy be classified as 'definite' or 'undifferentiated' spondyloarthropathy? Value of criteria for spondyloarthropathies. Spanish Spondyloarthropathy Study Group. Joint Bone Spine 2000;67:516-20.

21. Rudwaleit M, van der Heijde D, Landewe R, et al. The development of Assessment of SpondyloArthritis international Society classification criteria for axial spondyloarthritis (part II): validation and final selection. Ann Rheum Dis 2009;68:777-83. 
22. Rudwaleit M, van der Heijde D, Landewe R, et al. The Assessment of SpondyloArthritis International Society classification criteria for peripheral spondyloarthritis and for spondyloarthritis in general. Ann Rheum Dis 2011;70:25-31.

23. Harvey RF, Bradshaw JM. A simple index of Crohn's-disease activity. Lancet 1980;1:514.

24. Walmsley RS, Ayres RC, Pounder RE, et al. A simple clinical colitis activity index. Gut 1998;43:29-32.

25. Boonen A, van der Linden SM. The burden of ankylosing spondylitis. J Rheumatol Suppl 2006;78:4-11.

26. Landewe $\mathrm{R}$, Dougados $\mathrm{M}$, Mielants $\mathrm{H}$, et al. Physical function in ankylosing spondylitis is independently determined by both disease activity and radiographic damage of the spine. Ann Rheum Dis 2009;68: 863-7.

27. Rudwaleit M, Haibel H, Baraliakos $X$, et al. The early disease stage in axial spondylarthritis: results from the German Spondyloarthritis Inception Cohort. Arthritis Rheum 2009;60:717-27.

28. Sieper ], Rudwaleit M, Khan MA, et al. Concepts and epidemiology of spondyloarthritis. Best Pract Res Clin Rheumatol 2006;20:401-17.

29. Braun J, van den Berg R, Baraliakos X, et al. 2010 update of the ASAS/EULAR recommendations for the management of ankylosing spondylitis. Ann Rheum Dis 2011;70:896-904.

30. Dagfinrud H, Kvien TK, Hagen KB. Physiotherapy interventions for ankylosing spondylitis. Cochrane Database Syst Rev 2008;23.

31. El Miedany Y, Youssef S, Ahmed I, et al. The gastrointestinal safety and effect on disease activity of etoricoxib, a selective cox-2 inhibitor in inflammatory bowel diseases. Am ] Gastroenterol 2006;101:311-7.

32. Generini S, Giacomelli R, Fedi R, et al. Infliximab in spondyloarthropathy associated with Crohn's disease: an open study on the efficacy of inducing and maintaining remission of musculoskeletal and gut manifestations. Ann Rheum Dis 2004;63:1664-9.

33. Herfarth $\mathrm{H}$, Obermeier $\mathrm{F}$, Andus $\mathrm{T}$, et al. Improvement of arthritis and arthralgia after treatment with infliximab (Remicade) in a German prospective, open-label, multicenter trial in refractory Crohn's disease. Am J Gastroenterol 2002;97:2688-90.

34. Van den Bosch F, Kruithof E, De Vos M, et al. Crohn's disease associated with spondyloarthropathy: effect of TNF-alpha blockade with infliximab on articular symptoms. Lancet 2000;356:1821-2.

35. Beslek A, Onen F, Birlik M, et al. Prevalence of spondyloarthritis in Turkish patients with inflammatory bowel disease. Rheumatol Int 2009;29:955-7.

36. de Vlam K, Mielants $\mathrm{H}$, Cuvelier $\mathrm{C}$, et al. Spondyloarthropathy is underestimated in inflammatory bowel disease: prevalence and HLA association. J Rheumatol 2000;27:2860-5.

37. Palm O, Moum B, Ongre A, et al. Prevalence of ankylosing spondylitis and other spondyloarthropathies among patients with inflammatory bowel disease: a population study (the IBSEN study). J Rheumatol 2002;29:511-5.

38. Rodriguez VE, Costas PJ, Vazquez M, et al. Prevalence of spondyloarthropathy in Puerto Rican patients with inflammatory bowel disease. Ethn Dis 2008;18:S2-225-9.

39. Salvarani C, Vlachonikolis IG, van der Heijde DM, et al. Musculoskeletal manifestations in a populationbased cohort of inflammatory bowel disease patients. Scand ] Gastroenterol 2001;36:1307-13.

40. Turkcapar N, Toruner M, Soykan I, et al. The prevalence of extraintestinal manifestations and HLA association in patients with inflammatory bowel disease. Rheumatol Int 2006;26:663-8.

41. Lanna CC, Ferrari Mde L, Rocha SL, et al. A cross-sectional study of 130 Brazilian patients with Crohn's disease and ulcerative colitis: analysis of articular and ophthalmologic manifestations. Clin Rheumatol 2008;27:503-9.

42. Protzer U, Duchmann R, Hohler $T$, et al. Enteropathic spondylarthritis in chronic inflammatory bowel diseases: prevalence, manifestation pattern and HLA association. Med Klin (Munich) 1996:91:330-5.

43. Scarpa R, del Puente A, D'Arienzo A, et al. The arthritis of ulcerative colitis: clinical and genetic aspects. J Rheumatol 1992;19:373-7.

44. Orchard TR, Wordsworth BP, Jewell DP. Peripheral arthropathies in inflammatory bowel disease: their articular distribution and natural history. Gut 1998:42:387-91. 
45. Palm O, Moum B, Jahnsen J, et al. The prevalence and incidence of peripheral arthritis in patients with inflammatory bowel disease, a prospective population-based study (the IBSEN study). Rheumatology (Oxford) 2001;40:1256-61.

46. Veloso FT, Carvalho ], Magro F. Immune-related systemic manifestations of inflammatory bowel disease. A prospective study of 792 patients. ] Clin Gastroenterol 1996;23:29-34.

47. Nguyen GC, Torres EA, Regueiro M, et al. Inflammatory bowel disease characteristics among African Americans, Hispanics, and non-Hispanic Whites: characterization of a large North American cohort. Am J Gastroenterol 2006;101:1012-23.

48. Mendoza JL, Lana R, Taxonera C, et al. [Extraintestinal manifestations in inflammatory bowel disease: differences between Crohn's disease and ulcerative colitis]. Med Clin (Barc) 2005;125:297-300.

49. Mielants H, Veys EM, Goethals K, et al. Destructive lesions of small joints in seronegative spondylarthropathies: relation to gut inflammation. Clin Exp Rheumatol 1990;8:23-7.

50. Underwood MR, Dawes P. Inflammatory back pain in primary care. British journal of rheumatology 1995; 34:1074-7.

51. Rudwaleit M, van der Heijde $D$, Khan MA, et al. How to diagnose axial spondyloarthritis early. Annals of the rheumatic diseases 2004;63:535-4 




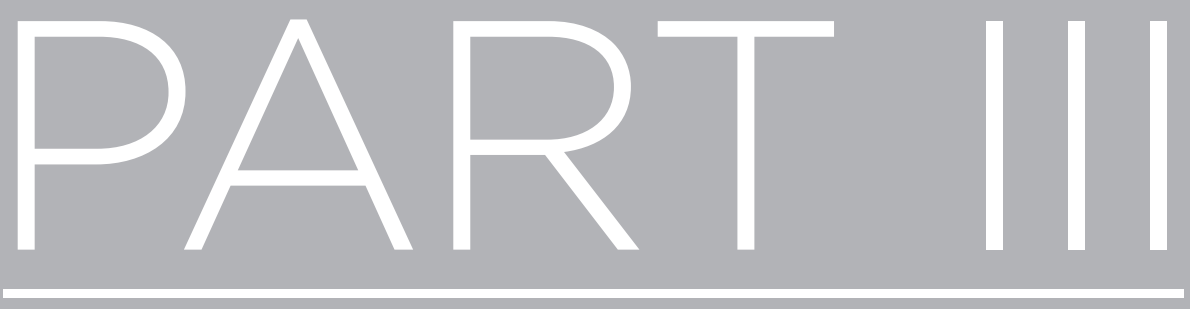

\section{Comorbidities in ankylosing spondylitis}

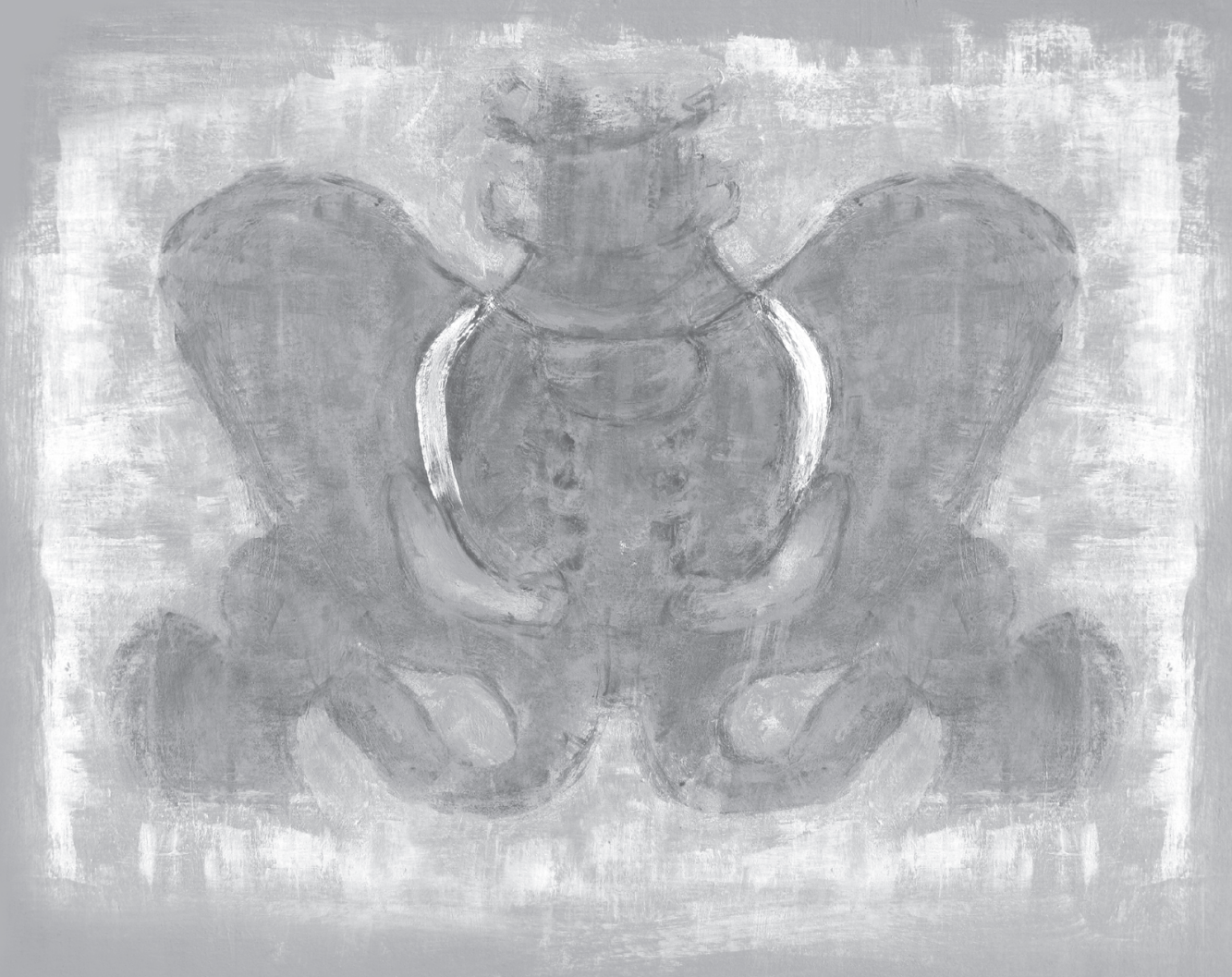





\section{CHAPTER 6 \\ Aspects of validity of the self-administered comorbidity questionnaire (SCO) in patients with ankylosing spondylitis}

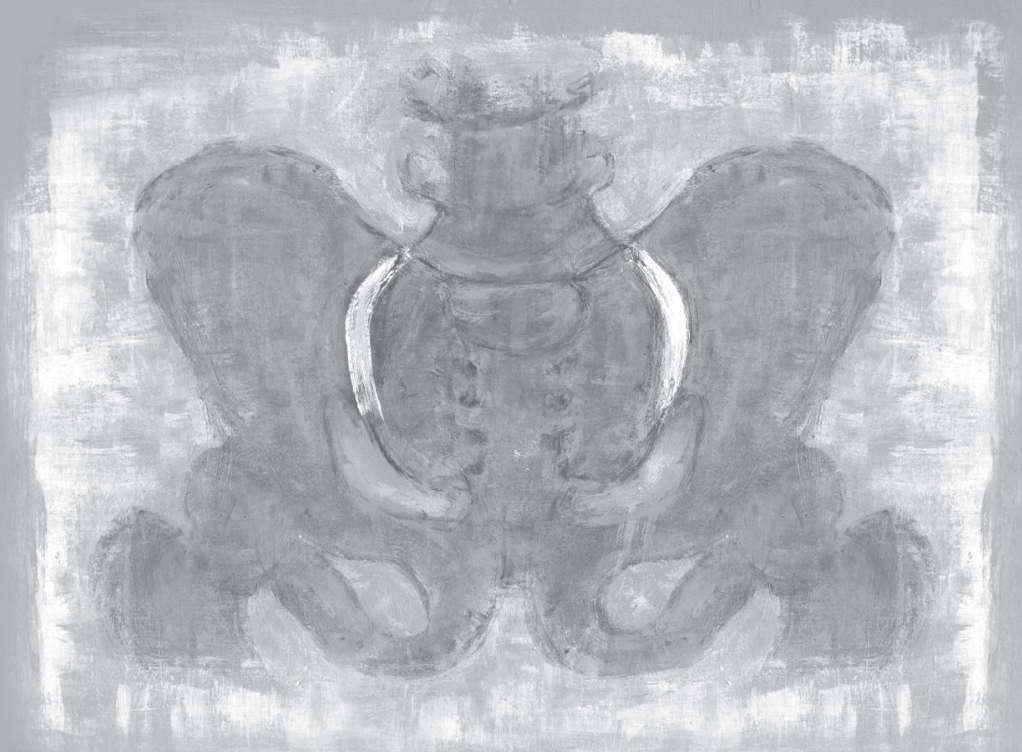

Carmen Stolwijk, Astrid van Tubergen, Sofia Ramiro, Ivette Essers, Marc Blaauw, Désirée van der Heijde, Robert Landewé, Filip van den Bosch, Maxime Dougados, Annelies Boonen 


\section{ABSTRACT}

\section{Objectives}

To evaluate criterion and construct validity of the self-administered comorbidity questionnaire (SCO) in patients with ankylosing spondylitis (AS).

\section{Methods}

The SCQ and indices of disease activity, physical function, health-related quality of life (HROLL) and work disability were administered to 98 patients with AS. Criterion validity was assessed by the agreement between the SCQ answers and comorbidities identified in medical records. Construct validity was assessed by correlating the SCO with the Charlson-index and Michaud/Wolfe-index; by correlating the SCO with demographics, physical function, HROoL and AS-related disease activity; and by exploring the contribution of comorbidity to these outcomes while adjusting for clinical-demographic characteristics. Furthermore, a modified version of the SCO (mSCQ) was evaluated for the same aspects of validity, after removing rheumatic conditions.

\section{Results}

Agreement was moderate to perfect for most conditions (kappa 0.47-1.00), except for ulcer disease, depression and osteoarthritis (kappa 0.14-0.15). The correlation between the SCQ and Charlson- and Michaud/Wolfe-index was 0.24 and 0.39 respectively, and between the mSCO and both indices 0.36 and 0.53. Both SCO and mSCO correlated weakly to moderately with age, physical function and HROOL (0.24-0.45). The SCO also correlated weakly with disease activity (0.27) while the mSCO did not (0.17). In multivariable analysis, both SCO and $\mathrm{mSCO}$ contributed independently to physical function, HROoL and work disability, while the Michaud/Wolfe and Charlson-index did not.

\section{Conclusion}

The SCO is a promising instrument to determine comorbidities and to understand the impact on health outcomes in patients with AS. Excluding rheumatic conditions from the SCO (mSCO) improved validity. 


\section{INTRODUCTION}

Ankylosing spondylitis (AS) is a chronic inflammatory disease of the sacroiliac joints and spine that can lead to substantial functional limitations [1-4]. In addition to axial and peripheral articular manifestations, extra-articular manifestations related to the concept of spondyloarthritis (SpA) comprising uveitis, psoriasis and inflammatory bowel disease (IBD), occur frequently and contribute to the burden of disease. Moreover, patients with AS can also develop comorbidities, which are also seen in the general population. It is increasingly recognized that some of these comorbidities, including cardiovascular disease and osteoporosis, occur more frequently in patients with AS than in the general population, probably as a result of the inflammatory pathophysiological process [5-8]. It has been recognized that comorbidities can affect the detection, prognosis, therapy but also outcome of a single condition at any moment [9]. As a consequence, adjustment for comorbidity is important for outcome studies which aim at exploring or predicting different aspects of health-related quality of life (HROLL), work participation, health services utilization or death [10-14].

Several instruments have been developed to identify and measure comorbidities for use in statistical analyses [15-17]. The instruments differ in their purposes and approach of measurement. Instruments can be generic or disease specific, can be used at individual patient level or for clinical research, can focus on different outcomes of interest (mortality vs. health status), can rely on different approaches to assess comorbidities (self-reported vs. medical-record based) and can have different approaches of weighting the individual comorbidities into a final score. In AS, literature on the role of comorbidities on patient outcomes is scarce. Notwithstanding, available studies showed that comorbidity contributes to physical function, HRQoL and work participation outcomes $[2,18,19]$. Therefore, comorbidity should be considered as a confounder, effect-modifier or predictor in studies on outcomes in AS. However, no comorbidity instrument has been evaluated for use in outcome studies in AS, hampering the influence of comorbidities on health status.

The self-administered comorbidity questionnaire (SCO) is a generic, self-report questionnaire with 13 common medical conditions, developed to adjust for the impact of comorbidity on functional status [17]. The SCO was validated in 170 inpatients from medical and surgical care units and was found to be an efficient instrument to assess comorbid conditions in clinical and health services research among patients with a broad range of the underlying diseases [17]. The clinimetric properties of the SCO have also been validated in some rheumatic diseases $[20,21]$. In patients with $A S$, however, the SCO has been used as an instrument to measure comorbidity [2], but aspects of validity have never been evaluated.

The aim of the present study was to assess criterion and construct validity of the SCO in a prevalence cohort of patients with AS. We hypothesized that a valid comorbidity 
index, which aims to adjust health status for the influence of comorbidity, should have the following properties: capture similar comorbidities as documented in the medical records (criterion validity); correlate with other existing measures of comorbidity, in particular with those relating comorbidities to health status (construct validity); correlate with age and to some extent with HRQoL, physical function and work participation, but less with AS-related disease activity (construct validity); and behave as an independent contributor, confounder or effect-modifier on physical function, HROLL and work disability (construct validity).

\section{PATIENTS AND METHODS}

\section{Patients}

For the present study, data from the Outcome in Ankylosing Spondylitis International Study (OASIS) were used [22]. OASIS is a longitudinal observational prevalence cohort which started in 1996 and included 217 consecutive outpatients with AS (according to the modified New York criteria) [23] from the Netherlands ( $n=137)$, Belgium $(n=25)$ and France $(n=55)$, without further eligibility criteria. To date, patients have been followed for up to 12 years and are being assessed regularly by questionnaires, clinimetry, laboratory assessments and radiography. Before each study visit, a postal questionnaire including all instruments was sent to the patients' homes. The patients returned the questionnaires to the researchers at the study visit and, if applicable, patients were asked to complete missing items. In year 8 of follow-up (year 2005), the SCO was added to the questionnaire. For the present analysis, the SCO data of patients from the Netherlands $(n=87)$ and Belgium $(n=11)$ that were still participating were used. French patients were excluded, because no medical records outside the rheumatology department could be retrieved to assess agreement between SCQ responses and medical record diagnosis. The OASIS study was approved by the hospital ethics committee and informed consent was obtained from all subjects.

\section{Study instrument}

The SCO asks patients to indicate if they suffer at this moment from 13 additional medical conditions that might have impact on functioning [17]. The conditions are: heart disease, high blood pressure, lung disease, diabetes, ulcer or stomach disease, kidney disease, liver disease, anemia or other blood disease, cancer, depression, osteoarthritis, back pain, rheumatoid arthritis (in the Dutch version this was translated as 'chronic rheumatic disease') and an option to add three other non-specified medical problems. Osteoarthritis and rheumatoid arthritis are listed separately, but are scored as one. The patient is asked to indicate for each condition if it is present (yes/no), is currently treated 
(yes/no) and/or imposes functional limitations (yes/no). Every 'yes' is given one point contributing to a maximum score of 45 . For the present study, a modified version of the SCO (mSCO) was also evaluated by removing the items related to rheumatic conditions (chronic rheumatic disease, back pain and osteoarthritis), because these conditions were assumed to be difficult to distinguish from the index disease (AS) and because patients likely cannot separate the functional impact of these diseases from the impact of AS. The mSCO is scored similarly as the SCO, but due to fewer items the score ranges from 0 to 39.

\section{Criterion validity}

Criterion validity is the degree to which the scores of an instrument are an adequate reflection of the 'gold standard' [24]. We studied this by evaluating the agreement between the self-reported comorbidities and current treatment with data from the medical records.

The comorbidity and treatment data were extracted from the medical records by two independent chart extractors, who were blinded to the SCO data, using a predefined list of comorbidities. The comorbidities were extracted for each year between 1996 and 2005, or were considered as 'present before 1996'. A condition was considered as present when any physician diagnosis or disease specific medication was noted in the record (e.g. diabetes was identified as present when insulin was noted). Reports from x-rays were checked on the presence of osteoarthritis (both of the spine and peripheral joints) and osteoarthritis was also scored as present if the radiologist clearly described it in the report. Each of the extractors dealt with about half the sample. To assess intra- and interobserver reliability of the data acquisition, each chart extractor reviewed both a random sample of 10 charts a second time with a one month interval and a random sample of 10 charts previously reviewed by the other extractor.

\section{Construct validity}

Construct validity is the degree to which the scores of an instrument are consistent with hypotheses, e.g. with regard to positive (convergent validity) or absent relationships (discriminant validity) with scores of other instruments [24].

First, we hypothesized that the SCO should correlate with other existing measures of comorbidity: the Charlson index and the Michaud/Wolfe index (convergent validity). The Charlson index (range 0 to 33) is the most extensively applied record-based comorbidity index and was developed to predict mortality [15]. The Charlson index consists of 19 conditions with specific weighing for each condition based on the mortality-risk. The Michaud/Wolfe index (range 0 to 9 ) is originally a self-reported comorbidity index including 11 weighted conditions and was developed for outpatients with four different rheumatic diseases (rheumatoid arthritis, osteoarthritis, systemic lupus erythematous, 
fibromyalgia) to predict several outcomes including functional status and health resource utilization [25]. For the present study, both the Charlson- and the Michaud/Wolfe index were calculated using data retrieved from the medical records. Intra- and inter-observer reliability of the chart extractors to calculate these scores was assessed.

Second, we hypothesized that the SCO should correlate with age, at least weakly with generic as well as AS-specific HROoL, physical function and work disability, but not with AS-related disease activity, because the index is meant to measure diseases other than the index disease (AS). Physical function was measured with the Bath AS Functional Index (BASFI) [26]. Generic HROoL was measured with the 36-item Short-Form (SF-36) health survey questionnaire (Dutch RAND-36 version 1.0) [27] of which the SF-36 physical component summary score (SF-36 PCS) and the SF-36 mental component summary score (SF-36 MCS) were calculated using norm-based data from the Dutch and US population (mean 50, SD 10) $[28,29]$. AS-specific HRQoL was measured with the AS Quality of Life (ASOLL) [30]. Disease activity was measured with the Bath AS Disease Activity Index (BASDAI) [31], the AS Disease Activity Score (ASDAS) [32], inflammatory parameters (CRP, ESR), and both the patient and physician global assessment score. Work status was included in the demographic questions and asked whether patients had withdrawn from labour force due to (partial) work disability. To compare the SCO with medical-record based comorbidity indices, correlations with all constructs were also calculated for the Charlson- and Michaud/Wolfe index.

Third, we hypothesized that comorbidity as measured with the SCO should behave as an independent contributor, confounder or effect-modifier on physical function (BASFI), HROOL (SF-36 PCS) and work disability. This was tested by computing the relative contribution of the SCQ to these outcomes in a multivariable regression analysis while adjusting for clinical-demographic characteristics. For comparison, the same analyses were repeated for the Charlson- and Michaud/Wolfe index.

\section{Statistical analysis}

Descriptive statistics were used to describe demographic data and the frequency of reported comorbidities in the SCO and in the medical records.

Criterion validity. Agreement between the patients' answers on the presence and treatment of the condition in the SCO with the comorbidities and treatment retrieved from the medical records was calculated with kappa statistics. For conditions with a prevalence of $0 \%$ or $100 \%$ documented by either SCQ or medical records, kappa statistics could not be calculated. Kappa-values of 0-0.20 indicate slight agreement; 0.21-0.40, fair agreement; 0.41-0.60, moderate agreement; 0.61-0.80, substantial agreement; and 0.81-1.0, almost perfect agreement [33]. As part of the criterion validity, the intra- and inter-observer reliability of the data acquisition from the medical records was explored 
by calculating kappa statistics for the items 'condition present' and 'condition treated' for each condition of the SCO.

Construct validity. The correlations between the SCQ, the mSCO and continuous outcomes were evaluated using Spearman's correlation coefficients and the association between comorbidity and work disability was calculated using odds ratios (OR) (95\% $\mathrm{Cl}$ ). Correlation coefficients of 0.21-0.40 were qualified as weak, 0.41-0.75 as moderate, and above 0.75 as strong [9]. As part of the convergent validity, the intra- and interobserver reliability of the data retrieved by the chart extractors used for computing the Charlson- and Michaud/Wolfe index was assessed with two-way mixed model intraclass correlation coefficients (ICC) (absolute agreement, single measures). A value of $>0.60$ represents good reliability; >0.8, very good reliability; and $>0.90$, excellent reliability. The relative contribution of the SCO and $\mathrm{mSCO}$ to variations in physical function (BASFI), HROLL (SF-36 PCS) and work disability was tested in univariable followed by multivariable regression analysis while controlling for clinical-demographic (age, sex, body-mass index (BMI), higher education) and disease characteristics (symptom duration, BASDAI, BASMI, BASFI), if appropriate. Variables with p-values $<0.10$ in the univariable analysis were chosen as candidate variables for multivariable regression analysis (forward regression method). The comorbidity score was entered first and kept in the model. The other variables were entered (if $p<0.10$ in univariable analysis) and removed (if $p>0.05$ in multivariable analysis) hierarchically. All possible interactions between the variables were tested in separate analyses. The same analyses were repeated for the Charlson- and Michaud/Wolfe index. Statistical analyses were performed using SPSS 18.0.

\section{RESULTS}

\section{Patient characteristics}

All 98 patients completed all items of the SCO. Characteristics of the patients are presented in table 1 . The sample was similar to the 217 patients included in OASIS at baseline in terms of age, gender, symptom- and disease duration, disease activity and function (data not shown) [22]. The mean SCO-score was 5.4 (SD 4.1) and the mean score for the mSCQ was 2.9 (SD 3.0). Table 2 shows the patients' responses on the SCQ and the frequency of comorbidities extracted from the medical records. Eighty-seven (88.8\%) patients reported at least one comorbidity, of which 64 (65.3\%) patients reported at least one non-rheumatic condition. With respect to the rheumatic conditions, 67 (68.4\%) patients reported to have back pain, 19 (19.4\%) patients reported osteoarthritis and 15 $(15.3 \%)$ patients reported chronic rheumatic disease. Within the last group, 11 (73.3\%) patients had a history of peripheral arthritis, compared to 48 of 83 (57.8\%) patients who indicated not to suffer from a chronic rheumatic disease $(p=0.26)$. At least one additional 
Table 1. Characteristics of study sample $(n=98)$

\begin{tabular}{|c|c|}
\hline Characteristic & Value \\
\hline Age, years & $53.9(11.4)$ \\
\hline Men, $n(\%)$ & $69(70.4 \%)$ \\
\hline Dutch/Belgian, $n(\%)$ & $87 / 11(88.8 / 11.2 \%)$ \\
\hline Duration since diagnosis, years & $20.6(9.4)$ \\
\hline Duration of complaints, years & $31.2(11.5)$ \\
\hline HLA-B27 positive/negative/missing, $n(\%)$ & $82 / 13 / 3(84 / 13 / 3 \%)$ \\
\hline $\mathrm{BMI}, \mathrm{kg} / \mathrm{m}^{2}$ & $26.1(4.4)$ \\
\hline Working, $n(\%)^{a}$ & $37(44.6 \%)$ \\
\hline Full or partial work disability, $n(\%)^{a}$ & $43(51.8 \%)$ \\
\hline Higher education, $n(\%)^{\text {a }}$ & $13(15.7 \%)$ \\
\hline BASDAI $(0-10)$ & $3.8(2.2)$ \\
\hline ASDAS-CRP & $2.5(1.0)$ \\
\hline BASFI (0 - 10) & $4.2(2.5)$ \\
\hline SF-36 Physical component summary score (0 - 100) & $39.2(11.5)$ \\
\hline SF-36 Mental component summary score (0 - 100) & $50.0(12.4)$ \\
\hline ASOOL $(0$ - 18) & $6.6(4.6)$ \\
\hline Patient global $(0-10)$ & $4.0(2.6)$ \\
\hline Physician global (0 - 10) & $3.8(2.5)$ \\
\hline History of uveitis, $n(\%)$ & $50(51.1 \%)$ \\
\hline History of inflammatory bowel disease, $n(\%)$ & $22(22.4 \%)$ \\
\hline History of psoriasis, $n(\%)$ & $11(11.2 \%)$ \\
\hline ESR, mm/hour, median [IQR] & $14[5-24]$ \\
\hline CRP, mg/l, median [IOR] & $5.7[2-11]$ \\
\hline SCO $(0-45)$, mean (SD), median [IOR] & $5.4(4.1), 5[2-8]$ \\
\hline mSCQ (0-39), mean (SD), median [IOR] & $2.9(3.0), 2[0-5]$ \\
\hline Charlson index (0-33), mean (SD), median [IOQR] & $0.3(0.7), 0[0-0]$ \\
\hline Michaud/Wolfe index (0-9), mean (SD), median [IOR] & $1.1(1.5), 1[0-2]$ \\
\hline
\end{tabular}

Numbers are expressed as mean (standard deviation (SD)), unless otherwise stated. a \% only of patients < 65 years of age $(n=83)$. BMI: Body-Mass Index; BASDAl: Bath Ankylosing Spondylitis Disease Activity Index; ASDAS-CRP: Ankylosing Spondylitis Disease Activity Score (C-reactive protein); BASFI: Bath Ankylosing Spondylitis Functional Index; SF-36: Short Form-36; ASO-L: Ankylosing Spondylitis Quality of Life; ESR: erythrocyte sedimentation rate; CRP: C-reactive protein; SCO: self-administered comorbidity questionnaire; mSCQ: modified self-administered comorbidity questionnaire; IQR: Interquartile range.

condition was reported by $43(43.9 \%)$ patients and included, among others, IBD ( $n=10)$, uveitis $(n=5)$, neurologic diseases $(n=7)$ and psoriasis $(n=1)$.

\section{Criterion validity}

Table 2 also shows the agreement ( $\mathrm{k}$ ) between self-reported presence and treatment of each comorbidity in the SCQ with presence and treatment of this comorbidity retrieved 
Table 2. Prevalence of comorbid conditions and agreement between SCQ and medical-records $(n=98)$

\begin{tabular}{|c|c|c|c|c|c|c|c|}
\hline \multirow[b]{2}{*}{ Disease } & \multicolumn{3}{|c|}{ SCO } & \multicolumn{2}{|c|}{ Medical records } & \multicolumn{2}{|c|}{ Карра (95\% CI) } \\
\hline & $\begin{array}{c}\text { Present, } \\
n(\%)\end{array}$ & $\begin{array}{c}\text { Treatment, } \\
\mathrm{n} \text { (\% of present) }\end{array}$ & $\begin{array}{c}\text { Limitations, } \\
\text { n (\% of present) }\end{array}$ & $\begin{array}{l}\text { Present, } \\
n(\%)\end{array}$ & $\begin{array}{c}\text { Treatment, } \\
\mathrm{n} \text { (\% of present) }\end{array}$ & Presence & Treatment \\
\hline Heart disease & $4(4.1)$ & $3(75.0)$ & $3(75.0)$ & $4(4.1)$ & $4(100)$ & $0.88(0.65-1.0)$ & $0.85(0.56-1.0)$ \\
\hline Hypertension & $27(27.6)$ & $21(77.8)$ & $2(7.4)$ & $27(27.6)$ & $27(100)$ & $0.69(0.53-0.85)$ & $0.67(0.50-0.84)$ \\
\hline Lung disease & $6(6.1)$ & $4(66.7)$ & $5(83.3)$ & $6(6.1)$ & $5(83.3)$ & $0.47(0.11-0.83)$ & $0.42(0.01-0.84)$ \\
\hline Diabetes & $4(4.1)$ & $2(50.0)$ & 0 & $4(4.1)$ & $4(100)$ & $0.74(0.39-1.0)$ & $0.66(0.22-1.0)$ \\
\hline $\begin{array}{l}\text { Ulcer/stomach } \\
\text { disease }\end{array}$ & $8(8.2)$ & $7(87.5)$ & $3(37.5)$ & $3(3.1)$ & $3(100)$ & $0.14(0.0-0.45)$ & $0.16(0.0-0.50)$ \\
\hline Kidney disease & $4(4.1)$ & $2(50.0)$ & $1(25.0)$ & $2(2.0)$ & $0(0)$ & $0.66(0.22-1.0)$ & NC \\
\hline Liver disease & 0 & 0 & 0 & 0 & 0 & NC & NC \\
\hline $\begin{array}{l}\text { Anemia/other } \\
\text { blood disease }\end{array}$ & $6(6.1)$ & $5(83.3)$ & $2(33.3)$ & $3(3.1)$ & $2(66.7)$ & $0.65(0.29-1.0)$ & $0.56(0.12-1.0)$ \\
\hline Cancer & $1(1.0)$ & 0 & 0 & $1(1.0)$ & $0(0)$ & $1.00(1.0)$ & NC \\
\hline Depression & $9(9.2)$ & $4(44.4)$ & $5(55.5)$ & $2(2.0)$ & $2(100)$ & $0.15(0.0-0.45)$ & 0.0 \\
\hline Osteoarthritis & $19(19.4)$ & $12(63.2)$ & $11(57.9)$ & $27(27.6)$ & $25(92.6)$ & $0.14(0.0-0.36)$ & $0.13(0.0-0.33)$ \\
\hline Back pain & $67(68.4)$ & $52(77.6)$ & $53(79.1)$ & $98(100)$ & NR & NC & NC \\
\hline $\begin{array}{l}\text { Chronic rheumatic } \\
\text { disease }\end{array}$ & 15 (15.3) & $12(80.0)$ & $11(73.3)$ & $98(100)$ & NR & NC & NC \\
\hline Additional 1 & $43(43.9)$ & $35(81.4)$ & $27(62.8)$ & NR & NR & NC & NC \\
\hline Additional 2 & $12(12.2)$ & $10(83.3)$ & 11 (91.7) & NR & NR & NC & NC \\
\hline Additional 3 & $4(4.1)$ & $3(75.0)$ & $4(100)$ & NR & NR & NC & NC \\
\hline
\end{tabular}

NC: not calculable (prevalence was $0 \%$ or $100 \%$ or not recorded); NR: not recorded. SCQ: selfadministered comorbidity questionnaire.

from the medical records. The agreement varied from slight (depression, ulcer or stomach disease and osteoarthritis) to substantial or perfect agreement (heart disease, hypertension, diabetes, kidney-disease, anemia and cancer). Kappa-values could not be calculated for back pain and chronic rheumatic disease, because the prevalence was $100 \%$ in this study among patients with AS.

The reliability analyses of the data acquisition from the medical records showed that both the intra- and inter-observer reliability for the specific items in the SCO was substantial to almost perfect (kappa 0.64-1.00), except for osteoarthritis (kappa 0.26 and 0.49 for intra- and inter-rater reliability, respectively).

\section{Construct validity}

Table 3 shows the correlations of the SCO and mSCO with the Charlson index and Michaud/Wolfe index. The correlations between the instruments were low to moderate (0.24-0.57), but better for the $\mathrm{MSCO}$ compared with the SCO and better with the Michaud/Wolfe index compared with the Charlson index. The intra- and inter-observer 
Table 3. Construct validity of the SCO, mSCO, Charlson index and Michaud/Wolfe index with clinical and laboratory measures

\begin{tabular}{|c|c|c|c|c|}
\hline & SCQ & mSCQ & Charlson-index & Michaud/Wolfe-index \\
\hline SCO $(n=98)$ & - & $0.86^{*}$ & $0.24 *$ & $0.43^{*}$ \\
\hline $\mathrm{mSCO}(n=98)$ & - & - & $0.36 *$ & $0.57^{*}$ \\
\hline Charlson-index (n=98) & - & - & - & $0.61 *$ \\
\hline Michaud/Wolfe-index ( $n=98)$ & - & - & - & - \\
\hline Age $(n=98)$ & $0.24^{*}$ & $0.28 *$ & $0.30 \%$ & $0.46 *$ \\
\hline BASDAI $(n=96)$ & $0.27^{*}$ & 0.17 & -0.01 & 0.03 \\
\hline ASDAS-CRP $(n=86)$ & 0.18 & 0.16 & 0.13 & 0.09 \\
\hline BASFI $(n=98)$ & $0.43^{*}$ & $0.41^{*}$ & $0.22^{*}$ & $0.31 *$ \\
\hline SF-36 physical ( $n=98)$ & $-0.45^{*}$ & $-0.42 *$ & -0.17 & -0.26 \\
\hline SF-36 mental $(n=98)$ & -0.10 & -0.02 & 0.09 & 0.18 \\
\hline ASOOL (n=98) & $0.43^{*}$ & $0.32 *$ & 0.11 & 0.12 \\
\hline Patient global (n=98) & $0.22^{*}$ & 0.19 & -0.07 & 0.04 \\
\hline Physician global (n=89) & $0.35 *$ & $0.23^{*}$ & -0.09 & 0.13 \\
\hline $\operatorname{ESR}(n=83)$ & -0.05 & 0.12 & 0.18 & 0.13 \\
\hline $\operatorname{CRP}(n=87)$ & -0.03 & 0.07 & 0.22 & 0.18 \\
\hline Work disability [OR; $95 \% \mathrm{Cl}]^{a}(\mathrm{n}=98)$ & $1.31(1.13-1.53)$ & $1.48(1.20-1.81)$ & $0.00(0.00)$ & $2.57(1.41-4.69)$ \\
\hline
\end{tabular}

Data are expressed as Spearman correlation coefficients, except for work disability (odd's ratio (OR)), *statistically significant, a only in patients $<65$ years.

SCQ: self-administered comorbidity questionnaire; mSCQ: modified self-administered comorbidity questionnaire; BASDAl: Bath Ankylosing Spondylitis Disease Activity Index; ASDAS-CRP: Ankylosing Spondylitis Disease Activity Score (C-reactive protein); BASFI: Bath Ankylosing Spondylitis Functional Index; SF-36: Short Form-36; ASOLL: Ankylosing Spondylitis Quality of Life; ESR: erythrocyte sedimentation rate; CRP: C-reactive protein.

reliability of data acquisition to calculate the Charlson- and Michaud/Wolfe index was very good $(0.85,95 \% \mathrm{Cl} 0.65-0.94)$ to excellent $(0.91,95 \% \mathrm{Cl} 0.78-0.96)$.

Table 3 also shows the correlations with the other constructs. All comorbidity indices correlated with age. The SCO and mSCO correlated both moderately with HROoL and physical function, whereas for the Charlson- and Michaud/Wolfe index only weak correlations were found. The SCQ correlated weakly with some measures of disease activity, whereas the mSCO did not. Strong associations were found between SCQ, mSCQ and the Michaud/Wolfe index with work disability.

The results of the regression analyses are shown in tables 4, 5 and 6. Assumptions of homoscedasticity and linearity were checked and were met. All four comorbidity indices were in univariable analysis significantly associated with the BASFI, but only the SCO and mSCO remained significant in multivariable analysis. The SCO, mSCO and Michaud/Wolfe index were associated with the SF-36 PCS in univariable analysis, but in multivariable analysis, only SCO and mSCO significantly contributed to the SF-36 PCS. The SCO, mSCO and Michaud/Wolfe index were associated with work disability in univariable analysis, 


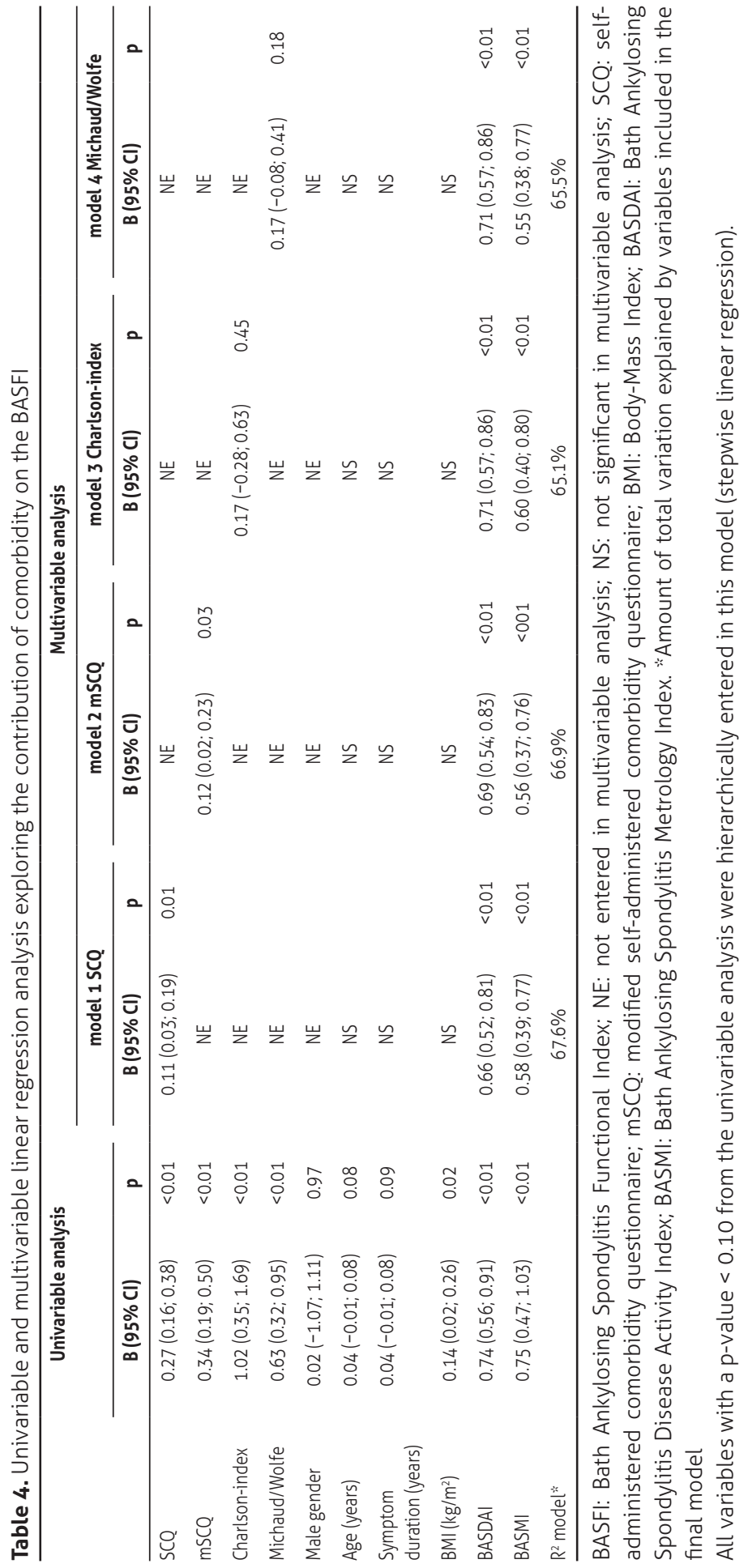




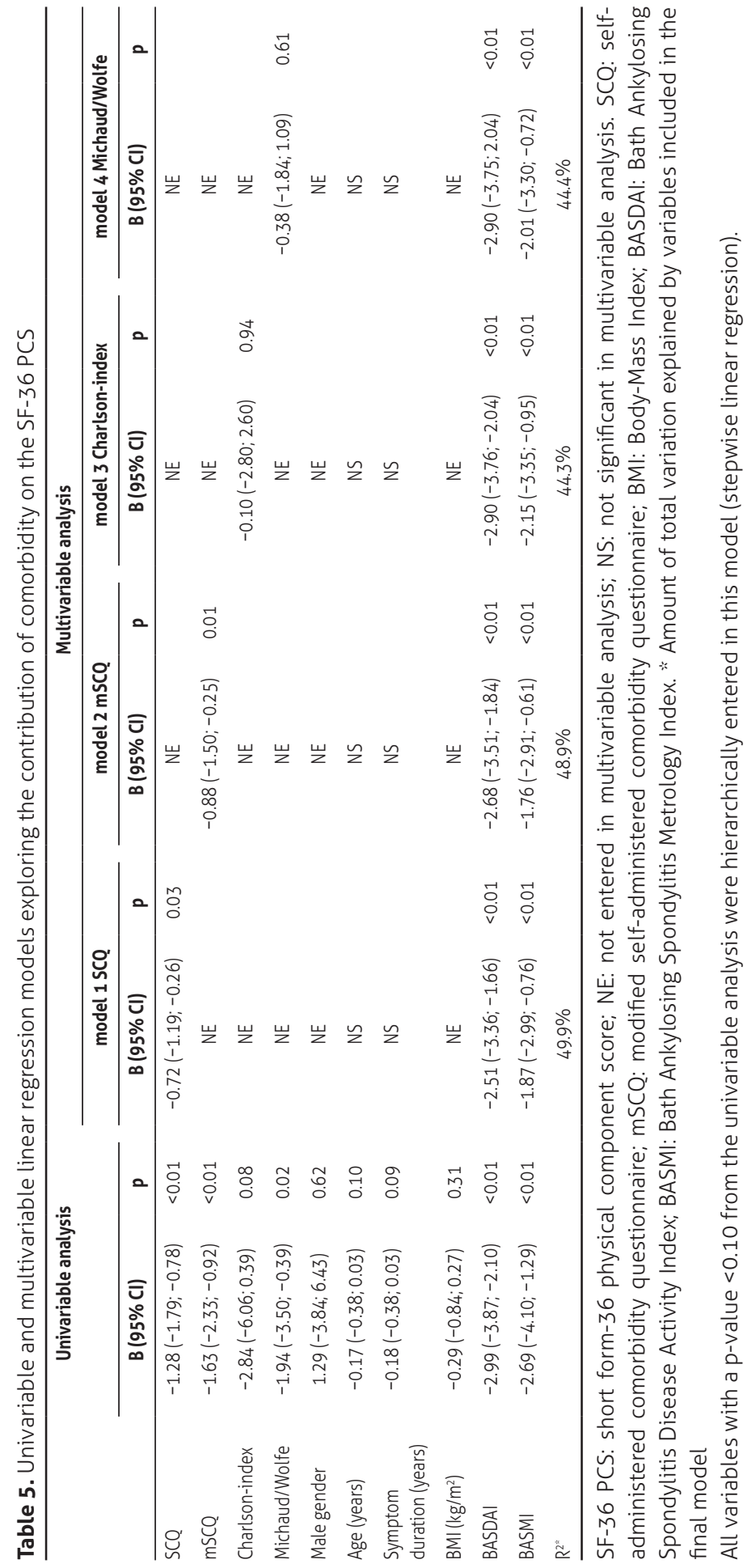




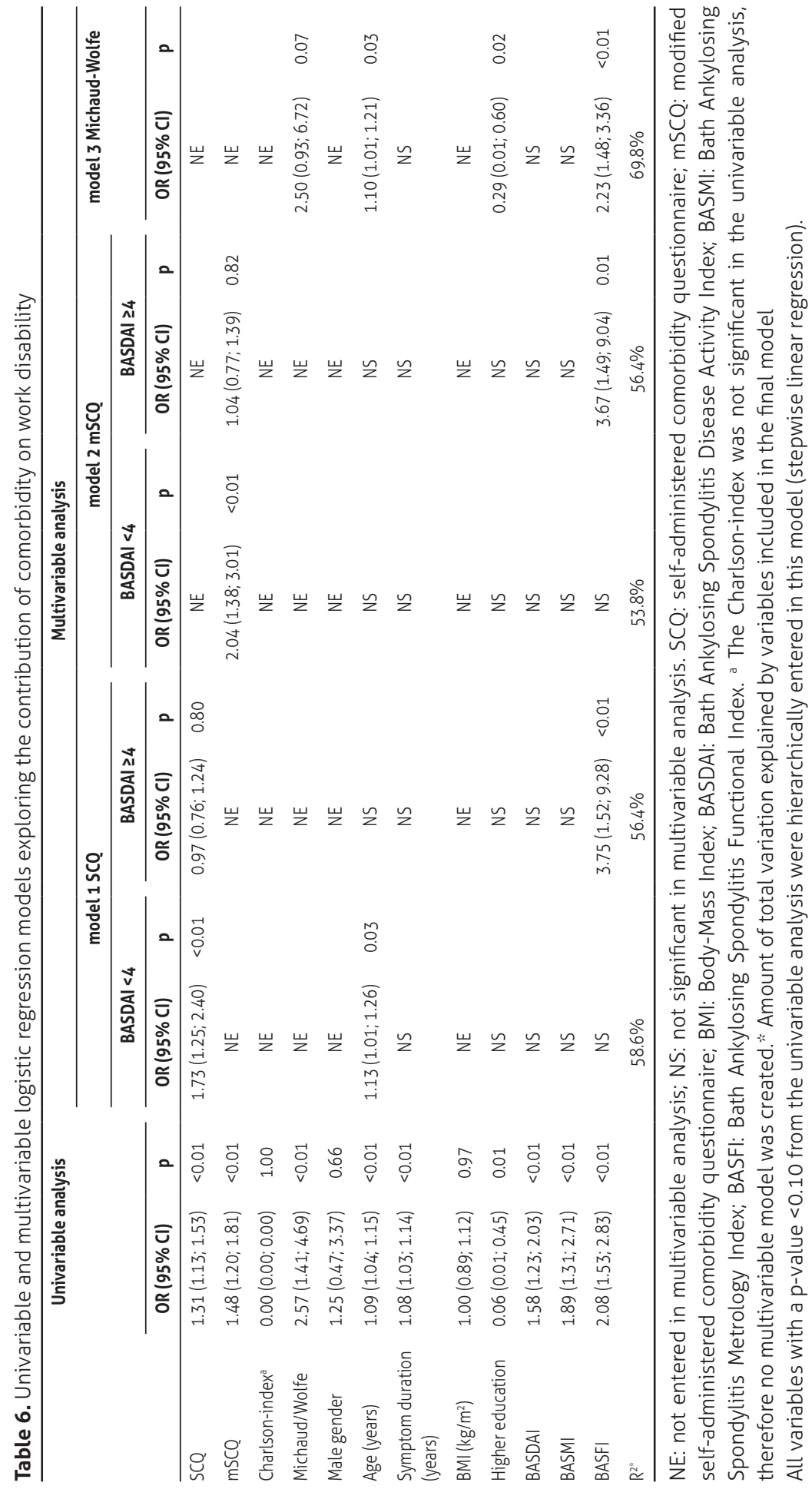


whereas the Charlson-index was not. A significant interaction between disease activity and both SCO and $\mathrm{mSCO}$ with work disability was found; therefore the models were stratified for level of disease activity in multivariable analysis. In patients with low disease activity (BASDAI <4, n=46), comorbidity (SCO or mSCO) was the only variable which was significantly associated with work disability. In patients with high disease activity (BASDAI $\geq 4, n=37$ ), work disability was not associated with comorbidity. The Michaud/ Wolfe index was not associated with work disability in multivariable analysis.

\section{DISCUSSION}

The present study shows that the SCQ has evidence for validity in an outpatient population with long-standing AS. Agreement between self-reported comorbidities and comorbidities retrieved from the medical records was moderate to almost perfect for the nonrheumatic conditions, except for ulcer and stomach disease and for depression, which were both more frequently reported by patients than retrieved from the medical records. Possibly, patients may have reported a disease based on symptoms they are experiencing without having an official diagnosis by a physician or patients may have been diagnosed by a general practitioner or, less likely, by a physician working outside the region of our hospitals. With respect to the rheumatic conditions, the agreement for osteoarthritis was low in both directions, which means that also some patients were unaware that they suffered from osteoarthritis, although it was stated in the medical record. Agreement for low back pain (reported by $68 \%$ of patients) and chronic rheumatic disease (reported by $15 \%$ of patients) was not calculable as by default patients with AS were considered to have these conditions. Partly, patients might have ignored the instruction to report only 'additional' diseases. For chronic rheumatic disease, the presence of peripheral arthritis was not of influence on this reporting. The low criterion validity for the rheumatic items supports our hypothesis that it is difficult for AS patients to distinguish the index disease and its impact on health outcomes from other rheumatic conditions, and supports the proposal to remove these items when applying the SCO in AS. Overall, the agreement observed in our study was largely comparable with that reported in the original validation study [17]. Other studies confirmed also that a self-reported instrument is a reliable data source for assessment of comorbidities [34, 35].

The SCO has two important characteristics. First, it is a self-report questionnaire, which offers a feasible, but possibly less accurate, method to collect comorbidity data. Second, the SCO includes functional limitations due to the comorbidity, which may better predict HRQoL and physical function than medical records data. These two characteristics make the SCQ a promising instrument to adjust commonly used outcomes in AS for the impact of comorbidities. The correlation of the original SCQ with the Michaud/Wolfe index was 
stronger than with the Charlson index, which is as expected and consistent with the conceptual difference between instruments: while the Michaud/Wolfe index was developed to understand the influence of comorbidity on several health outcomes, the Charlson index was developed to predict mortality and therefore does not necessarily include diseases with high impact on physical function and HROoL (such as rheumatoid arthritis or depression). Both correlations improved when excluding the rheumatic items from the SCO. Nevertheless, the correlations between the comorbidity indices were not perfect, which is most likely due to the different conditions included in the different indices and to the additional points, which can be scored in the SCO based on self-perceived impact on function. The correlation between the mSCO and Charlson index was similar to the correlation between the SCO and Charlson index found in the original study of the SCO $(r=0.32$ versus $r=0.36$ in our study) [17].

Our a priori hypotheses on the correlations with other constructs were largely met and confirmed correlation of both the SCO and mSCO with physical function, HROoL, and work disability. The SCQ but not the $\mathrm{MSCQ}$ showed a weak correlation with measures of AS-related disease activity. This supports a better validity for measuring comorbidity with the $\mathrm{mSCO}$. It is well recognized that the experienced functional limitations due to AS are partly influenced by disease activity [36], explaining the weak correlation between the SCO and AS-related disease activity when keeping the rheumatic items and its impact on function into the questionnaire. The adequate construct validity of the $\mathrm{mSCO}$ found in de present study is consistent with a previous study evaluating an adapted version of the SCO in patients with early inflammatory arthritis [20]. A study in patients with SLE and systemic sclerosis, however, showed that these patients could not distinguish comorbidities from their index disease. The results from these studies confirm the importance of removing comorbidities, which have overlapping symptoms with the index disease, from the questionnaire [21]. Importantly, also when adjusting for demographic and disease characteristics, the SCQ and mSCQ had a significant contribution to HROoL, physical function and work disability, while this was neither the case for the Charlson index nor for the Michaud/Wolfe index. Again, the additional questions on functional limitations for each condition in the SCO may explain the stronger association with physical function and HROoL. Interestingly, the SCO and mSCO correlated with work disability in patients with lower disease activity, while this was not the case in patients with high disease activity. Apparently, in these patients comorbidity and not the impact of AS on function was the main reason for withdrawal from work.

Another interesting observation was that a small a proportion of patients with AS reported spontaneously extra-articular manifestations (uveitis, psoriasis, IBD) in the open item section of the SCO. According to the SpA concept, these manifestations are part of the disease and should therefore not be considered as comorbidities, but this might have been confusing for patients. However, these concept-related manifestations may also 
influence HRQoL, physical function and work disability, in particular because they have a high prevalence in patients with AS [37-39]. An AS-specific questionnaire including both comorbidities and SpA concept-related manifestations might therefore be preferred above a generic questionnaire on comorbidities only, but future studies are needed to investigate this.

Some limitations of our study need to be addressed. First, most comorbid conditions were not prevalent and the large 95\% confidence intervals of the kappa statistics found in the present study may have been due to the low prevalence. Second, because the SCQ was administered in year 8 of follow-up of the OASIS-study, 64 out of 162 Dutch and Belgian patients who were included at baseline, were lost to follow up. It is unclear if this was also partly a result of comorbidity. However, we expect that the loss to follow up will not have a significant influence on the validity results. Third, our population with long-standing AS may not be representative for all patients with AS. Therefore, the results should be confirmed in patients with early AS. Fourth, the Michaud/Wolfe index was not yet developed at the time the patients in the present study completed the SCO. Therefore we could only calculate this score retrospectively based on medical records data, although it is officially a self-report questionnaire. This may have influenced the scores and hampers a formal comparison of the SCQ and Michaud/Wolfe index. Fifth, we compared the SCQ items with medical record retrieved comorbidities. Medical records may have their limitations and quality of documentation can vary. We only used medical records available in the hospitals in which the patients were treated for AS and we did not include documentation from external sources unless clearly stated in the medical records of the patient. This may have underestimated the accuracy of self-report. Sixth, neither pilot testing of the questionnaire prior to its use nor test-retest reliability of the SCQ was done. For the original English study, pilot-testing was performed and a good test-retest reliability of the SCO was reported. We did not expect differences in the translated Dutch version for AS, since the questionnaire does not contain words that we expect to be ambiguous and the medical terms are those that are also commonly used by patients and lay persons in our countries. Lastly, the study sample was too small to test the predictive validity with relation to future work disability, functional decline or resource utilization. This aspect should be part of the future research agenda on measurement of comorbidities in AS.

In summary, our study showed that patients with AS can accurately report most comorbidities included in the SCO and confirms that the SCO correlates well with physical function and HROOL in patients with AS. The aspects of truth of the SCO improved after removing rheumatic items from the questionnaire in the $\mathrm{mSCO}$. It should be realized that SpA concept-related manifestations (uveitis, IBD, psoriasis), are not included in this index, although they may have an important additional impact on health status. 


\section{REFERENCES}

1. Ariza-Ariza R, Hernandez-Cruz B, Navarro-Sarabia F. Physical function and health-related quality of life of Spanish patients with ankylosing spondylitis. Arthritis Rheum 2003:49:483-7.

2. Salaffi F, Carotti M, Gasparini S, et al. The health-related quality of life in rheumatoid arthritis, ankylosing spondylitis, and psoriatic arthritis: a comparison with a selected sample of healthy people. Health Qual Life Outcomes 2009;7:25.

3. Boonen A. A review of work-participation, cost-of-illness and cost-effectiveness studies in ankylosing spondylitis. Nat Clin Pract Rheumatol 2006;2:546-53.

4. Braun J, Sieper J. Ankylosing spondylitis. Lancet 2007;369:1379-90.

5. Mathieu S, Gossec L, Dougados M, et al. Cardiovascular profile in ankylosing spondylitis: a systematic review and meta-analysis. Arthritis Care Res (Hoboken) 2011;63:557-63.

6. Peters MJ, Visman I, Nielen MM, et al. Ankylosing spondylitis: a risk factor for myocardial infarction? Ann Rheum Dis 2010;69:579-81.

7. Kang JH, Chen YH, Lin HC. Comorbidity profiles among patients with ankylosing spondylitis: a nationwide population-based study. Ann Rheum Dis 2010;69:1165-8.

8. Bremander A, Petersson IF, Bergman S, et al. Population-based estimates of common comorbidities and cardiovascular disease in ankylosing spondylitis. Arthritis Care Res (Hoboken) 2011;63:550-6.

9. de Groot V, Beckerman H, Lankhorst G], et al. How to measure comorbidity. a critical review of available methods. J Clin Epidemiol 2003;56:221-9.

10. Fortin M, Dubois MF, Hudon C, et al. Multimorbidity and quality of life: a closer look. Health Qual Life Outcomes 2007:5:52.

11. Greenfield S, Apolone G, McNeil B], et al. The importance of co-existent disease in the occurrence of postoperative complications and one-year recovery in patients undergoing total hip replacement. Comorbidity and outcomes after hip replacement. Med Care 1993;31:141-54.

12. Greenfield S, Aronow HU, Elashoff RM, et al. Flaws in mortality data. The hazards of ignoring comorbid disease. JAMA 1988;260:2253-5.

13. Katz JN, Lipson S], Larson MG, et al. The outcome of decompressive laminectomy for degenerative lumbar stenosis. J Bone Joint Surg Am 1991;73:809-16.

14. Katz JN, Wright EA, Guadagnoli E, et al. Differences between men and women undergoing major orthopedic surgery for degenerative arthritis. Arthritis Rheum 1994;37:687-94.

15. Charlson ME, Pompei P, Ales KL, et al. A new method of classifying prognostic comorbidity in longitudinal studies: development and validation. J Chronic Dis 1987;40:373-83.

16. Michaud K, Wolfe F. The development of a rheumatic disease research comorbidity index for use in outpatients with RA, OA, SLE and fibromyalgia (FMS) [abstract]. Arthritis Rheum 2007:Suppl 56:S596.

17. Sangha O, Stucki G, Liang MH, et al. The Self-Administered Comorbidity Questionnaire: a new method to assess comorbidity for clinical and health services research. Arthritis Rheum 2003;49:156-63.

18. Ariza-Ariza R, Hernandez-Cruz B, Lopez-Antequera G, et al. Variables related to utility in patients with ankylosing spondylitis. Clin Rheumatol 2009:28:207-11.

19. Boonen A, Chorus A, Miedema H, et al. Withdrawal from labour force due to work disability in patients with ankylosing spondylitis. Ann Rheum Dis 2001;60:1033-9.

20. Baron M, Schieir O, Hudson M, et al. Evaluation of the clinimetric properties of the Early Inflammatory Arthritis--self-administered comorbidity questionnaire. Rheumatology 2009;48:390-4.

21. Hudson M, Bernatsky S, Taillefer S, et al. Patients with systemic autoimmune diseases could not distinguish comorbidities from their index disease. Journal of clinical epidemiology 2008;61:654-62.

22. Spoorenberg A, van der Heijde D, de Klerk E, et al. Relative value of erythrocyte sedimentation rate and Creactive protein in assessment of disease activity in ankylosing spondylitis. The Journal of rheumatology 1999;26:980-4.

23. van der Linden S, Valkenburg HA, Cats A. Evaluation of diagnostic criteria for ankylosing spondylitis. A proposal for modification of the New York criteria. Arthritis Rheum 1984;27:361-8. 
24. Mokkink LB, Terwee CB, Patrick DL, et al. The COSMIN checklist for assessing the methodological quality of studies on measurement properties of health status measurement instruments: an international Delphi study. Quality of life research: an international journal of quality of life aspects of treatment, care and rehabilitation 2010;19:539-49.

25. Wolfe F, Michaud K, Li T, et al. Chronic conditions and health problems in rheumatic diseases: comparisons with rheumatoid arthritis, noninflammatory rheumatic disorders, systemic lupus erythematosus, and fibromyalgia. J Rheumatol 2010;37:305-15.

26. Calin A, Garrett S, Whitelock H, et al. A new approach to defining functional ability in ankylosing spondylitis: the development of the Bath Ankylosing Spondylitis Functional Index. ] Rheumatol 1994;21:2281-5.

27. VanderZee KI, Sanderman R, Heyink JW, et al. Psychometric qualities of the RAND 36-Item Health Survey 1.0: a multidimensional measure of general health status. Int ] Behav Med 1996;3:104-22.

28. Aaronson NK, Muller M, Cohen PD, et al. Translation, validation, and norming of the Dutch language version of the SF-36 Health Survey in community and chronic disease populations. Journal of clinical epidemiology 1998;51:1055-68.

29. Ware JE, Jr., Sherbourne CD. The MOS 36-item short-form health survey (SF-36). I. Conceptual framework and item selection. Med Care 1992;30:473-83.

30. Doward LC, Spoorenberg A, Cook SA, et al. Development of the ASOoL: a quality of life instrument specific to ankylosing spondylitis. Ann Rheum Dis 2003;62:20-6.

31. Garrett S, Jenkinson T, Kennedy LG, et al. A new approach to defining disease status in ankylosing spondylitis: the Bath Ankylosing Spondylitis Disease Activity Index. J Rheumatol 1994;21:2286-91.

32. van der Heijde D, Lie E, Kvien TK, et al. ASDAS, a highly discriminatory ASAS-endorsed disease activity score in patients with ankylosing spondylitis. Ann Rheum Dis 2009;68:1811-8.

33. Landis JR, Koch GG. The measurement of observer agreement for categorical data. Biometrics 1977;33: 159-74.

34. Katz JN, Chang LC, Sangha O, et al. Can comorbidity be measured by questionnaire rather than medical record review? Med Care 1996;34:73-84.

35. Kriegsman DM, Penninx BW, van Eijk JT, et al. Self-reports and general practitioner information on the presence of chronic diseases in community dwelling elderly. A study on the accuracy of patients' self-reports and on determinants of inaccuracy. J Clin Epidemiol 1996;49:1407-17.

36. Machado P, Landewe R, Braun J, et al. A stratified model for health outcomes in ankylosing spondylitis. Ann Rheum Dis 2011;70:1758-64.

37. Chorus AM, Miedema HS, Boonen A, et al. Quality of life and work in patients with rheumatoid arthritis and ankylosing spondylitis of working age. Ann Rheum Dis 2003;62:1178-84.

38. Brophy S, Pavy S, Lewis P, et al. Inflammatory eye, skin, and bowel disease in spondyloarthritis: genetic, phenotypic, and environmental factors. ] Rheumatol 2001;28:2667-73.

39. Robertson LP, Davis MJ. A longitudinal study of disease activity and functional status in a hospital cohort of patients with ankylosing spondylitis. Rheumatology (Oxford) 2004:43:1565-8. 


\section{CHAPTER 7 \\ Ankylosing Spondylitis and Risk of Ischemic Heart Disease: a Population-Based Cohort Study}

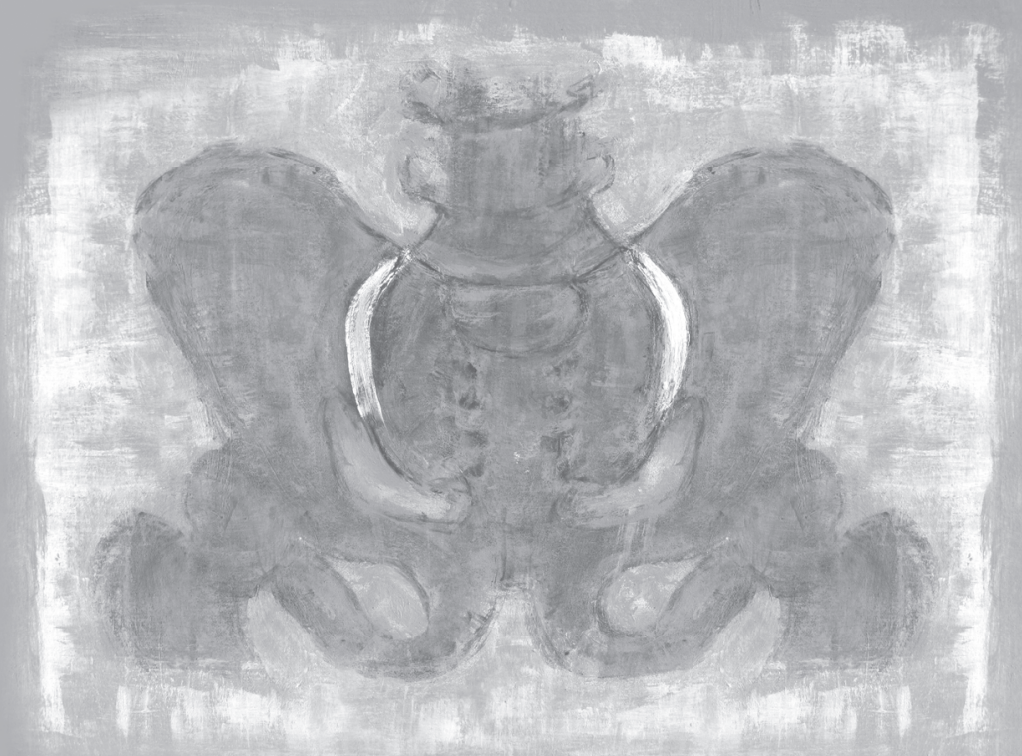

Ivette Essers, Carmen Stolwijk, Annelies Boonen, Marie L. De Bruin, Marloes T. Bazelier, Frank de Vries, Astrid van Tubergen 


\section{ABSTRACT}

\section{Objective}

To investigate the incidence and risk of ischemic heart disease (IHD) and acute myocardial infarction (AMI), including the role of non-steroidal-anti-inflammatory drugs (NSAIDs), in patients with ankylosing spondylitis (AS) compared with population controls.

\section{Methods}

All patients with newly diagnosed AS $(n=3,809)$ from the British Clinical Practice Research Datalink (1987-2012) were matched with up to 7 persons without AS by year of birth, gender and practice $(n=26,197)$. Incidence rate ratios (IRRs) and hazard ratios (HR) for development of IHD and AMI were calculated. Stepwise analyses were performed adjusting for age, gender, comorbidity, and drug use, including NSAIDs.

\section{Results}

At baseline, $4.3 \%$ of the patients had IHD and $1.8 \%$ had $\mathrm{AMI}$, compared with $3.4 \%$ and $1.4 \%$ of the controls, respectively. After exclusion of pre-existing IHD or AMI, the IRRs were 1.18 (95\%-confidence interval [Cl] 0.96-1.46) and 0.91 (95\%-Cl 0.65-1.27) for IHD and $\mathrm{AMI}$, respectively. Compared with controls, the age-gender adjusted HR for developing IHD was 1.20 (95\%-Cl 0.97-1.48), and for AMI 0.91 (95\%-Cl 0.65-1.28). In female patients, the risk of developing IHD was increased (HR 1.88, 95\%-Cl 1.22-2.90), but after adjustment for all possible risk factors only a non-significant trend was found (HR 1.31, 95\%-CI 0.83-2.08). In particular, NSAID use explained this change (HR IHD adjusted for age-gender-NSAID use 1.57, 95\%-Cl 0.99-2.48).

\section{Conclusion}

Female patients with AS had an increased age adjusted risk of developing IHD, but after adjustment for NSAID use only a non-significant trend towards increased risk was found. 


\section{INTRODUCTION}

Ischemic heart disease (IHD) is one of the leading causes of death and loss of quality of life worldwide [1]. In the last decades, an excess in cardiovascular (CV) morbidity and mortality in patients with chronic inflammatory rheumatic disease, such as rheumatoid arthritis (RA) has been demonstrated $[2,3]$. This led to additional recommendations for CV risk assessment [4], and treatment guidelines to manage the CV risk in RA [5]. In ankylosing spondylitis (AS) conflicting results on the risk of CV disease have been reported. [6] An increased risk of IHD in AS was found in three studies [7-9]. Also a statistically significantly increased risk for acute myocardial infarction (AMI) in AS was reported in some studies [10-12], whereas others failed to demonstrate this [7, 13, 14]. Differences in selection of populations or a flawed study design may have contributed to the contradictory results [15].

Several etiological mechanisms could be associated with the increased CV risk in AS. First, the systemic inflammation, which is a part of the pathophysiology of AS, may play an independent role in the onset of atherosclerosis [6]. Second, several studies have shown an increased prevalence of conventional risk factors in AS, including the metabolic syndrome and decreased levels of HDL cholesterol $[6,11]$. Third, the long term use of non-steroidal anti-inflammatory drugs (NSAIDs) may accelerate the atherosclerotic process $[6,16]$. NSAIDs are the cornerstone of the treatment of AS, and are often prescribed on a long-term basis. According to a recent evaluation of all available evidence by the European Medicines Agency, NSAIDs as a class are associated with a small increased risk of atherosclerosis or atherothrombotic events, particularly in patients with underlying heart or circulatory conditions, or with certain CV risk factors [17]. Moreover, high dose or long-term NSAID use may increase this risk [18]. Differences among the several types of NSAIDs with respect to the CV risk have been reported $[17,19]$.

To date, there are still some unsolved epidemiologic issues on the CV risk of patients with AS. Studies comparing the CV risk in patients with AS with population-based controls are limited or had a flawed study design. Also, studies including adjustments for other potential risk factors besides age and gender when analyzing the risk of CV disease in AS, are scarce. Furthermore, the role of NSAID use in the etiology of AS associated IHD has to our knowledge never been assessed. Therefore, the objective of the present study was to investigate the risk of a first event of IHD, including AMI, in patients with AS compared with population-based controls, and the role of NSAID use in this. 


\section{METHODS}

\section{Design and data source}

A retrospective cohort study was conducted using data from the Clinical Practice Research Datalink GOLD (CPRD), formerly known as the General Practice Research Database. CPRD contains computerized medical records of over 10 million patients under care of general practitioners (GPs) in the United Kingdom. Since 1987, data are prospectively recorded, and include patient demographics, prescription details, clinical events, preventive care provided, specialist referrals, hospital admissions and their major outcomes. Read codes classify diseases and symptoms. Practices only contribute to CPRD when their data quality is up to research standards. CPRD has been extensively validated [20].

\section{Study population}

The study population consisted of all patients aged 16 years or older with at least one recording of AS during the period of CPRD data collection, which started for the present study in January 1987 and ended in December 2012. The start of valid data collection of each patient was defined as the date at which the practice was included into CPRD, the GP's data set was approved as 'up-to-standard', and the patient moved into the practice. Patients with a first-ever diagnosis of AS were matched by year of birth, gender, calendar time, and practice to up to 7 control subjects without a diagnosis of AS at any time during their registration period. The date of the first record of AS defined the index date. Control patients were assigned the same index date as their matched case. The subjects in the study population were followed from their index date to the outcome of interest, the end of data collection, the date of transfer out of the practice area or death, whichever came first. Patients and controls with a record for another inflammatory rheumatic disease (RA, psoriatic arthritis, systemic lupus erythematous or vasculitis) any time during the enrolment were excluded from the current analysis.

\section{Study outcome and risk factors}

Outcomes of interest were a first event of IHD or AMI, specified by read codes. IHD was defined as all types of ischemic heart disease, and included for example AMI, coronary artery bypass surgery and percutaneous coronary intervention. The total follow-up time was divided into 30-day intervals, in order to adjust the analyses for the influence of potential confounders in a time-varying way. The computerized medical records before the start of each interval were reviewed for the occurrence of potential confounders. Baseline confounders considered included gender, body mass index (BMI), smoking status, and alcohol use (the later three as dummy variables). The following time-varying confounders were considered: age, hypercholesterolemia (including familial hypercholesterolemia), a history of acute or chronic renal failure, as well as a prescription of antihypertensives, 
antidiabetics, antiplatelet agents, statins, and asthma medication six months before the start of an interval. Exposure to NSAIDs was determined as the average defined daily doses (DDD) equivalent to $100 \mathrm{mg}$ of diclofenac, based on the World Health Organization prior to the start of an interval.[21] NSAIDs were further categorized into cyclooxygenase-2 (COX-2) inhibitors, naproxen, or other traditional NSAIDs 3 months prior the start of an interval (binary), because of possible different impact on the risk of IHD or AMI [17].

\section{Statistical analysis}

Dichotomous baseline characteristics (excluding gender and age) of patients and controls were compared using chi-square tests. Stratified analyses for gender were done with respect to a history of IHD and AMI before the index date. After excluding patients with pre-existing IHD or AMI, incidence rates (IR) for IHD and AMI were calculated for patients (and their controls), respectively, and were estimated as the number of subjects with the event per 1,000 person-years. Person-years (pys) were computed by adding all person-time from the index date to either the date of the first event or to the date of censoring if the event did not occur. Incidence rate ratios (IRRs) were calculated by dividing the IR for patients by the IR for controls. IRRs were stratified based on gender and age categories (16-39, 40-49, 50-59, 60-69, 70-79 and $\geq 80$ years). The overall prevalence of IHD and AMI was estimated using Kaplan-Meier methods and included also patients (and controls, respectively) with an event at baseline. Furthermore, the attributable risk per 1,000 pys was calculated for IHD and AMI. The risk of developing a first IHD or AMI after the index date was estimated by Hazard Ratios (HR) using time-dependent Cox proportional hazard models for those patients (and controls) without the outcome of interest before the index date. Three step-wise models were created with adjustment for potential confounders: 1) adjustment for age and gender, 2) adjustment for age, gender and the DDD of NSAID use, 3) adjustment for age, gender, DDD of NSAID use, and all confounders that changed the beta coefficient of the HR more than $1 \%$ in the age-gender adjusted analysis. Stratified analyses were done for gender.

In a separate analysis, the role of NSAIDs in the risk of IHD was evaluated. For this, all patients were stratified according to the recent use of any NSAIDs, naproxen, COX-2 inhibitors, or other traditional NSAIDs. HRs were calculated, in which patients were compared with controls, irrespective of their NSAID use. In addition, patients with a history of NSAID use were compared with patients without a history of the same class of NSAIDs, using the Wald test. This analysis was also stratified for gender. All statistical analyses were conducted using SAS 9.1. 


\section{RESULTS}

\section{Baseline results}

Baseline characteristics of patients with AS $(n=3,809)$ and their matched controls $(n=26,197,92 \%$ had 7 controls) are presented in Table 1 . The mean duration of followup for patients and controls was 6.6 years. At baseline, patients were more likely to have been diagnosed with IHD (4.3\% vs $3.4 \%$ ) or AMI (1.8\% vs $1.4 \%)$ compared with controls (Table 1). In the stratified analyses, male patients were more likely to have been diagnosed with IHD ( $3.4 \%$ vs $2.8 \%, p=0.04)$ and AMI ( $1.7 \%$ vs $1.2 \%, p=0.01)$ compared with male controls, and female patients were more likely to have been diagnosed with IHD (0.9\% vs $0.6 \%, p=0.04)$, but not with $\mathrm{AMI}(0.1 \%$ vs $0.1 \%, \mathrm{p}=0.06)$ compared with female controls. Within the AS population, male patients had more often a history of IHD $(p=0.01)$ and AMI $(p<0.01)$ compared with female patients.

Table 1. Baseline characteristics of patients with ankylosing spondylitis and controls

\begin{tabular}{|c|c|c|c|c|c|c|}
\hline \multicolumn{2}{|l|}{ Characteristic } & \multicolumn{2}{|c|}{ Patients with AS } & \multicolumn{2}{|c|}{ Controls } & \multirow[t]{2}{*}{ P-value } \\
\hline & & $N=3,809$ & $(\%)$ & $N=26,197$ & $(\%)$ & \\
\hline \multicolumn{2}{|l|}{ Males } & 2,686 & $(70.5 \%)$ & 18,519 & $(70.7 \%)$ & - \\
\hline \multirow[t]{7}{*}{ Age at index date } & $16-29$ & 728 & $(19.1 \%)$ & 5,085 & $(19.4 \%)$ & - \\
\hline & $30-39$ & 1,038 & $(27.3 \%)$ & 7,172 & $(27.4 \%)$ & - \\
\hline & $40-49$ & 817 & $(21.4 \%)$ & 5,674 & $(21.7 \%)$ & - \\
\hline & $50-59$ & 570 & $(15.0 \%)$ & 3,870 & $(14.8 \%)$ & - \\
\hline & $60-69$ & 376 & (9.9\%) & 2,551 & $(9.7 \%)$ & - \\
\hline & $70-79$ & 206 & $(5.4 \%)$ & 1,399 & $(5.3 \%)$ & - \\
\hline & $80+$ & 74 & $(1.9 \%)$ & 446 & $(1.7 \%)$ & - \\
\hline \multirow[t]{5}{*}{ BMI } & $<20$ & 204 & $(5.4 \%)$ & 1,280 & $(4.9 \%)$ & 0.21 \\
\hline & $20-25$ & 1,046 & $(27.5 \%)$ & 6,934 & $(26.5 \%)$ & 0.20 \\
\hline & $25-30$ & 879 & $(23.1 \%)$ & 6,066 & $(23.2 \%)$ & 0.91 \\
\hline & $>30$ & 447 & $(11.7 \%)$ & 3,086 & $(11.8 \%)$ & 0.94 \\
\hline & Unknown & 1,223 & $(32.4 \%)$ & 8,831 & $(33.7 \%)$ & 0.10 \\
\hline \multirow[t]{4}{*}{ Smoking status } & Never & 1,668 & $(43.8 \%)$ & 11,962 & $(45.7 \%)$ & 0.03 \\
\hline & Current & 1,403 & $(36.8 \%)$ & 8,256 & $(31.5 \%)$ & $<0.01$ \\
\hline & Ex & 569 & $(14.9 \%)$ & 3,605 & $(13.8 \%)$ & 0.05 \\
\hline & Unknown & 169 & $(4.4 \%)$ & 2,374 & $(9.1 \%)$ & $<0.01$ \\
\hline \multirow[t]{3}{*}{ Alcohol use } & Yes & 2,612 & $(68.6 \%)$ & 17,468 & $(66.7 \%)$ & 0.02 \\
\hline & No & 596 & $(15.6 \%)$ & 3,251 & $(12.4 \%)$ & $<0.01$ \\
\hline & Unknown & 601 & $(15.8 \%)$ & 5,478 & $(20.9 \%)$ & $<0.01$ \\
\hline \multicolumn{7}{|l|}{ Disease history } \\
\hline \multicolumn{2}{|c|}{ Any cardiovascular disease } & 216 & $(5.7 \%)$ & 1,317 & $(5.0 \%)$ & 0.09 \\
\hline
\end{tabular}


Table 1 (continued)

\begin{tabular}{|c|c|c|c|c|c|c|}
\hline \multirow[t]{2}{*}{ Characteristic } & & \multicolumn{2}{|c|}{ Patients with AS } & \multicolumn{2}{|c|}{ Controls } & \multirow[t]{2}{*}{ P-value } \\
\hline & & $N=3,809$ & (\%) & $N=26,197$ & $(\%)$ & \\
\hline & IHD & 164 & $(4.3 \%)$ & 898 & $(3.4 \%)$ & $<0.01$ \\
\hline & AMI & 69 & $(1.8 \%)$ & 354 & $(1.4 \%)$ & 0.02 \\
\hline & Stroke & 44 & $(1.2 \%)$ & 317 & $(1.2 \%)$ & 0.77 \\
\hline & Heart failure & 24 & $(0.6 \%)$ & 108 & $(0.4 \%)$ & 0.06 \\
\hline & $\begin{array}{l}\text { Peripheral vascular } \\
\text { disease }\end{array}$ & 38 & $(1.0 \%)$ & 246 & $(0.9 \%)$ & 0.73 \\
\hline Hypertension & & 316 & $(8.3 \%)$ & 2,007 & $(7.7 \%)$ & 0.17 \\
\hline Acute renal failure & & 3 & $(0.1 \%)$ & 16 & $(0.1 \%)$ & 0.69 \\
\hline Chronic renal failure & & 2 & $(0.1 \%)$ & 62 & $(0.2 \%)$ & 0.02 \\
\hline Hypercholesterolemia & & 165 & $(4.3 \%)$ & 1,127 & $(4.3 \%)$ & 0.93 \\
\hline Diabetes Mellitus & & 150 & $(3.9 \%)$ & 795 & $(3.0 \%)$ & $<0.01$ \\
\hline \multicolumn{7}{|c|}{ History of drug use 6 months before the index date } \\
\hline \multirow[t]{6}{*}{ Any antihypertensives } & & 509 & $(13.4 \%)$ & 2,902 & $(11.1 \%)$ & $<0.01$ \\
\hline & Beta-blockers & 199 & $(5.2 \%)$ & 1,276 & $(4.9 \%)$ & 0.35 \\
\hline & Loop diuretics & 92 & $(2.4 \%)$ & 380 & $(1.5 \%)$ & $<0.01$ \\
\hline & Thiazide diuretics & 173 & $(4.5 \%)$ & 967 & $(3.7 \%)$ & 0.01 \\
\hline & ACE-I or ANG-II-R & 248 & $(6.5 \%)$ & 1,449 & $(5.5 \%)$ & 0.01 \\
\hline & $\begin{array}{l}\text { Calcium channel } \\
\text { antagonist }\end{array}$ & 177 & $(4.7 \%)$ & 985 & $(3.8 \%)$ & $<0.01$ \\
\hline Antiplatelet agents & & 206 & $(5.4 \%)$ & 1,142 & $(4.4 \%)$ & $<0.01$ \\
\hline Nitrates & & 94 & $(2.5 \%)$ & 391 & $(1.5 \%)$ & $<0.01$ \\
\hline Antidiabetics \# & & 107 & $(2.8 \%)$ & 586 & $(2.2 \%)$ & 0.03 \\
\hline Statins & & 233 & $(6.1 \%)$ & 1,363 & $(5.2 \%)$ & 0.02 \\
\hline Asthma medication $\$$ & & 289 & $(7.6 \%)$ & 1,661 & $(6.3 \%)$ & $<0.01$ \\
\hline \multirow[t]{4}{*}{ NSAIDs } & & 1,731 & $(45.4 \%)$ & 2,163 & $(8.3 \%)$ & $<0.01$ \\
\hline & COX-2 inhibitors & 225 & $(5.9 \%)$ & 114 & $(0.4 \%)$ & $<0.01$ \\
\hline & Naproxen only & 303 & $(8.0 \%)$ & 298 & $(1.1 \%)$ & $<0.01$ \\
\hline & $\begin{array}{l}\text { Other traditional } \\
\text { NSAIDs }\end{array}$ & 1,582 & $(41.5 \%)$ & 2,071 & $(7.9 \%)$ & $<0.01$ \\
\hline
\end{tabular}

A chi-square test was performed to compare patients with AS and controls

\# Antidiabetics, including insulin

\$ Asthma medication, including bronchodilators, inhaled corticosteroids, leukotriene receptor antagonists, beta-2 agonists, and theophylline,

AS, Ankylosing spondylitis; BMI, body mass index; IHD, ischemic heart disease; AMI, acute myocardial infarction; ACE-I, angiotensin converting enzyme inhibitor; ANG-II-R, angiotensin II receptor antagonist; COX-2 inhibitors, Cyclooxygenase-2 inhibitor; NSAIDs, non-steroidal anti-inflammatory drugs 


\section{Incidence and overall prevalence of ischemic heart disease and acute myocardial infarction}

In Table 2, a trend towards a higher incidence of developing a first IHD event in patients compared with controls (IRR $1.18,95 \%-\mathrm{Cl} 0.96-1.46$ ) is shown. In particular, the IRR of IHD was increased among female patients versus female controls (IRR 1.72, 95\%-CI 1.12-2.64), whereas male patients were not at increased risk of IHD (IRR 1.07, 95\%-CI

0.84-1.37). The IRR of developing a first AMI event was not increased in patients versus controls (IRR 0.91, 95\%-Cl 0.65-1.27). Figure 1 visualizes the overall prevalence of

Table 2. Incidence rates of ischemic heart disease and acute myocardial infarction in patients with AS and controls

\begin{tabular}{|c|c|c|c|c|c|c|c|c|}
\hline & \multicolumn{3}{|c|}{ AS patients } & \multicolumn{3}{|c|}{ Controls } & \multirow{2}{*}{$\begin{array}{l}\text { Incidence } \\
\text { rate ratio }\end{array}$} & \multirow[t]{2}{*}{$95 \%-\mathrm{Cl}$} \\
\hline & Event & $\begin{array}{c}\text { Person- } \\
\text { years }\end{array}$ & $\begin{array}{c}\text { Incidence } \\
\text { rate }\end{array}$ & Event & $\begin{array}{c}\text { Person- } \\
\text { years }\end{array}$ & $\begin{array}{c}\text { Incidence } \\
\text { rate }\end{array}$ & & \\
\hline Ischemic heart disease ^ & 102 & 23,719 & 4.30 & 600 & 165,176 & 3.63 & 1.18 & $(0.96-1.46)$ \\
\hline \multicolumn{9}{|l|}{ By gender } \\
\hline Male & 76 & 16,495 & 4.61 & 497 & 115,956 & 4.29 & 1.07 & $(0.84-1.37)$ \\
\hline Female & 26 & 7,224 & 3.60 & 103 & 49,219 & 2.09 & 1.72 & $(1.12-2.64)$ \\
\hline \multicolumn{9}{|l|}{ By age } \\
\hline $16-39$ & 3 & 7,674 & 0.39 & 17 & 52,690 & 0.32 & 1.21 & $(0.36-4.13)$ \\
\hline $40-49$ & 9 & 6,367 & 1.41 & 74 & 43,523 & 1.70 & 0.83 & $(0.42-1.66)$ \\
\hline $50-59$ & 27 & 4,779 & 5.65 & 135 & 33,636 & 4.01 & 1.41 & $(0.93-2.13)$ \\
\hline $60-69$ & 35 & 3,081 & 11.36 & 180 & 21,435 & 8.40 & 1.35 & $(0.94-1.94)$ \\
\hline $70-79$ & 20 & 1,321 & 15.14 & 128 & 10,112 & 12.66 & 1.20 & $(0.75-1.92)$ \\
\hline $80+$ & 8 & 495 & 16.16 & 66 & 3,763 & 17.54 & 0.92 & $(0.44-1.92)$ \\
\hline Acute myocardial infarction \# & 38 & 24,560 & 1.55 & 291 & 170,551 & 1.71 & 0.91 & $(0.65-1.27)$ \\
\hline \multicolumn{9}{|l|}{ By gender } \\
\hline Male & 31 & 17,053 & 1.82 & 248 & 120,175 & 2.06 & 0.88 & $(0.61-1.28)$ \\
\hline Female & 7 & 7,507 & 0.93 & 43 & 5,0375 & 0.85 & 1.09 & $(0.49-2.43)$ \\
\hline \multicolumn{9}{|l|}{ By age category } \\
\hline $16-39$ & 1 & 7,683 & 0.13 & 9 & 52,823 & 0.17 & 0.76 & $(0.10-6.03)$ \\
\hline $40-49$ & 4 & 6,388 & 0.63 & 35 & 43,887 & 0.80 & 0.79 & $(0.28-2.21)$ \\
\hline $50-59$ & 12 & 4,921 & 2.44 & 59 & 34,680 & 1.70 & 1.43 & $(0.77-2.67)$ \\
\hline $60-69$ & 9 & 3,346 & 2.69 & 76 & 23,144 & 3.28 & 0.82 & $(0.41-1.63)$ \\
\hline $70-79$ & 7 & 1,594 & 4.39 & 68 & 11,601 & 5.86 & 0.75 & $(0.34-1.63)$ \\
\hline $80+$ & 5 & 626 & 7.98 & 44 & 4,399 & 10.00 & 0.80 & $(0.32-2.01)$ \\
\hline
\end{tabular}

* Number of patients or controls with an event / 1.000 person-years; ** The incidence rate ratio is calculated as the incidence rate for patients divided by the incidence rate of controls.

^ Because patients with a history of ischemic heart disease before or at the index date were excluded, the total number of AS patients was 3,640 and the total number of controls was 25,299 \# Because patients with a history of acute myocardial infarction before or at the index date were excluded, the total number of AS patients was 3,738 and the total number of controls was 25,843 

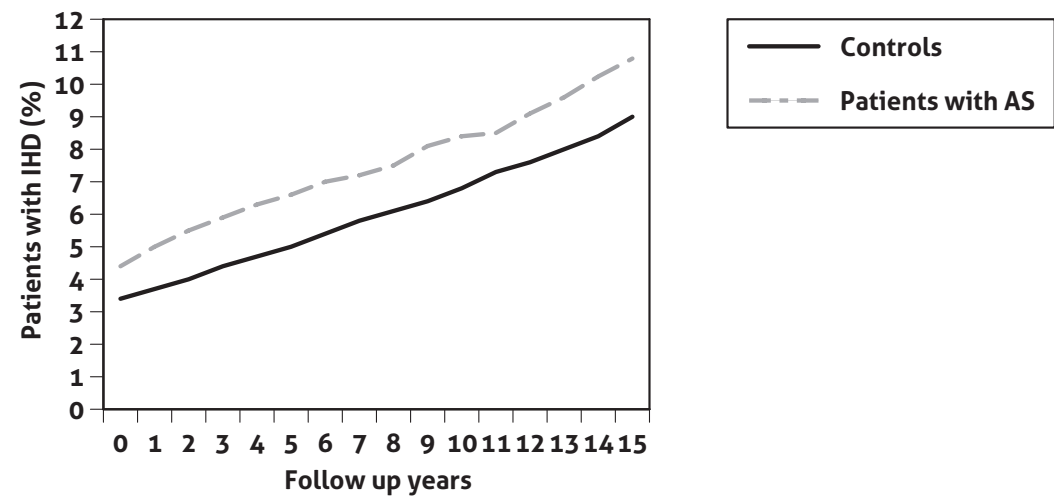

b Overall prevalence of acute myocardial infarction

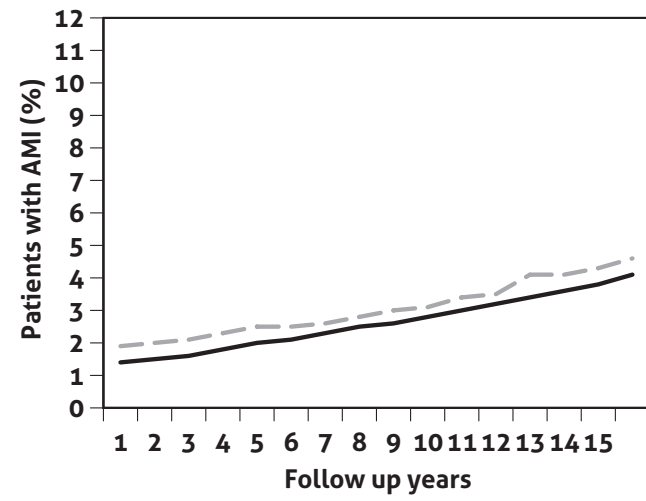

Figure 1 - Visualization of the overall prevalence over time, including baseline values, of ischemic heart disease (a) and acute myocardial infarction (b) in patients with AS and controls. For the calculation of the overall prevalence over time, also the patients (controls respectively) are included with an event at baseline. AMI: acute myocardial infarction, IHD: ischemic heart disease

IHD and AMI over time compared with controls, including events prior to baseline. At baseline, differences in the prevalences of IHD and AMI between patients and controls were found, which remained unchanged during follow-up. The overall prevalence of IHD was $10.8 \%$ in patients and $9.0 \%$ in controls, after 15 years of follow-up. The overall prevalence of AMI was $4.6 \%$ in patients and $4.1 \%$ in controls, after 15 years of follow-up. The attributable risk of AS for developing IHD was 0.7 per 1,000 pys, and for developing an AMI -0.2 per 1,000 pys.

\section{Risk of developing a first event of ischemic heart disease or acute myocardial infarction}

Table 3 shows that the risk of IHD or AMI was similar among patients with AS and controls. Female patients with AS had a 1.9-fold age-gender adjusted (adj) risk of IHD (HR 1.88, 
Table 3. Risk of ischemic heart disease and acute myocardial infarction in patients with AS compared with controls

\begin{tabular}{|c|c|c|c|c|c|c|c|}
\hline \multicolumn{8}{|c|}{ Ischemic heart disease ${ }^{\wedge}$} \\
\hline Population & $\begin{array}{c}\text { Number of } \\
\text { events }\end{array}$ & $\begin{array}{l}\text { Age-gender } \\
\text { adj HR }\end{array}$ & $95 \%-\mathrm{Cl}$ & NSAID adj HR ${ }^{1}$ & $95 \%-\mathrm{Cl}$ & Fully adj HR ${ }^{2}$ & $95 \%-\mathrm{Cl}$ \\
\hline Controls & 600 & Reference & & Reference & & Reference & \\
\hline AS patients & 102 & 1.20 & $(0.97-1.48)$ & 1.04 & $(0.83-1.30)$ & 1.00 & $(0.80-1.25)$ \\
\hline \multicolumn{8}{|l|}{ Gender } \\
\hline Female & 26 & 1.88 & $(1.22-2.90)$ & 1.57 & $(0.99-2.48)$ & 1.31 & $(0.83-2.08)$ \\
\hline Male & 76 & 1.07 & $(0.84-1.37)$ & 0.94 & $(0.72-1.21)$ & 0.94 & $(0.73-1.21)$ \\
\hline \multicolumn{8}{|c|}{ Acute Myocardial infarction \# } \\
\hline Population & $\begin{array}{c}\text { Number of } \\
\text { events }\end{array}$ & $\begin{array}{l}\text { Age-gender } \\
\text { adj HR }\end{array}$ & $95 \%-\mathrm{Cl}$ & NSAID adj HR ${ }^{1}$ & $95 \%-\mathrm{Cl}$ & Fully adj HR ${ }^{2}$ & $95 \%-\mathrm{Cl}$ \\
\hline Controls & 291 & Reference & & Reference & & Reference & \\
\hline AS patients & 38 & 0.91 & $(0.65-1.28)$ & 0.80 & $(0.56-1.15)$ & 0.76 & $(0.53-1.09)$ \\
\hline \multicolumn{8}{|l|}{$\underline{\text { Gender }}$} \\
\hline Female & 7 & 1.16 & $(0.52-2.58)$ & 0.97 & $(0.42-2.23)$ & 0.85 & $(0.36-1.98)$ \\
\hline Male & 31 & 0.87 & $(0.60-1.27)$ & 0.80 & $(0.54-1.19)$ & 0.77 & $(0.52-1.15)$ \\
\hline
\end{tabular}

$\wedge$ Because patients with a history of ischemic heart disease prior to the index date were excluded, the total number of AS patients was 3,640 and the total number of controls was 25,299

\# Because patients with a history of acute myocardial infarction prior to the index date were excluded, the total number of AS patients was 3,738 and the total number of controls was 25,843 The controls are used as reference group with a Hazard Ratio of 1.0

1) Adjusted for: age, gender, and the DDD of NSAID use

2) Adjusted for: age, gender, DDD of NSAID use, as well as smoking status, BMI, and use of antihypertensives, antiplatelets, antidiabetics, statins in the past 6 months.

AS, Ankylosing Spondylitis; Adj, adjusted; HR, hazard ratio; $\mathrm{Cl}$, confidence interval, NSAID, nonsteroidal anti-inflammatory drugs

Table 4: Patients with AS with or without recent NSAID use were compared with all controls, irrespective of the use of NSAIDs in the control group. The controls are the reference group with a Hazard ratio of 1.0.

1) HR adjusted for: age, gender, as well as smoking status, BMI, and use of antihypertensives, antiplatelets, antidiabetics, statins in the past 6 months.

2) HR adjusted for: age, gender, use of COX-II inhibitors and other traditional NSAIDs other than naproxen in the previous 3 months as well as smoking status, BMI, and use of antihypertensives, antiplatelets, antidiabetics, statins in the past 6 months.

3) HR adjusted for: age, gender, use naproxen and other traditional NSAIDs in the previous 3 months as well as smoking status, BMI, and use of antihypertensives, antiplatelets, antidiabetics, statins in the past 6 months.

4) HR adjusted for: age, gender, use of naproxen and COX-II inhibitors in the previous 3 months as well as smoking status, BMI, and use of antihypertensives, antiplatelets, antidiabetics, statins in the past 6 months.

AS, Ankylosing Spondylitis; IHD, ischemic heart disease; HR, hazard ratio; $\mathrm{Cl}$, confidence interval, NSAID, non-steroidal anti-inflammatory drugs; COX-2 inhibitors, Cyclooxygenase-2 inhibitor 


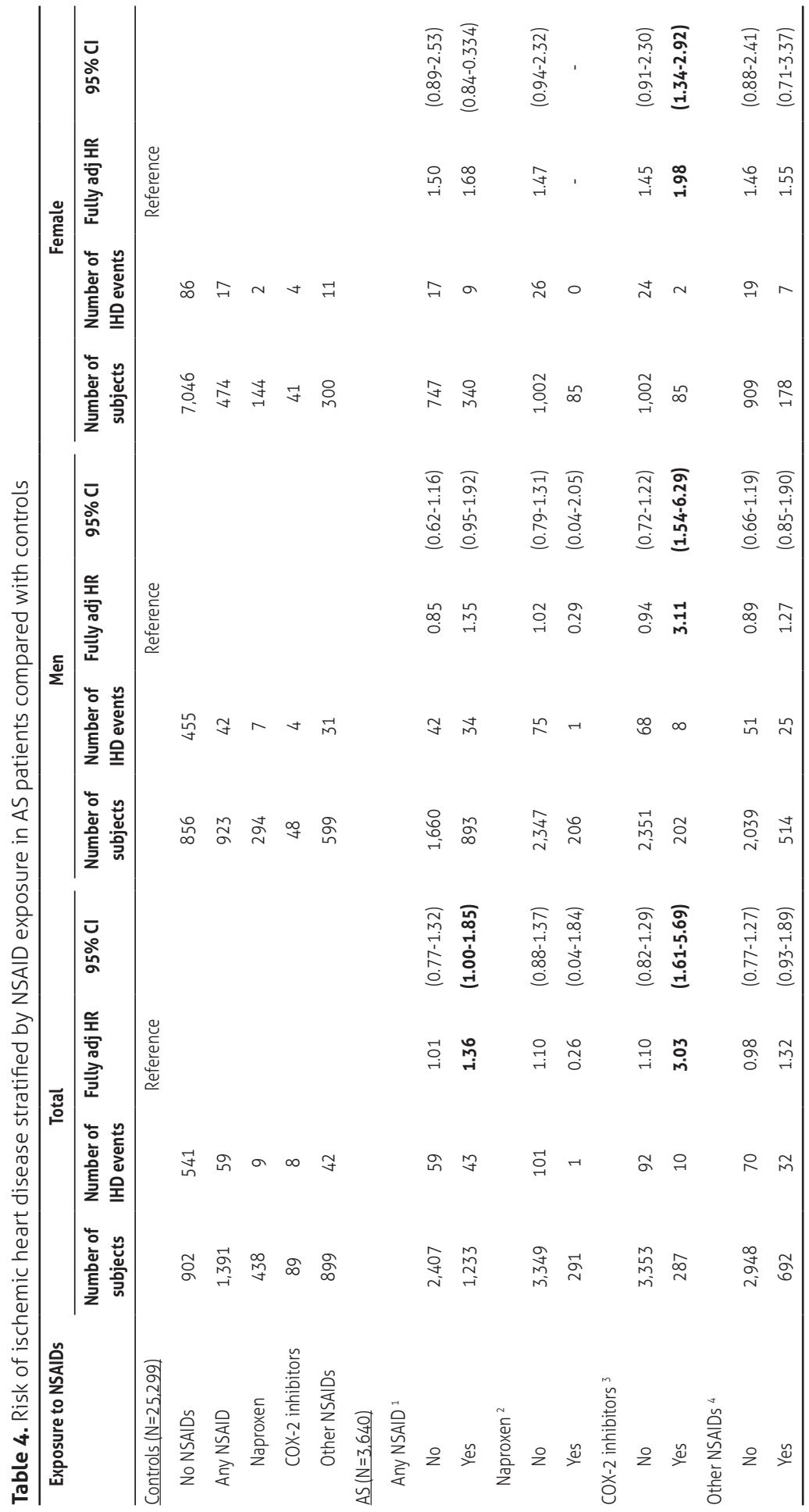


95\%-Cl 1.22-2.90), but it was no longer significantly increased after adjustment for the DDD of NSAIDs use (adj HR1.57, 95\%-CI 0.99-2.48). In addition, Table 4 shows that the risk of IHD in patients with AS was 1.4-fold increased with recent use of NSAIDs (fully adj. HR 1.36, 95\% Cl 1.00-1.85), and 3.0-fold increased with recent use of COX-2 inhibitors (fully adj HR 3.03,95\%-Cl 1.61-5.69) compared with all controls irrespective of their NSAID use. Moreover, patients with AS using COX-2 inhibitors had an increased risk of IHD compared with patients who did not use this drug (data not shown).

\section{DISCUSSION}

The present study investigated the incidence and risk of IHD and AMI, including the role of NSAIDs, in patients with AS compared with population-based matched controls. While the incidence of IHD was not increased in male patients, a significant increase was found in female patients compared with controls. After adjustment for NSAIDs use, however, only a non-significant trend towards increased risk of IHD in females was found. Recent use of NSAIDs, and in particular COX-2 inhibitors, resulted in a 1.4-fold and 3.0-fold fully adjusted overall risk of IHD in patients with AS compared with controls. An increased risk of AMI could not be demonstrated, and no gender differences were found.

In the literature, an increased risk of IHD in patients with AS has been reported earlier. Claims data from the Canadian province Quebec showed increased IHD risks in 4,836 male patients of all age categories (prevalence ratio ranging from 1.17 to 1.75 ) and in 3,169 female patients younger than 60 years (prevalence ratio ranging from 1.54 to 1.97), but not in 701 female patients older than 60 years (prevalence ratio $1.08,95 \%-\mathrm{Cl}$ 0.99-1.17) compared with a general population cohort [9]. Two other population-based studies, not stratified for gender, reported an increased risk of IHD in patients with AS after adjustment for age and gender ( $N=935$, standardized morbidity ratio $2.20,95 \%$ $\mathrm{Cl}$ 1.27-2.70 [7]), and after adjustment for hypertension and hyperlipidemia only ( $\mathrm{N}=$ 4.794, HR $1.4795 \%-C l 1.13$ to 1.92) [16]. Various explanations can be found for the differences between reported risks and our results. Although sample sizes were large and comparable, our study included only newly diagnosed patients with AS, whereas other studies included both prevalent and incident patients. At baseline, we found a higher prevalence of IHD in patients with AS compared with controls, which is in line with a cross-sectional study, which also found a 1.5 higher relative risk (95\%-Cl 1.0-1.5) of IHD in patients with AS compared with matched controls [8]. Furthermore, in contrast to other studies, we were able to statistically adjust for a wide range of confounders, including the use of NSAIDs. In this study, we demonstrated that use of NSAIDs explained a substantial proportion of the association between AS and risk of IHD. Our results are in line with the growing evidence that also COX-2 inhibitors may increase the risk of IHD 
$[6,22]$. In general, NSAIDs inhibit the activity of both COX-1 (thromboxane; thrombogenic and arthrogenic) and COX-2 activity (prostacyclin; opposes thromboxane). A COX-2 inhibitor--associated disruption of this balance might increase the risk of atherosclerosis, thrombogenesis, and CV complications [22].

A broad definition of IHD was used in this study, consisting of several of ischemic heart conditions and symptoms, among which angina pectoris. It is possible that misdiagnosis has occurred, because chest pain may also be caused by other conditions. Therefore, a more 'reliable' measure for IHD, i.e. AMI, which was diagnosed on objective findings on electrocardiogram and blood abnormalities, was investigated. Earlier studies reported conflicting results with respect to the risk of AMI in AS. Two population-based studies with respectively 935 and 1,686 patients with AS, failed to demonstrate an increased risk of developing $\mathrm{AMI}[7,14]$, which is similar to our results. In contrast, a cross-sectional survey from the Netherlands among 383 patients with AS (age 50-75 years) under the care of a rheumatologist reported a 3.1-fold increased risk of AMI (95\%-Cl 1.89-5.09) compared with patients selected from a general practitioner database [12]. The association in this cross-sectional survey is probably largely explained by information bias due to differential recording of exposure and outcome between both cohorts: the quality of AS recording has not been validated and there is clear evidence that AMI is substantially under recorded [15].

Interestingly, we found a difference in the IHD risk between female and male patients, but we could not show this difference for the AMI risk. A possible explanation might be that female patients suffer more from enthesitis [23] and widespread ('fibromyalgia-like') pain [24] compared with male patients, which might be misdiagnosed as IHD.

Some limitations of the present study need to be addressed. First, an association between AS and IHD or AMI may have been masked by non-differential misclassification of exposure (AS, NSAID use) or outcome (IHD, AMI). Linkage of a different regional UK GP dataset with a rheumatology registry showed that $24 \%$ of the patients with an AS diagnoses were not captured by the GP [14]. We do not have information on this within CPRD. In addition, a recent study showed a $25 \%$ under recording rate of AMI in CPRD [25]. Furthermore, information on over-the-counter use of NSAIDs was lacking, which could have resulted in misclassification of NSAID exposure, however, it is difficult to quantify the degree of misclassification. The second limitation is our operational definition of 'incident' patients with AS. A proportion of the patients may have suffered from AS for a longer time, either because of a delay in diagnosis, or because the first diagnostic code for AS in CPRD did not correspond with the actual diagnostic date of AS. Third, the positive associations that we have reported may also be explained by diagnostic bias. Patients with AS may have visited their health care provider more regularly because of their disease, and as such IHD and AMI may have been earlier or more frequently diagnosed compared with controls. Furthermore, as above described, chest pain is a feature of AS, and may therefore 
be misclassified as IHD, which can also explain the different findings with the lack of association between AS and AMI. Fourth, we did not have information on patient disease characteristics including HLA-B27 status, physical activity, disease activity and prescriptions of biologicals. Inflammation might accelerate the progress of atherosclerosis, and it is uncertain whether biologicals have a beneficial effect on subclinical atherosclerosis in AS [26]. Moreover, NSAID use might be a surrogate for disease activity, because patients with a higher disease activity are more likely to use NSAIDs.

Strengths of this study are the large sample size, and substantial duration of follow-up. CPRD is representative for the total UK population. In contrast to most previous studies, we had a population-based comparison group which was randomly selected from CPRD. In addition, we were the first study that could statistically adjust for a wide range of potential confounders, including smoking status, BMI, comorbidities, and the use of comedications including NSAIDs.

In conclusion, this study showed that female patients with AS seemed to be at an increased risk of developing IHD compared with female population-based controls in an age adjusted analysis, but after adjustment for recent NSAID use there was only a trend towards increased risk. There was no increased risk of AMI in patients with AS compared with controls. Although it cannot be excluded that NSAID use is (partly) a reflection of disease activity, rheumatologists should carefully balance the beneficial effects of NSAIDs and the increased risk of IHD in patients with AS. 


\section{REFERENCES}

1. Murray CJ, Lopez AD. Measuring the global burden of disease. N Engl J Med 2013;369:448-57.

2. Maradit-Kremers H, Nicola PJ, Crowson CS, et al. Cardiovascular death in rheumatoid arthritis: a populationbased study. Arthritis Rheum 2005;52:722-32.

3. Solomon DH, Karlson EW, Rimm EB, et al. Cardiovascular morbidity and mortality in women diagnosed with rheumatoid arthritis. Circulation 2003;107:1303-7.

4. Peters MJ, Symmons DP, McCarey D, et al. EULAR evidence-based recommendations for cardiovascular risk management in patients with rheumatoid arthritis and other forms of inflammatory arthritis. Ann Rheum Dis 2010;69:325-31.

5. Perk J, De Backer G, Gohlke H, et al. European Guidelines on cardiovascular disease prevention in clinical practice (version 2012). The Fifth Joint Task Force of the European Society of Cardiology and Other Societies on Cardiovascular Disease Prevention in Clinical Practice (constituted by representatives of nine societies and by invited experts). Eur Heart ] 2012;33:1635-701.

6. Mathieu S, Motreff $P$, Soubrier M. Spondyloarthropathies: an independent cardiovascular risk factor? Joint Bone Spine 2010;77:542-5.

7. Bremander A, Petersson IF, Bergman S, et al. Population-based estimates of common comorbidities and cardiovascular disease in ankylosing spondylitis. Arthritis Care Res (Hoboken) 2011;63:550-6.

8. Han C, Robinson DW, Jr., Hackett MV, et al. Cardiovascular disease and risk factors in patients with rheumatoid arthritis, psoriatic arthritis, and ankylosing spondylitis. J Rheumatol 2006;33:2167-72.

9. Szabo SM, Levy AR, Rao SR, et al. Increased risk of cardiovascular and cerebrovascular diseases in individuals with ankylosing spondylitis: a population-based study. Arthritis Rheum 2011;63:3294-304.

10. Mathieu S, Gossec L, Dougados M, et al. Cardiovascular profile in ankylosing spondylitis: a systematic review and meta-analysis. Arthritis Care Res (Hoboken) 2011;63:557-63.

11. Peters MJ, van der Horst-Bruinsma IE, Dijkmans BA, et al. Cardiovascular risk profile of patients with spondylarthropathies, particularly ankylosing spondylitis and psoriatic arthritis. Semin Arthritis Rheum 2004; 34:585-92.

12. Peters MJ, Visman I, Nielen MM, et al. Ankylosing spondylitis: a risk factor for myocardial infarction? Ann Rheum Dis 2010;69:579-81.

13. Sukenik S, Pras A, Buskila D, et al. Cardiovascular manifestations of ankylosing spondylitis. Clin Rheumatol 1987:6:588-92.

14. Brophy S, Cooksey R, Atkinson M, et al. No increased rate of acute myocardial infarction or stroke among patients with ankylosing spondylitis-a retrospective cohort study using routine data. Semin Arthritis Rheum 2012;42:140-5.

15. de Vries F AKV. Ankylosing spondylitis and myocardial infarction: a true association or selection bias? Annals of Rheumatic Diseases 2010.

16. Huang YP, Wang YH, Pan SL. Increased risk of ischemic heart disease in young patients with newly diagnosed ankylosing spondylitis--a population-based longitudinal follow-up study. PLoS One 2013;8: e64155.

17. Bhala N, Emberson J, Merhi A, et al. Vascular and upper gastrointestinal effects of non-steroidal antiinflammatory drugs: meta-analyses of individual participant data from randomised trials. Lancet 2013; 382:769-79.

18. Agency EM. New safety advice for diclofenac. New measures aim to minimise cardiovascular risks. 2013 [updated 25 september; cited 201310 December]; Available from: http://www.ema.europa.eu/docs/ en_GB/document_library/Referrals_document/Diclofenac-containing_medicinal_products/European_ Commission_final_decision/WC500155819.pdf.

19. McGettigan P, Henry D. Cardiovascular risk and inhibition of cyclooxygenase: a systematic review of the observational studies of selective and nonselective inhibitors of cyclooxygenase 2. Jama 2006;296: 1633-44. 
20. Herrett E, Thomas SL, Schoonen WM, et al. Validation and validity of diagnoses in the General Practice Research Database: a systematic review. Br ] Clin Pharmacol 2010;69:4-14.

21. WHO C, Centre, for, Drug, Statistics, Methodology. ATC/DDD Index 2014. 2014 [updated 2013-12-19; cited 2014 2nd october]; Available from: http://www.whocc.no/atc_ddd_index/.

22. Fosslien E. Cardiovascular complications of non-steroidal anti-inflammatory drugs. Ann Clin Lab Sci 2005; 35:347-85.

23. Tournadre A, Pereira B, Lhoste A, et al. Differences between women and men with recent-onset axial spondyloarthritis: results from a prospective multicenter French cohort. Arthritis Care Res (Hoboken) 2013;65: 1482-9.

24. Slobodin G, Reyhan I, Avshovich N, et al. Recently diagnosed axial spondyloarthritis: gender differences and factors related to delay in diagnosis. Clinical rheumatology 2011;30:1075-80.

25. Herrett E, Shah AD, Boggon R, et al. Completeness and diagnostic validity of recording acute myocardial infarction events in primary care, hospital care, disease registry, and national mortality records: cohort study. Bmj 2013:346:f2350.

26. Tam LS, Shang O, Kun EW, et al. The effects of golimumab on subclinical atherosclerosis and arterial stiffness in ankylosing spondylitis--a randomized, placebo-controlled pilot trial. Rheumatology (Oxford) 2014. 


\section{CHAPTER 8}

\section{Infliximab treatment reduces depressive symptoms in patients with ankylosing spondylitis: a subgroup analysis of a randomized, placebo-controlled trial (ASSERT)}

Carmen Stolwijk, Olga Schiepers, Thea Schoonbrood, Astrid van Tubergen, Désirée van der Heijde, Robert Landewé, Annelies Boonen 


\section{ABSTRACT}

\section{Objective:}

Depressive symptoms are common in patients with ankylosing spondylitis (AS). The objective of this study was to evaluate the effect of infliximab on depressive symptoms in patients with AS in a randomized-controlled trial setting.

\section{Methods:}

Data were retrieved from a subgroup of patients included in Maastricht, from the Ankylosing Spondylitis Study for the Evaluation of Recombinant Infliximab Therapy (ASSERT). Patients were randomly assigned to receive infliximab $(n=17)$ or placebo $(n=6)$ until week 24 after which all patients continued with infliximab until week 54. Depressive symptoms were measured with the Center for Epidemiological Studies Depression scale (CES-D, range 0-60) at weeks $0,6,12,24$, and 54.

\section{Results:}

The average CES-D depression score at baseline was 15.2 (SD 9.0) in the infliximab group and 16.8 (SD 5.4) in the placebo group ( $p=0.69$ ). $47.8 \%$ had a CES-D score $\geq 16$, indicative of clinical depression. After 24 weeks, mean CES-D scores had decreased to 10.8 (SD 11.4 ) in the infliximab group, and was still 16.2 (SD 6.8) in the placebo group. Generalized estimating equations for the first 24 weeks confirmed a trend of a different course of depression between groups $(p=0.06)$. In the subgroup of patients with depression at baseline, improvements in depression scores correlated moderately with improvements in BASDAI $(r=0.76)$ and with BASFI $(r=0.74)$ after 24 weeks of infliximab treatment.

\section{Conclusion:}

Infliximab improved symptoms of depression in patients with AS when compared to placebo. 


\section{INTRODUCTION}

Depressive symptoms are an important aspect of health related quality of life and often occur as comorbidity in patients suffering from chronic diseases. A limited number of studies in patients with ankylosing spondylitis (AS) reported an increased prevalence of depressive symptoms, ranging between $14.8 \%$ and $55.5 \%$ according to different screening questionnaires [1-5]. Also when using a doctor-diagnosis of depression for case confirmation, a recent study confirmed that the prevalence of depression was increased by more than $60 \%$ in patients with AS compared with the general population [6].

Studying depression or depressive symptoms in patients with AS is challenging. First, different pathophysiologic pathways might explain the increased prevalence of depressive symptoms in patients with AS. An obvious explanation could be that depressive symptoms are secondary to disease-related pain, impairments, worries or changes in self-esteem $[1,3,7]$. On the other hand, AS and depressive symptoms may share a common pathophysiology and may be both the result of the inflammatory immune response. It has been found that pro-inflammatory cytokines, such as TNF-a, were higher in patients with acute depression than in subjects without depression [8]. Several pathways have already been identified that can explain an association between inflammation and the pathophysiology of depression. For example, cytokines might activate the neuronal serotonin transporter and the tryptophan- and serotonin-degrading enzyme indolamine2,3-dioxygenase, both resulting in serotonin deficiency, which is associated with depression. Further, cytokines may activate the hypothalamic-pituitary adrenal axis system [8]. Likely, differences in susceptibility for the different mechanisms exist between different persons [9].

A second challenge is that the instruments used to assess depressive symptoms (or criteria to classify depression) are partly based on manifestations that are also manifestations of the disease such as tiredness or poor sleep. As such, the direct impact of the disease will be reflected in the questionnaires, which hampers unravelling whether depressive symptoms are a direct consequence of the disease manifestations or caused by pathways independent of pain and stiffness.

A clinically relevant question which eventually might also help in unraveling the role of TNF-a itself is the discussion whether anti-TNF-a agents could reduce depressive symptoms in patients with AS. Several randomized controlled trials (RCT) have shown that anti-TNF-a therapy improved symptoms of depression in patients with psoriasis and inflammatory bowel disease (IBD), both partly independently of disease activity [10,11]. Only one study among 16 patients with AS showed that depression scores decreased significantly after infliximab. However, this study was not randomized, and could therefore not exclude that the improvement in depression scores was a result of natural fluctuations of the diseases [12]. 
The objectives of the present study were to:

1) assess the effect of infliximab on depressive symptoms in patients with AS compared to placebo treated patients

2) explore the association between depressive symptoms and disease activity, at baseline and over time.

\section{METHODS}

Data were retrieved from a sub-study of the Ankylosing Spondylitis Study for the Evaluation of Recombinant Infliximab Therapy (ASSERT), and more specifically from the patients that were included at the Maastricht University Medical Center and who completed a questionnaire on depressive symptoms in addition to the protocol required assessments. The study population consisted of 23 patients, of whom 16 patients were randomly assigned to infliximab and 7 patients to placebo.

\section{Study design}

The design of the ASSERT trial has been previously reported [13]. In brief, patients were included in this multicenter RCT if they were 18 years or older and classified as having AS (according to the modified New York criteria) for at least 3 months prior to screening. Patients were required to have a Bath AS Disease activity index (BASDAI) score $\geq 4$ and a spinal pain assessment score of $\geq 4$ on a visual analog scale (VAS). Patients were randomly assigned to receive infusions of placebo or $5 \mathrm{mg} / \mathrm{kg}$ infliximab at weeks $0,2,6$, 12, and 18. From week 24 until week 54, all patients received infliximab therapy.

\section{Study outcomes}

Presence of depressive symptoms was assessed with the Center for Epidemiologic Studies Depression scale (CES-D) [14]. This instrument was chosen since it contains fewer somatic items than many other instruments $[1,15]$. The CES-D (range 0 to 60 ) consists of 20 items on perceived mood and level of functioning during the past week. Every item is scored on a 4-point scale, where $0=$ rarely or none of the time, $1=$ some or little of the time, 2 =occasionally or a moderate amount of time, and $3=$ most of the time. A CES-D score of $\geq 16$ is employed as a cutoff for clinical depression and usually warrants a referral for a more thorough diagnostic evaluation. Disease activity and physical function were measured with the BASDAI and Bath AS Functional Index (BASFI), respectively [16, 17]. Study outcomes were assessed at weeks $0,6,12,24$, and 54. 


\section{Statistical analysis}

Chi-square tests and Mann-Whitney $U$ tests were used to respectively compare the number of patients with a CES-D score $\geq 16$ and the mean CES-D scores between groups at the different time points. Individual course of CES-D scores between groups over time were compared using generalized estimations equations (GEE) analyses of covariance, controlling for baseline values of CES-D. Spearman correlation coefficients were used to understand the association 1 ) between depression scores and disease activity at baseline, 2) and changes in depression scores and changes in disease measures among patients with depression at baseline who received infliximab, because improvement in depression scores can only be expected in these patients. Correlation coefficients 0.2 to 0.5 were qualified as weak, 0.5 to 0.8 as moderate, and 0.8 to 1.0 as strong [18].

\section{RESULTS}

All patients ( $n=24$ ) completed the first 24 weeks of the study, and 23 patients the total 54 weeks. A CES-D score was missing in weeks 6, 24, and 54, but always for a different patient. At baseline, the groups were comparable in terms of demographics and disease characteristics (table 1). Most were men (78\%), the mean age was 40.4 years, and the mean disease duration was 9.3 years. Patients had active disease, with a mean BASDAI of 7.5. The mean CES-D score at baseline was 15.2 (SD 0.9) in the infliximab group and 16.8 (SD 5.4) in the placebo-group ( $p=0.69$ ). Fifty percent ( 8 of 16 ) of the patients in the infliximab group and $42.9 \%$ (3 of 7 ) in the placebo-group had a CES-D score $\geq 16$, indicative for clinical depression.

The mean CES-D score at week 6 had decreased to 9.9 (SD 7.4) in the infliximab group and was 15.9 (SD 6.0) in the placebo-group ( $p=0.03$ ) (figure 1). At 24 weeks, mean CES-D scores were 10.8 (SD 11.4) in the infliximab group and 16.2 (SD 6.8) in the placebo group $(p=0.07)$. At week 54, when the original placebo group had switched to infliximab, the mean CES-D score was decreased to the same degree as the infliximab group to 8.5 (SD 13.0). GEE analyses of covariance showed a trend for a difference between the infliximab and placebo groups for CES-D scores in the first 24 weeks $(p=0.06)$.

At week $6,25 \%$ ( 4 of 16 ) of the infliximab group had a CES-D score $\geq 16$ indicative for clinical depression, compared to 50\% (3 of 6) in the placebo group ( $p=0.26)$. After 24 weeks, these proportions were $20 \%$ (3 of 15 ) and $57 \%$ ( 4 of 7$)$, respectively $(p=0.17)$. At week 54, when all patient received infliximab, 20\% (1 of 5) of the original placebo group, had a CES-D score $\geq 16$.

At baseline, the CES-D score did not significantly correlate with BASDAI $(r=0.03, p=0.87)$ and BASFI $(r=0.05, p=0.82)$ scores. In patients with depression at baseline who received infliximab, week 6 changes in CES-D scores were weakly correlated with changes in 
Table 1. Baseline characteristics separately for patients in the infliximab and placebo arm*

\begin{tabular}{lccc}
\hline & Infliximab (n=16) & Placebo (n=7) & P-value \\
\hline Men, no. (\%) & $11(68.8)$ & $7(100)$ & 0.12 \\
Age, years & $38.6(11.6)$ & $44.9(5.8)$ & 0.19 \\
Disease duration, years & $8.3(8.2)$ & $11.5(7.4)$ & 0.38 \\
HLA-B27 positive, no. (\%) & $14(87.5)$ & $5(71.4)$ & 0.35 \\
History of uveitis, no. (\%) & $6(37.5 \%)$ & $1(14.3 \%)$ & 0.27 \\
History of psoriasis, no (\%) & 0 & 0 & - \\
History of IBD, no (\%) & $2(12.5 \%)$ & $1(14.3 \%)$ & 0.91 \\
BASDAl score, 0-10 & $7.0(1.3)$ & $7.1(0.7)$ & 0.68 \\
BASFI score, 0-10 & $6.3(1.5)$ & $7.2(1.3)$ & 0.21 \\
Patient's global assessment, 0-10 VAS & $6.8(1.4)$ & $7.8(1.2)$ & 0.14 \\
Mander entheses index, 0-90 & $7.7(8.6)$ & $12.8(5.5)$ & 0.30 \\
Swollen joint index, 0-44 & $2.9(3.7)$ & $3.0(3.9)$ & 0.97 \\
Chest expansion & $2.0(0.9)$ & $2.5(2.0)$ & 0.51 \\
Night pain, 0-10 VAS & $6.3(2.0)$ & $7.4(1.0)$ & 0.22 \\
CRP level, mg/dl & $2.6(2.4)$ & $1.6(1.6)$ & 0.31 \\
Screening CRP level, $\leq 3$ times ULN**; no (\%) & $6(37.5)$ & $5(71.4)$ & 0.13 \\
Screening CRP level, > 3 times ULN**, no (\%) & $10(62.5)$ & $2(28.6)$ & 0.13 \\
CES-D score, 0-60 & $15.2(9.0)$ & $16.8(5.4)$ & 0.69 \\
CES-D $\geq 16$, no. (\%) & $8(50 \%)$ & $3(42.9 \%)$ & 0.75 \\
\hline
\end{tabular}

* Values are mean (SD), unless otherwise indicated. HLA-B27= human leucocyte antigen-B27; IBD= inflammatory bowel disease; BASDAI= Bath Ankylosing Spondylitis Disease Activity Index; BASFI= Bath Ankylosing Spondylitis Functional Index; VAS= visual analog scale; $C R P=C$-reactive protein; ULN= upper limit of normality; $C E S-D=$ Center for Epidemiologic Studies Depression Scale. ** Normal range $0.05 \mathrm{mg} / \mathrm{dl}$

BASDAI ( $r=0.49, p=0.22)$ and strongly with BASFI $(r=0.90, p<0.01)$. At week 24, changes in CES-D scores were moderately related to changes in BASDAI scores $(r=0.76, p=0.03)$ and BASFI scores $(r=0.74, p=0.04)$. Visual exploration of the changes in CES-D and BASDAI showed relatively earlier improvement in CES-D scores (at week 6) compared with BASDAI scores in a part of the patients (results not shown).

\section{DISCUSSION}

This study showed that depressive symptoms are very common in patients with AS and high disease activity. Infliximab improved symptoms of depression in patients with AS. The greatest improvement was observed 6 weeks after start of treatment.

Almost forty-eight percent of the patients with AS and active disease had a CES-D score $\geq 16$ at baseline. This proportion is in the higher range of reported prevalences of depressive symptoms in patients with AS compared with other studies (15\%-55\%) [1-4]. 

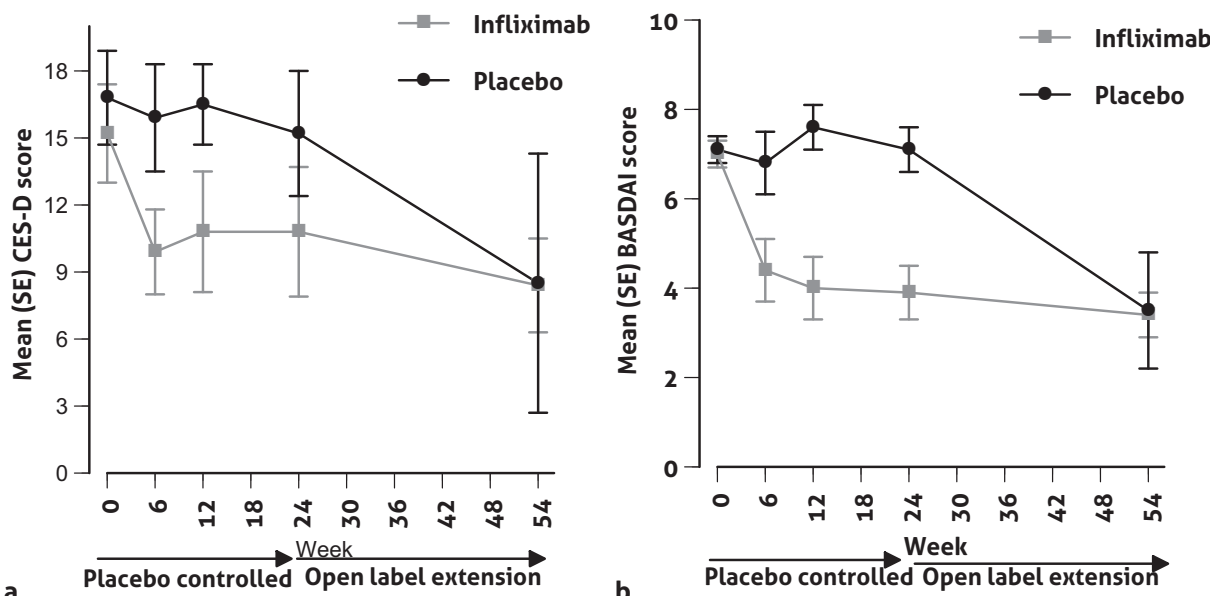

Figure 1 - CES-D (a) and BASDAI (b) scores over time according to treatment group. During the open-label extension, all patients received infliximab." Values are mean (SD), unless otherwise indicated. HLA-B27 = human leucocyte antigen-B27; IBD= inflammatory bowel disease; BASDAI= Bath Ankylosing Spondylitis Disease Activity Index; BASFI= Bath Ankylosing Spondylitis Functional Index; VAS= visual analog scale; $C R P=C$-reactive protein; $U L N=$ upper limit of normality; $C E S-D=$ Center for Epidemiologic Studies Depression Scale. ** Normal range 0.05 mg/dl

This may be a result of the selection of patients with high disease activity. While all patients had high BASDAI and BASFI scores at baseline, only a subgroup of these patients had CES-D scores above the threshold for clinical depression. It would be interesting to further explore which patients with active disease have an increased susceptibility to depression, for example as a result of genetic characteristics or personality traits.

In patients with a CES-D score $\geq 16$ at baseline, the improvement in depression over 24 weeks was moderately related to improvement in disease activity and physical function. This suggests that improvement in disease activity only partly explains the improvement in depressive symptoms. Interestingly, the improvement in depressive symptoms seemed to precede the improvement in BASDAI in a part of the patients. If the depressed mood was merely secondary to disease activity, one would expect BASDAI to decrease faster, while this was not clearly seen.

The main limitation of this study is the small number of patients restricting the power to detect significant changes and to perform complex statistical analyses. However, strong absolute changes and improvements in depressive symptoms at both the group- and the patient-level were observed. Further, we used the CES-D to assess depressive symptoms and we should realize that the CES-D is a screening questionnaire and does not allow making a diagnosis of depression, for which the gold standard is psychiatric interviewing and examination. Studies including expert opinion on depression and assessing more specific biological markers of depression pathways are warranted to evaluate the possible direct role of inflammation. 
The findings in the present study have several implications. Rheumatologists should be aware of the high prevalence of depressive symptoms in patients with AS and active disease, while considering that these symptoms are not strictly a result of pain and function loss. On this line, it can be questioned whether rheumatologists should take depressive symptoms into account when considering a new pharmacological treatment. In this light, it may be interesting to understand whether the impact of other medications such as NSAIDs or new classes of biologicals such as anti-IL-17 therapy would have similar effects on depressive symptoms.

In conclusion, the prevalence of depressive symptoms was high among this AS patient population. Anti-TNF-a treatment improved the depressive symptoms of AS patients; our data suggest that the benefit seems in part a direct effect of anti-TNF-a treatment. 


\section{REFERENCES}

1. Barlow JH, Macey S], Struthers GR. Gender, depression, and ankylosing spondylitis. Arthritis Care Res 1993; 6:45-51.

2. Gunaydin R, Goksel Karatepe A, Cesmeli N, Kaya T. Fatigue in patients with ankylosing spondylitis: relationships with disease-specific variables, depression, and sleep disturbance. Clin Rheumatol 2009;28: 1045-51.

3. Hakkou J, Rostom S, Aissaoui N, et al. Psychological status in Moroccan patients with ankylosing spondylitis and its relationships with disease parameters and quality of life. ] Clin Rheumatol 2011;17:424-8.

4. Hyphantis T, Kotsis K, Tsifetaki N, et al. The relationship between depressive symptoms, illness perceptions and quality of life in ankylosing spondylitis in comparison to rheumatoid arthritis. Clin Rheumatol 2013; 32:635-44.

5. Baysal O, Durmus B, Ersoy Y, et al. Relationship between psychological status and disease activity and quality of life in ankylosing spondylitis. Rheumatol Int 2011;31:795-800.

6. Meesters ], Bremander A, Bergman S, et al. The risk for depression in patients with ankylosing spondylitis: a population-based cohort study. Arthritis Res Ther 2014;16:418.

7. Martindale J, Smith J, Sutton C], et al. Disease and psychological status in ankylosing spondylitis. Rheumatology (Oxford) 2006;45:1288-93.

8. Himmerich H, Fulda S, Linseisen J, et al. Depression, comorbidities and the TNF-alpha system. Eur Psychiatry 2008;23:421-9.

9. Levinson DF. The genetics of depression: A review. Biol Psychiat 2006;60:84-92.

10. Guloksuz S, Wichers M, Kenis G, et al. Depressive symptoms in Crohn's disease: relationship with immune activation and tryptophan availability. PLoS One 2013;8:e60435.

11. Tyring S, Gottlieb A, Papp K, et al. Etanercept and clinical outcomes, fatigue, and depression in psoriasis: double-blind placebo-controlled randomised phase III trial. Lancet 2006;367:29-35.

12. Ertenli I, Ozer S, Kiraz S, et al. Infliximab, a TNF-alpha antagonist treatment in patients with ankylosing spondylitis: the impact on depression, anxiety and quality of life level. Rheumatol Int 2012;32:323-30.

13. van der Heijde D, Dijkmans B, Geusens P, et al. Efficacy and safety of infliximab in patients with ankylosing spondylitis: results of a randomized, placebo-controlled trial (ASSERT). Arthritis Rheum 2005;52:582-91.

14. Radloff LS. The CES-D Scale: A Self-Report Depression Scale for Research in the General Population. Appl Psychol Meas 1977;1:385-401.

15. Blalock S], DeVellis RF, Brown GK, Wallston KA. Validity of the Center for Epidemiological Studies Depression Scale in arthritis populations. Arthritis Rheum 1989:32:991-7.

16. Garrett $\mathrm{S}$, Jenkinson T, Kennedy LG, et al. A new approach to defining disease status in ankylosing spondylitis: the Bath Ankylosing Spondylitis Disease Activity Index. J Rheumatol 1994;21:2286-91.

17. Calin A, Garrett S, Whitelock H, et al. A new approach to defining functional ability in ankylosing spondylitis: the development of the Bath Ankylosing Spondylitis Functional Index. ] Rheumatol 1994;21:2281-5.

18. Zou KH, Tuncali K, Silverman SG. Correlation and simple linear regression. Radiology 2003:227:617-22. 



\section{CHAPTER 9 General discussion}

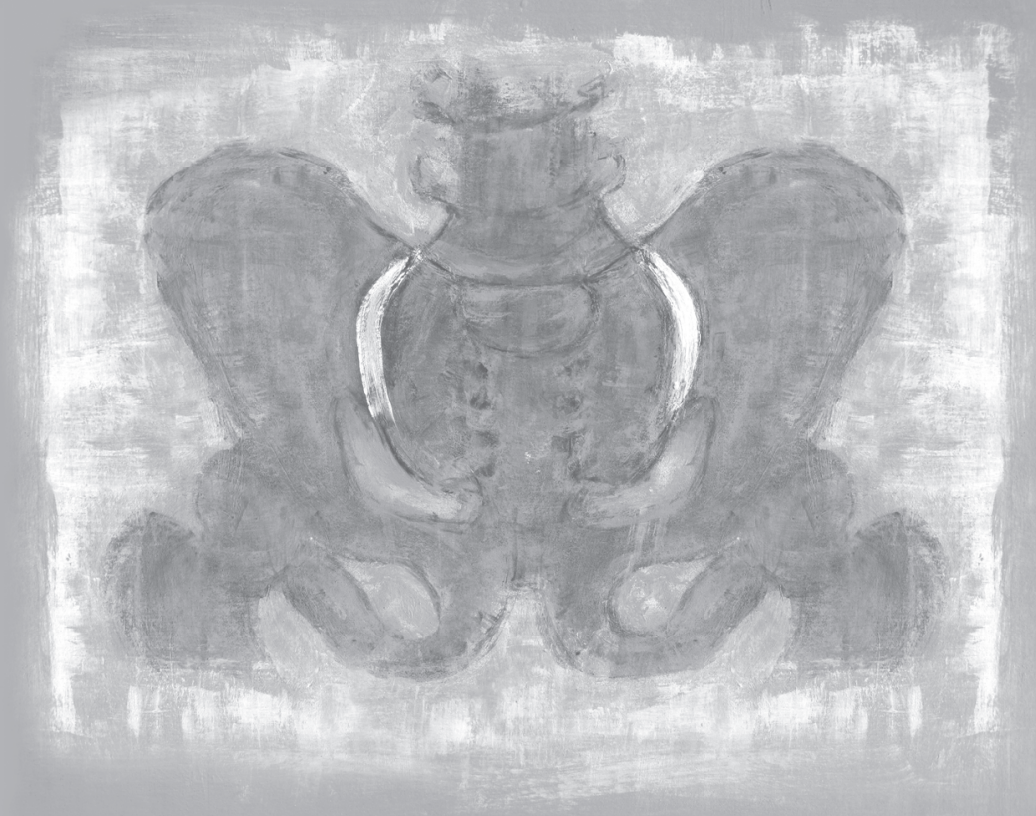





\section{GENERAL DISCUSSION}

In addition to axial and peripheral joints symptoms, many patients with SpA have at least one other chronic disorder, which may have consequences for the diagnosis, treatment, prognosis and outcome of their disease [1, 2]. In SpA, two types of co-existing conditions can be distinguished: 1) conditions which are related to the concept of SpA, the so called 'extra-articular manifestations' (EAMs) comprising acute anterior uveitis (AAU), psoriasis and inflammatory bowel disease (IBD), and 2) all other conditions that are not related to the SpA-concept but that may co-exist by chance or as a consequence of the disease process or its treatment, called 'comorbidities'.

In this thesis, several aspects of the EAMs and other co-existing diseases in patients with SpA are explored. More specifically our aims were: first, to summarize the available evidence on the epidemiology of SpA and explore the demographical and methodological factors related to the variation in reported prevalence rates in the literature; second, to gain insight into the epidemiology of EAMs in patients with ankylosing spondylitis (AS, which is the prototype of SpA), and vice versa into the frequency of spinal and articular SpA symptoms in patients with IBD (one of the three EAMs); third, to validate the selfadministered comorbidity questionnaire (SCO) for use in patients with AS; and finally, to improve our understanding about the frequency and risks of cardiovascular disease and depression in patients with AS.

Below, the overall findings of the different studies that were conducted are critically appraised from several perspectives and include nosological and methodological considerations, implications for clinical practice, and challenges for future research.

\section{Nosological considerations}

When considering the disorders outside the musculoskeletal system in patients with SpA, universal agreement on how to conceptualize and classify the broad range of manifestations that can co-exist with the spinal and articular disease is lacking. Moreover, and as explained below, we noticed a paradox in the conceptualization of the EAMs, and on how researchers and clinicians deal with these EAMs.

The term 'comorbidity' was introduced by Feinstein in 1970, who defined comorbidity as: 'any distinct additional entity that has existed or may occur during the clinical course of a patient who has the index disease under the study' [3]. Whether AAU, psoriasis, and IBD should be referred to as 'comorbidities' or not depends, according to this definition, on the question whether these manifestations are 'distinct' entities, or so closely related to the pathophysiology of SpA, that they are 'part of the index disease'.

From an epidemiological point of view we showed that AAU, psoriasis and IBD are much more prevalent in patients with AS than expected in population-based controls (chapter 3 and chapter 4). About $25 \%$ of patients with AS will develop at least one episode of 
AAU; $10 \%$ of patients suffer from psoriasis; and $5 \%$ of patients have IBD. Importantly, in about half of the patients this EAM develops before the diagnosis of AS. Compared with controls, the risk of AAU was 15 times increased in patients with AS, the risk of psoriasis was 1.5 times increased, and the risk of IBD was 3 times increased. As such, this high prevalence suggests a very close relation to the articular disease.

Further, from a pathophysiological view, since the description of the concept by Moll, there is accumulating evidence that all the different subtypes of SpA including the EAMs belong to a single disease-concept with a heterogeneous phenotype rather than that they are distinct disease entities [4]. This insight even applies to the heterogeneous articular manifestations, in which the pathophysiology of axial disease may be rather different from that of peripheral disease but still share similarities in the inflammatory pathways involved [4]. On these lines, the new Assessment of SpondyloArthritis international Society (ASAS) criteria classify axial and peripheral SpA as diseases with heterogeneous phenotypes $[5,6]$ and also include the presence of different EAMs as key elements of the classification criteria. It was shown that inclusion of the EAMs improved specificity of the criteria $[5,6]$. Both the epidemiological as well as the pathophysiological arguments suggest that the EAMs have actually become part of the 'index' disease and should not be referred to as 'comorbidities' because they are not 'distinct entities' when following the definition of Feinstein.

On the other hand, from the perspective of outcome research and clinical care, the different manifestations of SpA are usually considered as 'distinct additional entities', likely because this approach is more useful for diagnosis of individual patients, patient education, customized treatment, and prediction of outcome [7]. The articular symptoms and the EAMs are usually diagnosed and treated by different specialists, although some drugs may improve both. Also in light of outcome measurement, SpA and EAMs are usually considered as distinct entities. If we would consider the EAMs and articular manifestations as part of one disease, the EAMs should also be taken into account when measuring disease activity, physical function or disease-related quality of life. However, the core outcome domains and instruments that are proposed for outcome measurement in SpA focus solely on the articular manifestations of the disease. For example, the Ankylosing Spondylitis Disease Activity Score (ASDAS) developed for measuring disease activity and the Bath AS Functional Index (BASFI) for measuring physical function, include only items that relate to the articular features of the disease $[8,9]$. Instruments developed for measuring health related quality of life (HROoL), such as the AS Quality of Life (ASOLL) and ASAS Health Index (ASAS HI) seem somewhat more generic [10, 11]. However, it has been shown that the presence of EAMs was not associated with the ASOoL over time [12]. The ASDAS likely also does not capture the specific impact that IBD, AAU or psoriasis can have on patients with these co-existing diseases, although this should be further determined. 
In summary, when conceptualizing EAMs in SpA either as part of the disease concept or as comorbidities that concur with SpA, there is an inconsistency between etiopathogenic validity and clinical validity. From a research perspective it is definitely important to keep this distinction between EAMs and comorbidities in mind. From a perspective of clinical care, the high prevalence of EAMs requires attention from the different health care providers themselves.

\section{Methodological considerations}

This paragraph discusses several methodological considerations of the studies included in this thesis concerning the generalizability of the results and measurement of comorbidity.

\section{External validity}

The external validity refers to the generalizability of the study results to other populations. The external validity is strongly affected by in- and exclusion criteria used in a study. On this line, external validity can be affected by several other issues, such as setting (e.g. country, general practice or hospital sampled), characteristics of patients (e.g. severity of disease, comorbidity) and, in case of a trial, differences between the trial protocol and routine practice [13]. Strict in- and exclusion criteria increase the homogeneity of the study population leading to results which are valid for that subgroup of patients, but are less generalizable to the average patient in the general population. Broad sampling, on the other hand, results in a high generalizability of the findings, but this can be at the expense of interpretability of the results. Therefore, a balance needs to be reached between homogeneous and heterogeneous sampling and it will depend on the study type and the research question which approach is desirable.

In an observational study on disease frequency it is important to have a study sample that is representative for all (known and unknown) determinants of the disease. The Clinical Practice Research Datalink (CPRD) is a good example of such a highly generalizable population. CPRD is a population-based GP database and includes patients from all ages. It has been shown that the geographic distribution and demographic profile of CPRD is highly representative of the UK population [14]. In chapter 4, we studied in CRPD the prevalence, incidence and risk of EAMs in patients with AS compared to the general population. The AS population and the control population are expected to be representative of AS patients, including patients varying from mild to severe disease, and the general population respectively from the UK. Whether the data on increased risk of the EAMs can be generalized to other countries remains a question, especially since we confirmed in chapter 3 that the prevalence of EAMs is associated with geographic region, partly because of differences between HLA-B27. As a result, the pooled prevalence of the meta-analysis on the prevalence of EAMs, which included patients from all geographic 
regions, should be interpreted with caution and can most likely not be generalized to all AS patients. Similarly, the worldwide prevalence of SpA and its subtypes as presented in chapter $\mathbf{2}$ is difficult to interpret and region-specific pooled prevalence rates are preferred. A true worldwide prevalence would require that the different samples are representative for the different world-regions known to have a different epidemiology for SpA.

In a cause-effect study, on the other hand, the population does not have to be representative for all determinants of the disease, but only for the relation that is being studied. In contrast to differences in frequencies of diseases among different populations, it is less likely that cause-effects are different among different populations. However, this assumes no effect modification of the causal pathway, unless the effect modifiers are sample independent, which is hardly a valid hypothesis. Further, although relative effects may be comparable among different populations, absolute effects might differ. In chapter $\mathbf{5}$ we investigated the effect of AS on cardiovascular disease and the confounding effect of non-steroidal anti-inflammatory drugs (NSAIDs) on this risk in the CPRD database from the UK. It was concluded that the risk of ischemic heart disease was only higher in female patients with AS, but disappeared after adjustment for NSAIDs. After stratification for recent NSAID use, the risk of ischemic heart disease was only increased in patients who used an NSAID in the three months before the event. No increased risk in patients with AS was found with respect to myocardial infarction. The qualitative effect ('is there an effect?') of AS will probably not be different in patients from the UK compared with other countries, unless genetic or environmental factors modify this risk. The question remains, however, whether the results found in this study can be generalized to specific subgroups of AS patients. For example, the cardiovascular risk may also be higher for patients with severe AS compared to mild disease. In rheumatoid arthritis, it has been shown that disease activity was significantly associated with the development of cardiovascular disease [15]. In our study in AS we were not able to adjust for the influence of disease activity, but it cannot be excluded that NSAID use was in fact a surrogate marker for disease activity. Future studies are needed to investigate this.

In chapter 8, we studied the relation between AS and depression and the effect of antiTNF therapy on depressive symptoms. We showed that the prevalence of depressive symptoms was high in patients with high disease activity, but improved after start of anti-TNF therapy. Partly, depressive symptoms in patients with AS are related to pain and reduced physical function. Factors such as gender, genetic predisposition, and cultural background, however, may modify this relationship. As a consequence, the quantitative relation ('how strong is the effect') between AS and depression cannot simply be generalized to individual AS patients with other cultural or genetic background, or to patients with milder disease activity. 
In summary, generalizability of the prevalence of SpA and EAMs is particularly hampered by genetic differences in HLA-B27 among regions. These estimations should therefore be interpreted with caution. The relation between AS and both cardiovascular disease and depression is likely better generalizable (with regard to the question: 'is there a relation?'), although effect modification may play a role (especially with regard to the 'strength' of the relation), for example as a result of genetic or environmental differences.

\section{Measurement of co-existing disease}

When studying the occurrence or the importance of EAMs and comorbidity in relation to outcome, researchers need to be able to identify and/or measure EAMs and comorbidity. It depends on the research question and type of study, how comorbidity is preferably measured.

In epidemiological studies, particularly estimations on the prevalence or incidence of co-existing diseases is of interest. In etiologic studies, the different aspects of relationship between co-existing conditions and an index disease can be investigated. In these fields of research it is particularly useful to identify every disease as a separate variable and to gain insight into the relationship between the comorbidity and the index disease [16]. When the comorbidity is not very prevalent or when comorbidities occur in specific subgroups of patients only, large study populations are needed. Databases, such as CPRD, yield data for large groups of patients and may therefore be useful for this kind of research. In this thesis, we used the CPRD database to estimate the prevalence and incidence of EAMs in patients with AS compared with the general population (chapter 4), and to estimate the cardiovascular risk in patients with AS (chapter 7). Further, in the CPRD database, information on potential confounders, such as NSAID use, was available in order to investigate the etiological relationship between AS and cardiovascular disease. In CPRD, the case definition of comorbidities is based on read codes representing diagnoses entered by GPs during the clinical encounter. Although several studies have shown a high accuracy of these read codes, misclassification of the EAMS or comorbidities cannot be excluded [17]. For example, a diagnosis of angina pectoris might be made while the chest pain is in fact due to inflammation of the costosternal joints, and may have resulted in an overestimation of the cardiovascular risk in these patients. Interestingly, in chapter $\mathbf{3}$ we showed that the approach to define cases may influence the prevalence. The prevalence of AAU in patients with AS was significantly higher in studies based on self-report compared with medical record diagnoses (35.9\% for self-report versus $24.1 \%$ for medical records). In contrast, the prevalence rates of IBD and psoriasis were not significantly different with different case definitions, indicating that possible misclassification is dependent on the type of disease.

Results of randomized controlled trials (RCTs) and prognostic or outcome studies can also be influenced by the presence of a co-existing disease, which can either act as 
confounder, thereby threatening the internal validity, or as an effect modifier, thereby threatening the internal and external validity of the study. For these purposes a reliable method is needed to summarize and weight the co-existing diseases in one single score [16]. The content and scoring of such indices would be different, depending on the type of outcome that is of main interest. As such, the Charlson index specifically predicts mortality while the SCO or Rheumatic Disease Comorbidity Index (RDCI) (and many others) are intended for predicting functioning and HROoL $[18,19]$. Chronic diseases like SpA particularly influence physical function, and we confirmed that the SCO is preferred over the Charlson index when adjusting the impact of a disease for co-morbidities. In the SCQ, an extra question is added to each comorbidity to assess the impact of the specific condition on functioning which likely improved its construct validity. Further, instruments differ in the approach to measure comorbidity. The two most frequently used approaches are self-report questionnaires and medical chart review. The reliability of data from medical chart review depends on the completeness of the medical charts. The completeness of data obtained by questionnaires, on the other hand, depends on the ability of patients to adequately recall the diseases they suffer from. Previous studies have shown that self-report questionnaires are a good alternative method to predict OoL health outcomes compared to a medical record score [20]. Our study revealed that overall agreement between self-reported comorbidities and comorbidities retrieved from medical records was moderate to high for most diseases (chapter 6). Notwithstanding, differences were found across individual comorbid conditions. It seemed that more severe (e.g. myocardial infarction) or chronic diseases (e.g. diabetes mellitus) were more accurately reported compared with conditions that are less clearly defined and contain a subjective evaluation by the patient himself such as depression or stomach disease.

A specific challenge in SpA is whether EAMs should be included in a comorbidity index. In addition to the study question, the decision on whether or not to include the EAMs, also depends on the extent in which one expects that the outcome of interest on the one hand, or the other explanatory variables on the other hand already consider the EAMs. Since currently the influence of EAMs are not represented in disease-specific outcome instruments, such as the BASFI and the BASDAl, it seems reasonable to include the EAMs in a comorbidity index for specific use in SpA / AS when exploring outcomes. Whether (and for which outcomes) the construct and predictive validity of the SCO will improve after addition of the EAMs is currently tested in an ongoing study.

\section{Implications for research and research challenges}

Our studies answered several questions with regard to co-existing disease in SpA, EAMs as well as comorbidities, but also revealed research gaps that need further attention. With the new criteria of axial and peripheral SpA, the EAMs have now received also a more prominent role in the diagnosis of axial and peripheral SpA. However, there are 
still unanswered questions. Our epidemiological studies showed that AAU, psoriasis and $\mathrm{IBD}$ are more prevalent in patients with AS compared to the general population and also showed that the EAMs may develop either before or after the diagnosis of AS. However, the relation (over time) between articular manifestations and EAMs is still insufficiently understood. Etiopathogenetic studies that further unravel the pathogenesis of SpA and its relation with EAMs will be the key to our understanding, including the relation with HLA-B27 and possible gene-gene or gene-environment interactions. Further, we concentrated on AS. When accepting SpA as one disease concept, further cohorts should try to include and follow-up patients with the diverse spectrum of the disease in order to better understand similarities and differences in the expression of EAMs.

With respect to the classic comorbidities in AS (and SpA), we showed in a large and representative AS sample that the cardiovascular risk in patients with AS is not as high as often supposed, particularly after adjustment for possible confounders (chapter 7). The prevalence of depressive symptoms, on the other hand, was strikingly high in patients with severe AS (chapter 8 ). These findings suggest a gap in comorbidity research in AS: whereas nowadays many studies focus on the cardiovascular risk in several inflammatory rheumatic diseases, in AS it seems as important to further explore other consequences of the diseases, such as mental disorders. Of course, it remains of undebated importance that the role of medication, such as NSAIDs, in relation to the development of comorbidities, and more specifically their risk/benefit ratio, requires further attention. NSAIDs are highly effective in reducing symptoms of axial SpA (axSpA) and are considered as first line therapy [21]. It has been shown that continuous use of NSAIDs was associated with an inhibition of radiographic progression in the spine [22-24]. Continuous treatment with NSAIDs, however, raises safety issues. In line with previous studies in populations with diseases other than AS, we showed that part of the increased cardiovascular risk in patients with AS could be attributed to cyclo-oxygenase-2 (COX-2) inhibitors and nonselective NSAIDs (chapter 7). It will be a methodological challenge to investigate whether this is purely an effect of the NSAIDs or that NSAIDs are a surrogate for disease activity. While epidemiological studies can provide information on associations between disease activity and cardiovascular risk, these studies should be complemented by strictly controlled clinical studies to explore whether lowering disease activity with treat-to-target strategies would reduce the cardiovascular risk.

For studying the relationship between co-existing disease and outcome, we need instruments to measure comorbidity. EAMs and comorbidity are one of the most important confounders or effect modifiers in analyses on outcome in SpA with substantial impact on HROL $\mathrm{L}$ and functioning. Comorbidity scores should therefore be included in multivariable analyses on health outcomes in AS. The study presented in this thesis is the first step to investigate aspects of validity of the SCO in patients with AS (chapter 6). We proposed a modification of the SCO by removing the rheumatic items from the questionnaire, and 
adding an extra section on the EAMs. Further validation of this $\mathrm{mSCQ}$ is needed, including validation in other study populations, exploration of the potential improvement of the SCO by adding the EAMs and the association with the ASAS HI. This validation is now among others incorporated in the COMOrbidities in SPondyloArthritis study (COMOSPA), which is a large cross-sectional, international study evaluating the prevalence and patterns of co-morbidities in SpA patients. Further, this study provides information on both physician confirmed comorbidities and on self-reported comorbidities, as well as on outcome measures and demographic and clinical characteristics of the patients.

Our findings reveal also further questions on how to collect evidence that is used to inform clinical guidelines for healthcare delivery. Most clinical evidence and guidelines are largely created for individual diseases and most RCTs exclude patients with multimorbidity [25]. In more than $80 \%$ of the clinical trials, individuals are excluded from participation due to medical comorbidities [26]. The advantage of excluding patients with comorbidities is creating more homogeneous populations in whom an intervention has the greatest likelihood to produce a clinically important and statistically significant effect. Efficacy trials can therefore be smaller, shorter, more efficient, and less expensive [26]. However, patients who are likely to represent the population treated in clinical setting are excluded and results of efficacy trials often overestimate an intervention's effect in clinical practice. More effectiveness trials, which refer to trials in the 'real-world', are therefore needed as these are more relevant for health-care decisions by both providers in practice and policy-makers [27].

\section{Implications and recommendations for clinical practice}

The findings of the first part of this thesis as well as findings in the literature show that AS is frequently accompanied by EAMs and comorbidities. An EAM is already present before the diagnosis of AS in approximately half of the patients who ultimately develop an EAM. Primary goals nowadays with respect to axSpA are early recognition and diagnosis in order to provide early treatment, to control disease activity, to maintain physical function and to prevent radiographic progression [28]. Recognizing patients with possible axSpA and referral to a rheumatologist are still the main challenges nowadays. By including the EAMs in the classification criteria of SpA, their role in case definition is now formally recognized. It was shown that EAMs may be very useful in recognition of SpA patients and their presence increase specificity in the classification of SpA. Because the probability of axSpA in a patient with an EAM is high, ophthalmologist, dermatologists and gastroenterologists should actively ask for other SpA symptoms in their patients with $A A U$, psoriasis and IBD, respectively. We have shown that half of the patients with IBD who reported SpA symptoms were never referred to a rheumatologist (chapter $\mathbf{5}$ ). On the same line, GPs should be better educated to recognize disease patterns of SpA including axial symptoms, peripheral symptoms and EAMs. The presence of an EAM should 
increase the suspicion of SpA in a patient with chronic back pain or peripheral arthritis. For rheumatologists, the presence of an EAM may help in the diagnosis of SpA. The positive likelihood ratios for a diagnosis of SpA of AAU, psoriasis and IBD are 13.9 (5.5-35.2), 3.8 (2.5-5.8), and 4.3 (2.0-8.9), respectively [29].

This thesis further points to the significant impact of comorbidities on HRQoL, physical function and work disability (chapter 6). The care for patients with comorbidity asks for a patient-centered holistic approach, while in practice, a growing sub-specialization in medicine is seen. This fragmented care with uncoordinated prescriptions and recommendations may lead to polypharmacy, increased treatment costs, side effects and unintended drug interactions [30]. Evidence based guidelines provide disease specific guidance, but often fail to acknowledge the effects of comorbidity and/or EAMs and complexity. The ASAS recommendation on the treatment of AS includes different statements about EAMs and comorbidities: "the frequently observed EAMs should be managed in collaboration with the respective specialist"; "rheumatologists should be aware of the increased risk of osteoporosis and cardiovascular disease"; and "cardiovascular, gastrointestinal and renal risks should be taken into account when prescribing NSAIDs" [21]. While we further confirmed the cautions with regard to NSAID, we also showed that more attention is needed for depressive symptoms. Interestingly, however, no specific recommendations are given in the treatment guidelines with respect to screening for comorbidities in AS or treatment of AS in patients with multimorbidity. Comorbidity and EAMs should be acknowledged in clinical practice and more specific recommendations should be developed to deal with managing multiple chronic conditions. A patientcentered approach is needed instead of an approach that only focuses on the index disease, in particular for making treatment decisions and patient education. 


\section{REFERENCES}

1. Bremander A, Petersson IF, Bergman S, et al. Population-based estimates of common comorbidities and cardiovascular disease in ankylosing spondylitis. Arthritis Care Res (Hoboken) 2011;63:550-6.

2. van der Horst-Bruinsma IE, Nurmohamed MT, Landewe RB. Comorbidities in patients with spondyloarthritis. Rheum Dis Clin North Am 2012;38:523-38.

3. Feinstein A. The pre-therapeutic classification of co-morbidity in chronic disease. ] Chronic Dis 1970;23: 455-68.

4. Baeten $D$, Breban M, Lories $R$, et al. Are spondylarthritides related but distinct conditions or a single disease with a heterogeneous phenotype? Arthritis Rheum 2013;65:12-20.

5. Rudwaleit M, Landewe R, van der Heijde D, et al. The development of Assessment of SpondyloArthritis international Society classification criteria for axial spondyloarthritis (part I): classification of paper patients by expert opinion including uncertainty appraisal. Ann Rheum Dis 2009;68:770-6.

6. Rudwaleit M, van der Heijde D, Landewe R, et al. The Assessment of SpondyloArthritis International Society classification criteria for peripheral spondyloarthritis and for spondyloarthritis in general. Ann Rheum Dis 2011;70:25-31.

7. Zeidler $\mathrm{H}$. The historical concept of interrelated conditions grouped together as a family of distinct diseases is not outdated: comment on the article by Baeten et al. Arthritis Rheum 2013;65:2214-5.

8. Lukas C, Landewe R, Sieper ], et al. Development of an ASAS-endorsed disease activity score (ASDAS) in patients with ankylosing spondylitis. Ann Rheum Dis 2009;68:18-24.

9. Calin A, Garrett $S$, Whitelock $H$, et al. A new approach to defining functional ability in ankylosing spondylitis: the development of the Bath Ankylosing Spondylitis Functional Index. ] Rheumatol 1994;21:2281-5.

10. Doward LC, Spoorenberg A, Cook SA, et al. Development of the ASOoL: a quality of life instrument specific to ankylosing spondylitis. Ann Rheum Dis 2003;62:20-6.

11. Kiltz U, van der Heijde D, Boonen A, et al. Development of a health index in patients with ankylosing spondylitis (ASAS HI): final result of a global initiative based on the ICF guided by ASAS. Ann Rheum Dis 2014.

12. Essers I, Ramiro S, Stolwijk C, et al. Contribution of extra-articular manifestations to the burden of disease in ankylosing spondylitis: a longitudinal study (abstract). Ann Rheum Dis;73(Suppl2)2014.

13. Rothwell PM. External validity of randomised controlled trials: "to whom do the results of this trial apply?". Lancet 2005:365:82-93.

14. Lawson DH, Sherman V, Hollowell J. The General Practice Research Database. Scientific and Ethical Advisory Group. Ojm 1998;91:445-52.

15. Arts EE, Fransen J, den Broeder AA, et al. The effect of disease duration and disease activity on the risk of cardiovascular disease in rheumatoid arthritis patients. Ann Rheum Dis 2014.

16. de Groot V, Beckerman H, Lankhorst G], et al. How to measure comorbidity. a critical review of available methods. J Clin Epidemiol 2003;56:221-9.

17. Lewis JD, Brensinger C, Bilker WB, et al. Validity and completeness of the General Practice Research Database for studies of inflammatory bowel disease. Pharmacoepidemiol Drug Saf 2002;11:211-8.

18. Charlson ME, Pompei P, Ales KL, et al. A new method of classifying prognostic comorbidity in longitudinal studies: development and validation. Journal of chronic diseases 1987;40:373-83.

19. England BR, Sayles H, Mikuls TR, et al. Validation of the Rheumatic Disease Comorbidity Index. Arthritis Care Res (Hoboken) 2014.

20. Olomu AB, Corser WD, Stommel M, et al. Do self-report and medical record comorbidity data predict longitudinal functional capacity and quality of life health outcomes similarly? BMC Health Serv Res 2012; $12: 398$

21. Braun J, van den Berg R, Baraliakos X, et al. 2010 update of the ASAS/EULAR recommendations for the management of ankylosing spondylitis. Ann Rheum Dis 2011;70:896-904.

22. Wanders A, Heijde D, Landewe R, et al. Nonsteroidal antiinflammatory drugs reduce radiographic progression in patients with ankylosing spondylitis: a randomized clinical trial. Arthritis Rheum 2005;52:1756-65. 
23. Poddubnyy $\mathrm{D}$, Rudwaleit $\mathrm{M}$, Haibel $\mathrm{H}$, et al. Effect of non-steroidal anti-inflammatory drugs on radiographic spinal progression in patients with axial spondyloarthritis: results from the German Spondyloarthritis Inception Cohort. Ann Rheum Dis 2012;71:1616-22.

24. Kroon F, Landewe R, Dougados $M$, et al. Continuous NSAID use reverts the effects of inflammation on radiographic progression in patients with ankylosing spondylitis. Ann Rheum Dis 2012;71:1623-9.

25. Barnett K, Mercer SW, Norbury M, et al. Epidemiology of multimorbidity and implications for health care, research, and medical education: a cross-sectional study. Lancet 2012;380:37-43.

26. Van Spall HG, Toren A, Kiss A, et al. Eligibility criteria of randomized controlled trials published in highimpact general medical journals: a systematic sampling review. Jama 2007;297:1233-40.

27. Singal AG, Higgins PD, Waljee AK. A primer on effectiveness and efficacy trials. Clin Transl Gastroenterol 2014;5:e45.

28. Ozgocmen S, Khan MA. Current concept of spondyloarthritis: special emphasis on early referral and diagnosis. Curr Rheumatol Rep 2012;14:409-14.

29. Molto A, Paternotte S, Comet D, et al. Performances of the Assessment of SpondyloArthritis International Society axial spondyloarthritis criteria for diagnostic and classification purposes in patients visiting a rheumatologist because of chronic back pain: results from a multicenter, cross-sectional study. Arthritis Care Res (Hoboken) 2013;65:1472-81.

30. May C, Montori VM, Mair FS. We need minimally disruptive medicine. Brit Med ] 2009;339. 



\section{CHAPTER 10}

Summary

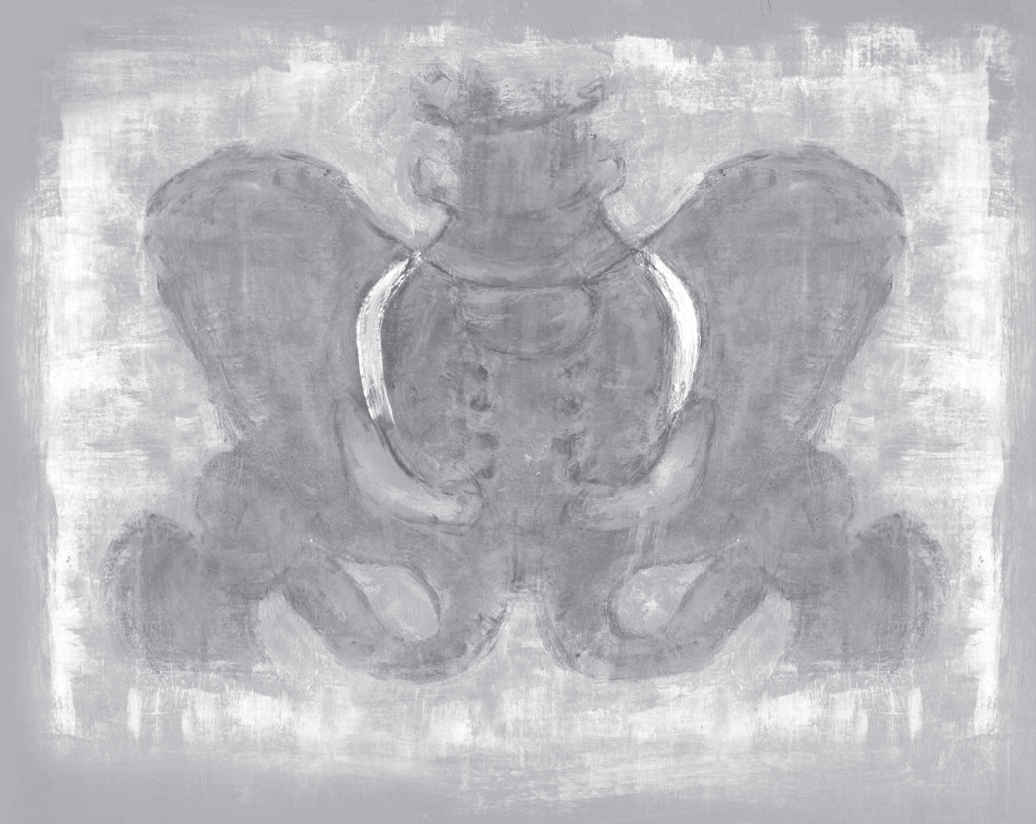





\section{SUMMARY}

Spondyloarthritis (SpA) is a group of interrelated chronic rheumatic diseases characterized by inflammation of the axial skeleton, inflammation of the peripheral joints, and extra-articular manifestations (EAMs) comprising acute anterior uveitis (AAU), psoriasis, and inflammatory bowel disease (IBD). The EAMs are considered to belong to the SpA concept. In addition to the articular and extra-articular SpA manifestations, patients may suffer from conditions which do not belong to the concept of SpA, such as cardiovascular disease. These conditions are not part of the SpA concept and are therefore referred to as comorbidities. Comorbidity is the general term for such conditions that occur in addition to an index disease.

This thesis focuses on the prevalence of SpA and aspects of conditions outside the joints in patients with SpA. Key questions addressed in this thesis include the epidemiology of SpA, the epidemiology of EAMs in patients with ankylosing spondylitis (AS), the risk of specific comorbidities, and the measurement of comorbidity for research purposes.

\section{Part I: Prevalence of Spondyloarthritis}

Chapter $\mathbf{2}$ describes a systematic literature review on the prevalence of SpA and its subtypes. In total, 84 studies were identified that estimated the prevalence of SpA, AS, psoriatic arthritis (PsA), reactive arthritis (ReA), SpA associated with $I B D$, and undifferentiated SpA (uSpA). For SpA, AS, and PsA, a sufficient number of studies was available to perform a meta-analysis. This meta-analysis showed a pooled population prevalence for SpA of $0.55 \%$ ( $95 \% \mathrm{Cl} 0.37-0.77$ ); for AS of $0.18 \%$ (95\% Cl 0.15-0.23); and for PsA of $0.15 \%$ (95\% Cl 0.12-0.18), with very high heterogeneity (>99\%) among the studies. Subgroupanalyses and meta-regression analyses were performed to identify demographical and methodological variables that could explain part of this heterogeneity. Geographic region was an important determinant of the prevalence of SpA and AS. Generally, it can be stated that prevalence estimates were higher in studies from Europe en North America, compared with Asia, the Middle East, and Africa. This may be particularly explained by differences in the prevalence of HLA-B27, which is associated with the prevalence of SpA. Further, the prevalence of SpA, and AS in particular, was higher in male compared with female subjects. The prevalence of PsA, on the other hand, was significantly higher in studies with a higher mean age of the population studied. With respect to methodological variables, the prevalences of SPA and PsA were positively related to the year of data collection (i.e. higher prevalences in more recent studies), whereas the prevalence of AS was not. Further, the case definition used was significantly related to the prevalence estimates. In general, prevalence estimates were higher in population studies in which a screening method followed by a confirmation phase was used compared with hospital or register based studies in which diagnosis was based on medical records or 
international classification of disease (ICD) codes. With respect the final confirmation of the case definition, prevalence estimates were higher when patients were classified according to the European Spondyloarthropathy Study Group (ESSG) criteria for SpA or the (modified) New York criteria for AS compared with other case definitions, such as medical records diagnoses.

\section{Part II: Epidemiology of extra-articular manifestations}

Chapter $\mathbf{3}$ and chapter $\mathbf{4}$ describe the epidemiology of EAMs in patients with AS, including the prevalence, the incidence, and the risks of developing an EAM in patients with AS compared with the general population. Chapter 3 comprises a systematic literature review on the prevalence of EAMs in patients with AS, and chapter 4 assesses the prevalence, incidence and risks of EAMs in patients with AS in the Clinical Practice Research Database (CPRD) from the United Kingdom. In the CPRD study, 4,101 patients with AS were matched with up to seven control subjects without AS by year of birth, sex, and practice $(n=28,591)$.

First, the epidemiology of acute anterior uveitis (AAU) in AS was studied. In the systematic review, a pooled prevalence of $25.8 \%$ ( $95 \% \mathrm{Cl} 21.1$ to 27.6 ) was found in 143 studies in which patients had a mean disease duration of AS of 15.9 (SD 5.9) years (chapter 3). In CPRD, a prevalence of AAU of $11.9 \%$ at diagnosis of AS and of $24.5 \%$ after 20 years of disease was found (chapter 4). In both studies, it was shown that the prevalence of AAU was significantly associated with disease duration of AS. In other words, patients with AS may develop a first episode of AAU many years after the diagnosis of AS. Further, it was shown in the review that the prevalence of AAU differs between geographic areas. The highest prevalence estimates of AAU in patients with AS were reported in studies from Europe and North America. Similarly to the prevalence of SpA itself, this could be explained first of all by differences in HLA-B27 in different geographic areas. With respect to the methodological factors, the prevalence of AAU was associated with selection of patients. The prevalence of AAU was on average lower in studies with a low risk of bias concerning random selection of patients. In CPRD, the incidence of AAU was 8.9 per 1000 person years in patients with AS, and 0.4 per 1000 person years in controls. After adjustment for possible confounders, the risk of developing a first episode of AAU after diagnosis of AS was 15.5-fold increased in patients compared with controls. This risk was highest in younger patients (16-39 years), in male patients and in patients with shorter disease duration. Nevertheless, 10 years after the index date, the risk of developing a first episode of AAU was still 9-fold increased.

Second, the epidemiology of psoriasis in AS was assessed. In the systematic review, a pooled prevalence of $9.3 \%$ (95\% Cl 8.1 to $10.6 \%$ ) was found, after a mean disease duration of AS of 16.7 (SD 6.2) years (chapter 3). No significant association with disease duration could be shown in the review. In CPRD, $4.1 \%$ of the patients were known with 
psoriasis at diagnosis of AS, and this proportion increased to $10.1 \%$ after 20 years (chapter 4). In the meta-regression analysis, the prevalence of psoriasis was significantly associated with geographic region. The highest prevalences were found in studies from Europe and Latin America. The incidence of psoriasis was 3.4 per 1000 person-years in patients with AS, and 1.8 per 1000 person-years in controls (chapter 4). Compared with population-based controls, the risk of psoriasis was 1.5-fold ( $95 \% \mathrm{Cl} 1.1-1.9)$ increased. The risk was only significantly increased in the first five years after diagnosis. Thereafter the risk was comparable with population based controls.

Third, the prevalence of IBD in AS was assessed. In the systematic review, the pooled prevalence of IBD was $6.8 \%(95 \% \mathrm{CI} 6.1$ to $7.7 \%)$ after a mean disease duration of 16.7 (SD 6.3) years (chapter 3 ). The prevalence of IBD found in the meta-analysis is in line with the CPRD-study, which found a prevalence of $4.0 \%$ at the index date of AS, and a prevalence of $7.5 \%$ of IBD after 20 years of AS (chapter 4 ). The prevalence of IBD in patients with AS was also associated with geographic region, with lower prevalences in studies from Asia compared with Europe. The incidence of IBD was 2.4 per 1000 personyears in patients with AS, and 0.4 per 1000 person-years in controls. Compared with the general population, the risk of developing IBD was 3-fold (95\% Cl 2.3-4.8) increased during follow-up, but was only significantly increased in the first ten years of follow-up. In chapter $\mathbf{5}$ the frequency of SpA features in patients with IBD was assessed. Three hundred and fifty consecutive patients with IBD who visited the outpatient clinic were questioned about the presence or history of possible SpA features including inflammatory back, peripheral arthritis, enthesitis, dactylitis, psoriasis and AAU. Medical records of all patients were checked to assess whether patients had ever visited a rheumatologist and whether they were diagnosed with any rheumatic diagnosis. Of all 350 patients, 129 (36.9\%) patients reported at least one musculoskeletal SpA feature: 79 (22.6\%) patients reported inflammatory back pain, 33 (9.4\%) reported peripheral arthritis, 47 $(12.0 \%)$ reported enthesitis, and 29 (8.3\%) patients reported dactylitis. Medical record review showed that $66(51.2 \%)$ patients had ever visited a rheumatologist. Axial SpA was diagnosed in 18 (27.3\%) of these patients, peripheral SpA in 20 (30.3\%) patients and another rheumatic disorder in $14(21.2 \%)$ patients. Strikingly, $49.8 \%$ of the patients with musculoskeletal complaints belonging to the SpA spectrum were never referred to a rheumatologist.

\section{Part III: Comorbidity in Ankylosing Spondylitis}

In chapter $\mathbf{6}$ we investigated the criterion and construct validity of the self-administered comorbidity questionnaire (SCO) in patients with AS. The SCO is a comorbidity questionnaire including 13 common medical conditions, developed to adjust for the impact of comorbidity on functional status. Ninety-eight patients who participated in the Outcome in AS International Study (OASIS) completed the SCO. In total, 64 (65.3\%) patients reported 
at least one non-rheumatic comorbidity. Criterion validity was assessed by the degree to which the self-reported comorbidities in the SCQ correlated with comorbidity data from the medical records. It was shown that patients can accurately report most comorbidities, except for stomach disease and depression, which were reported more frequently by patients than retrieved from the medical records. The agreement for rheumatic conditions included in the SCO was also low. Construct validity was assessed by correlating the SCO with other comorbidity scores: the Charlson index and the Michaud-Wolfe index (now called Rheumatic Disease Comorbidity Index, RDCI); and by correlating the SCO with demographics, physical function, health related quality of life (HROLL) and AS-related disease activity. These analyses were performed both for the 'original' SCQ as well as for a modified version of the SCO (mSCO), in which rheumatic conditions were removed, because these conditions are difficult to distinguish by patients from the index disease (i.e. AS). The correlations between the SCO and the Charlson index and Michaud-Wolfe index were low, but stronger for the mSCO. We further showed that the SCO correlated with age, HROoL and physical function, which adds to the construct validity of the SCO. In a multivariable regression analysis, the SCO and $\mathrm{mSCO}$ were significantly associated with HRQOL, physical function and work disability, the latter only in patients with low disease activity.

In chapter $\mathbf{7}$ the incidence and risks of cardiovascular morbidity was assessed in patients with AS compared with the general population, including the role of non-steroidalanti-inflammatory drugs (NSAIDs). All patients with newly diagnosed AS were identified from CPRD and matched with up to 7 controls. Hazard ratios (HR) for development of ischemic heart disease (IHD) and acute myocardial infarction (AMI) were calculated. Adjustments were made for age, gender, comorbidity, and drug use, including NSAIDs. The age-gender adjusted HR for developing IHD was not significantly increased (HR 1.20, 95\% $\mathrm{Cl}$ 0.97-1.48) in patients with AS. After stratification for gender, the risk of IHD was increased in female patients only (HR 1.88, 95\% Cl 1.22-2.90). After adjustment for potential confounders, the risk of IHD was not significantly increased in male (HR 0.94, 95\% Cl 0.73-1.21) nor in female patients (HR 1.31, 95\% Cl 0.86-2.08). Recent NSAID use explained this change for an important part, (HR IHD adjusted for age and NSAID in females $1.57,95 \% \mathrm{Cl}$ 0.99-2.48). In patients with AS who used an NSAID in the last three months before an event, the HR of IHD was significantly increased $(1.36,95 \% \mathrm{Cl} 1.00$ 1.85) compared with controls. The risk of developing IHD was particularly increased in patients who used a COX-2 inhibitor (HR 3.03, 95\% CI 1.61-5.69). The risk of developing an AMI was not significantly increased in patients with AS: the age-gender-adjusted HR was 0.91 (95\% Cl 0.65-1.28) and the fully-adjusted HR was 0.76 (95\% Cl 0.53-1.09).

Chapter 8 evaluated the effect of infliximab on symptoms of depression in patients with AS in a subgroup analysis of a randomized-controlled trial: the Ankylosing Spondylitis Study for the Evaluation of Recombinant Infliximab Therapy (ASSERT). Furthermore, this 
study tried to explore whether depressive symptoms in patients with AS are secondary to disease-related functional impairment and pain, or that depressive symptoms are the result of the inflammatory immune response, for example as a result of increased levels of anti-TNF-a. Patients were randomized to receive infliximab $(n=17)$ or placebo $(n=6)$ until week 24 after which all patients continued with infliximab until week 54. Depressive symptoms were measured with the Center for Epidemiological Studies Depression Scale (CES-D, range 0-60) at week $0,6,12,24$, and 54. We showed that the mean depression score was high at baseline (15.7, SD 8.0 ) and that $47.8 \%$ of patients had a CES-D score $\geq 16$, which is indicative of clinical depression. After six weeks of infliximab, the CES-D score had decreased in the infliximab group and was significantly lower in the infliximab group compared with the placebo group $(p=0.03)$. After 24 weeks, the mean CES-D score was still lower in the infliximab group than in the placebo group, although the difference did not reach statistical significance (CES-D score 10.8 (SD 11.4) versus 16.2 (SD 6.8), $p=0.07$ ). Generalized estimating equation (GEE) analyses of covariance showed a trend towards significance between the infliximab and placebo-group for CES-D scores over 24 weeks ( $p=0.06)$. At week $24,20 \%$ of the patients in the infliximab group and $57 \%$ of the infliximab group had a CES-D score indicating clinical depression $(p=0.17)$. In patients with depression at baseline in the infliximab group, the improvement in depression-scores was moderately related to the improvement in disease activity (BASDAI, $r=0.76, p=0.03$ ) and physical function (BASFI, $r=0.74, p=0.04$ ) at week 24. Importantly, the correlation between improvement in CES-D score and improvement in BASDAI score was lower after six weeks of treatment compared with later ascertainments, which may be explained by a faster improvement in depression scores than BASDAI scores. These findings suggest, but do not prove, that the improvement in depressive symptoms in patients with AS who were treated with infliximab were not only a result of improvement in pain and functional impairment, but also a result of a more direct effect of TNF-alpha inhibition.

In chapter $\mathbf{9}$ the main findings of this thesis were discussed. First, it was discussed how co-existing diseases should be conceptualized in patients with SpA. Pathogenetically, there is a clear difference between EAMs and comorbidities, because EAMs are disease manifestations belonging to the SpA concept rather than distinct entities, in contrast to comorbidities which lack this relation. For diagnosis and treatment in clinical practice and for outcome measurement in research, however, it often seems more practical to consider EAMs also as distinct entities. Second, methodological considerations were discussed including external validity and measurement of EAMs and comorbidity. Generalizability of the prevalence of SpA and EAMs is hampered by genetic differences in HLA-B27 among populations, whereas cause-relation studies are more generalizable, although effect-modification may play a role. Because comorbidity has an important impact on different outcomes, such as HROoL and participation, it is relevant to measure comorbidity. The approach of measuring comorbidity is dependent on the study type 
and research question. Third, implications for research and research challenges were discussed. This thesis contributed to the knowledge on EAMs and comorbidity in patients with SpA, but many more questions remain to be answered, for example with respect to the role of EAMs in the concept of SpA and etiopathogenic concepts of comorbidity. Finally, recommendations for clinical practice were made with respect to EAMs and comorbidity. The high prevalence of EAMs and comorbidity asks for a reorganization of the way we deliver healthcare. 


\section{CHAPTER 11}

\section{Samenvatting in het Nederlands}

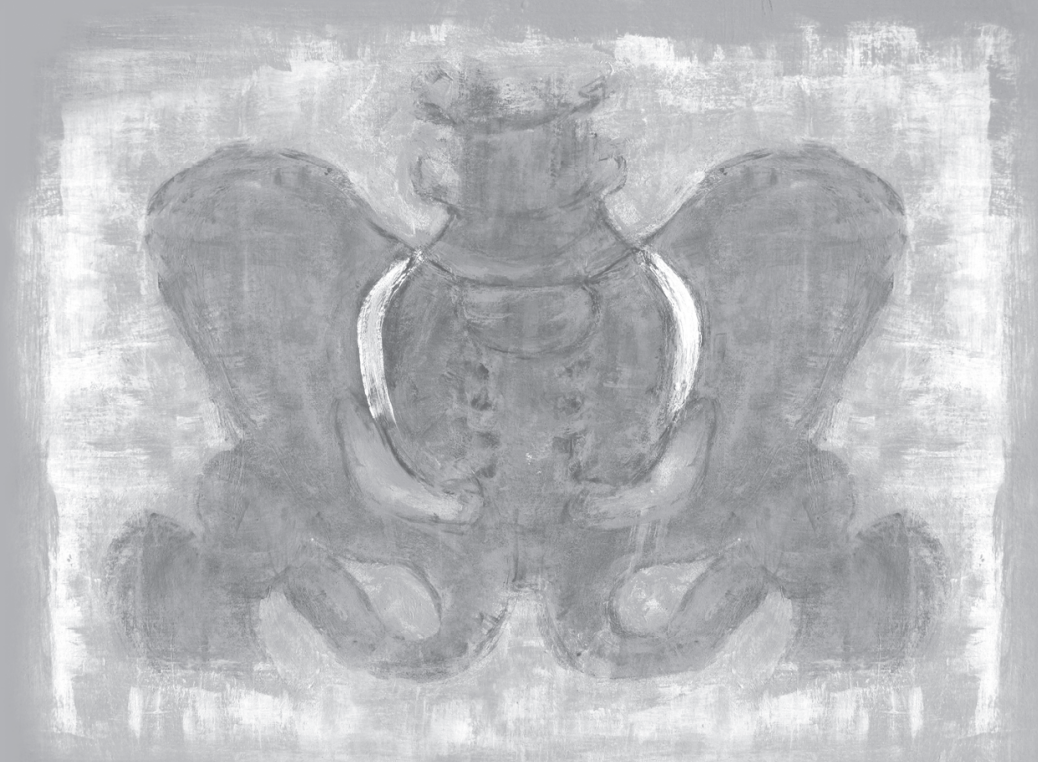





\section{SAMENVATTING}

Spondyloarthritis (SpA) is de verzamelnaam voor een groep van chronische reumatische aandoeningen. Deze aandoeningen worden gekenmerkt door ontsteking van de wervelkolom en de sacro-iliacale gewrichten (SI-gewrichten, ofwel heiligbeen gewrichten), wat leidt tot pijn en/of stijfheid van de (onder)rug en/of nek. Daarnaast komen er ontstekingen voor van de perifere gewrichten (in het bijzonder ontstekingen van de grote gewrichten zoals de knieën en de enkels) en de pezen, zoals de achillespees. Staan de symptomen van de rug op de voorgrond, dan spreken we van 'axiale SpA'. Staan de symptomen van de perifere gewrichten of pezen op de voorgrond, dan spreken we van 'perifere SpA'. SpA resulteert in veel beperkingen in het dagelijks leven en, mede doordat SpA meestal op jonge leeftijd begint (20-40 jaar), leidt de ziekte ook vaak tot werkverzuim of arbeidsongeschiktheid. Naast de gewrichtssymptomen manifesteert SpA zich ook vaak in andere organen, zoals in de ogen (acute uveitis anterior, AUA), de huid (psoriasis), en in de darmen (inflammatoire darmaandoeningen [IBD], zoals de ziekte van Crohn en colitis ulcerosa). Deze aandoeningen behoren tot het ziektebeeld SpA en worden ook wel extra-articulaire manifestaties (EAMs) genoemd. Patiënten met SpA kunnen natuurlijk ook andere ziektes ontwikkelen, zoals hart- en vaataandoeningen, osteoporose of een depressie. Sommige van deze aandoeningen komen vaker voor bij patiënten met SpA in vergelijking met de algemene populatie, bijvoorbeeld als een gevolg van de chronische ontsteking of als bijwerking van de medicatie die gebruikt wordt voor SpA. De algemene term voor het optreden van meer dan één chronische aandoening in één individu wordt 'multimorbiditeit' genoemd. Een andere term die vaak gebruikt wordt is 'comorbiditeit'. Dit betekent ook dat iemand meer dan één ziekte tegelijkertijd heeft, maar bij comorbiditeit wordt uit gegaan van een extra aandoening bij mensen die al een ziekte (de zogenaamde 'index-ziekte') hebben.

Het is bekend dat de aanwezigheid van twee of meer aandoeningen vaak leidt tot extra beperkingen in het fysiek functioneren, een verlies aan kwaliteit van leven, meer zorggebruik en hogere kans op sterfte. Het is daarom belangrijk om aandacht te hebben voor comorbiditeit en hier onderzoek naar te doen. In patiënten met SpA wordt dit onderzoek vaak gecompliceerd door het feit dat een deel van de aandoeningen die vaak voorkomen ook onderdeel zijn van SpA (zoals psoriasis of IBD). Daarnaast is het niet goed bekend hoe vaak EAMs en comorbiditeiten voorkomen bij patiënten met SpA en wat de achterliggende oorzaak is voor het vaker voorkomen van sommige aandoeningen. De onderzoeken zoals beschreven in dit proefschrift richten zich daarom op het voorkomen van EAMs en comorbiditeiten bij patiënten met SpA. De meeste onderzoeken hebben betrekking op patiënten met de ziekte van Bechterew, ook wel ankyloserende spondylitis (AS) genoemd. AS is de bekendste vorm van SpA. Kenmerkend voor AS is dat er sacroiliitis (ontsteking van het SI-gewricht) op een röntgenfoto gezien moet worden om de diagnose te stellen. 


\section{Deel I: Epidemiologie van spondyloarthritis}

Dit proefschrift begint in hoofdstuk 2 met een systematisch literatuuronderzoek naar de prevalentie van SpA en de subtypen van SpA. In totaal werden er 84 artikelen geïdentificeerd warin de prevalentie van SpA, AS, artritis psoriatica (PsA), reactieve artritis (ReA), SpA geassocieerd met IBD en ongedifferentieerde SpA werd gerapporteerd. Voor SpA, AS en PsA waren er voldoende artikelen beschikbaar om een meta-analyse uit te voeren. Deze meta-analyse toonde een gepoolde populatie prevalentie van SpA van $0.55 \%$ (95\% betrouwbaarheidsinterval (BI) 0.37-0.77); van AS van 0.18\% (95\% BI 0.15-0.23); en van PsA van $0.15 \%$ (95\% BI 0.12-0.18), met hoge heterogeniteit (>99\%) tussen de studies. Subgroep analyse en meta-regressie analyse werden uitgevoerd om klinische en methodologische variabelen te identificeren die een deel van deze heterogeniteit konden verklaren. De geografische regio was een belangrijke determinant voor de prevalentie van SpA en AS. Over het algemeen kan gezegd worden dat de prevalentie hoger is in studies uit Europa en Noord-Amerika, in vergelijking met Azië, het Midden-Oosten en Afrika. Dit kan voornamelijk verklaard worden door verschillen in de prevalentie van HLAB27 tussen de regio's. Daarnaast is de prevalentie van SpA en AS hoger in mannen dan in vrouwen. De prevalentie van PsA is hoger in studies waarin de gemiddelde leeftijd van de onderzochte populatie hoger ligt. Aangaande de methodologische variabelen was de prevalentie van SpA en PsA positief gerelateerd aan het jaar van data collectie. Daarnaast was de manier waarop de patiënten werden gedefinieerd en geclassificeerd significant gerelateerd aan de prevalentie. Prevalenties waren hoger in populatiestudies waarin een screeningsmethode werd gebruikt gevolgd door een confirmatiefase in vergelijking met ziekenhuis- of database-studies waarin de patiënten werden geïdentificeerd vanuit medische dossiers of op basis van ICD-codes. Bovendien waren de prevalentiecijfers hoger wanneer patiënten werden geclassificeerd op basis van de 'European Spondyloarthropathy Study Group' (ESSG) criteria voor SpA of de (gemodificeerde) New York criteria voor AS in vergelijking met andere definities, zoals medische dossiers.

\section{Deel II: Epidemiologie van extra-articulaire manifestaties}

Hoofdstuk $\mathbf{3}$ en hoofdstuk $\mathbf{4}$ beschrijven de epidemiologie van EAMs in patiënten met AS. Er werd in deze studies gekeken naar de prevalentie, de incidentie en het risico om een EAM te ontwikkelen in AS patiënten in vergelijking met de algemene populatie. Hoofdstuk 3 bevat een systematisch literatuur onderzoek naar de prevalentie van EAMs in patiënten met AS en hoofdstuk 4 onderzoekt de prevalentie, incidentie en risico's op het ontwikkelen van EAMs in de 'Clinical Practice Research Database' (CPRD) uit het Verenigd Koninkrijk. In deze laatste studie werden 4.101 patiënten met AS geïdentificeerd in de database en gekoppeld aan 28.591 controlepatiënten zonder AS op basis van geboortejaar, geslacht en huisartspraktijk. 
Ten eerste werd de epidemiologie van AUA bestudeerd. In het systematische literatuur onderzoek werd een gepoolde prevalentie van 25.8\% (95\% BI 21.1-27.6) gevonden in 143 studies waarin patiënten een gemiddelde ziekteduur van AS van 15.9 (standaard deviatie (SD) 5.9) jaar hadden. In CPRD was de prevalentie van AUA op het moment van diagnose van AS $11.9 \%$ en na 20 jaar $24.5 \%$. In beide studies werd gevonden dat de prevalentie van AUA geassocieerd was met de ziekteduur van AS. Met andere woorden, patienten met AS kunnen jaren na de diagnosestelling van AS is nog een eerste episode van AUA ontwikkelen. Verder werd aangetoond dat de prevalentie van AUA verschillend was tussen geografische regio's. De hoogste prevalentie van AUA in patiënten met AS werd gerapporteerd in studies uit Europa en Noord-Amerika. Dit kon deels verklaard worden door verschillen in prevalentie van HLA-B27 tussen verschillende regio's. Met betrekking tot methodologische factoren was de prevalentie van AUA geassocieerd met selectie van patiënten. De prevalentie van AUA was gemiddeld lager in studies met een laag risico op bias door random selectie van patiënten. De incidentie van AUA in patiënten met AS in CPRD was 8.9 per 1.000 persoonsjaren en 0.4 per 1.000 persoonsjaren in controles. Na correctie voor potentiële confounders, was het risico op het ontwikkelen van een eerste episode van AUA 15.5-maal verhoogd in patiënten met AS vergeleken met controles. Dit risico was hoger in jonge patiënten (16-39 jaar), in mannen en in patiënten met een kortere ziekteduur (< 1 jaar). Niettemin was het risico 10 jaar na de diagnose van AS nog steeds 9-maal verhoogd.

Ten tweede werd de epidemiologie van psoriasis in patiënten met AS onderzocht. In de systematische literatuur studie werd een gepoolde prevalentie van 9.3\% (95\% BI 8.1-10.6\%) gevonden in patiënten met een gemiddelde ziekteduur van AS van 16.7 (SD 6.2) jaar. Er kon geen significante associatie met ziekteduur worden aangetoond. In CPRD was $4.1 \%$ van de patiënten bekend met psoriasis ten tijde van de diagnose AS, en dit percentage nam toe tot $10.1 \%$ na 20 jaar. In de meta-regressie analyse was de prevalentie van psoriasis in patiënten met AS geassocieerd met geografische regio. De hoogste prevalenties werden gevonden in studies uit Europa en Zuid-Amerika. De incidentie van psoriasis was 3.4 per 1.000 persoonsjaren in AS patiënten en 1.8 per 1.000 persoonsjaren in controles. In vergelijking met controles uit de algemene populatie was het risico op het ontwikkelen van psoriasis 1.5-maal (95\% Cl 1.1-1.9) verhoogd in patiënten met AS. Echter, het risico was alleen significant verhoogd in de eerste vijf jaar na diagnose. Daarna was het risico vergelijkbaar met dat in de algemene populatie.

Ten slotte werd de prevalentie van IBD in patiënten met AS onderzocht. De gepoolde prevalentie van IBD was $6.8 \%$ (95\% BI 6.1-7.7\%) in de meta-analyse in patiënten met een gemiddelde ziekteduur van AS van 16.7 (SD 6.3) jaar. De prevalentie was significant verschillend tussen geografische regio's met lagere prevalenties in studies in Azië vergeleken met Europa. De IBD prevalentie die in de meta-analyse werd gevonden is in overeenstemming met de CPRD studie warin een prevalentie van $4.0 \%$ ten tijde 
van de diagnose van AS en van 7.5\% na 20 jaar werd gevonden. De incidentie van IBD was 2.4 per 1.000 persoonsjaren in patiënten met AS en 0.4 per 1.000 persoonsjaren in controles. In vergelijking met de algemene populatie was het risico op IBD 3-maal (95\% BI 2.3-4.8) verhoogd in patiënten met AS. Het risico was significant hoger in de eerste 10 jaar na de diagnose AS.

In hoofdstuk 5 werd de prevalentie van SpA symptomen in patiënten met IBD onderzocht. Er werden 350 patiënten geïnterviewd die opeenvolgend de polikliniek van de maag-darm-leverziekten bezochten over de aanwezigheid of een voorgeschiedenis van inflammatoire rugpijn, perifere artritis, enthesitis, dactylitis, psoriasis en AUA. Medische dossiers van alle patiënten werden systematisch bekeken om na te gaan of deze patiënten ooit een reumatoloog bezochten en of zij gediagnosticeerd waren met een reumatische aandoening. Van alle 350 patiënten rapporteerden er 129 (36.9\%) ten minste één SpA symptoom gerelateerd aan het bewegingsapparaat: 79 (12.0\%) patiënten rapporteerde inflammatoire rugpijn; 33 (9.4\%) patiënten rapporteerden perifere artritis; 47 (12.0\%) patiënten rapporteerden enthesitis; en 29 (8.3\%) patiënten rapporteerden dactylitis. In de medische dossiers werd gevonden dat 66 (51.2\%) van deze patiënten ooit een reumatoloog hadden bezocht. Axiale SpA was gediagnosticeerd in 18 (27.3\%) van de 66 patiënten, perifere SpA in 20 (30.3\%) patiënten, en een andere reumatische aandoening in 14 (21.2\%) patiënten. Opvallend was dat $49.8 \%$ van de patiënten die ten minste één symptoom van het bewegingsapparaat welke bij SpA zouden kunnen passen rapporteerde, nooit verwezen was naar een reumatoloog.

\section{Deel III: Comorbiditeit in ankyloserende spondylitis}

In hoofdstuk 6 werd de criterium- en de constructvaliditeit van de 'self-administered comorbidity questionnaire' (SCO) onderzocht in patiënten met AS. De SCQ is een comorbiditeits-vragenlijst die vragen bevat over 13 medische aandoeningen. De SCO is ontwikkeld om functionele status te corrigeren voor de invloed van comorbiditeit. Achtennegentig patiënten die meededen aan de 'Outcome in AS International Study' (OASIS) vulden de SCO in. In totaal rapporteerden 64 patiënten ten minste één nietreumatische comorbiditeit. Criteriumvaliditeit werd bepaald aan de aan hand van de mate waarin de zelf-gerapporteerde comorbiditeiten overeenkwamen met gegevens over comorbiditeiten in de medische dossiers. Er werd aangetoond dat patiënten de meeste comorbiditeiten accuraat kunnen rapporteren. Echter, maagaandoeningen en depressie werden vaker gerapporteerd door patiënten dan werd gevonden in de medische dossiers. Verder was de overeenstemming voor reumatische aandoeningen die in de SCQ staan laag. Constructvaliditeit werd bepaald door de SCO te correleren met andere comorbiditeitsvragenlijsten: de Charlson-index en de Michaud-Wolfe index. Daarnaast werd de correlatie van de SCO berekend met demografische kenmerken, fysiek functioneren, gezondheids-gerelateerde kwaliteit van leven (KvL) en AS-gerelateerde ziekte 
activiteit. Deze analyses werden uitgevoerd zowel voor de SCQ als voor een gemodificeerde versie van de SCO (mSCO), waarin de reumatische items waren verwijderd omdat deze voor patiënten moeilijk te onderscheiden zijn van de index ziekte (d.w.z. AS). De correlaties tussen de SCQ en zowel de Charlson index als de Michaud-Wolfe waren zwak, maar sterker voor de mSCQ en deze comorbiditeitsvragenlijsten. De SCQ correleerde significant met leeftijd, KvL en fysiek functioneren, wat pleit voor constructvaliditeit. In een multivariabele regressie analyse waren de SCO en de mSCO significant geassocieerd met $\mathrm{KvL}$, fysiek functioneren en arbeidsongeschiktheid, de laatste alleen in patiënten met lage ziekteactiviteit.

In hoofdstuk $\mathbf{7}$ werd de incidentie en het risico op cardiovasculaire morbiditeit bepaald in patiënten met AS in vergelijking met de algemene populatie, met speciale aandacht voor de rol van non-steroïdale anti-inflammatoire geneesmiddelen (NSAID's) op dit risico. Alle patiënten die nieuw gediagnosticeerd waren met AS werden geïdentificeerd in de CPRD database en gekoppeld aan 7 controlepersonen zonder AS. Hazard ratio's (HR) voor de ontwikkeling van ischemische hartaandoeningen (IHD) en acuut myocardinfarct (AMI) werden berekend. Er werden correcties gemaakt voor leeftijd, geslacht, comorbiditeit en medicijngebruik, waaronder NSAID's. Het voor leeftijd-geslacht gecorrigeerde risico op het ontwikkelen van IHD was niet significant verhoogd in patiënten met AS vergeleken met controles zonder AS (HR 1.20,95\% BI 0.97-1.48). Na stratificatie voor geslacht was het risico wel significant verhoogd in vrouwelijke AS patiënten (HR 1.88, 95\% BI 1.222.90). Na correctie voor alle potentiële confounders was het risico op het ontwikkelen van IHD zowel in mannen (HR 0.94, 95\% BI 0.73-1.21) als in vrouwen (HR 1.31, 95\% BI 0.86-2.08) niet significant verhoogd. Voor een belangrijk deel kon deze verschuiving in HR verklaard worden door NSAID gebruik (HR IHD in vrouwen gecorrigeerd voor leeftijd en NSAID gebruik: 1.57, 95\% BI 0.99-2.48). In patiënten met AS, die in de laatste drie maanden een NSAID hadden gebruikt, was het risico op het ontwikkelen van IHD significant verhoogd $(1.36,95 \% \mathrm{BI} 1.00-1.85)$ in vergelijking met controles. Het risico voor het ontwikkelen van IHD was vooral verhoogd in patiënten die een COX-2 inhibitor gebruikten (HR 3.03, 95\% Cl 1.61-5.69). Het risico op het ontwikkelen van een AMI was niet significant verhoogd in patiënten met AS. De voor leeftijd-geslacht gecorrigeerde HR voor het ontwikkelen van een AMI was 0.91 (95\% BI 0.65-1.28) en na correctie voor alle potentiele confounders was de HR 0.76 (95\% BI 0.53-1.09).

In hoofdstuk 8 werd het effect van infliximab op symptomen van depressie in AS patiënten onderzocht in een subgroep analyse van een gerandomiseerde trial: the Ankylosing Spondylitis Study for the Evaluation of Recombinant Infliximab Therapy (ASSERT). Daarnaast probeerde deze studie te exploreren of depressieve symptomen in patiënten met AS secundair zijn aan ziektegerelateerde functionele beperkingen en pijn, of dat depressieve symptomen het resultaat zijn van de inflammatoire immuunrespons, bijvoorbeeld als gevolg van verhoogde TNF-alfa spiegels. Patiënten werden gerandomiseerd om 
infliximab toegediend te krijgen $(n=17)$ of placebo $(n=6)$ tot week 24, waarna alle patiënten overgingen op infliximab tot week 54. Depressieve symptomen werden gemeten met de 'Center for Epidemiological Studies Depression Scale' (CES-D, range 0-60) in week 0, 6, 12, 24 en 54. Op baseline was de gemiddelde depressiescore hoog (CES-D score 15.7, SD 8.0) en had $47.8 \%$ van de patiënten een CES-D score $\geq 16$, wat suggestief is voor een klinische depressie en een indicatie is voor nader onderzoek. Na 6 weken behandeling met infliximab was de CES-D score afgenomen en was deze significant lager dan in de placebogroep $(p=0.03)$. Na 24 weken was de CES-D score nog steeds lager in de infliximabgroep vergeleken met de placebogroep, hoewel het verschil net niet statistisch significant was (CES-D score 10.8 (SD 11.4) versus 16.2 (SD 6.8), p=0.07). Generalized estimating equation (GEE) analyses of covariance toonde een trend voor significantie voor een verschil tussen de infliximabgroep en de placebogroep over 24 weken $(p=0.06)$. In week 24 had $20 \%$ van de patiënten in de infliximabgroep en $57 \%$ van de patiënten in de placebogroep een CES-D score die richtinggevend was voor een klinische depressie $(p=0.17)$. In patiënten met een CES-D score $\geq 16$ op baseline die in de infliximabgroep zaten, was de verbetering in CES-D score redelijk goed gecorreleerd met de verandering in ziekteactiviteit (BASDAl, $r=0.76, p=0.03$ ) en fysiek functioneren (BASFI, $r=0.74, p=0.04$ ) score na 24 weken. Echter, deze correlatie was lager na de eerste 6 weken, wat mogelijk verklaard kan worden door een snellere verbetering van depressiescores dan de BASDAI scores. Deze bevindingen suggereren (maar bewijzen dit niet) dat de verbetering in depressieve symptomen in patiënten met AS, die behandeld worden met infliximab, niet alleen het resultaat is van verbetering in pijn en fysiek functioneren, maar ook een direct resultaat van TNF-alfa remming.

In hoofdstuk 9 werden de belangrijkste bevindingen uit dit proefschrift bediscussieerd. Ten eerste werd er besproken hoe bijkomende ziektes in patiënten met SpA geconceptualiseerd moeten worden. Pathogenetisch gezien is er een duidelijk verschil tussen EAMs en comorbiditeiten, omdat EAMs, in tegenstelling tot comorbiditeiten, ziektemanifestaties zijn die tot het concept van SpA behoren en geen losstaande aandoeningen. Voor de diagnose en behandeling in de dagelijkse praktijk en voor het meten van uitkomsten in onderzoek, is het echter mogelijk geschikter om EAMs ook als aparte entiteiten te beschouwen. Ten tweede werden er enkele methodologische overwegingen besproken met betrekking tot de externe validiteit en het meten van EAMs en comorbiditeiten. De generaliseerbaarheid van de prevalentie van SpA en EAMs wordt vooral beperkt door genetische verschillen, zoals bijvoorbeeld de aanwezigheid van HLA-B27, tussen populaties. Oorzaak-gevolg relaties zijn beter te generaliseren, hoewel effect-modificatie een belangrijke invloed kan hebben. De manier waarop comorbiditeiten het beste gemeten kunnen worden is ook vooral afhankelijk van het studietype en de onderzoeksvraag. Ten derde werden er implicaties en uitdagingen voor toekomstig onderzoek besproken. Deze 
thesis heeft een relevante bijdrage geleverd aan de kennis over EAMs en comorbiditeiten in patiënten met SpA, maar er blijven nog veel vragen die nog beantwoord moeten worden, zoals met betrekking op de rol van EAMs in het concept SpA en etiopathogenetische concepten van comorbiditeit. Ten slotte werden er aanbevelingen gedaan voor de klinische praktijk met betrekking tot EAMs en comorbiditeiten. De hoge prevalentie van EAMs en comorbiditeiten in SpA vraagt voor een aanpassing van de manier waarop de zorg momenteel geleverd wordt. 



\section{VALORISATION ADDENDUM}

Valorization is the process of "translating academic wisdom to societal benefit". In this thesis we focused on the epidemiology and measurement of extra-articular manifestations (EAMs) and comorbidities in patients with spondyloarthritis (SpA) and ankylosing spondylitis (AS). This addendum describes the societal relevance of the present findings.

\section{Prevalence of SpondyloArthritis}

Data on the prevalence of SpA were limited available, but increasingly important. In the last decade, more treatments have become available for $\mathrm{SpA}$; in particular the introduction of anti-TNF-alpha therapy dramatically changed the outcome of patients with SpA. However, anti-TNF-alpha therapy is expensive and may have large impact on health budgets when frequently prescribed. In this thesis we have shown that SpA and its subtypes are a relatively common disease. The pooled prevalence of SpA was $0.54 \%$ (0.36-0.78) and that of AS 0.25\% (0.18-0.33) in Europe. These numbers can be used in budget impact analyses to estimate the financial consequences for society for example when new biologicals or biosimilars will become available in the near future.

\section{Extra-articular manifestations in Ankylosing Spondylitis}

AS is the prototype of the SpA group and is characterized by inflammation of the sacroiliac joints and the vertebrae, causing pain and stiffness in the back and/or buttock area. Patients with AS may also suffer from other manifestations belonging to the SpA concept, such as peripheral arthritis, enthesitis, or EAMs comprising acute anterior uveitis (AAU), psoriasis, and inflammatory bowel disease (IBD). All these clinical symptoms and subsequent disease progression result in substantial functional limitations and lower quality of life.

Symptoms associated with AS usually start in the $2^{\text {nd }}$ or $3^{\text {rd }}$ decade of life when development of a personal career and raising a family life are important social roles. In several studies it has been shown that patients with AS have lower employment rates, incur more official work disability, and experience more absences from work than the general population $[1,2]$. The clinical burden of disease leads to significant direct and indirect costs for patients and society [3]. In order to decrease both the burden of disease for patients and costs for society, appropriate and early treatment is needed. Early treatment is particularly important, since several observations suggests that early effective treatment may influence radiographic outcome [4].

Importantly, in order to provide early treatment, patients should be diagnosed early and patients with poor prognosis should be identified. The tools available for rheumatologists to diagnose patients with axial SpA (axSpA) have improved substantially in the last years, for example with the introduction of MRI to detect sacroiliitis. However, the diagnosis of 
AS is often delayed as a result of late recognition of patients. This may be caused by the insidious onset of symptoms, the heterogeneous picture, and the limited knowledge on manifestations belonging to the concept of SpA by general practitioners (GPs) and other referring physicians. Many patients present to GPs with back pain and not all patients with axSpA suffer from the typical inflammatory back pain. This makes it difficult for GPs to refer the right patients to rheumatologists.

One of the fundamental aspects to improve early recognition of SpA patients is knowledge and recognition of disease patterns by GPs and other physicians [5]. These patterns include axial symptoms, peripheral symptoms and EAMs. In this thesis, we showed that EAMs are frequently present before the diagnosis of AS. More than $11 \%$ of patients had an episode of AAU, more than $4 \%$ had psoriasis, and almost $4 \%$ had IBD before AS was diagnosed. These findings prove the relevance of EAMs in the early phase of SpA. The EAMs may particularly help to recognize patients who present with chronic back pain and possibly have SpA. Actively asking about other SpA features in these patients is needed. Further, in this thesis we showed that half of the patients with IBD who reported articular SpA features were never referred to a rheumatologist by their gastroenterologist. Education and increasing awareness of GPs and other specialists who see patients with a possible SpA about features belonging to SpA, including EAMs, is therefore warranted. It is also important to realize that half of the patients develop an EAM after the diagnosis of AS. This is particularly important for AAU, because AAU needs immediate treatment by an ophthalmologist to prevent possible visual impairment. It is important to educate patients about the symptoms of AAU and about the fact that a first episode of AAU can also present some decades after the diagnosis of AS.

The presence of EAMs are also important in light of the choice of treatment. Most TNFblocking agents are effective both for SpA and refractory uveitis, psoriasis, and/or IBD, although differences may exist among the available agents [6]. Since anti-TNF medication is expensive and economic evaluations showed substantial direct and indirect costs associated with AAU and IBD flares in patients with AS, it is important to take EAMs into account when selecting the most appropriate treatment.

\section{Multimorbidity}

In addition to EAMs, many patients with AS suffer from other chronic diseases, which may or may not be related to AS. The World Health Organization (WHO) defines chronic diseases as diseases of long duration and generally slow progression [7]. According to the $\mathrm{WHO}$, chronic diseases are the leading cause of mortality and morbidity in Europe. The prevalence of chronic diseases and also of combinations of chronic disease rises. Clinical population studies showed that multimorbidity is common and in the Dutch general population, $29.7 \%$ suffer from multimorbidity [8]. A systematic literature review showed a prevalence of $60 \%$ of multimorbidity among people aged 55 to 74 [9]. Although its 
prevalence increases with age, it is not a problem limited to the elderly population. Multimorbidity is associated with high mortality, reduced functional status, and increased use of both inpatient and ambulatory health care [10]. In the USA, approximately $80 \%$ of Medicare spending is devoted to patients with 4 or more chronic diseases, with costs exponentially increasing with higher multimorbidity [11]. In this thesis, we showed that many patients with AS suffer from multimorbidity and that having more than one disease in addition to AS resulted in lower quality of life, impaired function and more work disability.

The findings in the literature and of the present thesis have several implications for society and organization of care. Traditionally, health care and treatment strategies mainly focused on single-diseases without considering the broader context of multiple risk factors and co-occurring chronic conditions. Most evidence based medicine guidelines are not developed for patients with multimorbidity and do not consider related consequences such as polypharmacy. Strategies to manage different chronic diseases create a growing burden for patients [12]. Different clinicians offer care, which may lead to uncoordinated prescriptions and polypharmacy, increasing treatment costs, side effects, and unintended drug interactions. To optimize care of patients with multimorbidity, health care services are needed that are coordinated at the patient level. In patients with SpA, for example, multidisciplinary consultation hours in which rheumatologists, dermatologists, gastroenterologists, and ophthalmologists are working together may improve care and decrease the burden of treatment for patients.

In light of prevention, it is important to learn more about which diseases are more prevalence or which patients are at risk to develop specific comorbidities. In this thesis we investigated the risk of cardiovascular disease in patients with AS. Cardiovascular disease is one of the leading causes of death and loss of quality of life worldwide. Understanding the underlying association between AS and cardiovascular disease may help determine the targets of prevention. The cardiovascular risk is now well established in patients with rheumatoid arthritis. In this thesis it was shown that the increased risk of cardiovascular disease was only higher in women with AS, but this was mainly explained by NSAID use in this group. In particular, the risk of ischemic heart disease was increased in patients who used a COX-2 inhibitor. The risk of acute myocardial infarction, however, was not increased in patients with AS. Therefore, it seems that there is no need to include patients with AS in large cardiovascular prevention programs, as is the case with rheumatoid arthritis. There is probably more potential in carefully selecting the appropriate class of NSAIDs in the lowest possible dose.

Mental health problems are often an underestimated comorbidity in patients with chronic diseases, such as in patients with AS. These conditions may have large impact both on patients and society. It has been shown that comorbid depression is often associated with a more severe course of the physical disorder, partly because of non-adherence to 
treatment regimens in depressed patients [13]. Further, it has been shown that improvement in depression outcome was associated with decreased somatic symptoms without improvement in physiologic measures [14]. In AS, only limited information is available about the prevalence and treatment of depressive symptoms. In this thesis, we showed in a subgroup analysis of a randomized controlled trial that depressive symptoms were commonly present and improved with anti-TNF-alpha therapy. This study was a first step in the research on the effects of treatment of depression among people with AS and further larger studies are warranted.

In summary, SpA and AS as a subgroup, are common diseases with a significant burden on patients and society. Because AS usually starts at a young age and AS may have large impact on functioning, the socioeconomic impact of the disease can be high. With the introduction of anti-TNF-alpha treatment, effective treatment has now become available for SpA. Anti-TNF-alpha treatment, however, is expensive and reliable estimates of the prevalence of SpA and AS are useful for health care budgets. Since remission rates are highest in the early stages of disease, early recognition of SpA is important. In particular improving knowledge of GPs and other physicians about disease patterns of SpA and including EAMs will help to achieve this. As many patients suffer from EAMs or comorbidities, reorganization of health care delivery for these patients is important to improve quality of care. 


\section{REFERENCES}

1. Boonen A, Chorus A, Miedema H, et al. Employment, work disability, and work days lost in patients with ankylosing spondylitis: a cross sectional study of Dutch patients. Ann Rheum Dis 2001;60:353-8.

2. Mau W, Listing J, Huscher D, et al. Employment across chronic inflammatory rheumatic diseases and comparison with the general population. ] Rheumatol 2005;32:721-8.

3. Boonen A, van der Linden SM. The burden of ankylosing spondylitis. J Rheumatol Suppl 2006;78:4-11.

4. Robinson PC, Brown MA. The window of opportunity: a relevant concept for axial spondyloarthritis. Arthritis Res Ther 2014;16:109.

5. van Onna M, Gorter S, van Meerendonk A, et al. General practitioners' perceptions of their ability to identify and refer patients with suspected axial spondyloarthritis: a qualitative study. J Rheumatol 2014; 41:897-901.

6. Gao X, Wendling D, Botteman MF, et al. Clinical and economic burden of extra-articular manifestations in ankylosing spondylitis patients treated with anti-tumor necrosis factor agents. ] Med Econ 2012;15: 1054-63.

7. Organization WH. Chronic diseases. Available from: http://who.int/topics/chronic_disease/en/.

8. van den Akker M, Buntinx F, Metsemakers JF, et al. Multimorbidity in general practice: prevalence, incidence, and determinants of co-occurring chronic and recurrent diseases. J Clin Epidemiol 1998;51:367-75.

9. Fortin M, Lapointe L, Hudon C, et al. Multimorbidity is common to family practice: is it commonly researched? Can Fam Physician 2005;51:244-5.

10. Fortin M, Soubhi H, Hudon C, et al. Multimorbidity's many challenges. Bmj 2007;334:1016-7.

11. Wolff JL, Starfield B, Anderson G. Prevalence, expenditures, and complications of multiple chronic conditions in the elderly. Arch Intern Med 2002;162:2269-76.

12. May C, Montori VM, Mair FS. We need minimally disruptive medicine. Brit Med ] 2009;339.

13. Kessler RC. The costs of depression. Psychiatr Clin North Am 2012;35:1-14.

14. Katon W, Lin EH, Kroenke K. The association of depression and anxiety with medical symptom burden in patients with chronic medical illness. Gen Hosp Psychiatry 2007:29:147-55. 



\section{DANKWOORD}

Ruim drie jaar later is het dan zo ver, het boekje is af! Het was een mooie en leerzame periode, mede door de hulp van vele mensen om mij heen. Een aantal mensen wil ik hiervoor in het bijzonder bedanken.

Professor Boonen, beste Annelies, het was ontzettend fijn om jou als promotor te hebben. Ondanks dat je het altijd druk hebt maakte je altijd tijd voor me vrij. Door jouw enthousiasme, nieuwe ideeën en het vertrouwen dat je in mij had, heb ik zelf ook altijd vertrouwen gehad in een goede afloop. Het was altijd inspirerend om met je te overleggen en ik heb er bewondering voor hoe je zoveel ballen hoog houdt. Bedankt voor alles wat ik van je geleerd heb en je enorme betrokkenheid!

Dr. Van Tubergen, beste Astrid, jij was degene die mij tijdens mijn WESP stage bij jullie op de afdeling op het idee bracht om promotieonderzoek te gaan doen. Ik heb hier geen moment spijt van gehad! Ik heb heel erg veel geleerd van je wetenschappelijke adviezen en kritische blik. Artikelen kwamen vooral in het begin soms helemaal rood terug, maar altijd met een opmerking erbij dat 'dit heel normaal was' :- Ik wil je ontzettend bedanken voor je betrokkenheid, begeleiding, vertrouwen en de kansen die je mij geboden hebt.

Ik wil graag de leden van de beoordelingscommissie, prof. dr. N. Schaper, prof. dr. M.H. Prins, prof. dr. M. Boers, dr. F. van Gaalen en Dr. M. van den Akker, bedanken voor de tijd die jullie hebben genomen om mijn proefschrift te lezen en te beoordelen.

Dan natuurlijk ook een speciaal woord van dank voor alle coauteurs die mee hebben gewerkt aan de verschillende hoofdstukken van dit proefschrift. Sofia, oneindig veel röntgenfoto's hebben we gescoord en oneindig veel korte en lange mensen hebben we gemeten op de lange-mensen dag, de NVR en in het ziekenhuis; het was altijd gezellig. Ik heb ook op wetenschappelijk gebied veel van je geleerd en weet zeker dat jij het ver gaat schoppen, bedankt!

Prof. Landewé en prof. van der Heijde, beste Robert en Désirée, bedankt voor jullie kritische commentaar en feedback op de artikelen waaraan we samen hebben gewerkt. Prof. Dougados en prof van den Bosch, thank you for your comments and suggestions to the OASIS papers.

Dr. de Vries en dr. Bazelier, beste Frank en Marloes, ik wil jullie bedanken voor de prettige samenwerking aan de GPRD studies. Marloes, bedankt voor je geduld en tijd om Ivette en mij een beetje weg wijs te maken in SAS. Frank, ik heb veel geleerd van je adviezen om efficiënt een artikel te schrijven en zal deze zeker nog vaker gaan gebruiken. Prof. Masclee en dr. Pierik, om onderzoek te doen naar comorbiditeiten is samenwerking tus- 
sen verschillende specialismen noodzakelijk. Jullie waren betrokken bij mijn allereerste artikel en daarvoor wil ik jullie bedanken.

Lieve mede-reumatologie-promovendi, mede door jullie had ik elke dag weer zin om te gaan werken en ik heb ook erg genoten van alle activiteiten die we buiten het werk hebben ondernomen! We hebben heel veel leuke en minder leuke momenten met elkaar gedeeld en het was fijn om zulke betrokken collega's te hebben.

Ivette, wat was het leuk jou te leren kennen, zowel als collega maar ook op persoonlijk vlak. We hebben een aantal artikelen samen geschreven en het was fijn met jou samen te werken. Maar ik heb ook erg veel goede herinneringen aan de gezelligheid op de congressen en cursussen die we bezocht hebben, waar we meestal nog een paar daagjes aan vast plakten! Onze stapavond in Berlijn zal ik niet snel vergeten! Net zoals aan het samen trainen én volbrengen van de halve marathon.

Mijn andere kamergenootjes, Joost en Michiel, jullie zorgden voor de nodige mannelijke invloed op onze kamer. Bedankt voor de gezelligheid, de ontelbare koppen koffie die jullie voor mij gehaald hebben en de technische ondersteuning als mijn computer weer eens niet deed wat ik wilde.

Antje, Simon en José, toen ik begon met mijn promotie waren we nog maar met zijn vieren. $\mathrm{k}$ voelde me meteen thuis en ik heb erg met jullie gelachen. Heel veel succes met jullie verdere carrière!

Lieke en Andrea, de grootste sportievelingen van de afdeling, jullie waren super leuke collega's en bedankt voor alle gezellige avondjes die we gehad hebben, mede mogelijk gemaakt door de Jeker en Tuutje. Maike, Ellis en Bart, met jullie heb ik het kortst samen gewerkt, maar we hebben in korte tijd genoeg leuke momenten gehad, bedankt! José Castillo-Ortiz and Fariba, it was really nice to have such nice colleagues from Mexico and Iran!

Alle reumatologen en AIOS, bedankt voor jullie interesse in mijn onderzoek en voor alle patiënten die jullie hebben gemotiveerd om mee te doen aan de onderzoeken. Zonder jullie was het niet mogelijk geweest om binnen drie maanden tijd alle Maastrichtse patiënten voor de ComoSpA studie te includeren. Marloes, ik wil jou in het bijzonder bedanken, het was heel prettig om met je samen te werken aan de review.

Ook een woord van dank aan de secretaresses, Marjan, Peggy en Yvonne. Vooral in de laatste fase, toen ik al in Rotterdam woonde, regelden jullie altijd snel de praktische zaken!

Nieuwe collega's van het Sint Franciscus Gasthuis, het is fijn om ook weer in een nieuwe stad en een nieuw ziekenhuis zulke leuke collega's te hebben! Ook door jullie begin ik me al goed thuis te voelen in Rotterdam. Dr. Dolhain en prof. Hazes, bedankt dat jullie mij de kans hebben geboden om aan de opleiding te beginnen! 
Lieve vrienden en familie, bedankt voor jullie vriendschap. Deci'belles, ook al wonen we helaas niet meer allemaal zo dicht bij elkaar in de buurt, gelukkig lukt het om elkaar te blijven zien. Ingeborg, ondanks dat we uiteindelijk op de donderdag avond samen overbleven toen iedereen weg was uit Maastricht, bleef het natuurlijk de leukste avond van de week na het werk! Je moet maar heel vaak naar Rotterdam komen om dit een beetje te compenseren! Eefje, wat ben je een lief vriendinnetje! Bedankt dat ik altijd mijn hart bij je mocht en mag uitstorten. Bijna hadden Mina en een Indonesische bus ervoor gezorgd dat mijn promotie niet tot een einde had kunnen komen, maar gelukkig is alles op zijn pootjes terecht gekomen. Natascha, Merel, Fleur, Marlijn, Renate, bedankt voor onze vriendschap en alle gezellige zondag-middagborrels, weekendjes weg en vakanties, dat er nog maar heel veel mogen volgen! Fleur en Merel, lieve telgjes, bedankt voor al jullie interesse in mijn onderzoek, jullie steun en alle leuke dingen die we samen ondernemen. Monique en Ilona, ondanks dat ik zo lang aan de andere kant van het land heb gewoond en we elkaar niet zo vaak zagen is onze vriendschap blijven bestaan en daar ben ik heel blij mee!

Jozien, wat is het fijn om zo'n lieve en leuke zus te hebben. Na zoveel jaren is het heel fijn om weer wat dichter bij elkaar te wonen en veel vaker koffietjes te kunnen drinken, samen hard te lopen of gewoon even te kletsen.

Lieve papa en mama, jullie hebben mij altijd gesteund en staan altijd voor ons klaar. Jullie waren altijd geïnteresseerd in mijn onderzoek, al konden jullie volgens mij nooit precies na vertellen waar ik nou precies mee bezig was. Zonder jullie was het wel heel lastig geworden om in een korte tijd én mijn promotie in Maastricht af te ronden én aan een nieuwe baan te beginnen én een verhuizing naar Rotterdam te regelen. Bedankt voor alles wat jullie mij hebben bijgebracht en ik ben er trots op dat jullie mijn ouders zijn. 



\section{CURRICULUM VITAE}

Carmen Stolwijk werd op 14 oktober 1987 geboren in Leiderdorp. Na het behalen van haar diploma aan het Stedelijk Gymnasium Leiden in 2005 studeerde zij geneeskunde aan de universiteit van Maastricht. Tijdens haar opleiding deed zij onder andere een coschap neurologie in Pretoria en een keuze-coschap Interne Geneeskunde in Reykjavik, waar haar interesse voor de reumatologie gewekt werd. In het laatste jaar van haar opleiding volgde ze haar klinische- en wetenschappelijke stage op de afdeling reumatologie van het Maastrichts Universitair Medisch Centrum (MUMC) en in 2011 behaalde ze haar artsendiploma.

In september 2011 begon zij aan haar promotieonderzoek op de afdeling reumatologie van het MUMC onder leiding van prof. A. Boonen en dr. A. van Tubergen. Tijdens haar promotieonderzoek volgde zij verschillende blokken van de master epidemiologie aan de universiteit van Maastricht. De resultaten van haar promotie onderzoek werden gepresenteerd op verschillende nationale en internationale congressen en resulteerde in dit proefschrift.

In december 2014 begon zij met haar opleiding tot reumatoloog in het Erasmus Medisch Centrum te Rotterdam (opleider dr. Dolhain). Op dit moment is zij bezig aan haar vooropleiding interne geneeskunde in het Sint Franciscus Gasthuis te Rotterdam (opleider dr. Rietveld). 



\section{LIST OF PUBLICATIONS}

- Stolwijk C, Boonen A, van Tubergen A, Reveille JD. Epidemiology of spondyloarthritis. Rheum Dis Clin North Am. 2012 Aug;38(3):441-76.

- Stolwijk C, Pierik M, Landewé R, Masclee A, van Tubergen A. Prevalence of spondyloarthritis features in a cohort of patients with inflammatory bowel disease Can J Gasteroenterol. 2013 Apr;27(4):199-205.

- Ramiro S, van Tubergen A, Stolwijk C, Landewe R, van den Bosch F, Dougados M, et al. Scoring radiographic progression in ankylosing spondylitis: Should we use the modified Stoke Ankylosing Spondylitis Spine Score (mSASSS) or the Radiographic Ankylosing Spondylitis Spinal Score (RASSS)? Arthritis Res Ther. 2013 Jan 17;15(1):R14.

- Stolwijk C, van Tubergen A, Ramiro S, Essers I, Blaauw M, van der Heijde D, Landewé R, van den Bosch F, Dougados M, Boonen A. Aspects of validity of the self-administered comorbidity questionnaire (SCQ) in patients with ankylosing spondylitis. Rheumatology (Oxford). 2013;53(6):2054-64

- Ramiro S, Stolwijk C, van Tubergen A, van der Heijde D, Dougados M, van den Bosch F, Landewé R. Evolution of radiographic damage in Ankylosing Spondylitis: a 12-year prospective follow-up of the OASIS study. Ann Rheum Dis. 2015;74:52-9

- Stolwijk C, van Tubergen A, Castillo-Ortiz J, Boonen A. Prevalence of extra-articular manifestations in patients with ankylosing spondylitis: a systematic review and metaanalysis. Rheum Dis. 2013 Sep 2. doi: 10.1136/annrheumdis-2013-203582

- Stolwijk C, Essers I, van Tubergen A, Boonen A, Bazelier M, de Bruin M, de Vries F. The epidemiology of extra-articular manifestations in ankylosing spondylitis: a population-based matched cohort study. Ann Rheum Dis. March 21. doi: 10.1136/ annrheumdis-2014-205253. [Epub ahead of print]

- Ramiro S, van Tubergen A, Stolwijk C, van der Heijde D, Royston P, Landewé R, Reference intervals of spinal mobility measures in normal individuals - the mobility study. Ann Rheum Dis published 24 Mar 2014. Doi: 10.1136/annrheumdis-2013-204953. [Epub ahead of print]

- Ramiro S, van Tubergen A, van der Heijde D, Stolwijk C, Bookelman G, Dougados M, van den Bosch F., Landewé R. Erosions and sclerosions precede the subsequent develop- 
ment of syndesmophytes at the same site: a 12-year prospective follow-up of the OASIS study in patients with ankylosing spondylitis. Arthritis Rheumatol 2014;66:2773-9

- Ramiro S, van der Heijde D, van Tubergen A, Stolwijk C, Dougados M, van den Bosch F., Landewé R. Higher disease activity leads to more structural damage in the spine in ankylosing spondylitis: 12-year longitudinal data from the OASIS cohort. Ann Rheum Dis 2014;73:1455-61

- Stolwijk C, Ramiro S, Vosse D, Landewé R, van der Heijde D, van Tubergen A. Comparison of tests for lumbar flexion and hip function in patients with axial spondyloarthritis and in normal individuals. Arthritis Care \& Res published 3 Sep 2014. Doi: 10.1002/ acr.22464. [Epub ahead of print]

- Ramiro S, van Tubergen A, Stolwijk C, van der Heijde D, Landewé R. Neutral lateral fingertip-to-floor distance can be derived from height. Ann Rheum Dis. 2014; 73:1748-9

- Essers I, Ramiro S, Stolwijk C, Blaauw M, Landewé R, van der Heijde D, Van den Bosch $F$, Dougados M, van Tubergen A. Characteristics associated with the presence and development of extra-articular manifestations in ankylosing spondylitis: 12-year results from OASIS. Rheumatology (Oxford) published 17 Sep 2014. doi: 10.1093/ rheumatology/keu388. [Epub ahead of print]

- Essers I, Stolwijk C, Boonen A, De Bruin ML, Bazelier MT, de Vries F, van Tubergen A. Ankylosing spondylitis and risk of ischaemic heart disease: a population-based cohort study. Ann Rheum Dis. 2014 Oct 31. pii: annrheumdis-2014-206147. doi: 10.1136/annrheumdis-2014-206147. [Epub ahead of print]

- Stolwijk C, Schiepers S, Schoonbrood T, van Tubergen A, van der Heijde D, Landewé $\mathrm{R}$, Boonen A. Infliximab treatment reduces depressive symptoms in patients with ankylosing spondylitis: a subgroup analysis of a randomized, placebo-controlled trial (ASSERT) (submitted)

- Stolwijk C, van Onna M, Boonen A, van Tubergen A. The global prevalence of spondyloarthritis: a systematic review and meta-regression analysis (submitted)

- Stolwijk C, Castillo-Ortiz J, Gignac M, Luime J, Boonen A. Importance of contextual factors when measuring Work Outcome in Ankylosing Spondylitis: a systematic review by the OMERACT Worker Productivity Group (accepted for publication in Arthritis Care \& Res) 
- Ramiro S, Landewé R, van der Heijde D, Stolwijk C, Dougados M, van den Bosch F, van Tubergen A. Hierarchy of spinal mobility measures in ankylosing spondylitis: 12-year data from the OASIS cohort. (Submitted)

- Ramiro S, Landewé R, van Tubergen A, Stolwijk C, Dougados M, van den Bosch F, van der Heijde D. Mechanical stress and smoking may modify the effect of disease activity on radiographic progression in patients with ankylosing spondylitis. (Submitted)

- Essers I, Ramiro S, Stolwijk C, Blaauw M, Landewé R, van der Heijde D, van den Bosch F, Dougados M, van Tubergen A. Do extra-articular manifestations influence outcome in ankylosing spondylitis? 12 year results from OASIS (Submitted)

- Webers C, Essers I, Ramiro S, Stolwijk C, Landewé R, van der Heijde D, van den Bosch F, Dougados M, van Tubergen A. Gender-attributable differences in outcome of ankylosing spondylitis: long-term results from the Outcome in Ankylosing Spondylitis International Study (submitted) 